UNITED STATES DEPAKTMENT OF TIE INTERIOR

GEOLCGICAI SURVEY

ANAITSES OF ROCK AND STREAYI-SEDIMENT SAMPLES FROM SOUTIFRN

BRADFIEID CANAI QUADRANGLE, SCUTHEJSTERN ALASRA

By

R. D. Koch, R. I. Eiliott, H. C. Berg, and J. G. Smith

Open-file report $76-486$

1976

This report is preliminary and has not been edited or reviened for conformity with

Gaological Survey standards 
Analyses of rock and stream-sediment samples from southern Bradfield Canal quadrangle, southeastern Alaska

By

R. D. Koch, R. I. Elilott, H. C. Berg, and J. G. Smith

\section{INTRODUCTION}

This report contains the analytical data and a statistical summary for 328 rock and 150 stream-sediment samples from the southern portion of the Bradfield Canal 1:250,000-scale quadrangle, southeastern Alaska. A companion report (Koch and others, 1976) contains all analytical data for geochemical sampling within the Ketchikan 1:250,000 quadrangle. The data reported here are from samples collected by U.S. Geological Survey geologists and field assistants working on two different USGS projects between 1968 and 1973. They comprise all of the normal rock and stream-sediment geochemical samples collected during USGS geological mapping investigations within the Bradfield Canal quadrangle through 1975. In addition, data for 241 samples collected by U.S. Bureau of Mines engineers Tom L. Pittman and Arthur I. Kimball during the Granite Fiords Wilderness Study have icen included.

Studies in the Ketchikan area

The only comprehensive discussion of the geology of the entire Katchikan area is contained in a report by A. F. Buddington and Theodora Chapin (1929). Buddington (1929) also described the Hyder mining district, located near the Canadian border $120 \mathrm{~km}$ northeast of Ketchikan.

Recent geologic investigations in the Bradfield Canal quadrangle began with the Hyder project, which involved reconnaissance mapping in the Hyder 
area in 1968 (Smith, in press). Systematic geochemical sampling of parts of Bradfield Canal 1:63,360 quadrangles A1, A2, A3 and A4 was conducted in conjunction with reconnaissance geologic mapping of the Granite Fiords wilderness study area in 1972 and 1973 (Berg and others, in press). Most of the data contained herein was obtained during this study. The USGS geochemical data from this study were released in the form of a computer tape (NTIS catalogue no. USGS-GD74-009; publication no. PB-232-049) which can be obtained from the National Technical Information Service, Department of Commerce, Springfield, Va. 22161.

\section{Sampling}

The analytical data for the USGS stream-sediment and rock samples are given in tables 5 and 6 respectively. The data for the U.S. Bureau of Mines samples is given in table 7 . Locations of stream-sediment samples and locations of rock samples (including J.S. Bureau of Mines sample localities) are shown on plate 1 . USGS sample locations on plate 1 are designated by station numbers. Samples reported in data tables 5, 6 and 7 are identified by station number, with letters appended to the station numbers to distinguish different samples from the same station.

\section{U.S. Geclogical Survey samples}

Standard procedures were followed in collecting and preparing samples. Stream-sadiment samples were generally collected from the active stream channel above the highest high tide level. Where this was not possibie, samples were collected from bank or terrace degosits adjacent to the channel. 
The rock samples are primarily grab samples chosen to provide data on background values for a lithologic unit or to investigate a mineralized occurrence or an outcrop that was conspicuously iron-stained or contained visible metallic minerals. In and around the Granite Fiords wilderness study area most rock samples were taken at or as close as possible to preplanned helicopter landing sites spaced approximately 1.5 to $3 \mathrm{~km}$ apart. The majority of samples within this group can be considered to be randomly chosen representatives of the domfnant lithologies near the sample site. Outside Granite Fiords, less systematic geochemical sampling was carried out in conjunction with reconnalssance geologic mapping. For this reason, the sample population is somewhat biased in favor of samples that were somehow abnormal (e.g., Iron-stained or sulfide-bearing). No attempt is made here to distinguish vein, iron-stained, sulfide-bearing or other atypical samples from the majority which were chosen to represent background values.

\section{U.S. Bureau of Mines samples}

The samples collected by the U.S. Bureau of Mines come from known prospects, from mineralized areas identified during the Granite Fiords study, and from sites where USGS geochemical samples yielded anomalous analytical values. The USBM samples were generally different in purpose, material sampled and manner of collection from the USGS rock samples. Samples from veins and mineralized zones were obtained either by cutting channels with a moil or by continuous chip cuts. Broad mineralized zones were sampled by combining unfform chips taken by moil or sample pick at regular intervals, usually 0.3 or $0.6 \mathrm{~m}$ ( 1 to $3 \mathrm{ft}$ ), across a representative section. 
The sites sampled by the U.S. Bureau of Mines are shown on plate $I$ by site-numbers such as BM30, BM31, etc. This numbering sequence starts with BM28 and is a continuation of the numbering used for USBM sampie localities in Open File Report 76-427 (Koch and others, 1976). Table 1 lists the sample numbers for each sampled area along with the site designation assigned in the Granite Fiords report. Detailed maps and descriptions of the U.S. Bureau of Mines sample sites are given in the Granite Fiords report (Berg and others, in press). Because of the differences in method and objective of sampling, the samples collected by the Bureau of Mines are Iisted separately (table 7) and are not considered to be directly comparable to the rock samples collected by the USGS.

\section{Analytical procedures}

Stream-sediment samples were dried, sieved, and a split of the -80 mesh fraction was analyzed. Rock samples were pulverized and a split was analyzed. Samples were analyzed for up to 30 elements by the six-step semi-quantitative spectrographic method, and for gold, copper, lead and zinc by atcmic absorption spectrophotometry. The analyses for mercury were done by a flameless atomic absorption technique where mercury vapor is thermaliy released from a crushed sample (Vaughn and McCarthy, 1964). The elements for which analyses were conducted were different for the Hyder project and the Granite Fiords study. The semi-quantitative spectrographic analyses were performed by J. E. Abrams, K. J. Curry, J. M. Mootooka, J. Reynolds, and D. Siems. The atomic absorption analyses vere done by $R$. $B$. Carten, J. G. Frisken, R. W. Leinz, A. L. Meier, R. L. Miller, D. G. Murrey, M. S. Rickard, A. J. Toevs, R. Vaughn, and W. W. Vaughn.

\section{Geochemical data}

The analytical results Iisted in tables 5, 6 and 7 are reported as values such as $7.0 \mathrm{ppa}, 10.00$ percent, etc., or as qualified values expressed 


\begin{tabular}{|c|c|c|c|}
\hline $\begin{array}{l}\text { USBM } \\
\text { locality } \\
\text { no. }\end{array}$ & $\begin{array}{c}\text { Granite Fiord } \\
\text { site } \\
\text { designation }\end{array}$ & Prospect name & $\begin{array}{c}\text { USBM } \\
\text { sample } \\
\text { numbers }\end{array}$ \\
\hline BM28 & $\mathrm{P}-8$ & Cathedral & $\begin{array}{l}72 \mathrm{~K} 033-72 \mathrm{~K} 035 \\
72 \mathrm{P} 124-72 \mathrm{P} 126\end{array}$ \\
\hline BM29 & $P-4$ & Chickamin & $72 \mathrm{~K} 065$ \\
\hline BM30 & $\mathrm{P}-1$ & Marietta & $73 \times 108-73 \times 109$ \\
\hline BM31 & $\mathrm{P}-2$ & Stampede & $72 \mathrm{~K} 050-72 \mathrm{~K} 054$ \\
\hline BM32 & $P-3$ & Double Anchor & $\begin{array}{l}72 \mathrm{~K} 036-72 \mathrm{~K} 049 \\
72 \mathrm{~K} 055-72 \mathrm{~K} 063 \\
72 \mathrm{P} 127-72 \mathrm{P} 128\end{array}$ \\
\hline BM33 & $P-5$ & Lake & $72 \mathrm{P} 121$ \\
\hline $\mathrm{BM} 34$ & $P-7$ & Blasher & $72 \mathrm{P} 091-72 \mathrm{P} 116$ \\
\hline BM35 & $P-6$ & Lakeside & $72 \mathrm{P} 119-72 \mathrm{P} 120$ \\
\hline BM36 & $P-9$ & Hummel Canyon & $73 \mathrm{P} 080-72 \mathrm{P} 081$ \\
\hline BM37 & $P-10$ & Swenning's Greenpoint & $73 \mathrm{~K} 112-73 \mathrm{~K} 1 \geq 5$ \\
\hline $\mathrm{BM} 38$ & $P-16$ & Galena & $73 P 084-73 P 085$ \\
\hline BM39 & $\mathrm{P}-11$ & Greenpoint Group & $72 \mathrm{P} 160-72 \mathrm{P} 167$ \\
\hline BM40 & $p-12$ & Heckla & $\begin{array}{l}72 \mathrm{P} 122-72 \mathrm{P} 123 \\
72 \mathrm{P} 129-72 \mathrm{P} 159\end{array}$ \\
\hline BM41 & $G-56$ & & $73 P 079$ \\
\hline BM42 & $\mathrm{P}-15$ & Edelweiss & $72 \mathrm{~K} 102-72 \mathrm{~K} 103$ \\
\hline BM43 & $P-14$ & Marmot Group, upper basin & $72 \mathrm{~K} 126-72 \mathrm{~K} 140$ \\
\hline BM44 & $P-13$ & Marmot Group, lower basin & $\begin{array}{l}72 \mathrm{~K} 066-72 \mathrm{~K} 101 \\
72 \mathrm{~K} 104-72 \mathrm{R} 125 \\
72 \mathrm{P} 045-72 \mathrm{P} 046 \\
72 \mathrm{P} 168-72 \mathrm{P} 173 \\
72 \mathrm{P} 179-72 \mathrm{P} 189\end{array}$ \\
\hline BM45 & $M-9$ & & $73 \mathrm{~K} 106-73 \mathrm{~K} 107$ \\
\hline BM46 & $P-17$ & Glacier & $73 P 082-73 P 083$ \\
\hline BM47 & $M-8$ & & $72 \mathrm{P} 175-72 \mathrm{P} 178$ \\
\hline BM48 & $P-17$ & Goat Group & $73 \mathrm{~K} 098-73 \mathrm{~K} 105$ \\
\hline $\mathrm{BM} 49$ & $G-54$ & & $73 P 076-73 P 079$ \\
\hline BM50 & $G-50$ & & $73 P 054-73 P 055$ \\
\hline BM51 & $G-47$ & & $73 P 056$ \\
\hline BM52 & $G-46$ & & $73 \mathrm{~K} 078-73 \mathrm{~K} 079$ \\
\hline BM53 & $G-14$ & & $\begin{array}{l}73 K 080 \\
73 K 085-73 K 087\end{array}$ \\
\hline
\end{tabular}

Table 1.--U.S. Bureau of Mines sample localities in the Bradfield Canal quadrangle. 
as a letter. These letter codes are $\mathrm{N}=$ not detected, $\mathrm{L}=$ less than specified limit of determinability, $G=$ greater than value shown, $B=$ no data, $\mathrm{H}=$ interference. The qualification codes $\mathrm{N}$ and $\mathrm{L}$ are preceded by the value of the lower determination limit applicable to that analysis and $G$ is preceded by the upper Iimit. The term $T$ is equal to trace but does not occur in these data. Note that when the right-most digit(s) of an analytical value is zero it is generally not significant. Because the original computer printout is used in these tables, element symbols are in capital letters; for example, the symbol for iron, $\mathrm{Fe}$, becomes $\mathrm{FE}$, magnesium, $\mathrm{Mg}$, becomes MG, and so on. The prefix S stands for spectrographic analysis, AA for atomic absorption, and INST for instrumental (flameless AA) analysis.

The semi-quantitative spectrographic analyses (also referred to as six-step spectrographic analyses) are reported in percentage (\%) or parts per million (PPM) as the midpoints of gecmetric class intervals. The class interval centers and the associated class interval boundaries are those listed below or scme power of 10 times these.

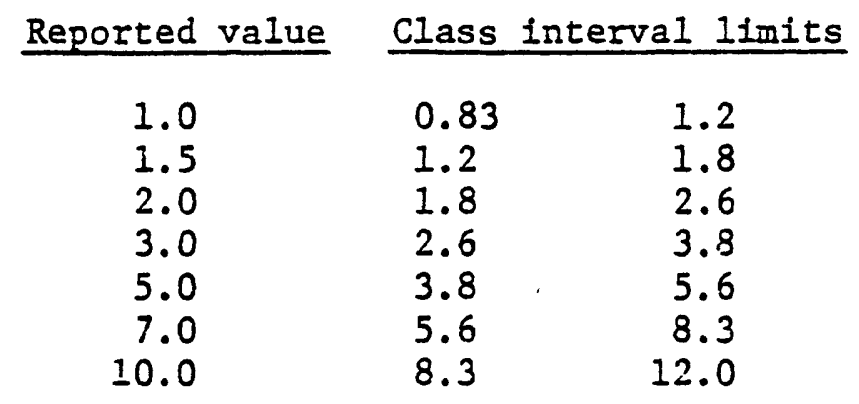

Tests have been performed to determine USGS spectrographic analytical precision (Motooka and Grimes, unpublished data). These tests indicate that the frequency with which values from repeated analyses of the same sample will fall within the class interval containing the "true" value (as measured by the mean of a series of analytical runs) plus or minus one and two adjoining intervals is approximately 83 percent and 96 percent respec- 
tively. For example, if a value is reported as 3.0 the probability is .83 that a repeated analysis would be reported as $2.0,3.0$, or 5.0 . These values are consistent for a variety of geologic materials and show no appreciable difference between elements or concentration ranges (if not near the lower limit of determinability where precision tends to be less). Analyses by the atomic absorption method are not reported on the six-step scale; they are more sensitive and more precise than spectrographic analyses. Minimum limits of determination for each element by spectrographic and atomic absorption analysis are given in table 2.

\section{Statistical summary}

The analytical results from the USGS stream-sediment and rock samples were processed by a computer program known as GEOSUM and are presented in tables 3 and 4 respectively. The GEOSUM program is designed to summarize and tabulate geochemical data--primarily data from semi-quantitative spectrographic analyses. All distributions are treated in terms of the sixstep class intervals described above and thus the atomic absorption data is regrouped to fit into these intervals. The program output consists of: (a) a histogram and freouency distribution table for each element, and (b) a statistical sumary for all elements, which includes geometric means and geometric deviations.

The histograms are on a logarithmic scale and are computed using the same class intervals as those used in the six-step semi-quantitative scale. The histogram bars are composed of X's; each X represents approximately $I$ percent of the total number of samples. Decimal numbers are printed by the computer as powers of 10 , for example: 


\begin{tabular}{|c|c|c|c|c|c|c|}
\hline $\mathrm{S}-\mathrm{Fe}$ & $.05 \%$ & $S-C d$ & 20 & $\mathrm{ppm}$ & $S-S r$ & $100 \mathrm{ppm}$ \\
\hline$S-M g$ & $.02 \%$ & $\mathrm{~S}-\mathrm{Co}$ & 5 & ppm & $S-V$ & $10 \mathrm{ppm}$ \\
\hline $\mathrm{S}-\mathrm{Ca}$ & $.05 \%$ & $\mathrm{~S}-\mathrm{Cr}^{2} /$ & 10 & ppm & $S-W$ & $50 \mathrm{ppm}$ \\
\hline$S-T i 1 /$ & $.002 \%$ & $\mathrm{~S}-\mathrm{Cu}$ & 5 & ppm & $S-Y$ & $10 \mathrm{ppm}$ \\
\hline$S-M n$ & $10 \mathrm{ppm}$ & $\mathrm{S}-\mathrm{La}$ & 20 & ppm & $S-Z n$ & $200 \mathrm{ppm}$ \\
\hline S-Ag & $.5 \mathrm{ppm}$ & S-Mo & 5 & $\mathrm{ppm}$ & $S-Z r$ & $10 \mathrm{ppm}$ \\
\hline S-As & $200 \mathrm{ppm}$ & $\mathrm{S}-\mathrm{Nb}$ & 20 & ppm & $A A-A u^{4 /}$ & $.05 \mathrm{ppm}$ \\
\hline$S-A u$ & $10 \mathrm{ppm}$ & $\mathrm{S}-\mathrm{Ni}$ & 5 & ppm & $\mathrm{AA}-\mathrm{Cu}$ & $5 \mathrm{ppm}$ \\
\hline$S-B$ & $10 \mathrm{ppm}$ & $\mathrm{S}-\mathrm{Pb}$ & 10 & ppm & $\mathrm{AA}-\mathrm{Pb}$ & $5 \mathrm{ppm}$ \\
\hline $\mathrm{S}-\mathrm{Ba}^{2}$ & $20 \mathrm{pps}$ & $S-S b$ & 100 & ppm & $\mathrm{AA}-\mathrm{Zn}$ & 5 ppm \\
\hline $\mathrm{S}-\mathrm{Be}$ & $1 \mathrm{ppm}$ & $S-S c$ & 5 & ppm & Inst- $\mathrm{Hg}$ - $/$ & $.02 \mathrm{ppm}$ \\
\hline$S-B i$ & $10 \mathrm{ppm}$ & $S-S n$ & 10 & ppm & & \\
\hline
\end{tabular}

Table 2.--Lower determination limits for analyses for 1968 through 1973. $S$ - incicates spectrographic analysis, AA - indicates atomic absorption analysis and Inst - indicates flameless AA anaiysis.

$1 / .001 \%$ prior to 1959 .

2/ 5 ppm prior to 1969 .

3/ 5 ppm prior to 1970 .

台 $.02 \mathrm{ppm}$ prior to 1972.

$\underline{5}^{\prime} .01$ ppm prior to 1972 . 


$$
\begin{aligned}
& 7.0 \mathrm{E}-01 \text { means } 7.0 \times 10^{-1} \text { or } 0.7 \\
& 7.0 \mathrm{E} 00 \text { means } 7.0 \times 10^{0} \text { or } 7.0 \\
& 7.0 \mathrm{E} 01 \text { means } 7.0 \times 10^{1} \text { or } 70.0 \\
& 7 . \mathrm{OE} 02 \text { means } 7.0 \times 10^{2} \text { or } 700.0
\end{aligned}
$$

The frequency distribution tables, histograms, and statistics for each element were derived using only data values within the range of anaiytical determination which was valid in 1973. Between 1968 and 1973, the lower limits of determinability for $\mathrm{Au}$ and $\mathrm{Hg}$ analyzed by atomic absofption techniques and for spectrographically analyzed $\mathrm{Ti}, \mathrm{Ba}$, and $\mathrm{Cr}$ were raised. Unqualified values which fell below current determinability limits and values qualified with $\mathrm{N}, \mathrm{L}, \mathrm{G}, \mathrm{T}$, or $\mathrm{H}$ were ignored in these computations. The resulting frequency tables and statistics are biased and the histograms incomplete.

The statistical summaries at the ends of tables 3 and 4 show which elements have qualified values, as well as the number and type of qualification. The summary also recomputes the geometric mean and standard deviation using a method devised by A. J. Cohen for treating censored distributions. If an element has no qualified data values, the mean and standard deviation will be the same in both this statistical sumary and on the page within the table for the particular element. For elements with qualified data, the estimates of mean and standard deviation are unbiased in a strict sense only where the data are derived from a log-normal parent population, but experiments have shown that large departures from this requirerent do not usually invalidate the results. Acceptance and use of the estimates, however, is the responsibility of the user.

The geometric mean is the antilogarithm of the arithmetic mean of the logarithms of the analyses. It is not an estirate of geochemical abundance. 
It is an estimate of "central tendency" (or characteristic value) for a frequency distribution that is approximately symetrical on a logarithmic scale and is useful for characterizing many geochemical distributions. The geometric deviation is the antilogarithm of the standard deviation of the logarithms of the analyses.

For further discussion of geometric mean and standard deviation and of Cohen's method for censored distributions, see Miesch (1963, 1967).

\section{Sampling bias}

In reviewing the sumnary results in tables 3 and 4 , several sources of sampling bias in the data set must be considered. Several factors, including time, weather, snow cover and outcrop exposure prevented uniform sanpling in all areas. Some sites were re-sampled, usually to confirm suspected anomalies or because of other indications of pctential geochemical peculiarity. Sampling density tends to be greater near previously reported prospects or in areas having other indications of mineral enrichment. The uneven coverage and tendency to concentrate sampling in more "interesting" areas have introduced a slight bias into these sumary values. Streamsediment sample density shows less tendency to concentrate near "interestirg" areas but locations of all inland samples are controlled by availability of helicopter landing sites. In addition, it should be kept in mind that tha rock samples have been collected from lithologic units widely separated in location, origin and type. The summary of their values thus provides only a general indication of the trends that may be present.

\section{Acknowledgements}

We wish to thank several USGS colleagues for their considerable cooperation and assistance. George Van Trump, Jr., providsd invaluable aid 
in resolving programing problems. David F. Barnes, Bruce R. Johnson,

Steven K. McDanal, Christine M. McDougal, and Margaret R. Roberts are among those who were especially helpful with various problems encountered during preparation of this report. 


\section{Selected Bibliography}

Berg, H. C. Elliott, R. I., Smith, J. G., Pitman, T. L., and Kimball,

A. I., [in press], Mineral resources of the Granite Fiords wilderness study area, Alaska: U.S. Geol. Survey Bull. 1403.

Buddington, A. F., 1929, Geology of Hyder and vicinity, southeastern Alaska: U.S. Geol. Survey Bull. 807, 124 p.

Buddington, A. F., and Chapin, T., 1929, Geology and mineral deposits of southeastern Alaska: U.S. Geol. Survey Bull. 800, 398 p.

Koch, R. D., Elliott, R. L., Berg, H. C., and Smith, J. G., 1976, Analyses of rock and stream-sediment samples from the Ketchikan quadrangle, southeastern Alaska: U.S. Geol. Survey open-file report 76-427, 256 p. Miesch, A. T., 1963, Distribution of elements in Colorado Plateau uranium deposits--A preliminary report: U.S. Geol. Survey Bull. 1147-E, 57 p. 1967, Methods of computation for estimating geochemical abundance:

U.S. Geol. Survey Prof. Paper 574-B, 15 p.

Smith, J. G. [in press], Geology of the Ketchikan D-I and Bradfield Canal A-I quadrangles, Alaska: U.S. Geol. Survey Bu11. 1425.

Vaughn, W. W., and MCCarthy, J.H., 1964, An instrumental technique for tine determination of submicrogram concentrations of mercury in soil, rock and gas: U.S. Geol. Survey Prof. Paper 501-D, p. D123-D127. 
实哭

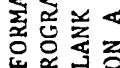

ن

넝

运文尌

\弪

으룰출

응

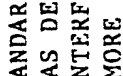

它的

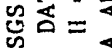

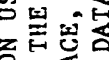

돈

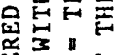

究品是

我记

的悉

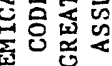

空的

咨运

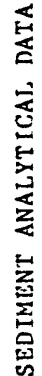

w家

武率界

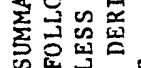

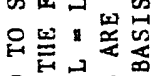

贯. 语总

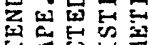

和点密

列起的

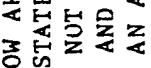

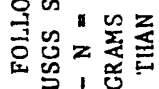

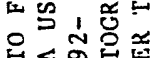

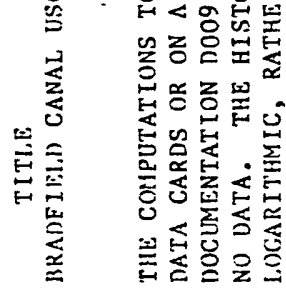

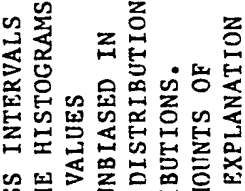

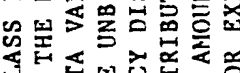

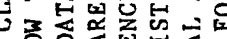

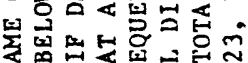

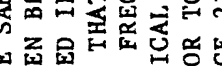

畐

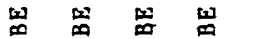

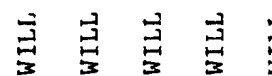

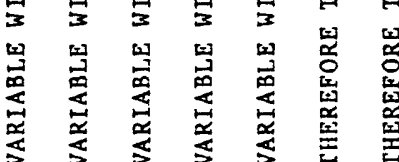

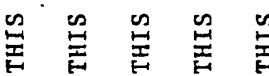

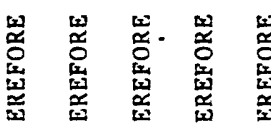

这

些 些

党密

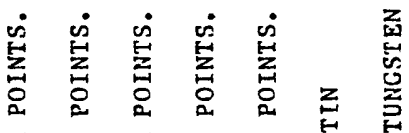

ङ

芒

吉壳志妾录

융ㅇㄹ일

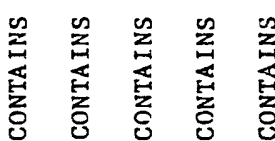

ن

茫

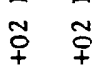

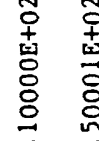

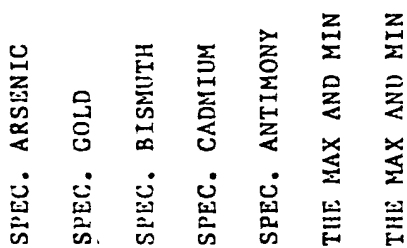
勿

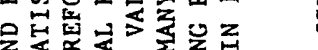

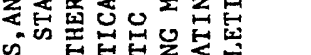

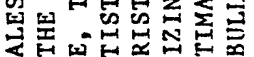

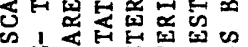

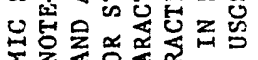

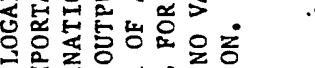

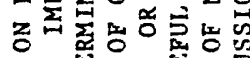

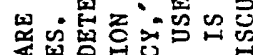

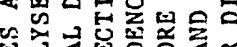

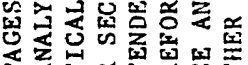

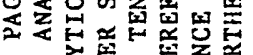

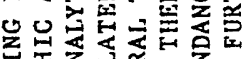

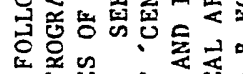

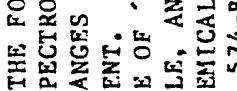

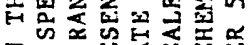
る

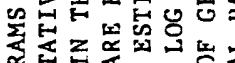

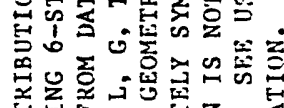

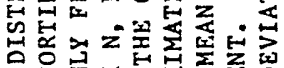

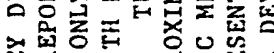

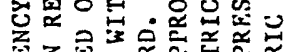

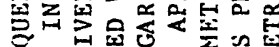

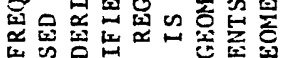

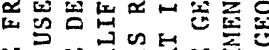

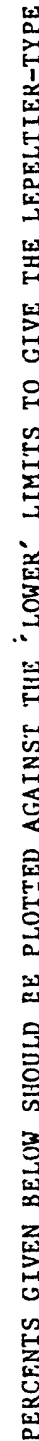

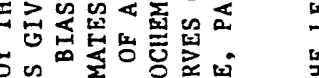

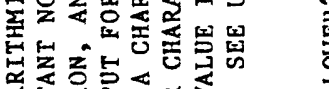

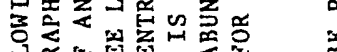

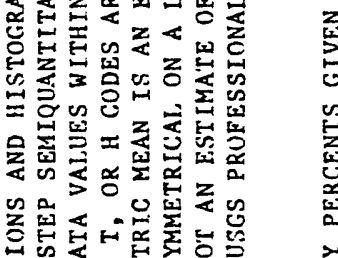




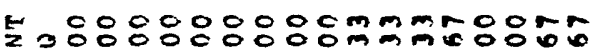
b.

U⿺辶00000000 范占

$00 \stackrel{\circ}{0}$<smiles></smiles>

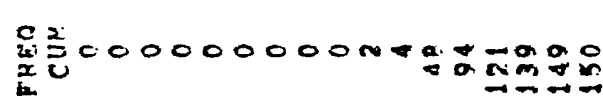

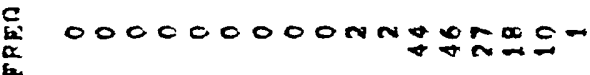

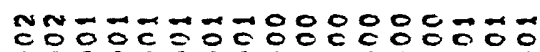
I:인?

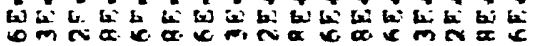

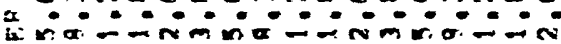
$\stackrel{\substack{a \\ a}}{=}$
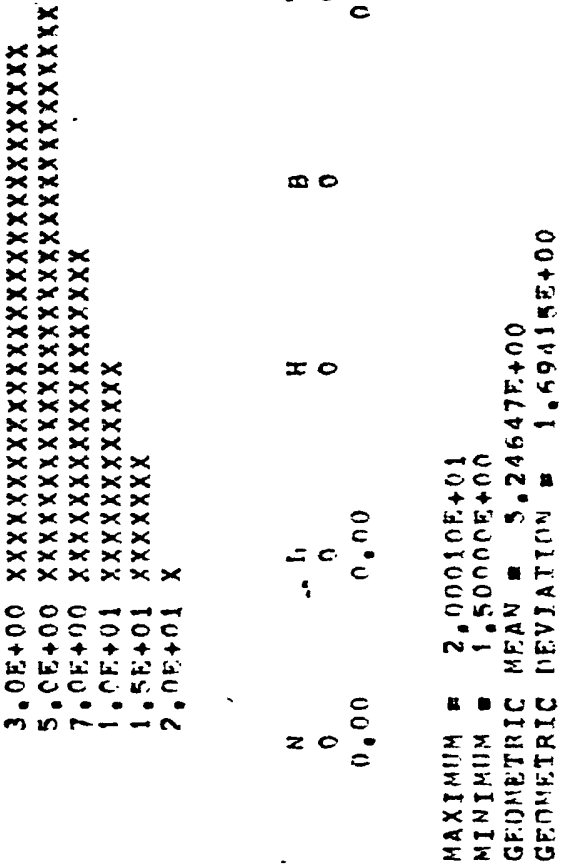


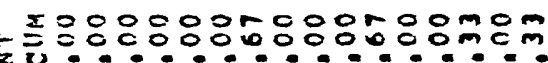
z

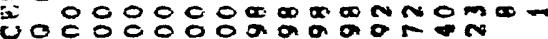
x a文

₹

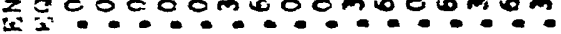
$x<c 000-000$ inomon 西 in $\lim _{\rightarrow} \rightarrow$

$00:$

$-0 \stackrel{8}{\circ}$

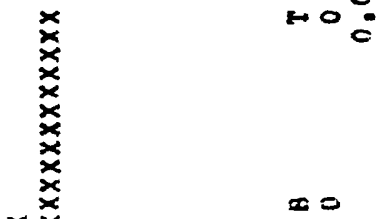

舟竞 $\boldsymbol{s}$

in $00000 n-000 m a n m e n$

$\infty=$

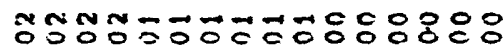

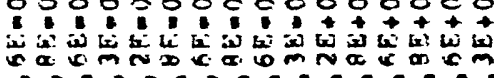

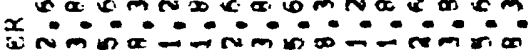

突

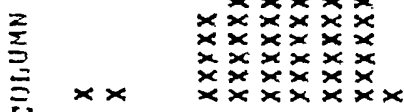

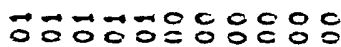

$\Xi \ldots \ldots \ldots \ldots, \ldots$

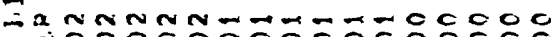

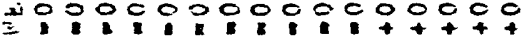

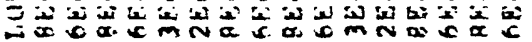

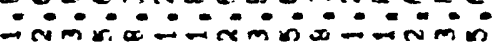
1

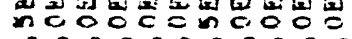

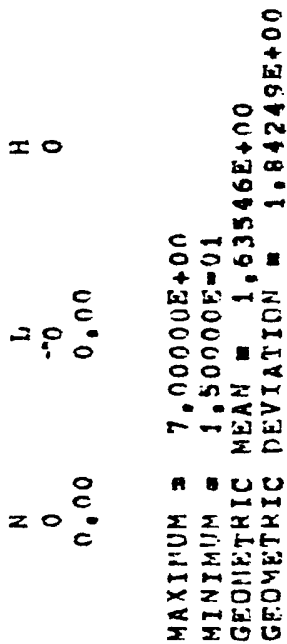




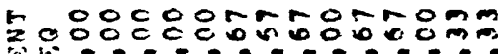

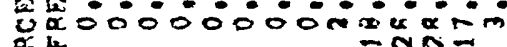

安

这全=m

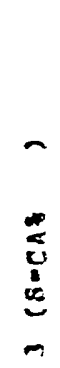

-

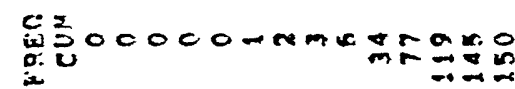

$\rightarrow$

촗

宏文

$00:$

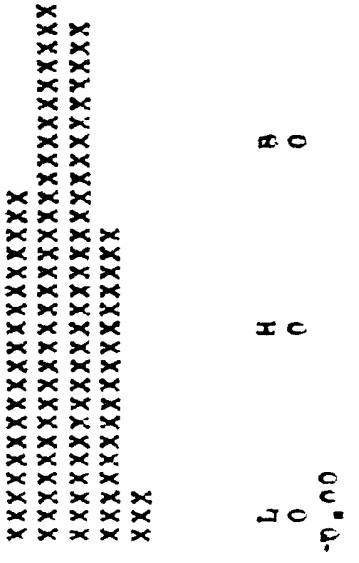

幽畜

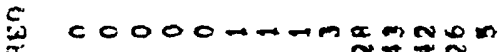

in

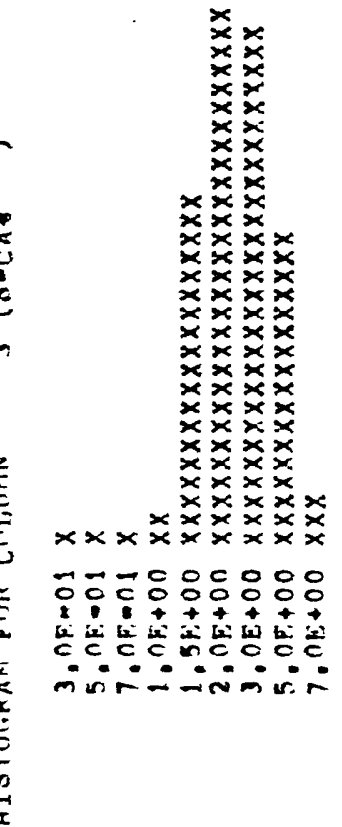

- 0

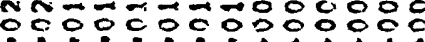

\&

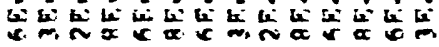

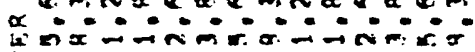
$\frac{5}{2}$

$E$

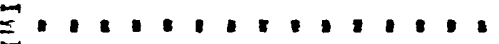

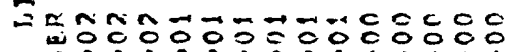

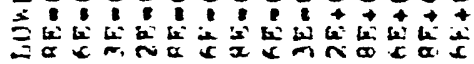

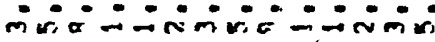




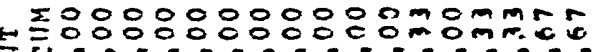
vo:

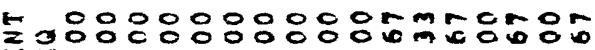

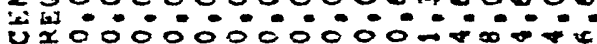

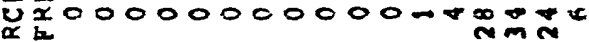
in

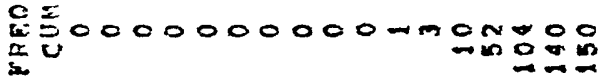
$\checkmark$

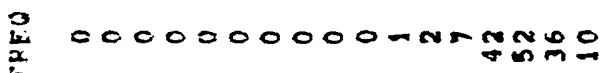

xo

1.5.19.1.1.8.

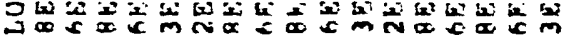

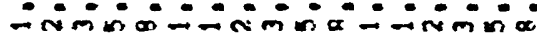
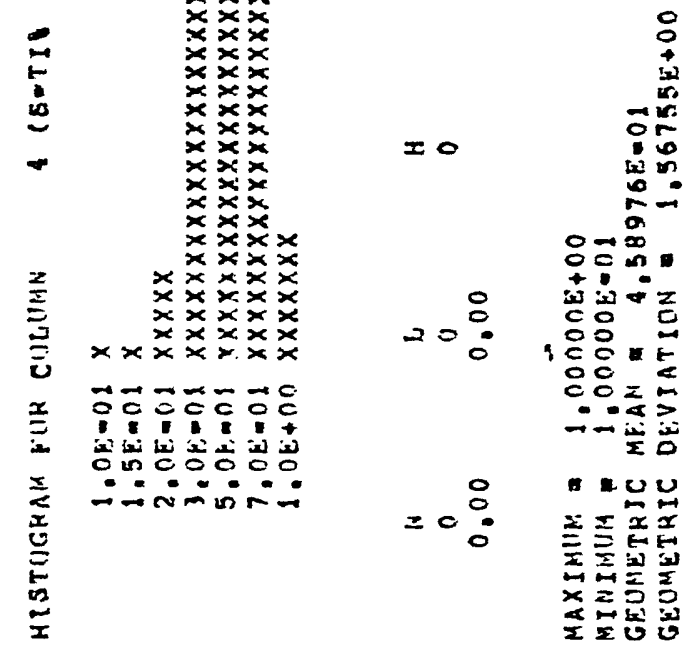


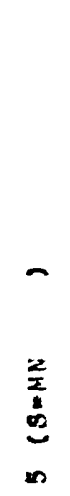

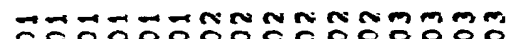

$+t+4+4+4+4+4+4+4$

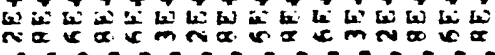

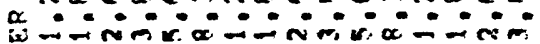
紊

- $\quad=\quad \times x \times x \times x$

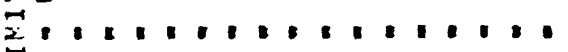

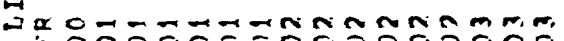

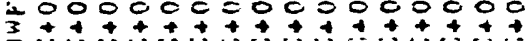

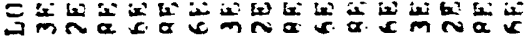

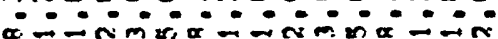

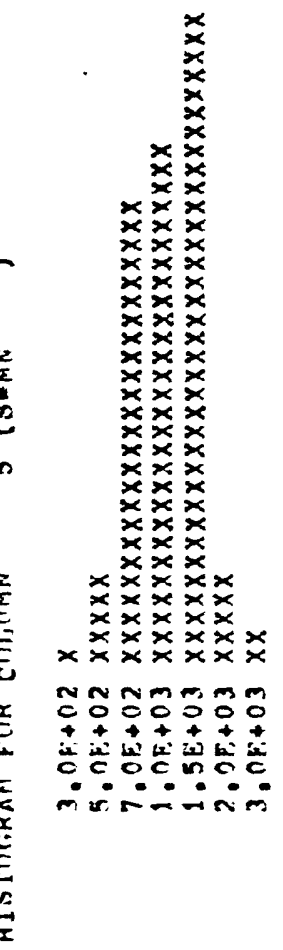

$+0 \stackrel{\circ}{\circ}$

$\infty 0$

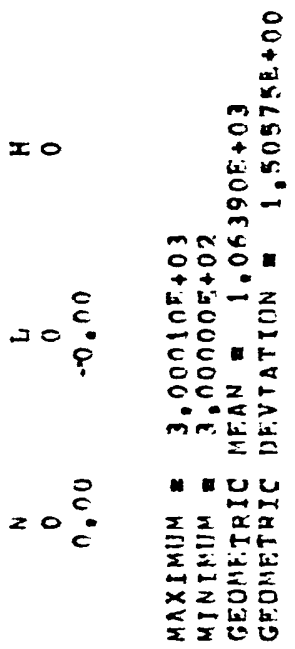




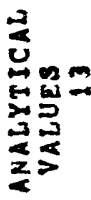

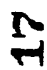
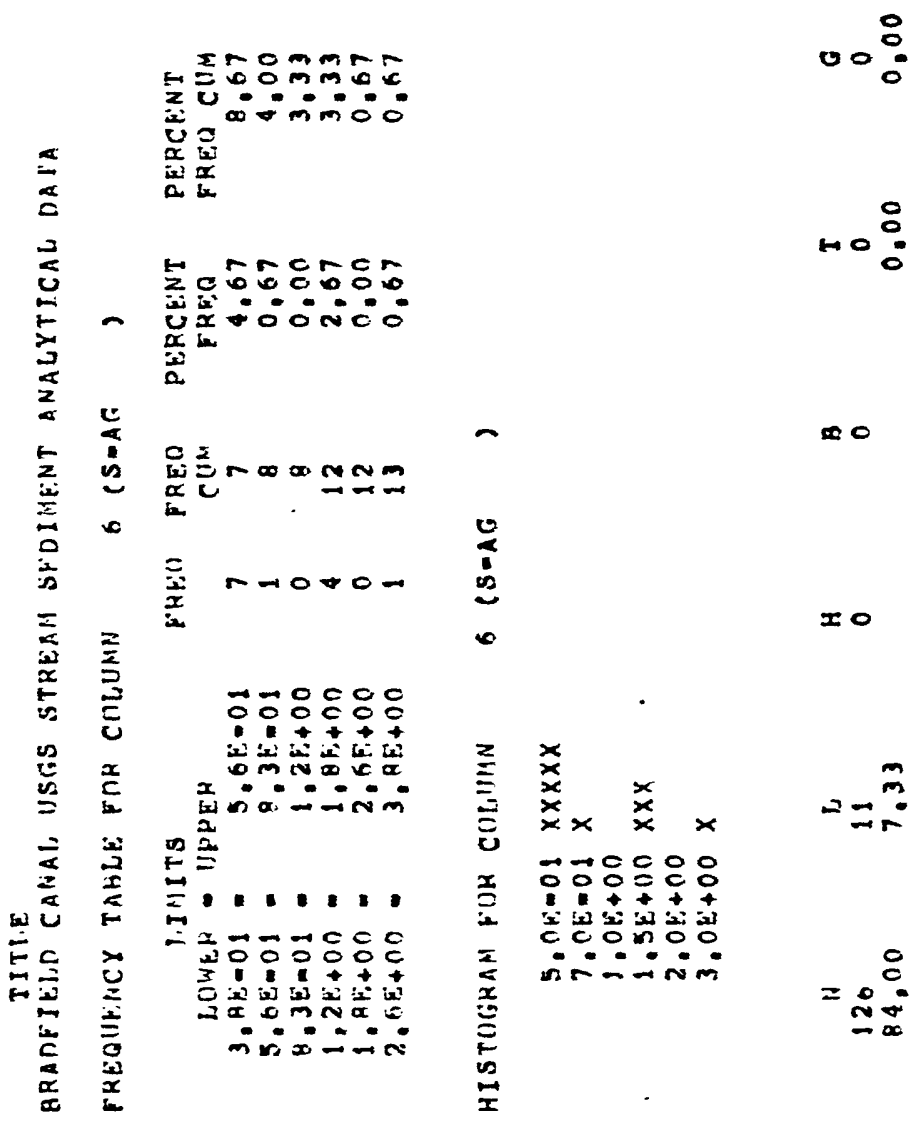

$\infty$

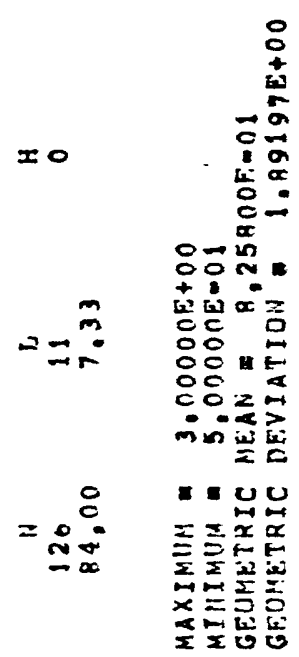



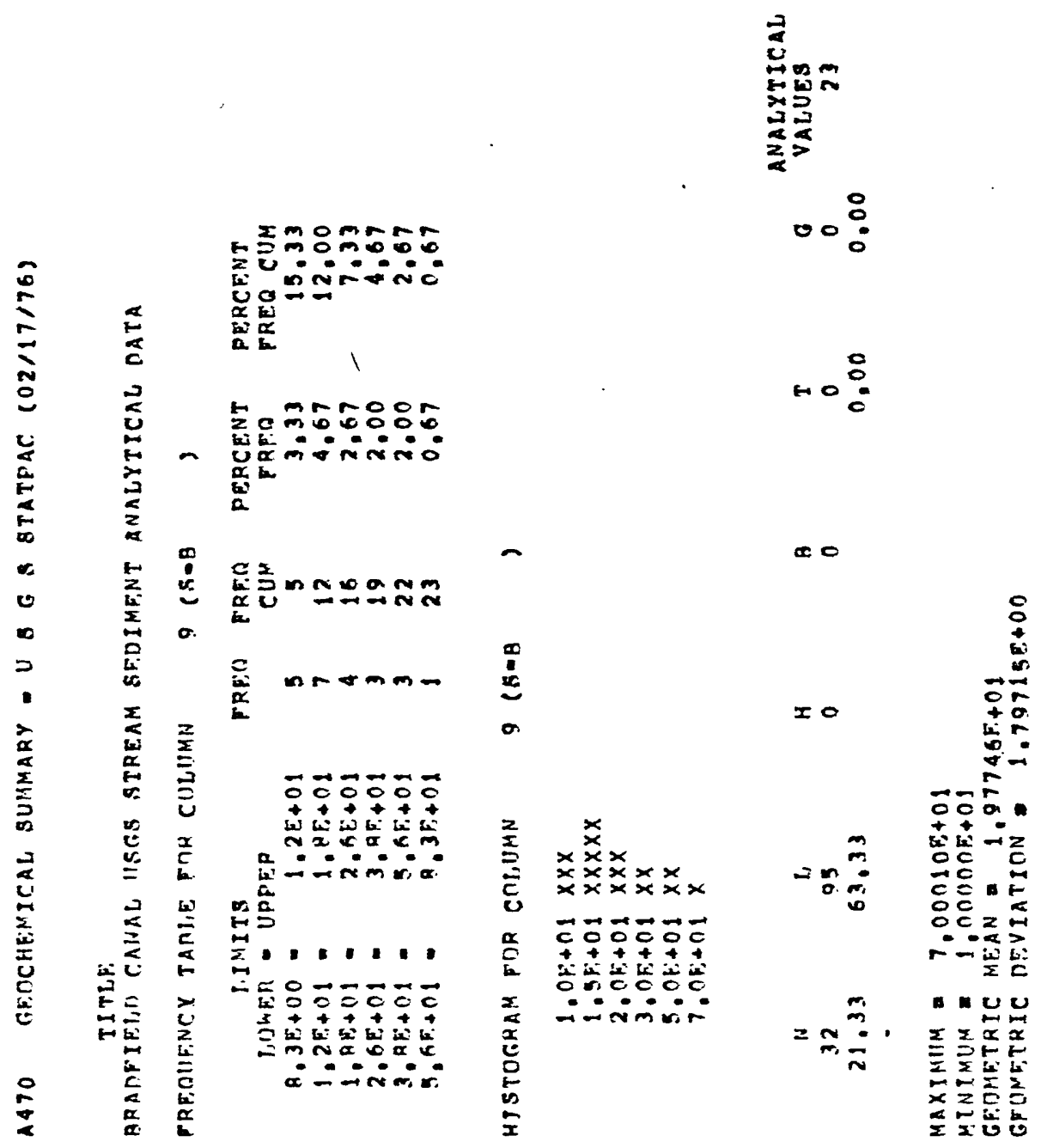


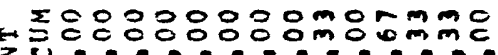

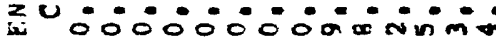

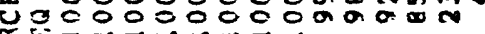

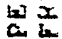

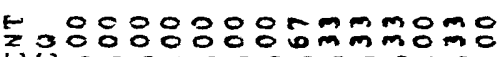

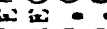

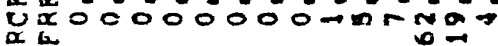
高新

క̛̃

依

$$
\begin{array}{r}
00:- \\
\text { mo: }
\end{array}
$$

$\infty 0$

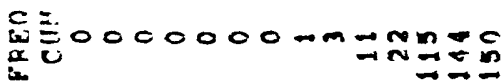

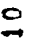

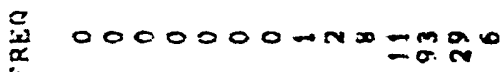

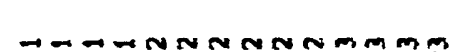

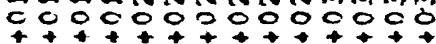

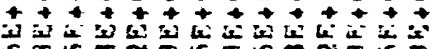

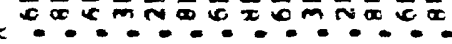

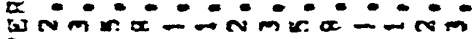
$=\frac{2}{a}$

ว

st.

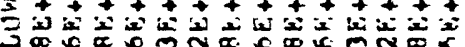

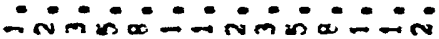

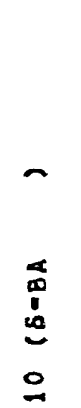

은

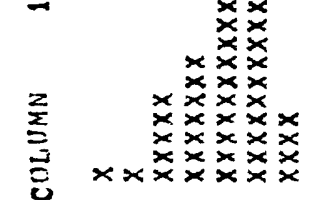

andmmm

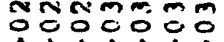

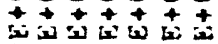

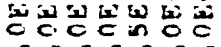

تrir-icim 


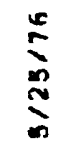

$\underset{c}{a}$

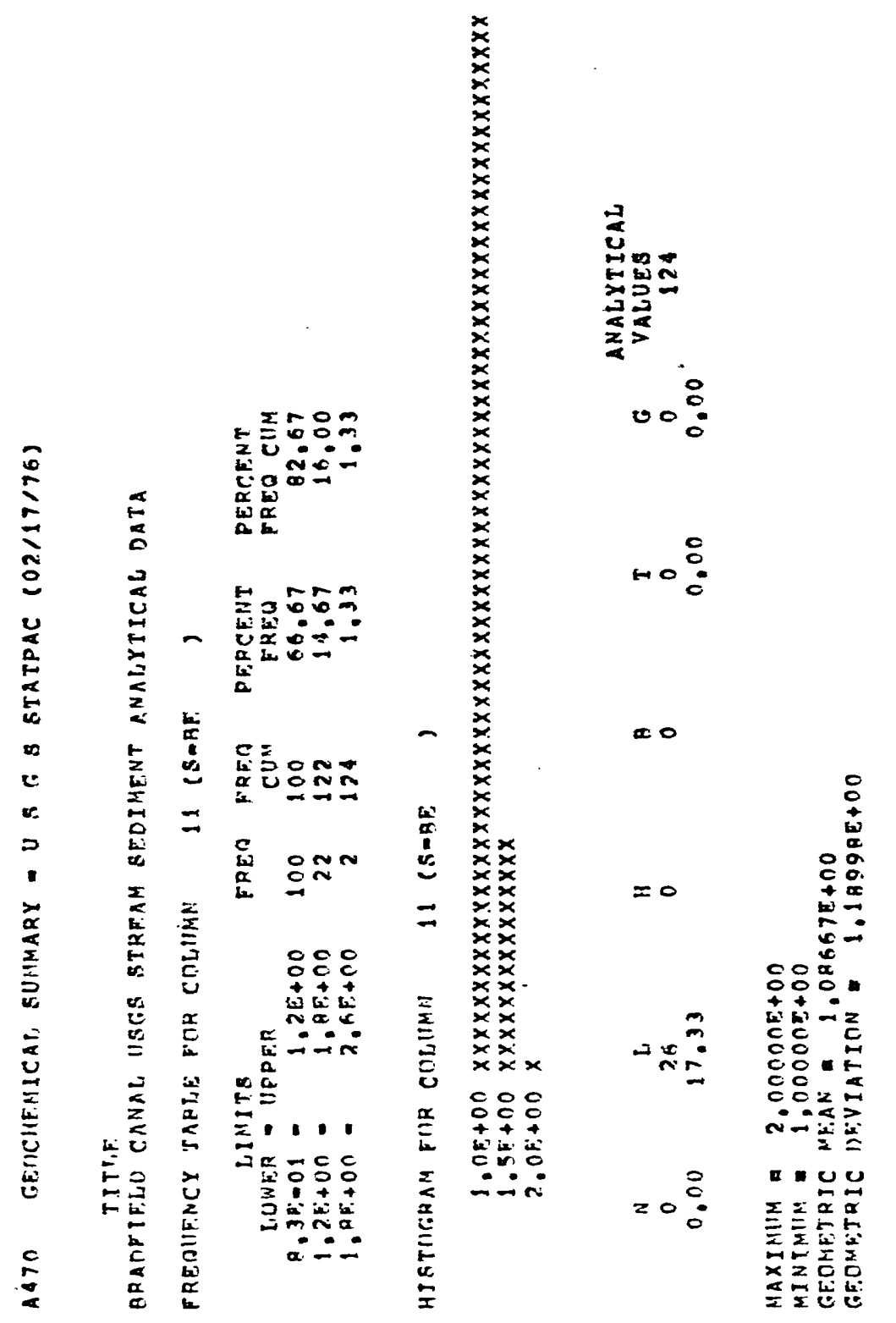




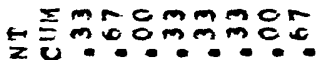
रो

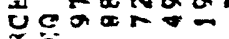

罂

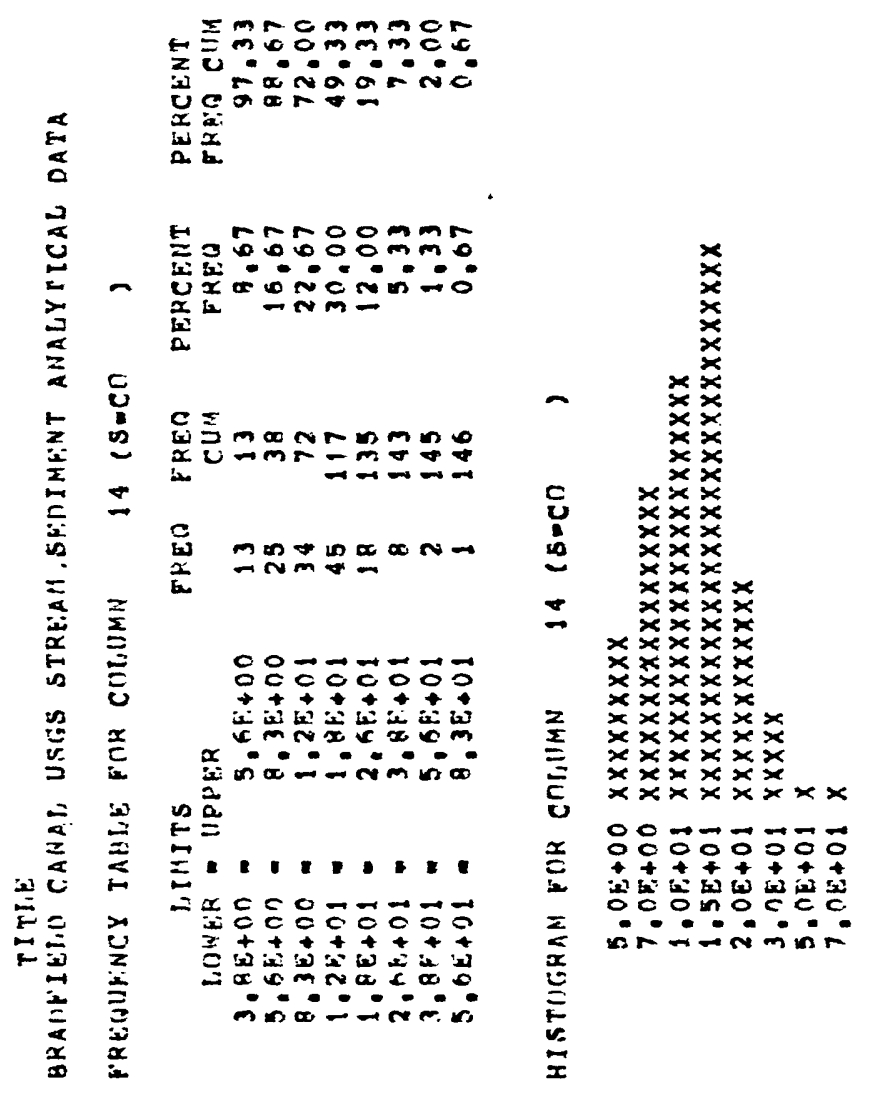

- $0:$

$\infty 0$

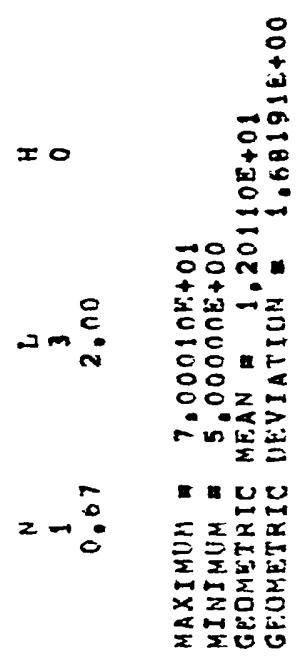


$\sum_{n}^{\infty}$

$\frac{5}{5}$

$-$

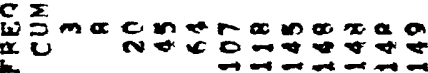

m mangmanmoon

I

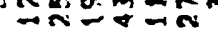

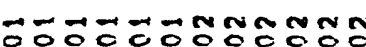

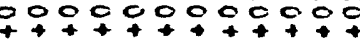

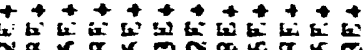

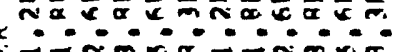

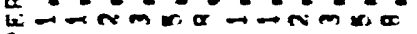

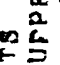

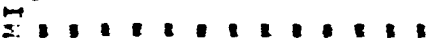

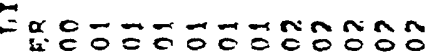

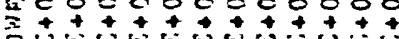

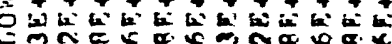

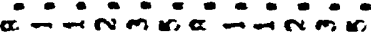

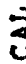

$\infty c$

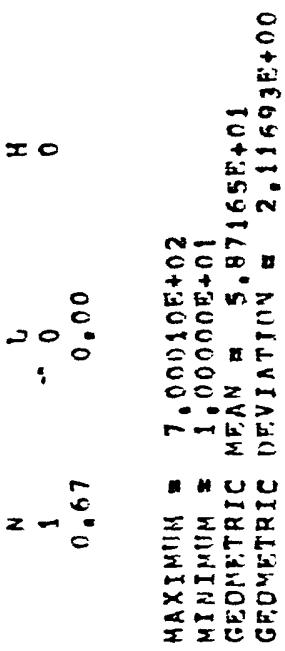




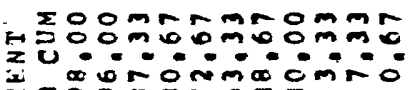

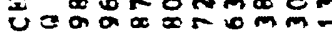

$\infty 0$

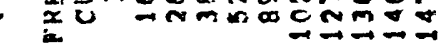

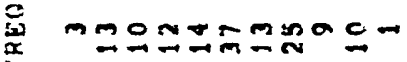
产
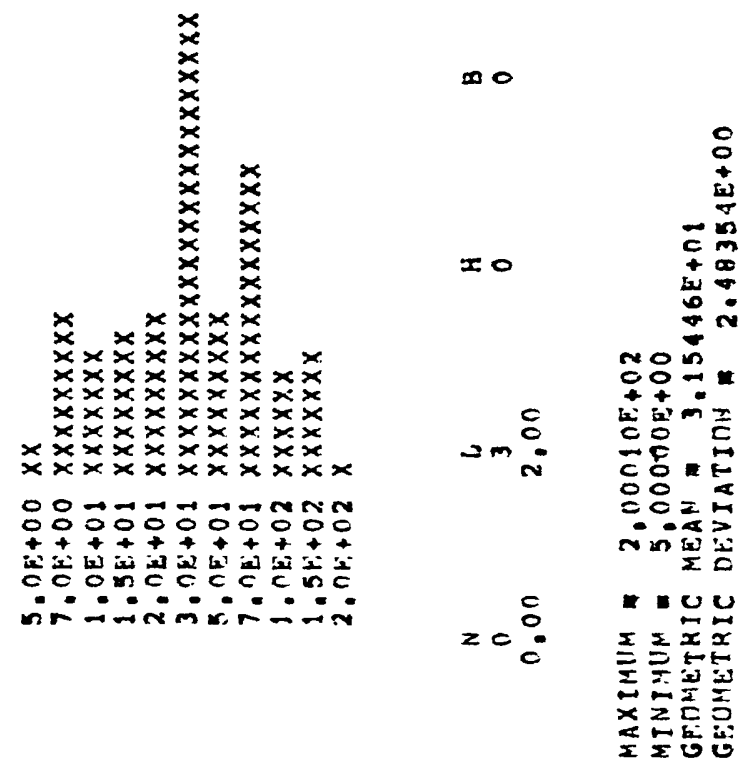
$\stackrel{2}{2}$

$\stackrel{0}{a}$

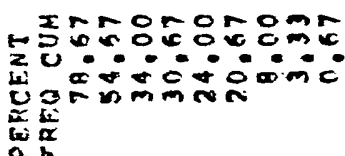

$00 \%$

to

Lc85 050 matas

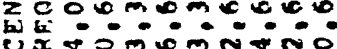

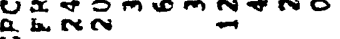

究
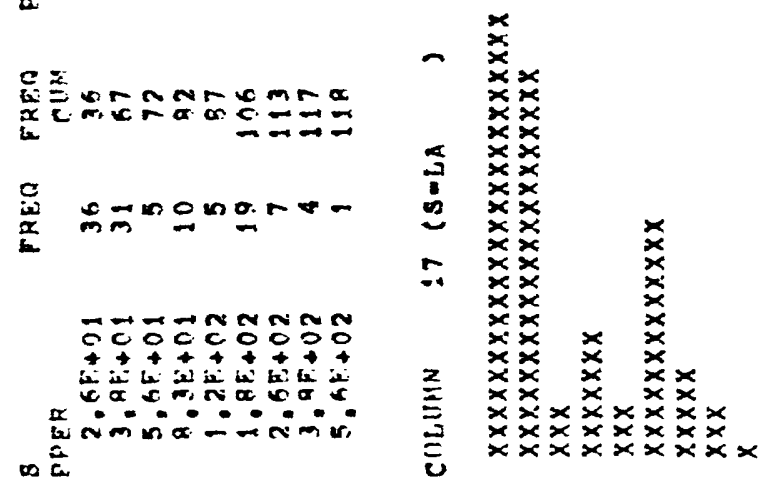

$=0$

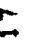

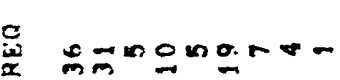

mathnn

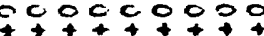

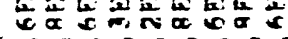

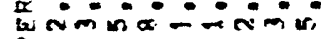

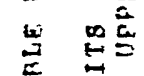

$\equiv .1 .1 .1 .1$

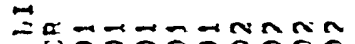

wo $3000000=$

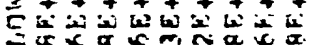

$\because \therefore i n a-i n$

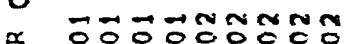

x

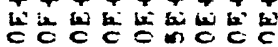

no:

ลิ
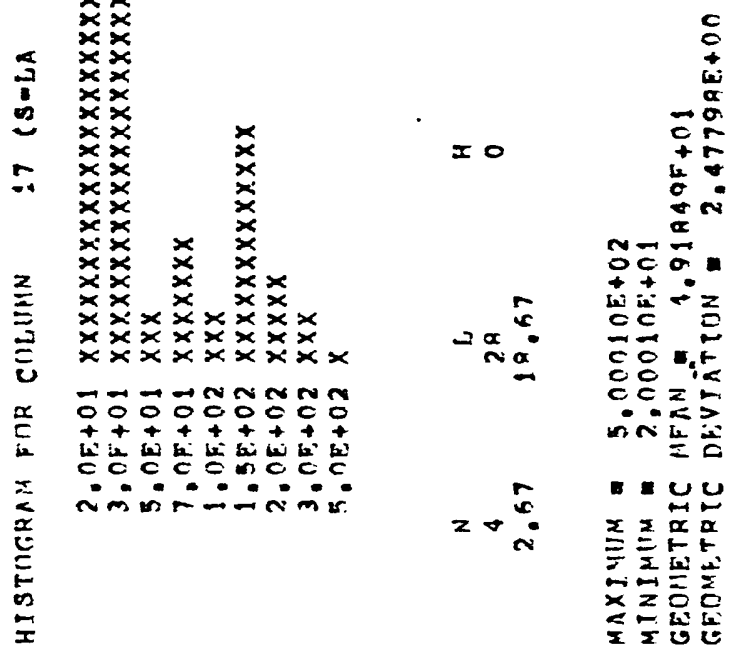
돈드응

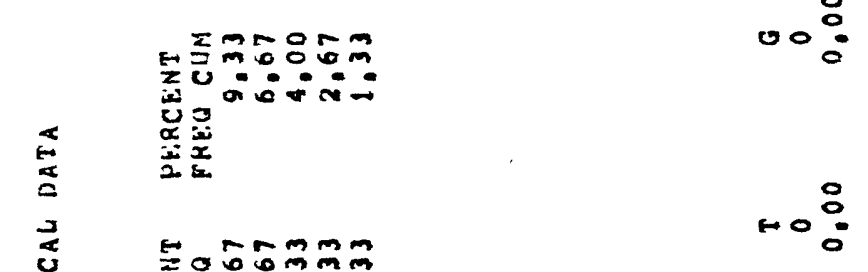

ณ

-

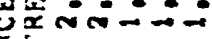
紊

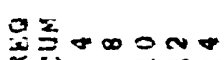

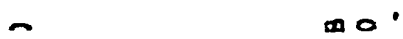

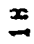

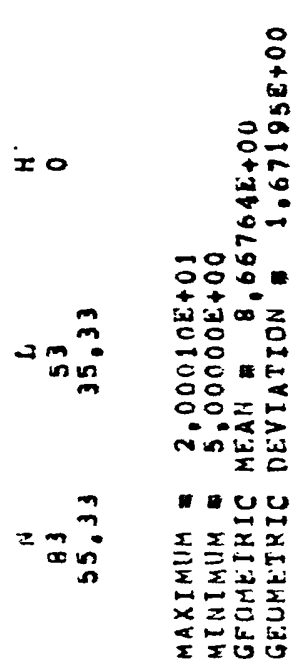




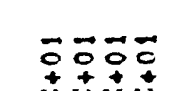

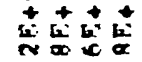
a

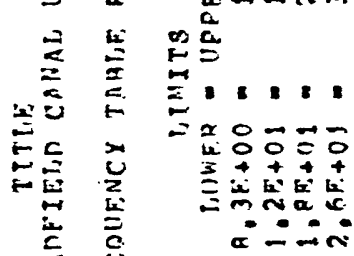

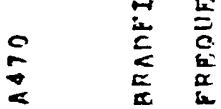

a

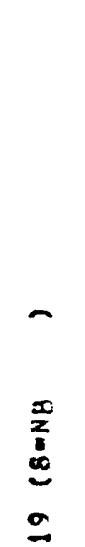

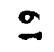

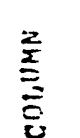

$x \times x$

$\cong \vec{c} \overrightarrow{0} \overrightarrow{0} \overrightarrow{0}$

竞范落药

这

烝

- 0

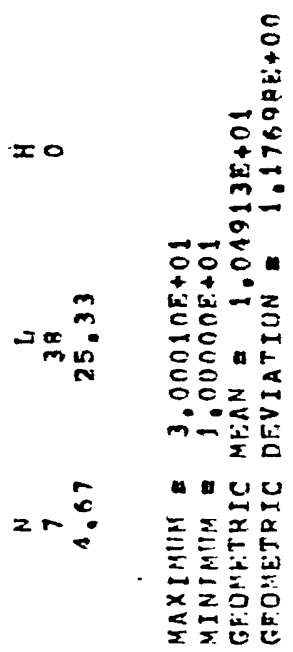




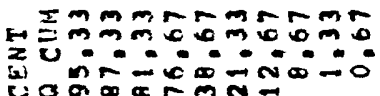

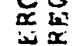

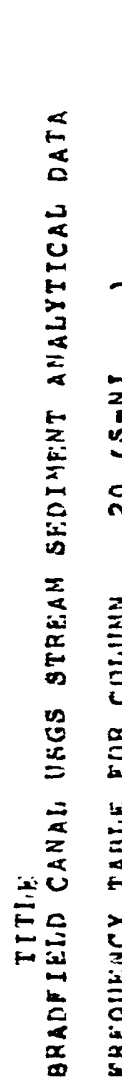

a

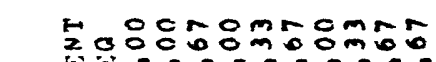

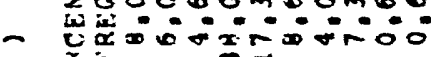
x $m=$$$
2
$$

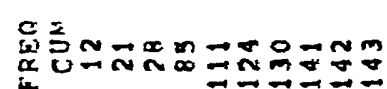

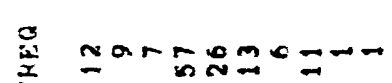

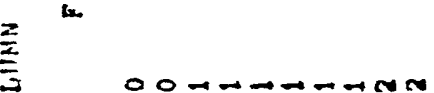

유무으무유

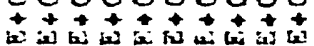

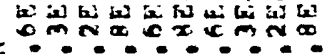

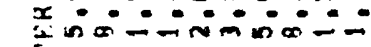
in $\frac{5}{a}$ $\stackrel{2}{a}$

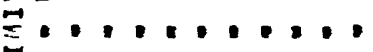

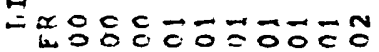

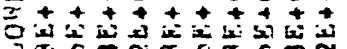

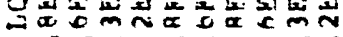

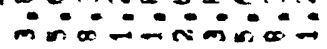

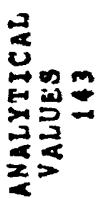

ลิ

$\rightarrow \stackrel{5}{0}$

mo

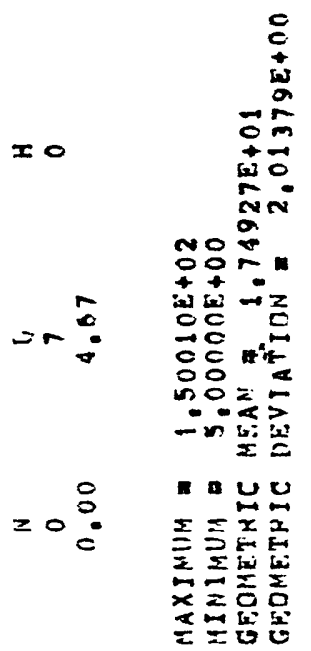




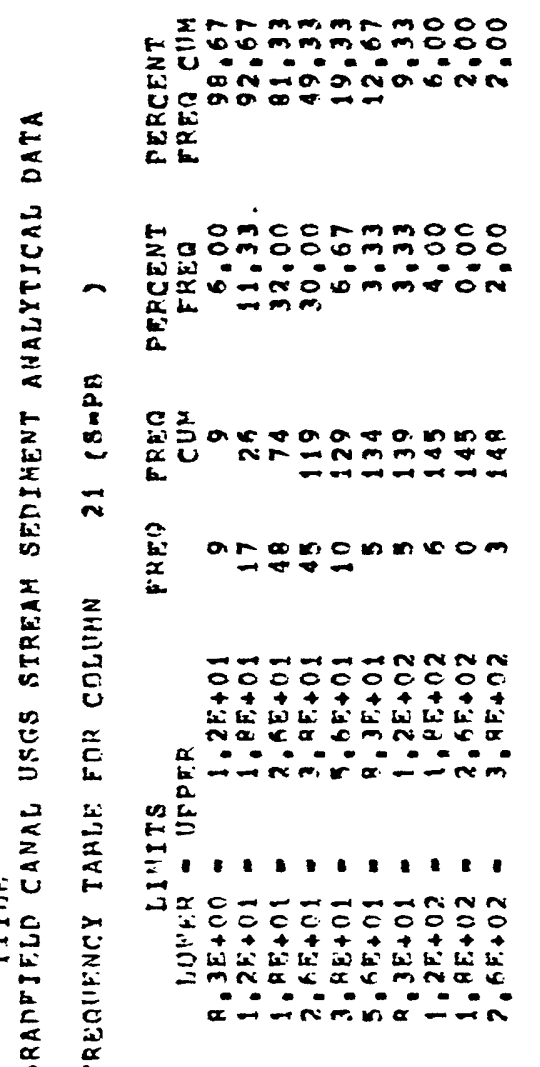

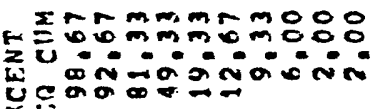

$00 \stackrel{\circ}{\circ}$

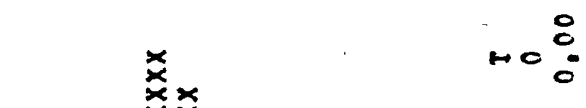

$\infty$

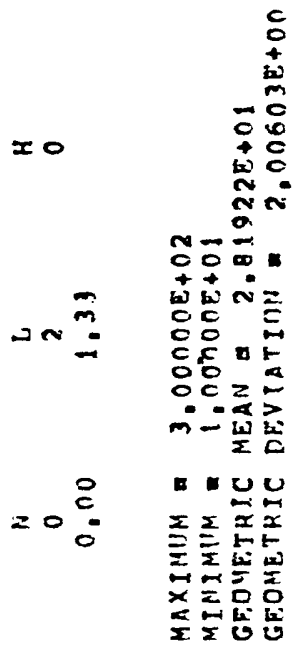



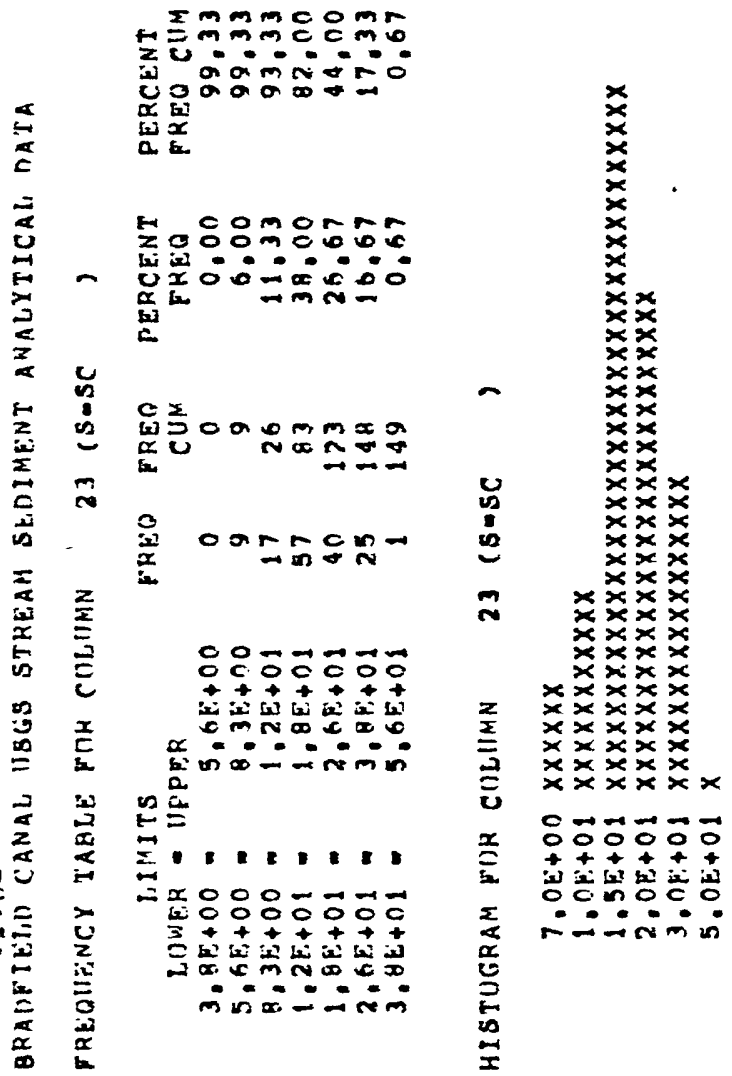

$$
\text { -0: }
$$

Do

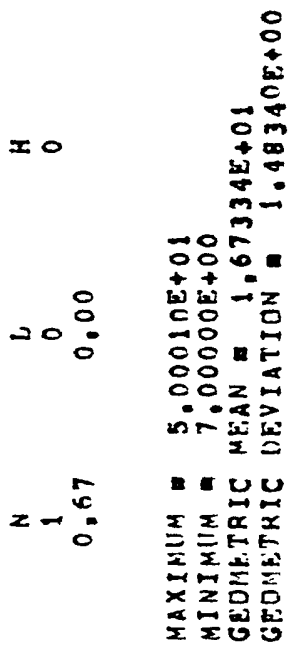



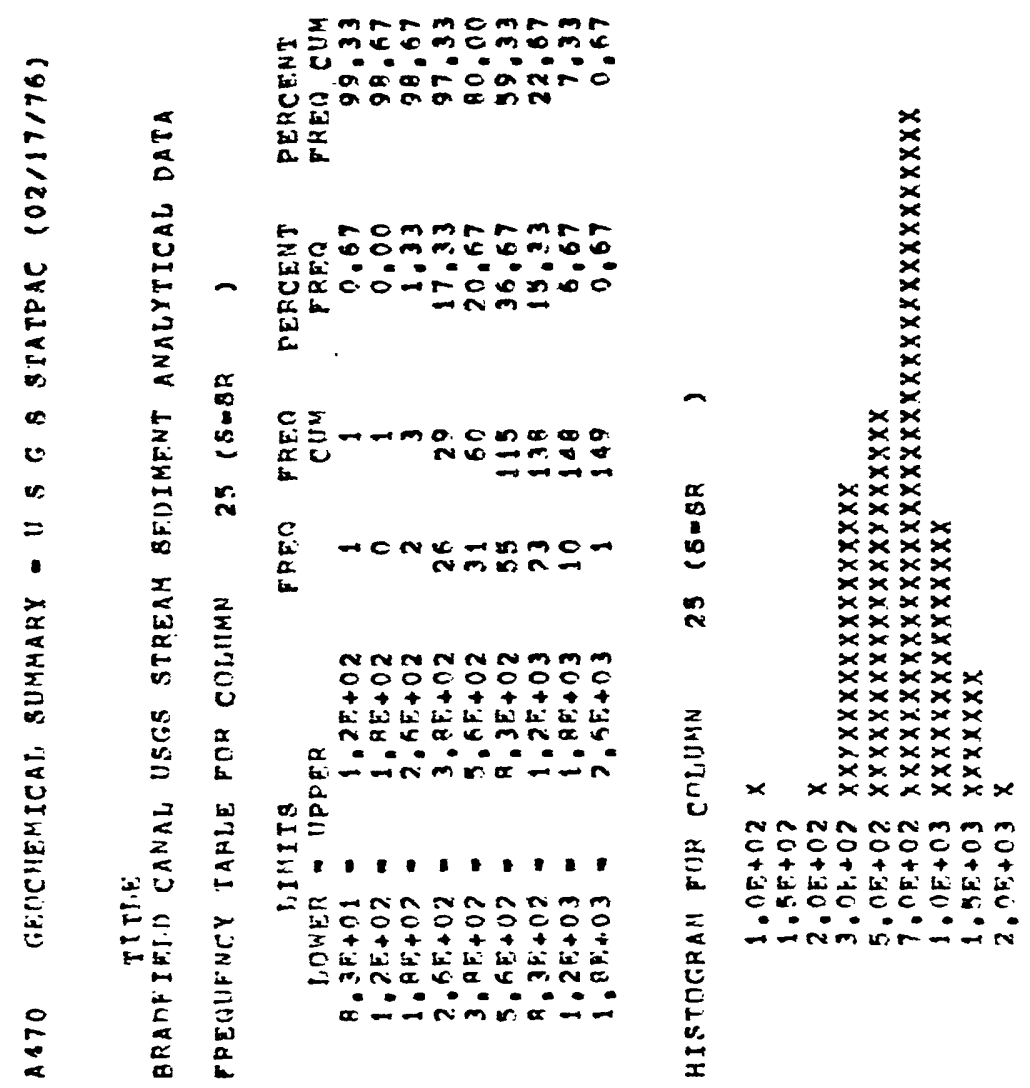

$$
\text { - } 0
$$

II:

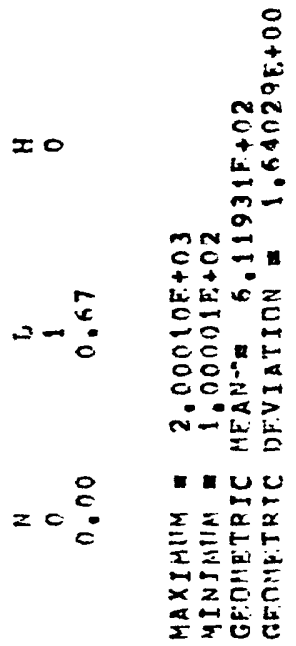




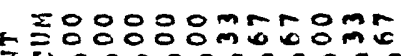

z

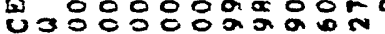

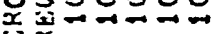

$\stackrel{2}{0}$

$\rightarrow \infty 0$

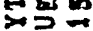

资

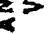

$00 \stackrel{\circ}{0}$

$\rightarrow 0_{0}^{\circ}$

=

is

ux:0000000

更

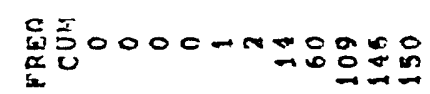

$\stackrel{c}{\sim}$

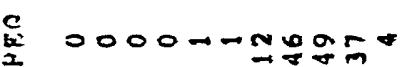

inting

Hame

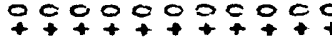

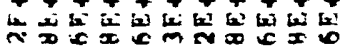

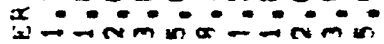

$\stackrel{5=0}{=}$

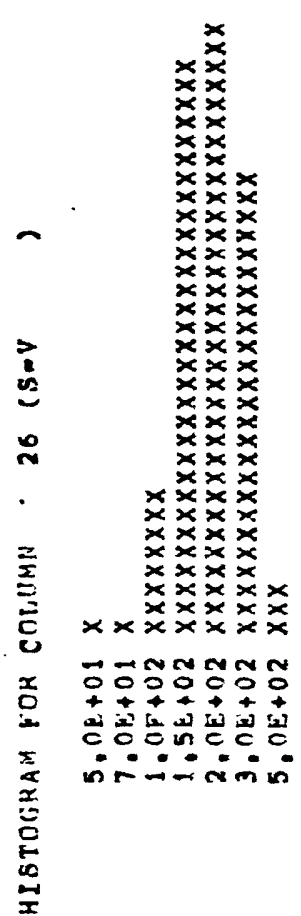

$\infty 0$

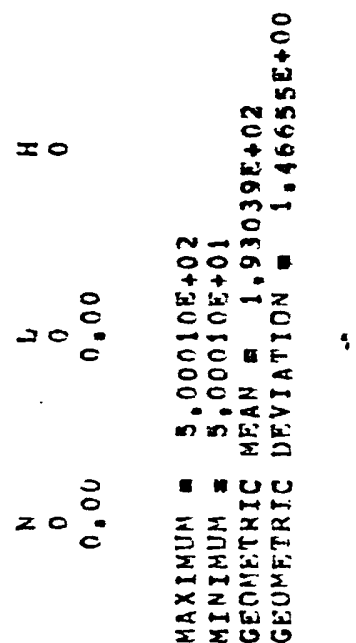


$\sum_{n}^{n}$

$\underline{a}$
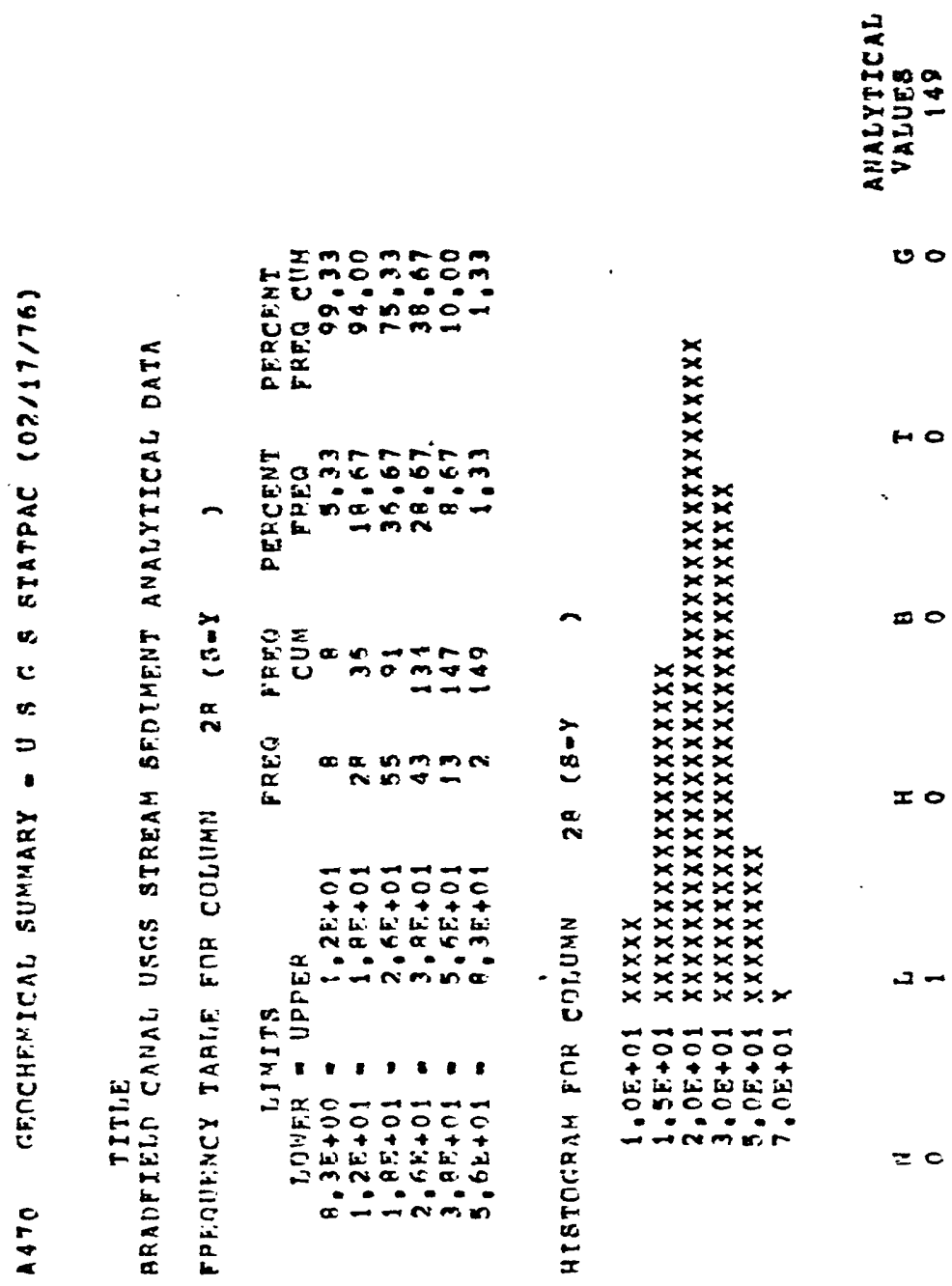

$$
00 \%
$$$$
\text { -O }
$$

$\infty 0$

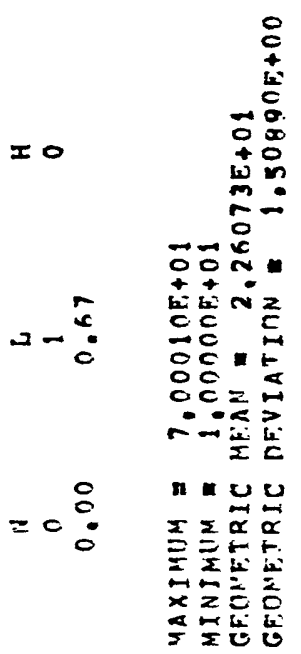




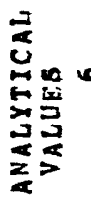

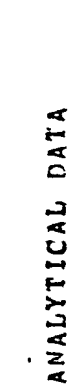

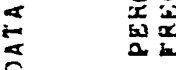

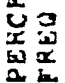

mammico

式岕:

谷

a

< 2

$\sum_{\sin }=\infty \pi m$

xุ๊

s $n-\infty$

$z^{2}=$

$z$
0
0
0
0

$+0 \stackrel{0}{0}$

$\infty$

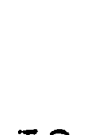

I

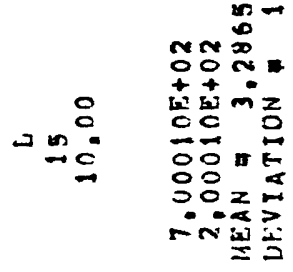

$=\underset{0}{0} \stackrel{0}{0}$

- 40

$\sum \underline{x} \underline{x}$

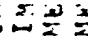

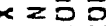

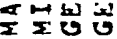


zoㅇㅇㅇㅇㅇㅇㅇㅇㅇ으을

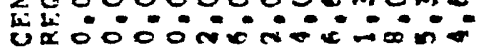

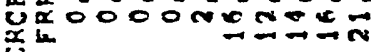
a.

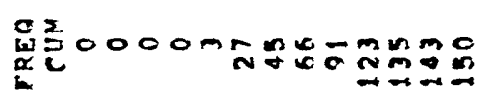

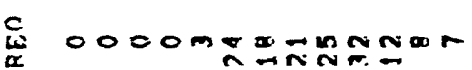
a

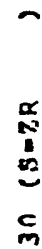

$\rightarrow 0 \stackrel{0}{c}$

$\infty 0$

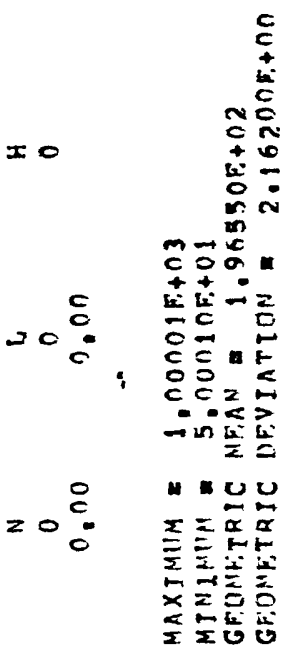




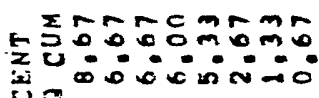

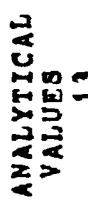

政

a.

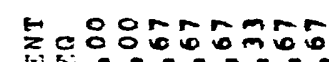

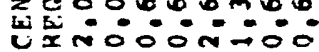

站

OE $m m a n$

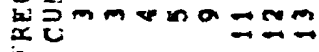
i.

初 mo-m+ns

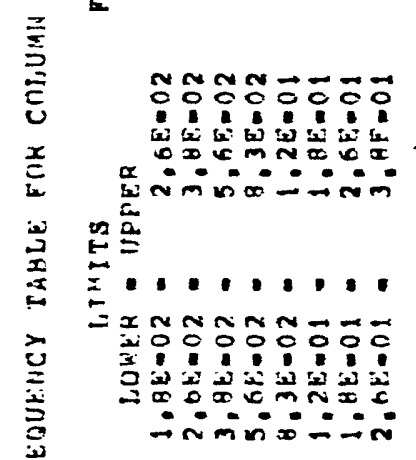

2
$\vdots$
$\vdots$
$\vdots$
$\vdots$
$\vdots$
0

-0:

$00 \div$

$\infty 0$

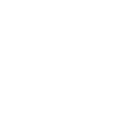

I 0
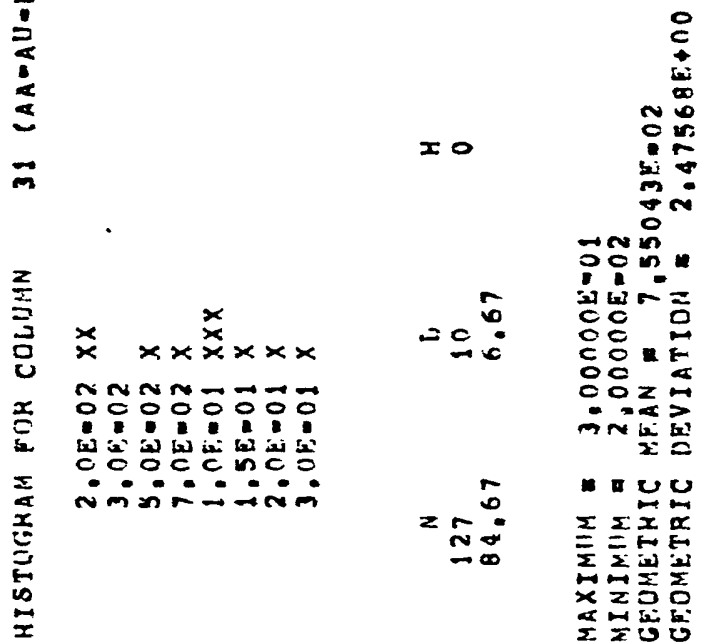

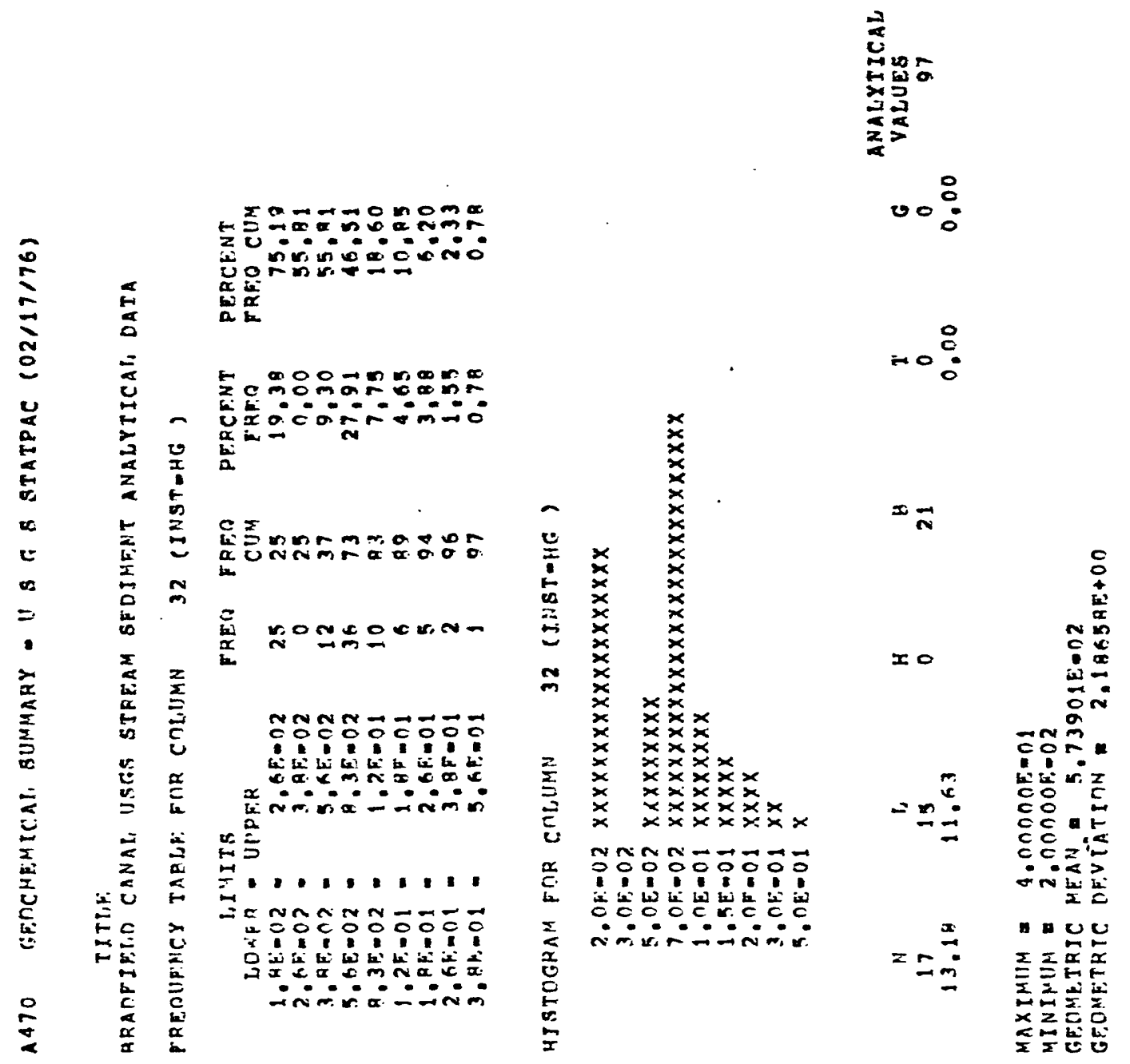


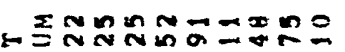

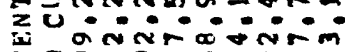

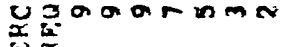

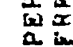

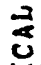

-

촌

$\sum$

$00:$

- : :

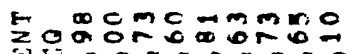

in

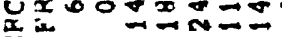

究

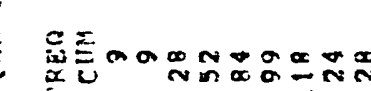

m

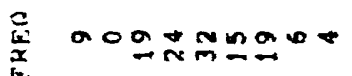

b.

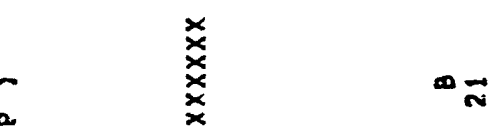

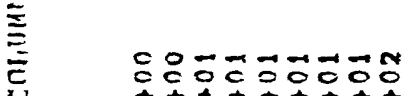

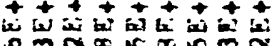

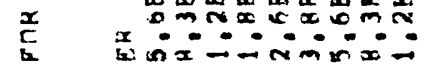

i $\cos ^{2}$

$\stackrel{2}{=}$

…"..."

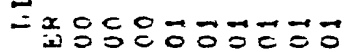

$3++4+7+5$

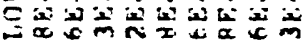

miñ

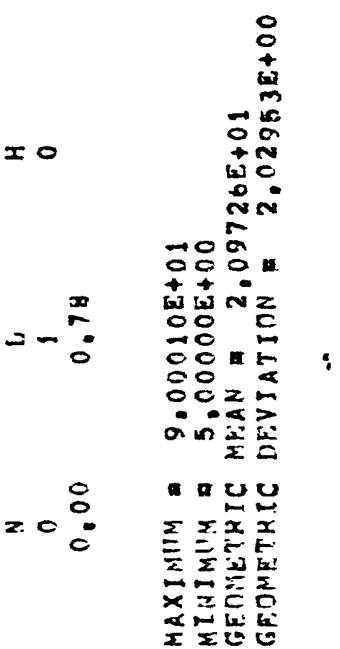


$\stackrel{2}{\frac{1}{2}}$

占
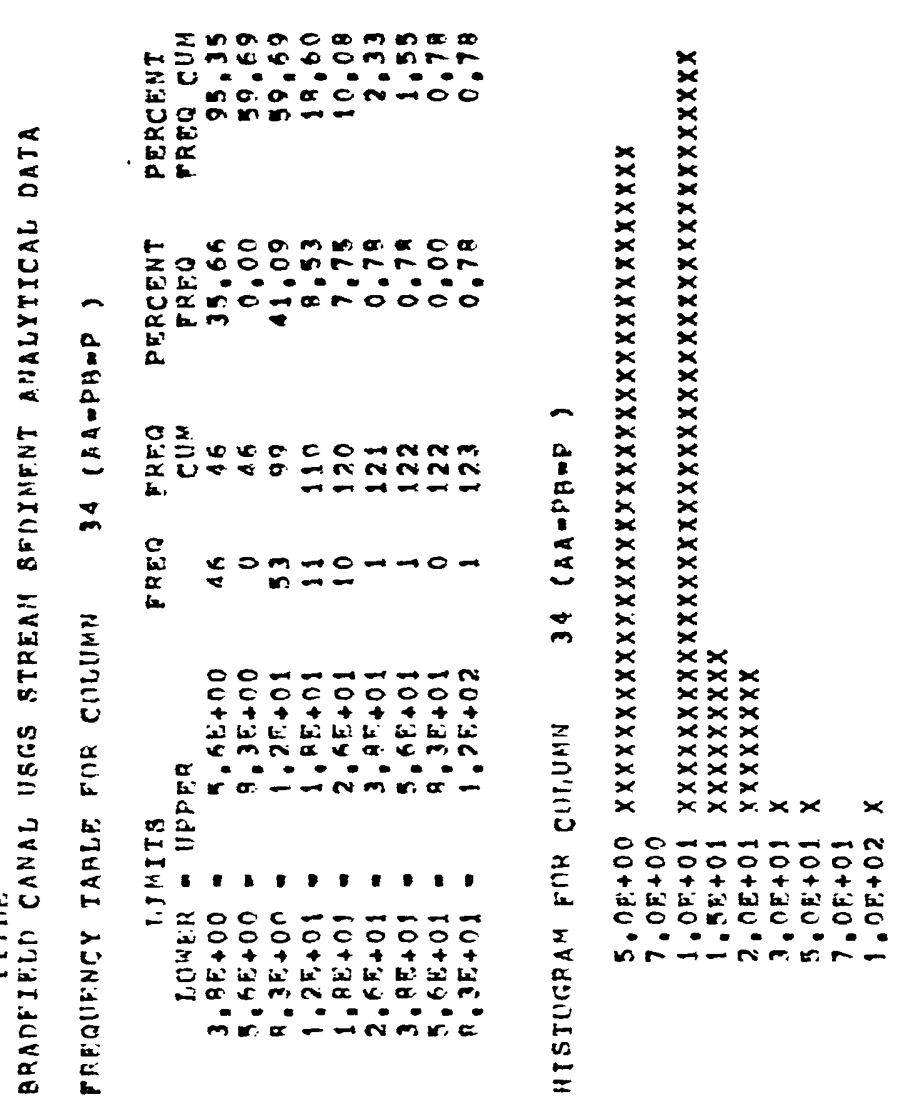

ป

要示

ल)

$\pm=$

这

$00:$

$+0:$

$x \bar{N}$

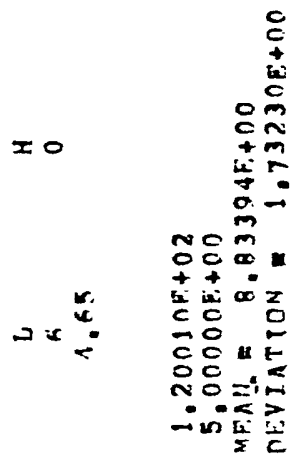

$=0 \stackrel{5}{\circ}$

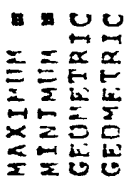




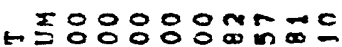
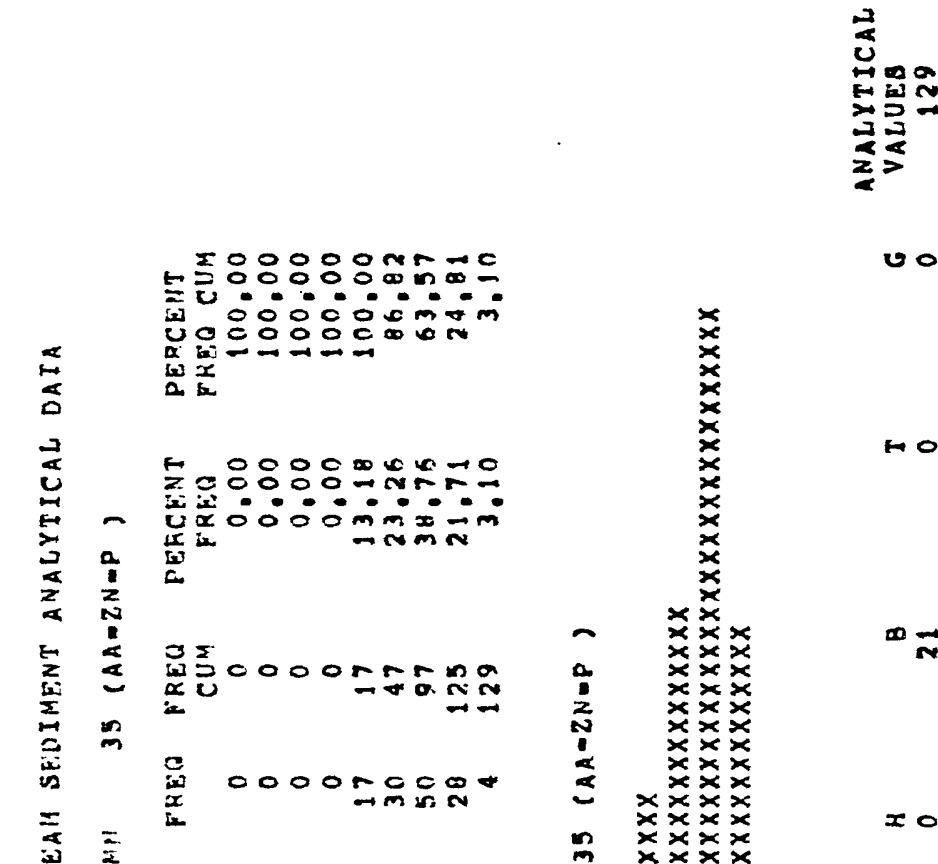

$00:$

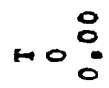

$\infty \vec{n}$

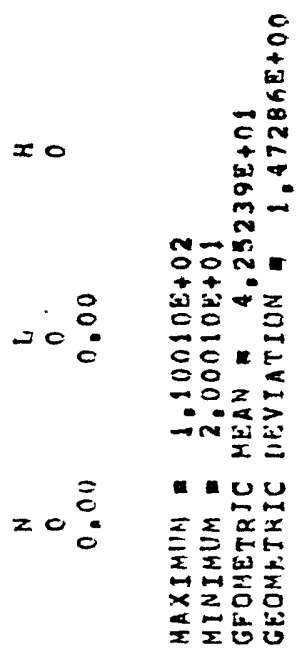




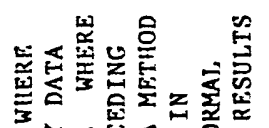

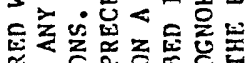

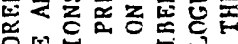

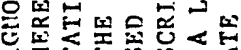

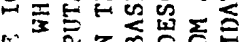

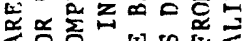

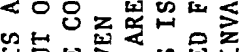

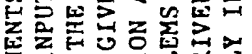

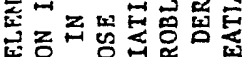

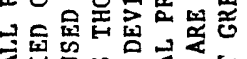

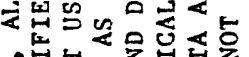

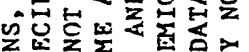

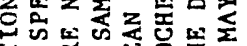

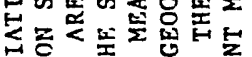

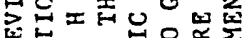

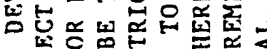

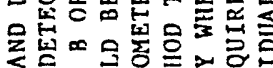

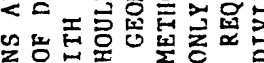

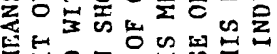

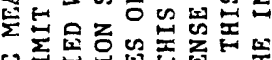

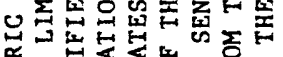

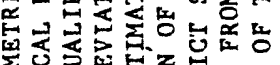

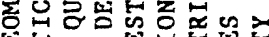

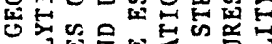

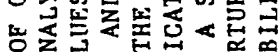

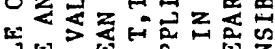

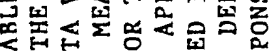

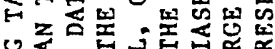

证

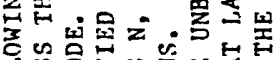

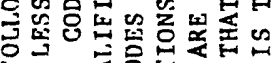

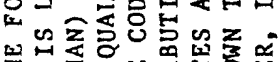

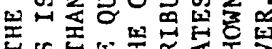

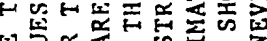

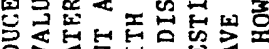

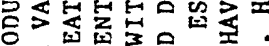

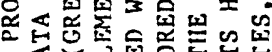

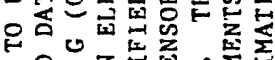

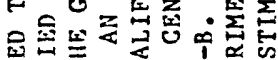

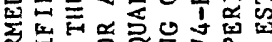

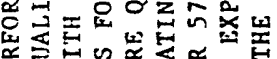

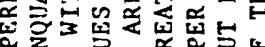

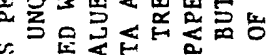

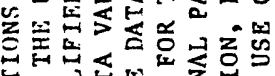

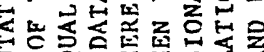

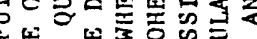

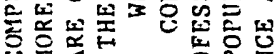

比

至点

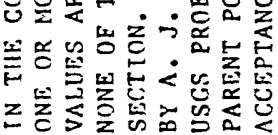

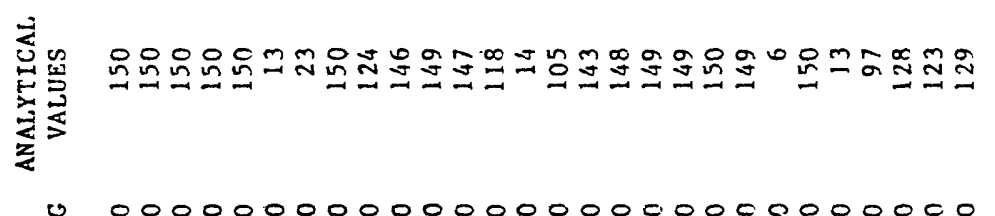

10000000000000000000000000000

W000000000000000000000000000

$\infty 000000000000000000000000 \vec{n} \bar{n} \vec{n}$

$=0000000000000000000000000000$

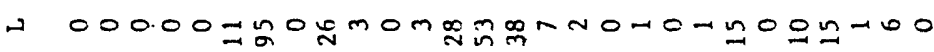

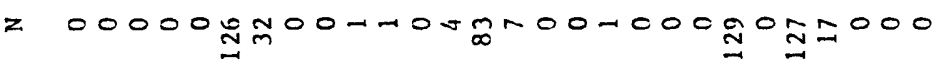

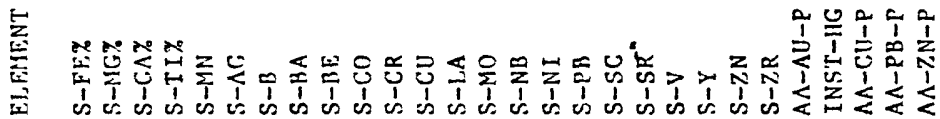




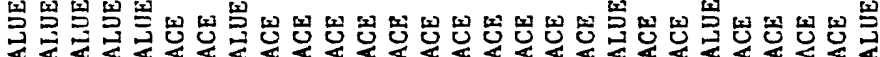

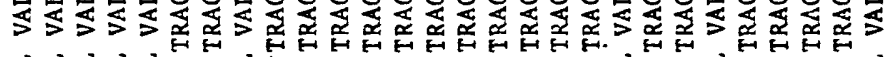

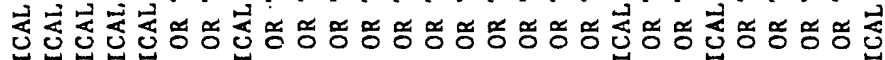

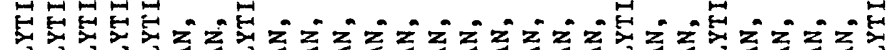

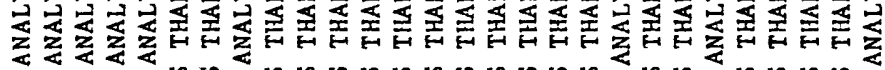

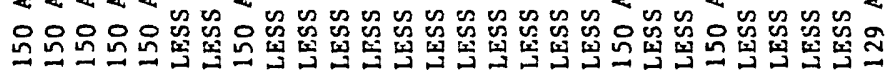

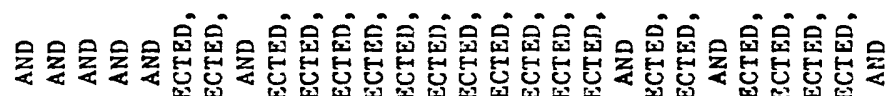

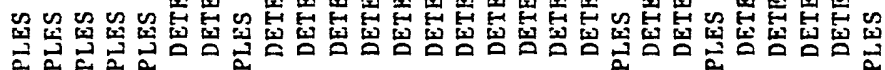

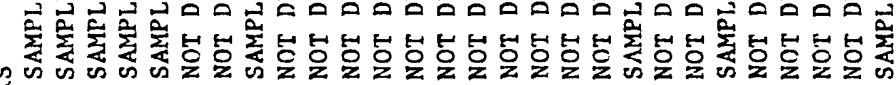

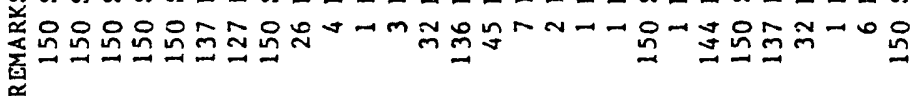

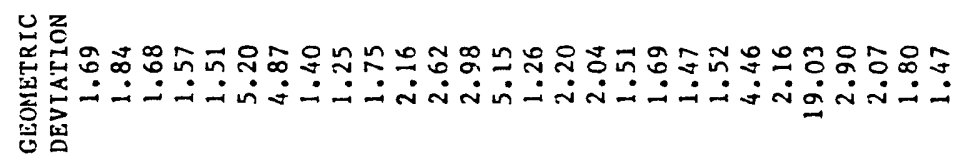

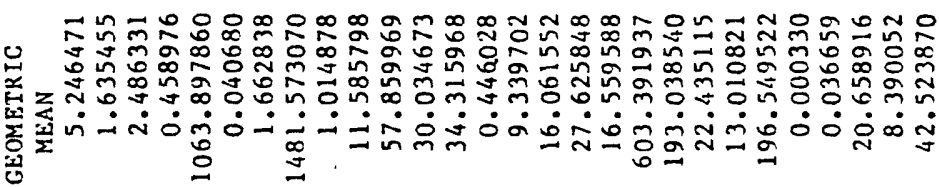

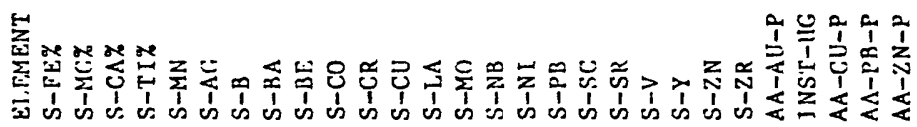




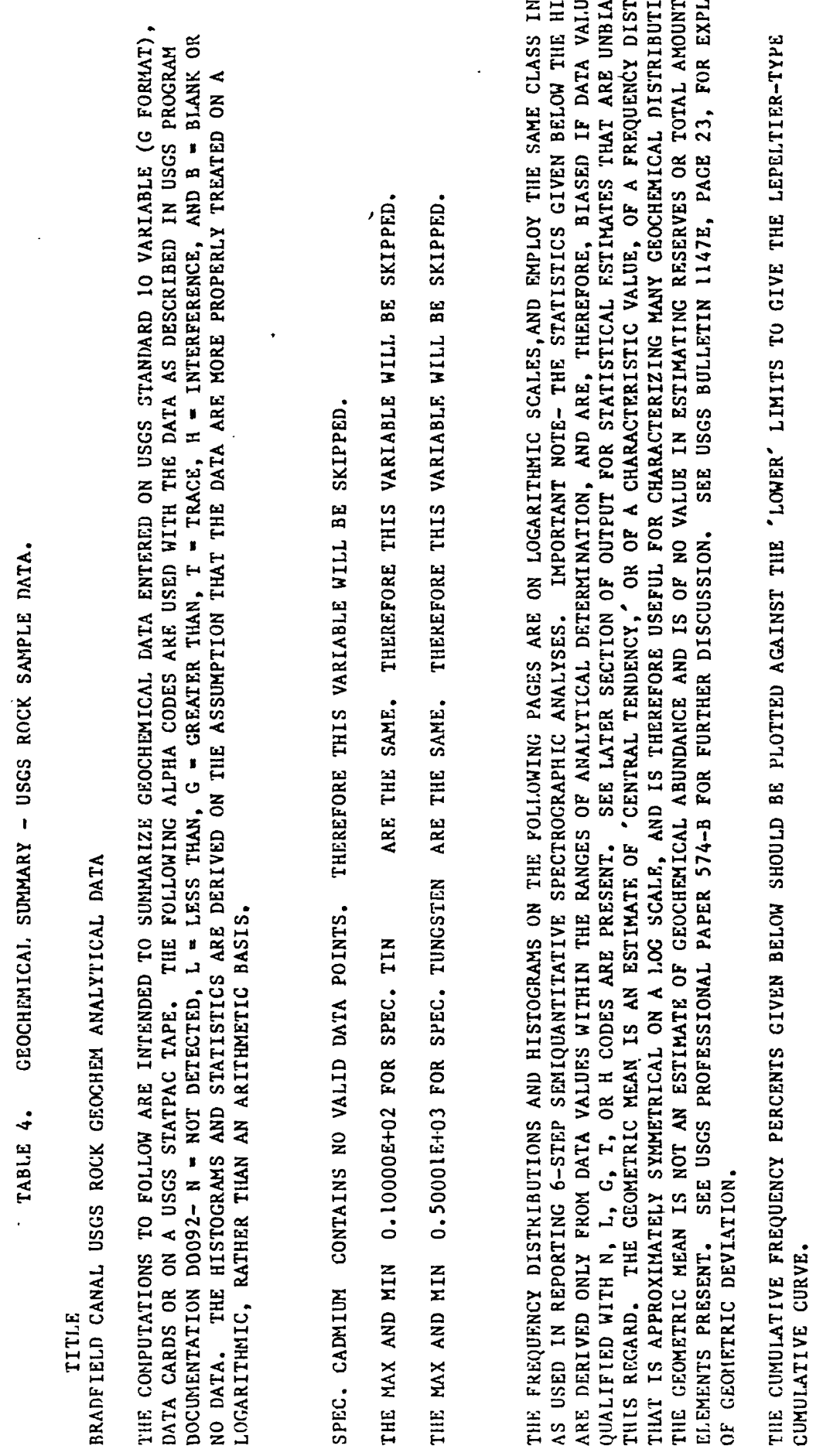

의 


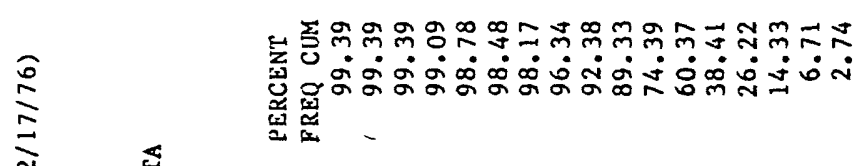

$\operatorname{onn} \stackrel{n}{-1}$

Ho!

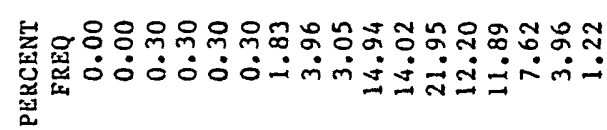

$$
0
$$

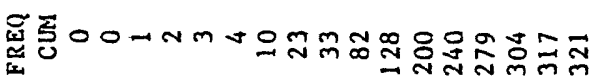
总

$\infty$

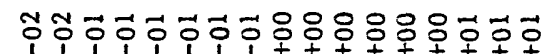

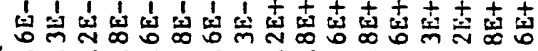

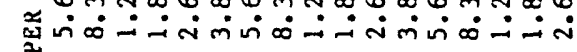
里 辛
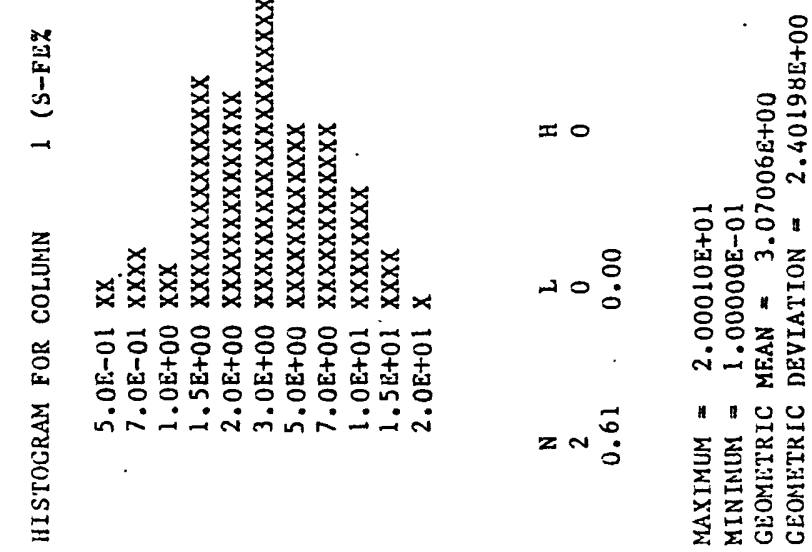


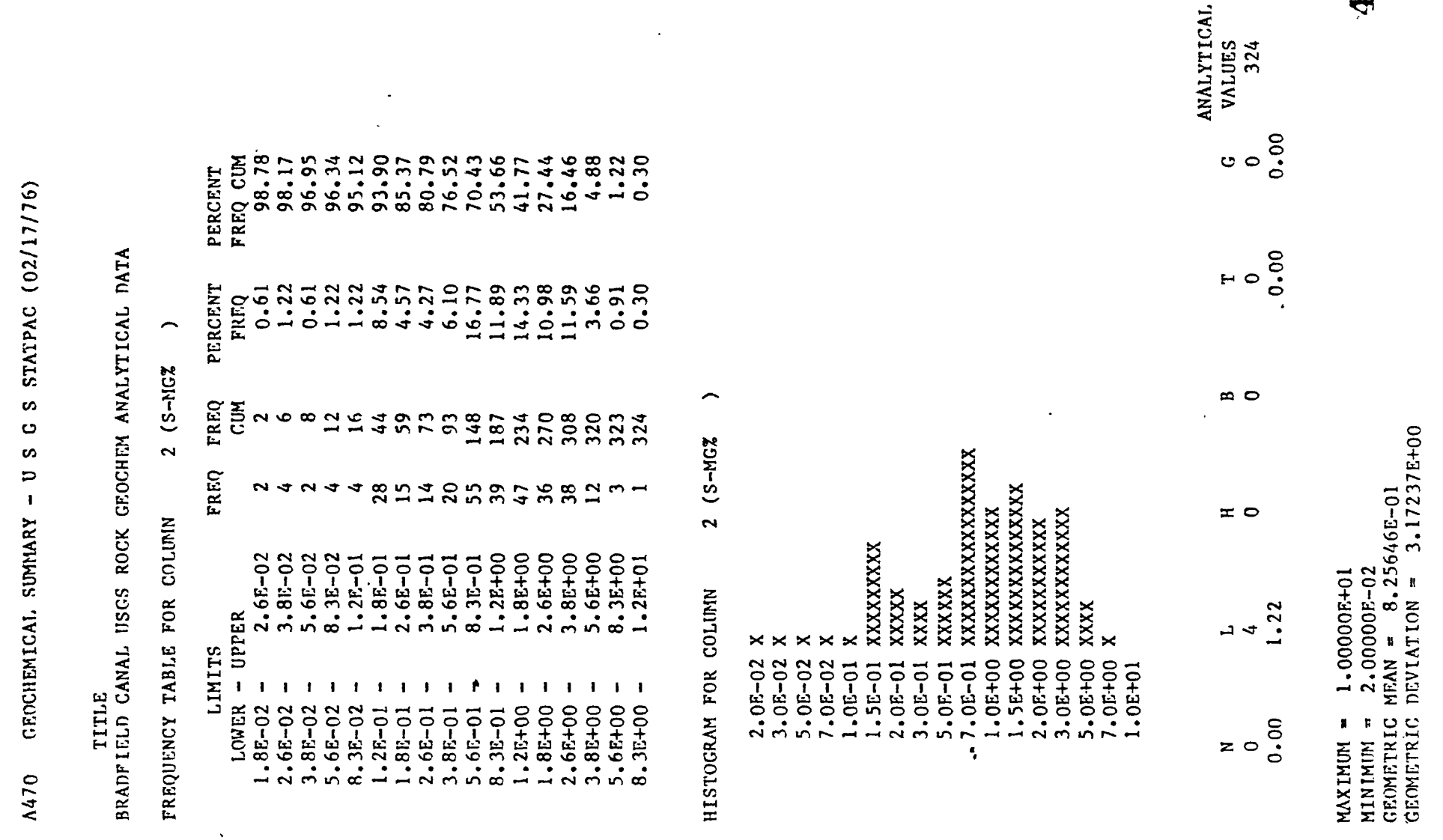


$\frac{0}{\frac{n}{n}}$

感

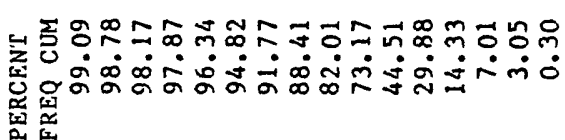

$00 \stackrel{8}{0}$

- $\stackrel{8}{0}$

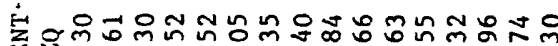

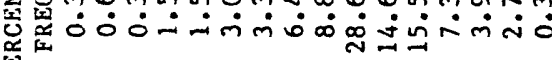

政

这

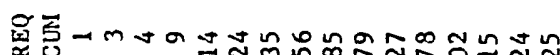

x०

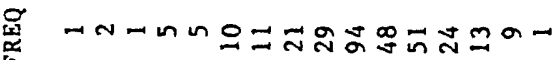

$z$

竞

$m$

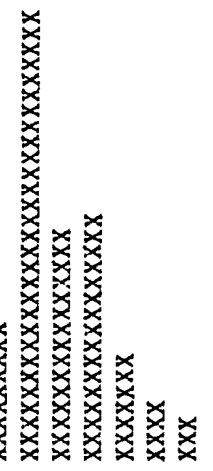

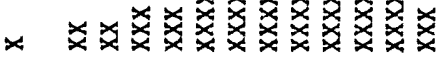

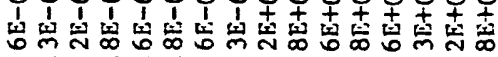

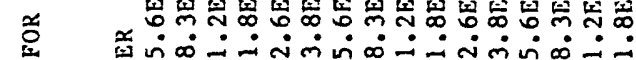

象菅

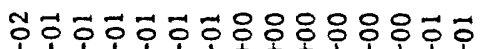

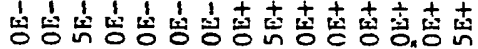

$\infty$

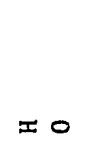

I 0

$=m \ddot{\circ}$

ㅎํำ

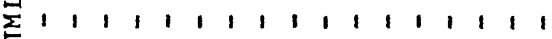

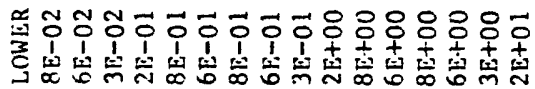

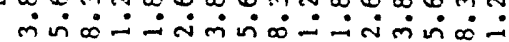

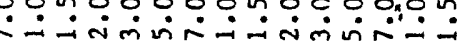

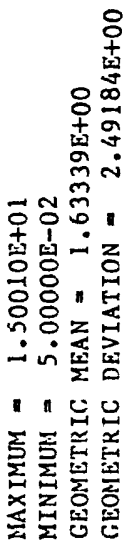


$\frac{2}{n}$

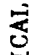

悹蓄

告

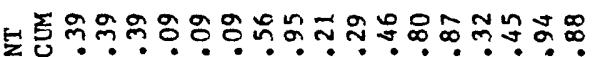

ง $\stackrel{\overline{0}}{0}$

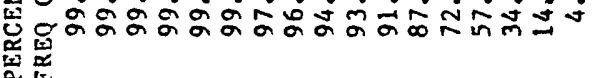

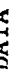

空密

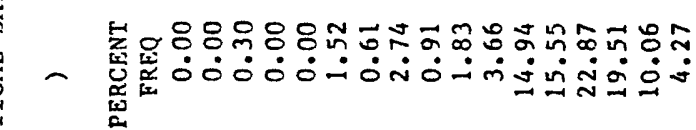

-

Ne

t

$\rightarrow$ N

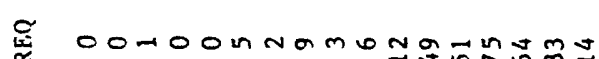

昰

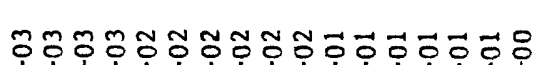

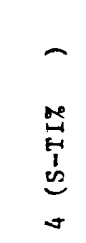

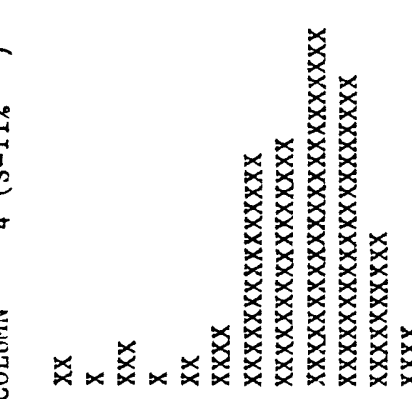

$\infty 0$

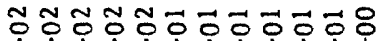

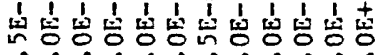

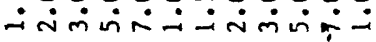
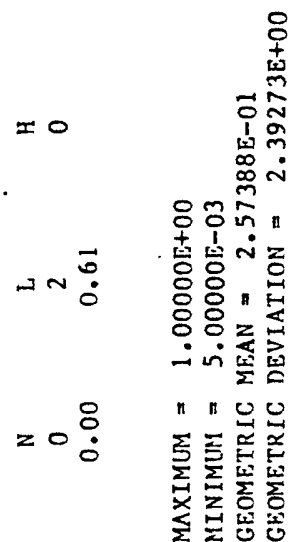

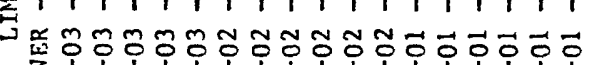

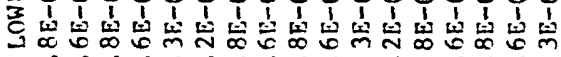

zo: 


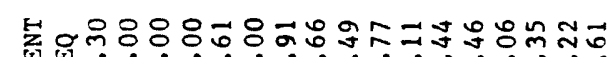

忌

$00 \stackrel{8}{0}$

Ho: 
$\stackrel{\infty}{\frac{\infty}{n}}$

紫

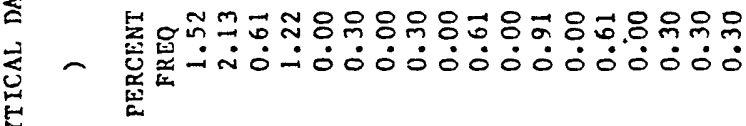

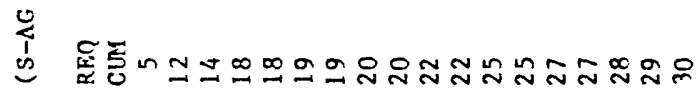

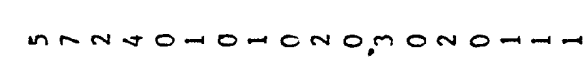
皆絫

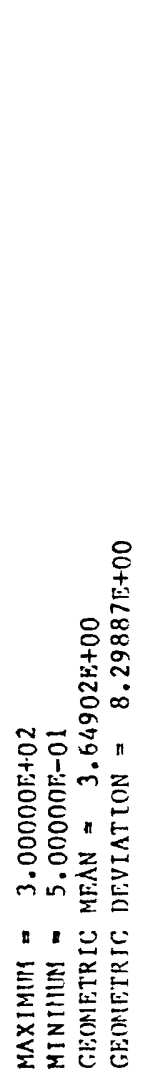



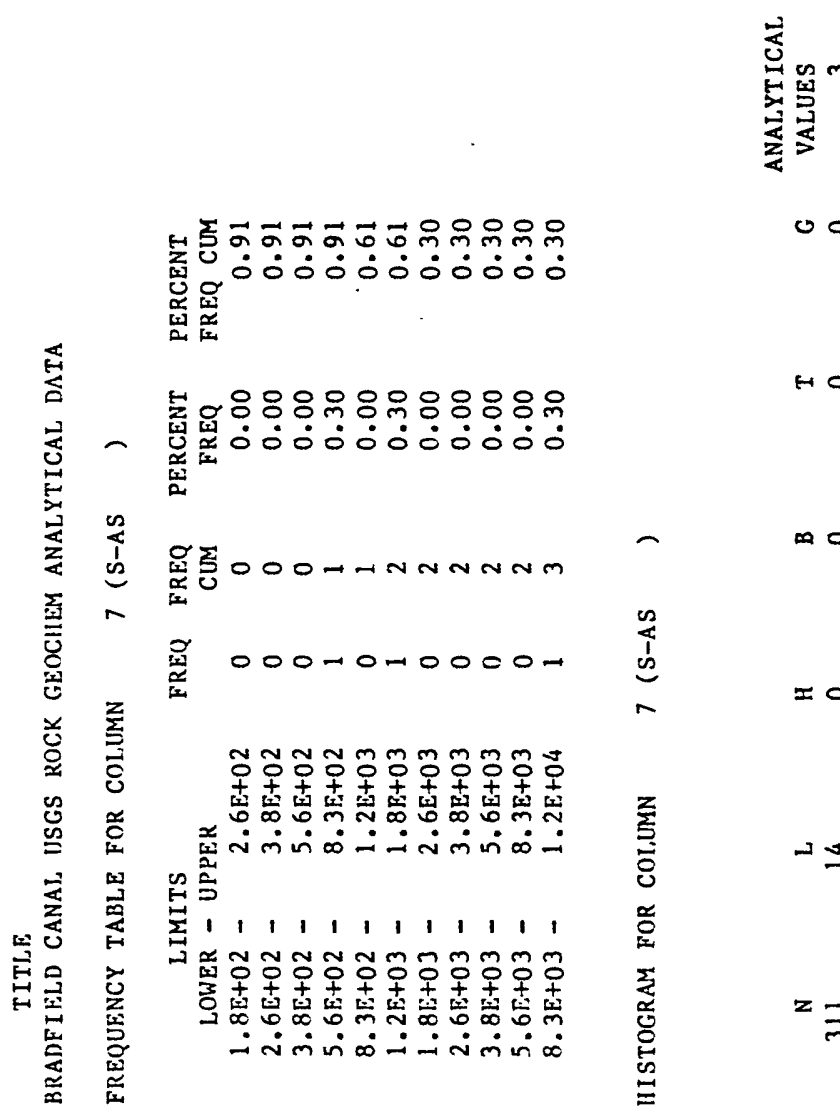

$$
\text { 용 }
$$$$
\text { Ho }
$$

$\infty 0$

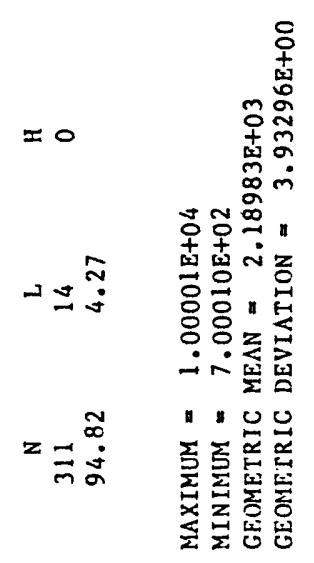


$\stackrel{\frac{N}{2}}{\frac{N}{N}}$

岕

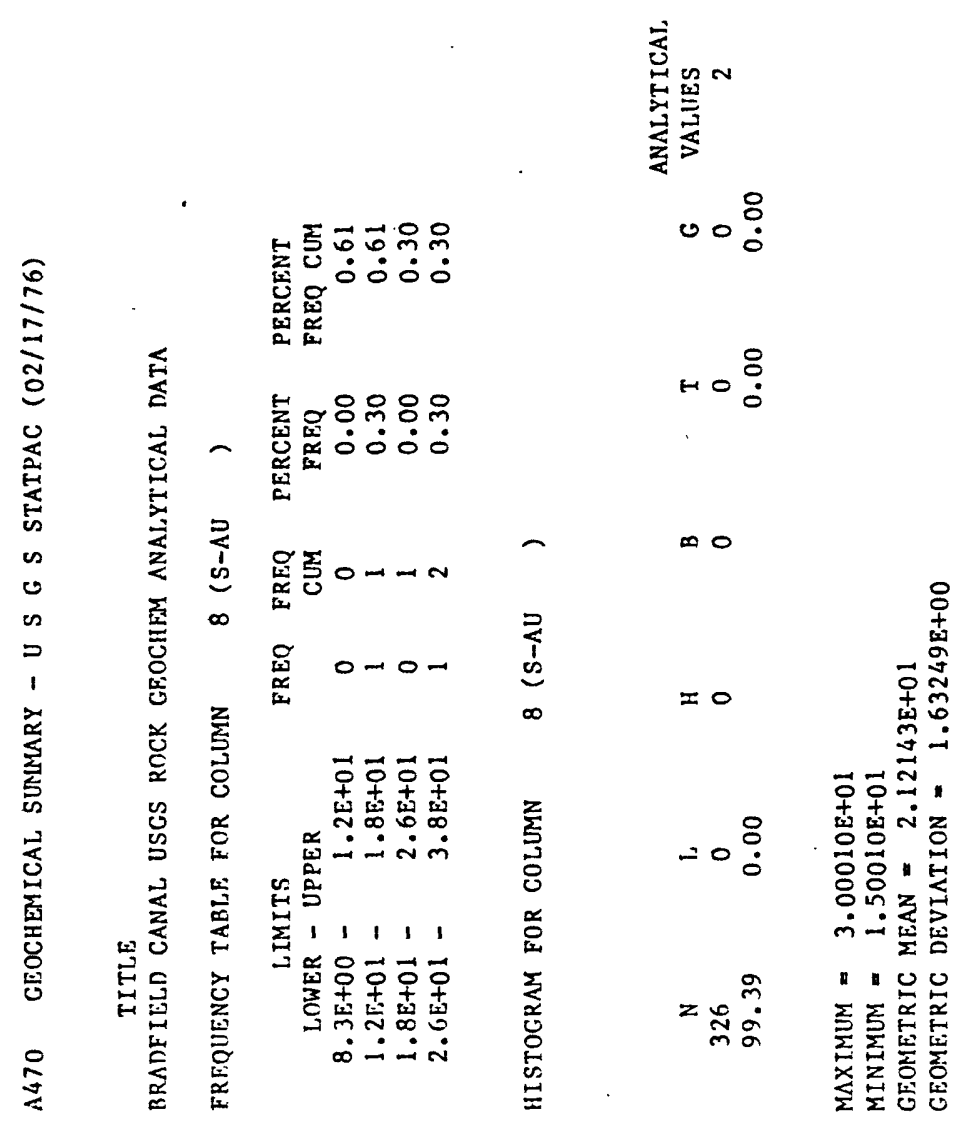




$$
\begin{array}{r}
00 \stackrel{8}{0} \\
\text { H } \\
0: 0
\end{array}
$$

$\infty 0$

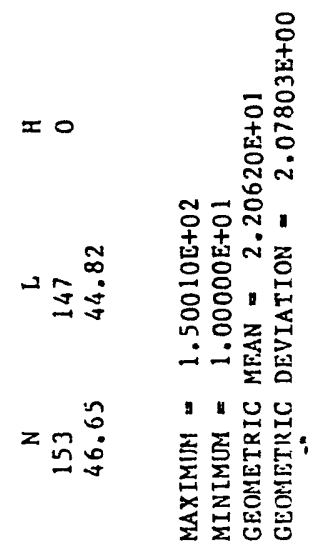




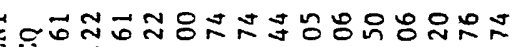

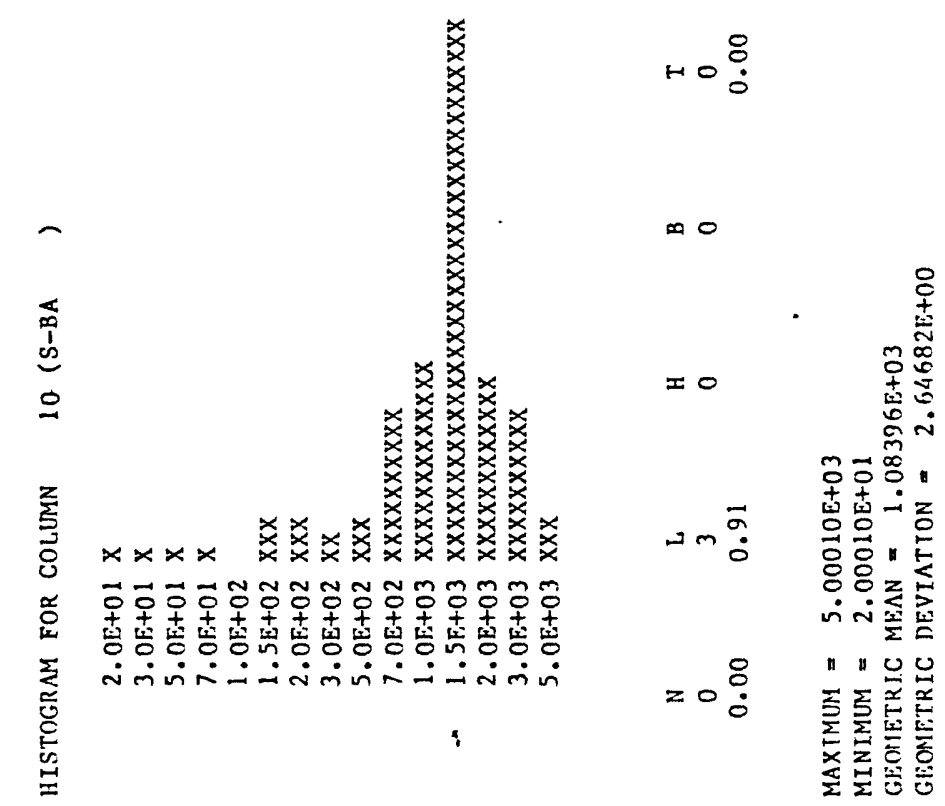




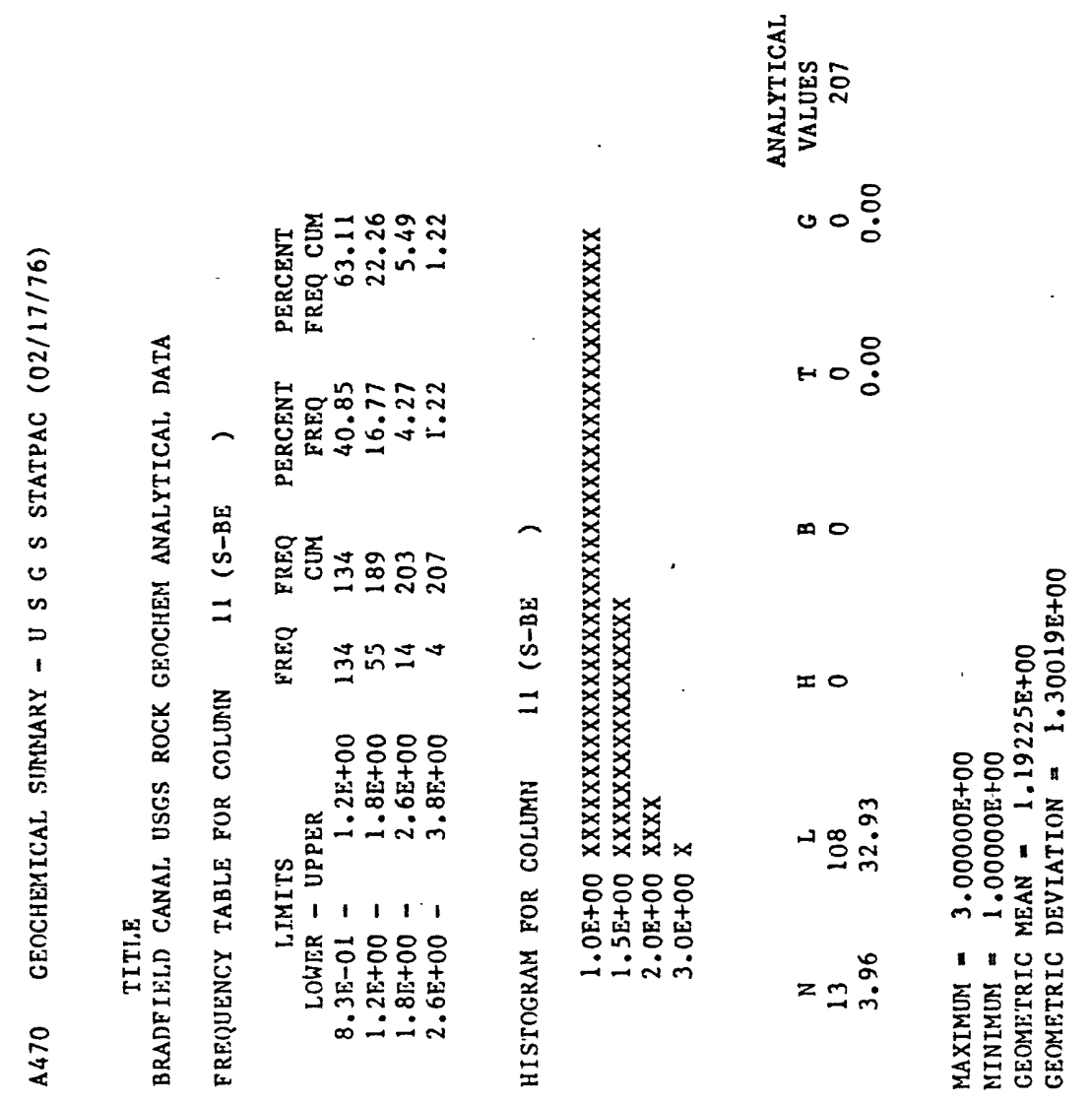


$\stackrel{\frac{2}{2}}{\frac{2}{n}}$
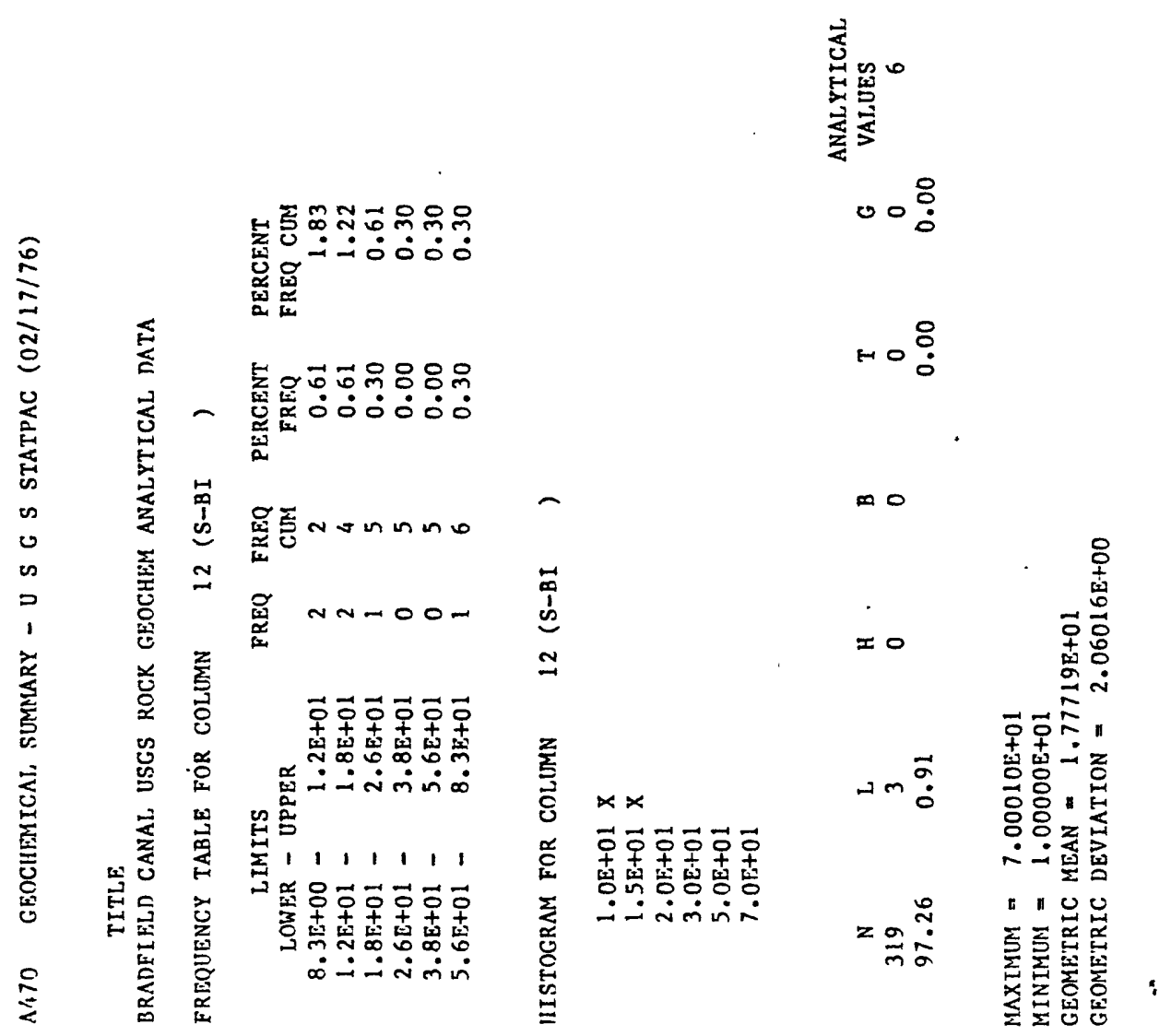

ถึ 


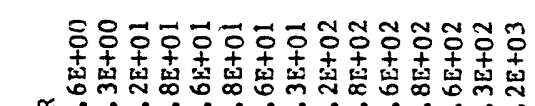

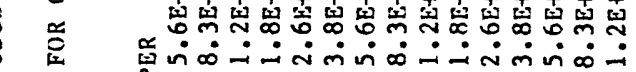
就

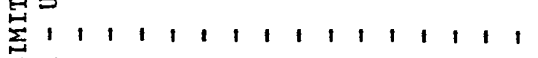

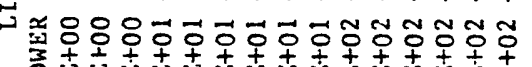

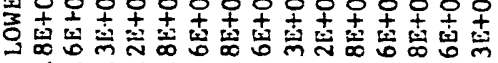

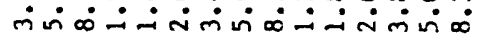

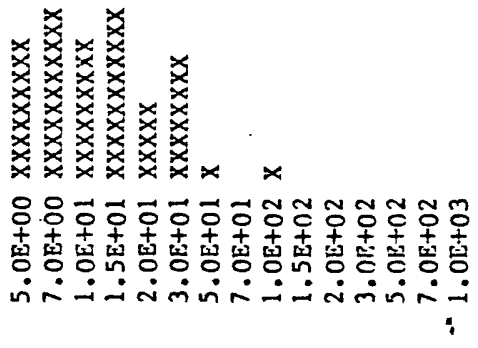

$\infty$<smiles>O=O</smiles>

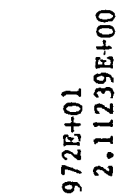
mㅇํㅇ त्ञ

$\pi \cong$ 
$\infty$

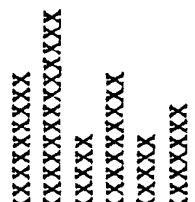

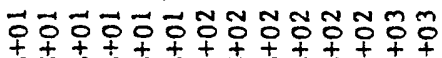

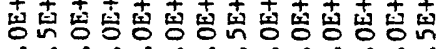

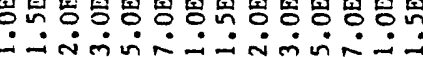




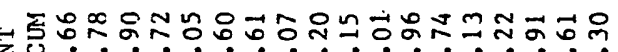

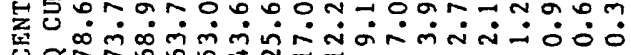
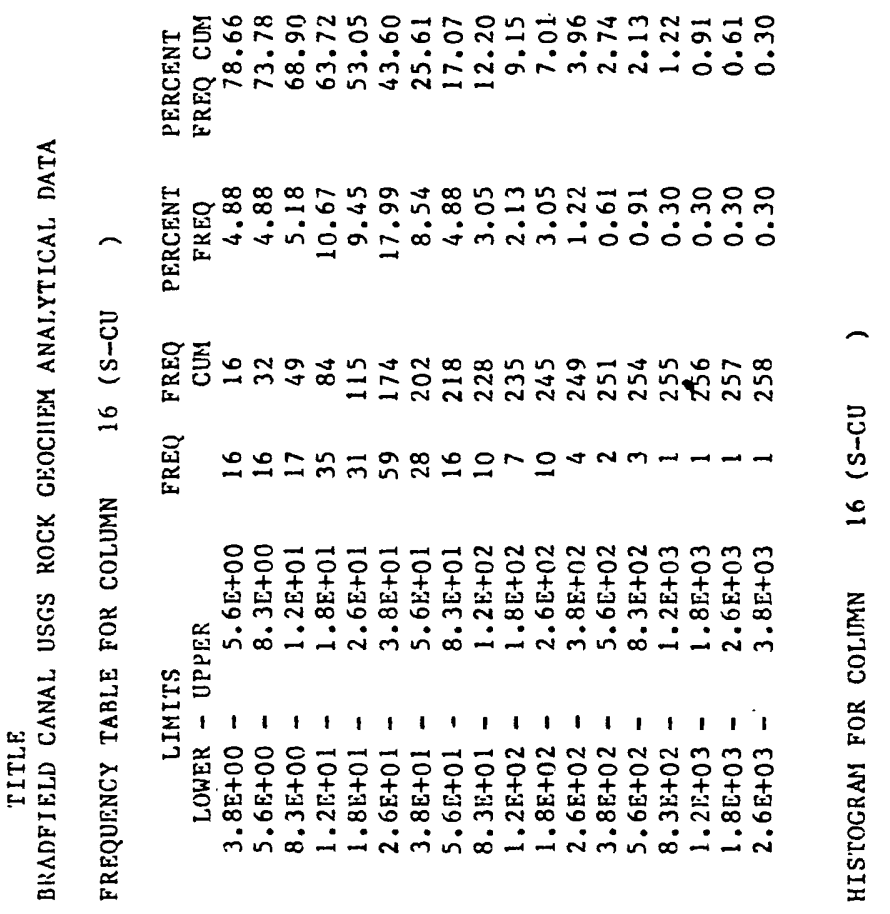

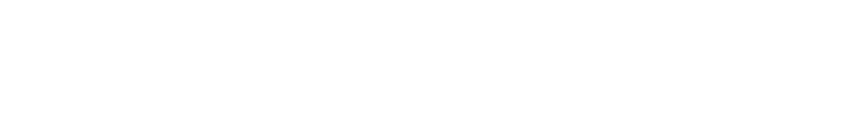

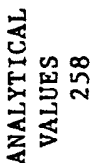

$\operatorname{Non}_{1}$ $00 \stackrel{8}{\circ}$ Ho:

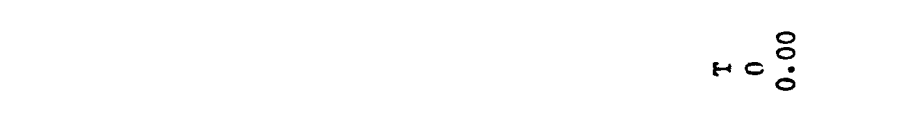

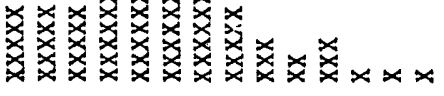

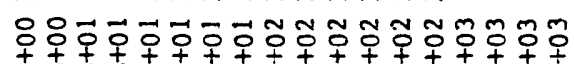

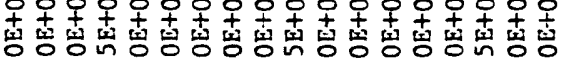

$\infty 0$

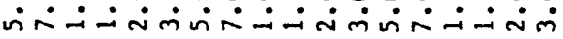

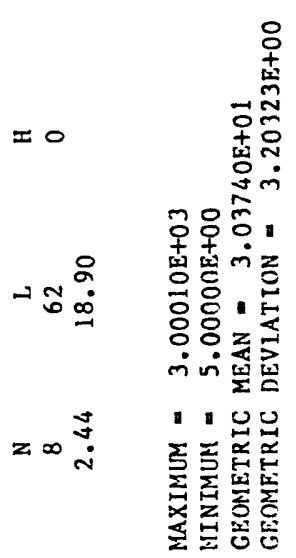




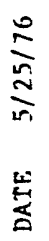

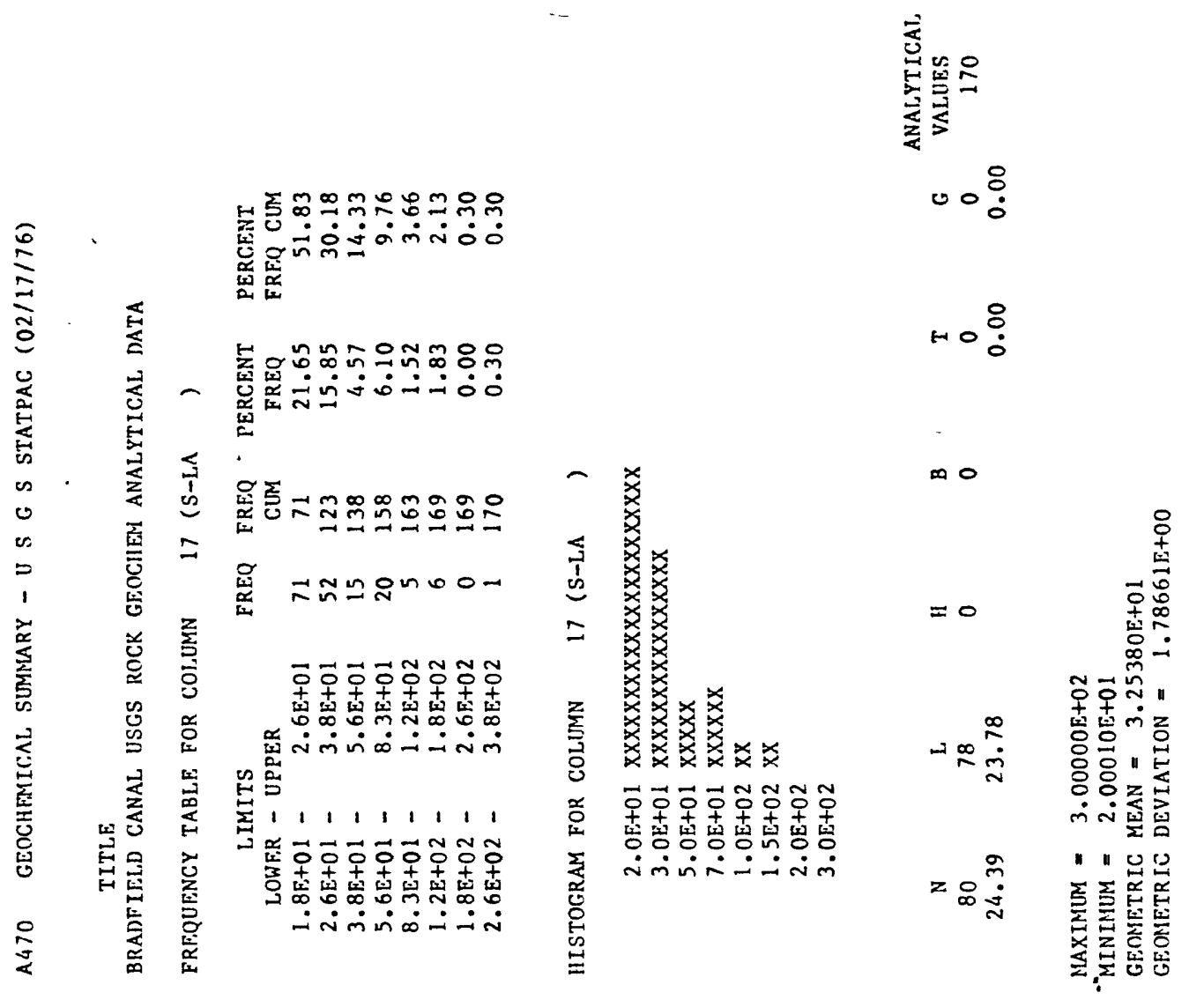




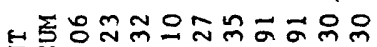

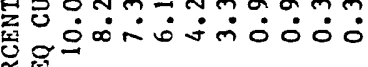

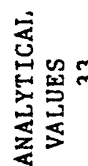

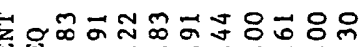

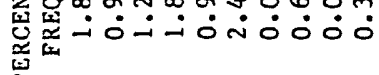

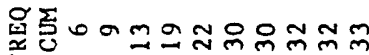
I D OMUnono$-\quad \sum_{j}^{\infty}$ $\infty$

๓ 0

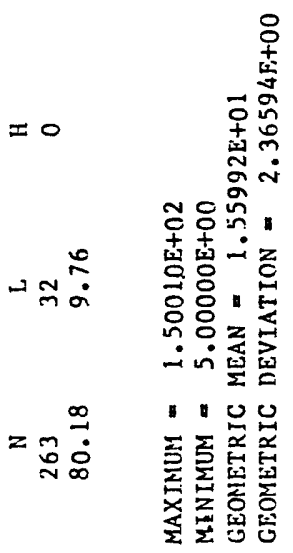



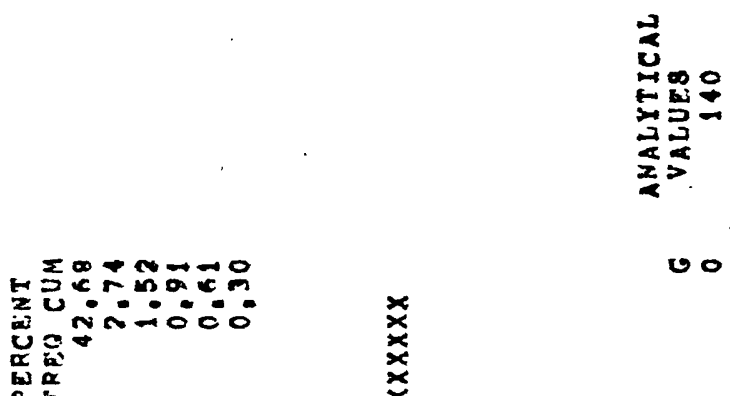

$$
00 \stackrel{\circ}{\circ}
$$

$$
+0 \stackrel{8}{0}
$$

a 0

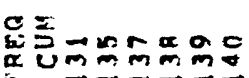

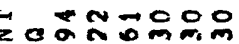

卧: - . - .

然 (i)
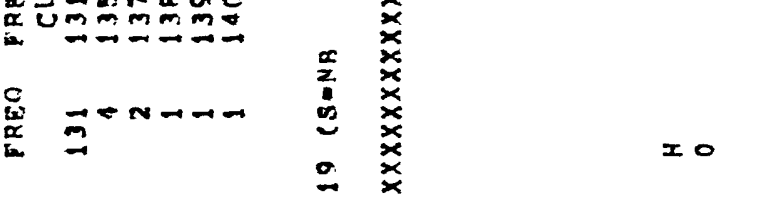

Io
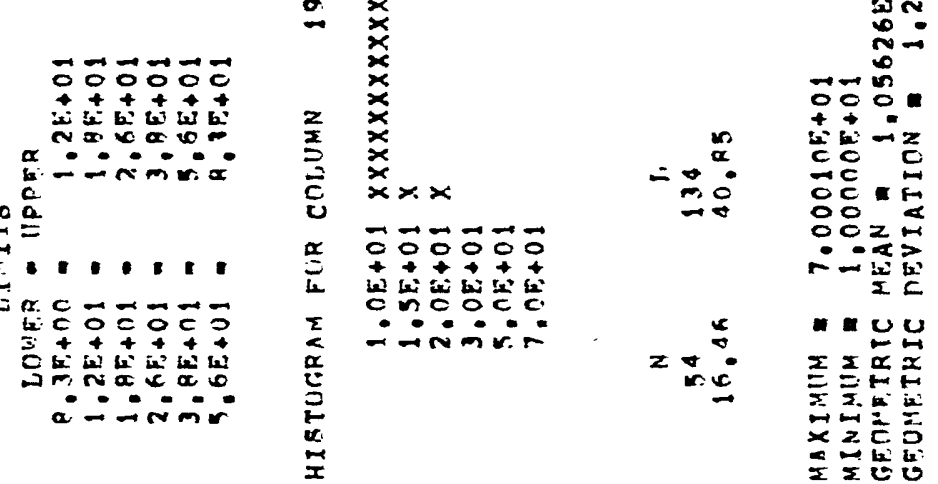

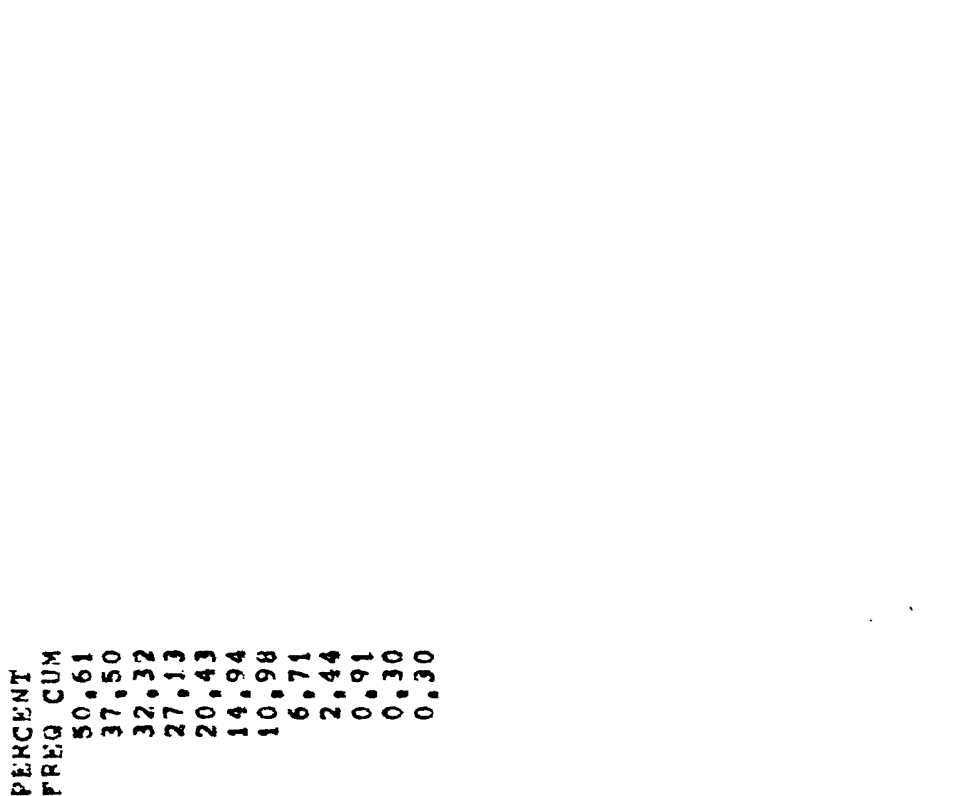

$00:$

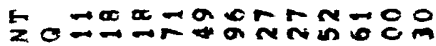

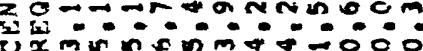

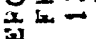
a.

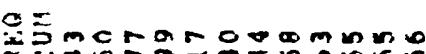

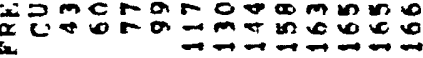
ก

离

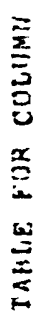

ㄴ.

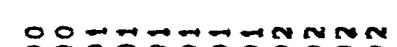

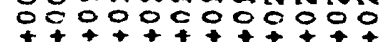

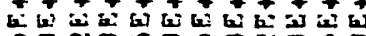

$x=n \infty c \infty c m n \infty \infty$

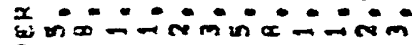
$\infty \stackrel{2}{=}$

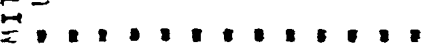

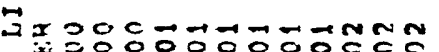

si

$5++4+t+t+t+t$

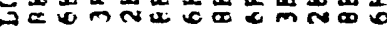

mina

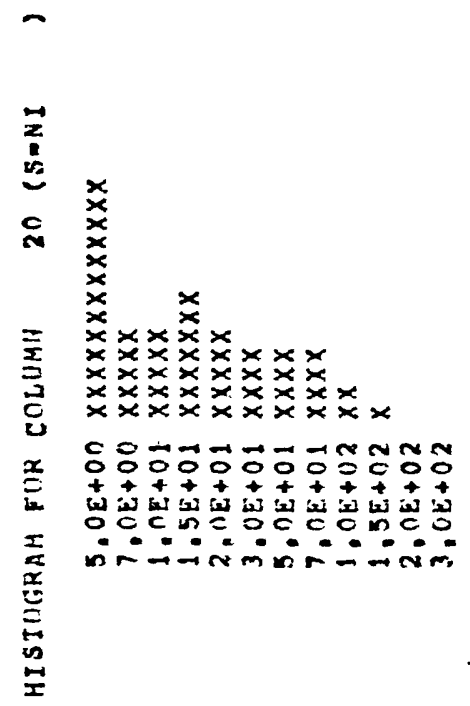

$\infty 0$

$+\infty \stackrel{0}{0}$

c

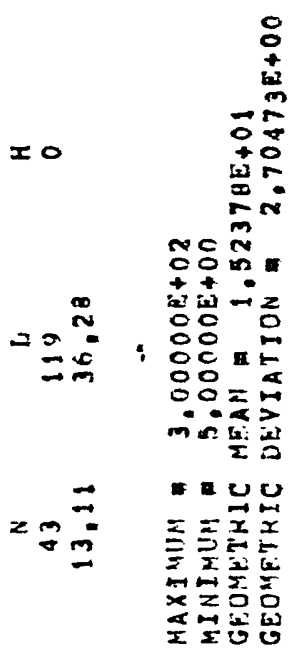




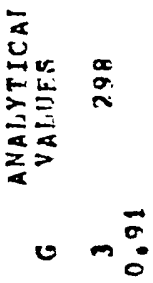

$+\quad \stackrel{0}{0}$

$\stackrel{5}{a}$

$\infty 0$

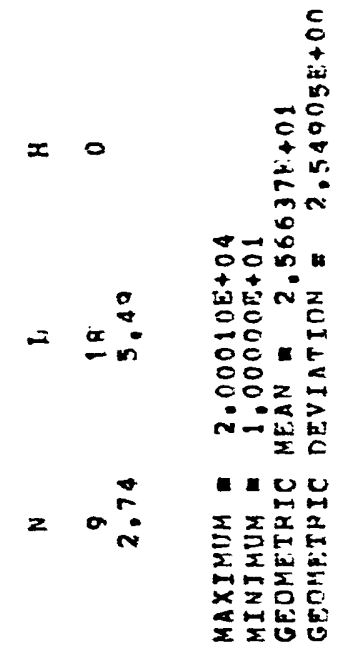

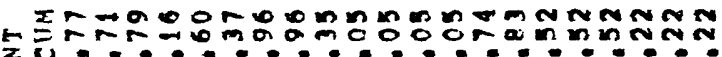

U w in

a.

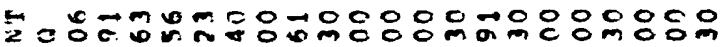

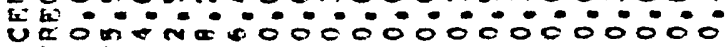
$\sum_{i=0}=0$

a.

a.

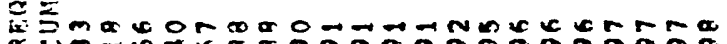

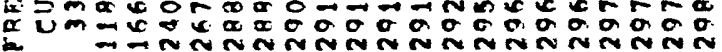
N

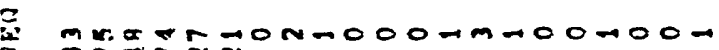
b. $\max \pi \pi$

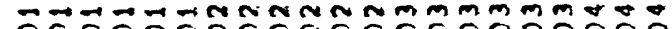

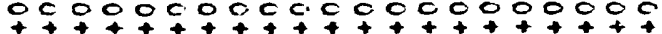
死

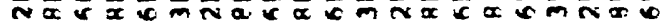
绐竞

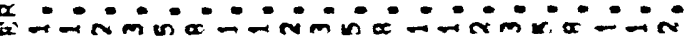

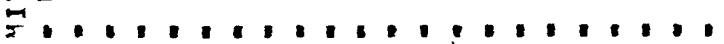

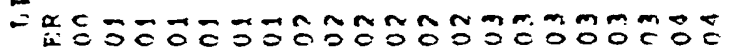
然,

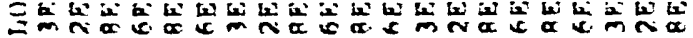
a- inma-

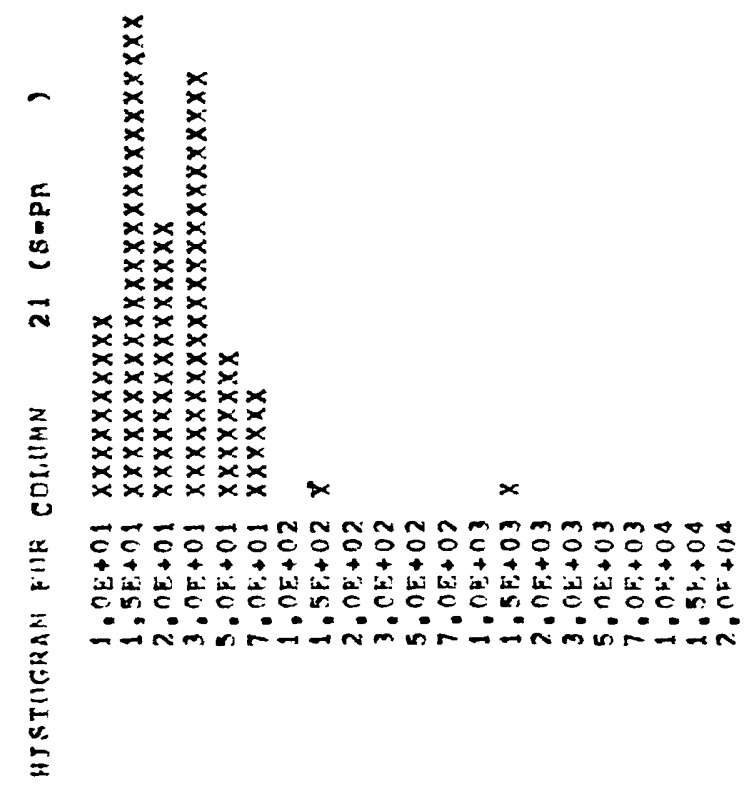



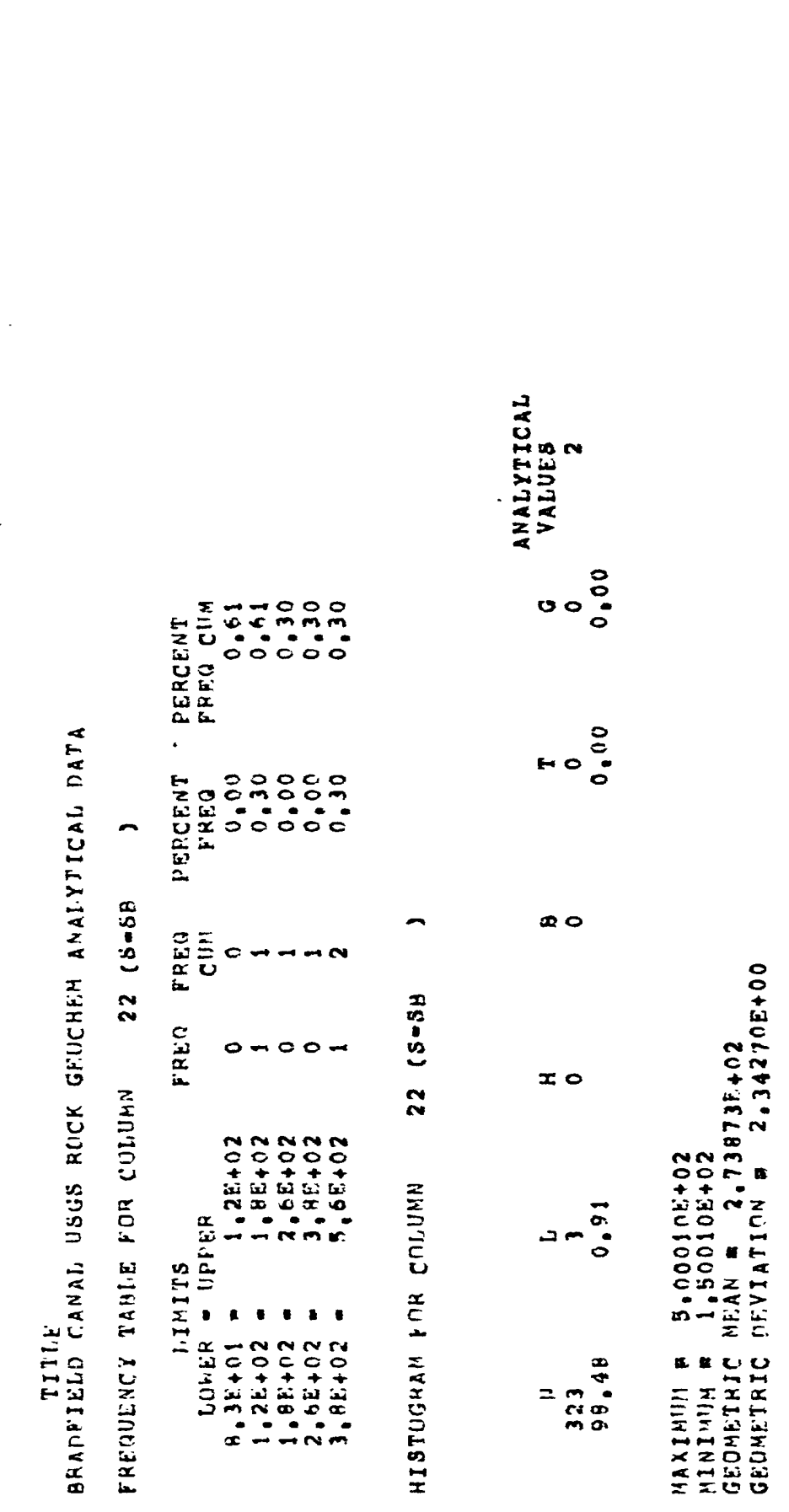


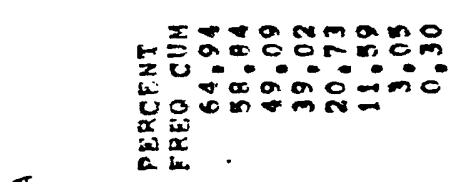
a
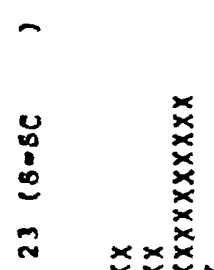

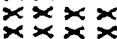

$x$
$x \times x$
$x$

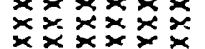

xxxxx

$x \times x \times x \times x$

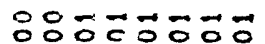

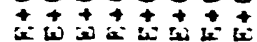

cocacos

$\infty 0$

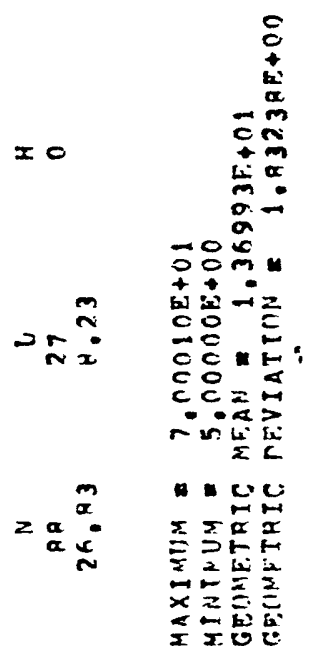




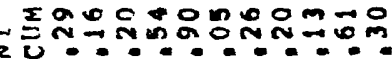

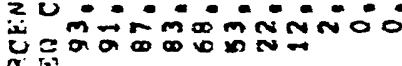
is

는 은

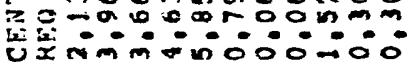

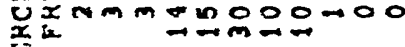
as

$+0 \stackrel{\circ}{0}$

$\infty 0$

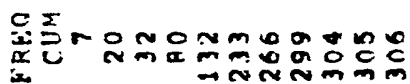
is

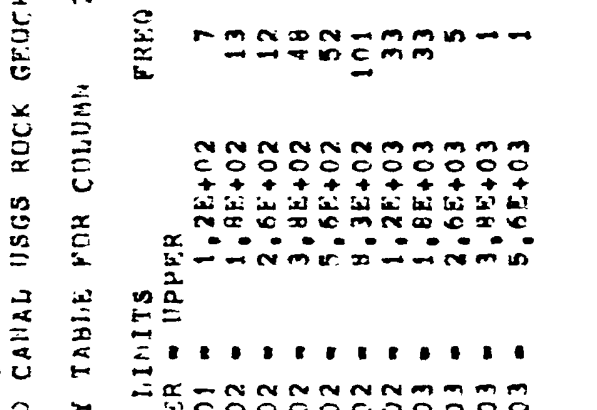

눈요

空可

고

$x>$

$00 \stackrel{\circ}{\circ}$ 


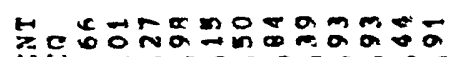

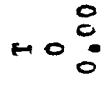

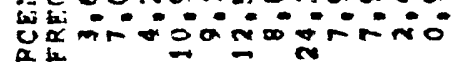

$00 \stackrel{0}{0}$

a

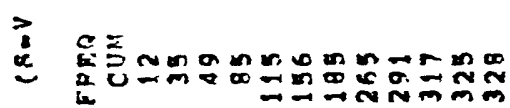

-
$\vdots$
0

$\overrightarrow{0} \overrightarrow{0} \overrightarrow{0} \bar{O} \overrightarrow{0} \approx N N \approx$ $+t+t+t+t+\frac{1}{t+1}$

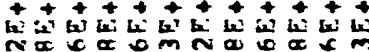

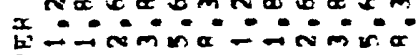
$\infty$

究 $E=$

$=1.1 .1 .1 .1 .1$.

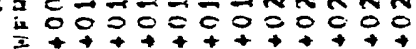

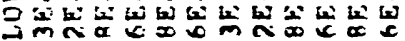
- - inimánimin

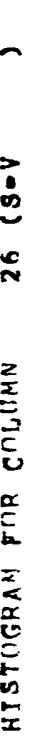

$\infty 0$

xo

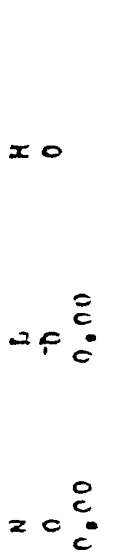



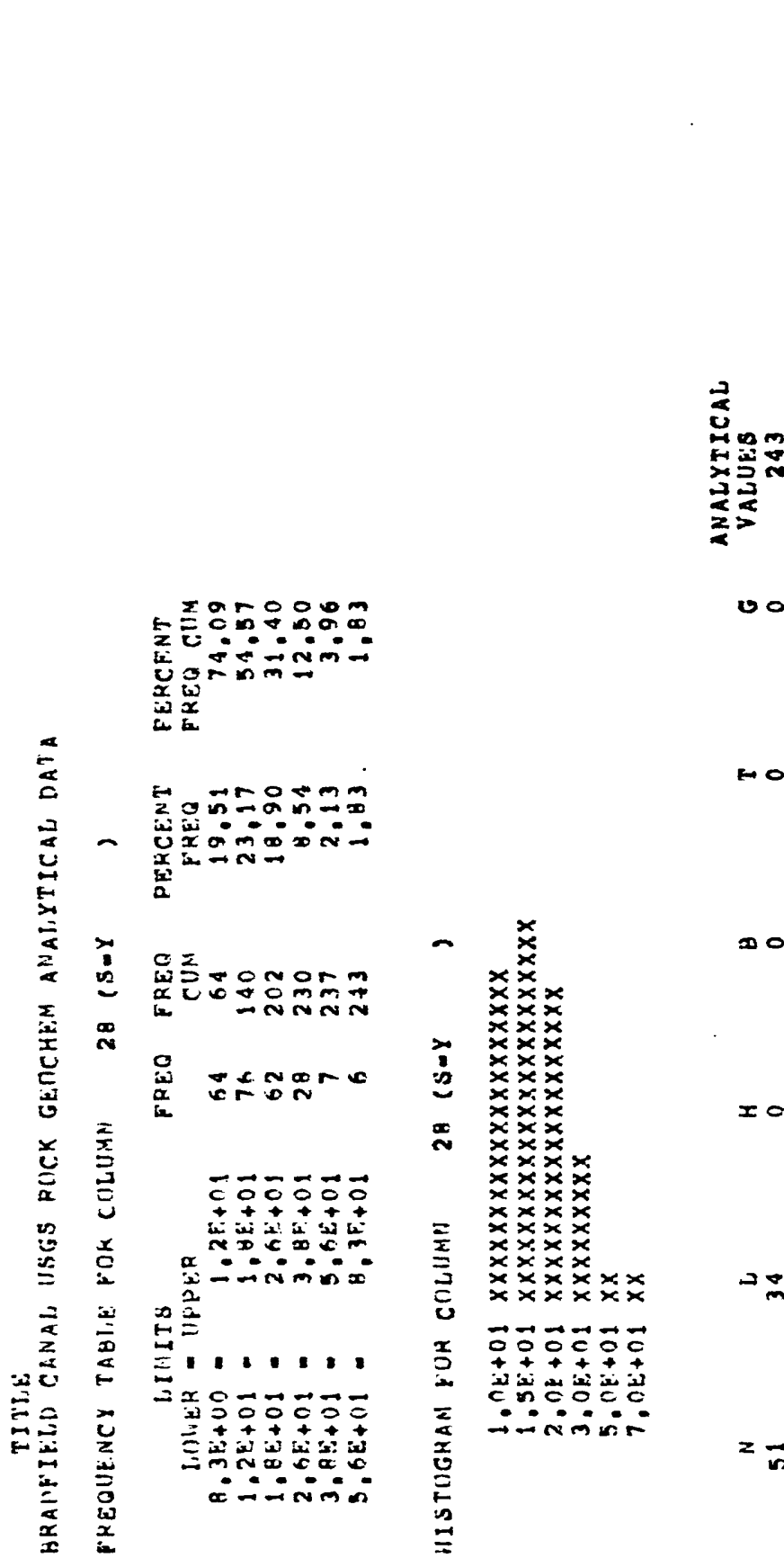

0

$00:$

$10:$

$\infty 0$

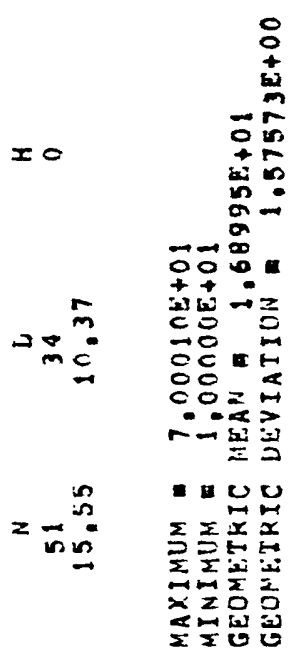


I

$\underset{c}{\stackrel{a}{c}}$
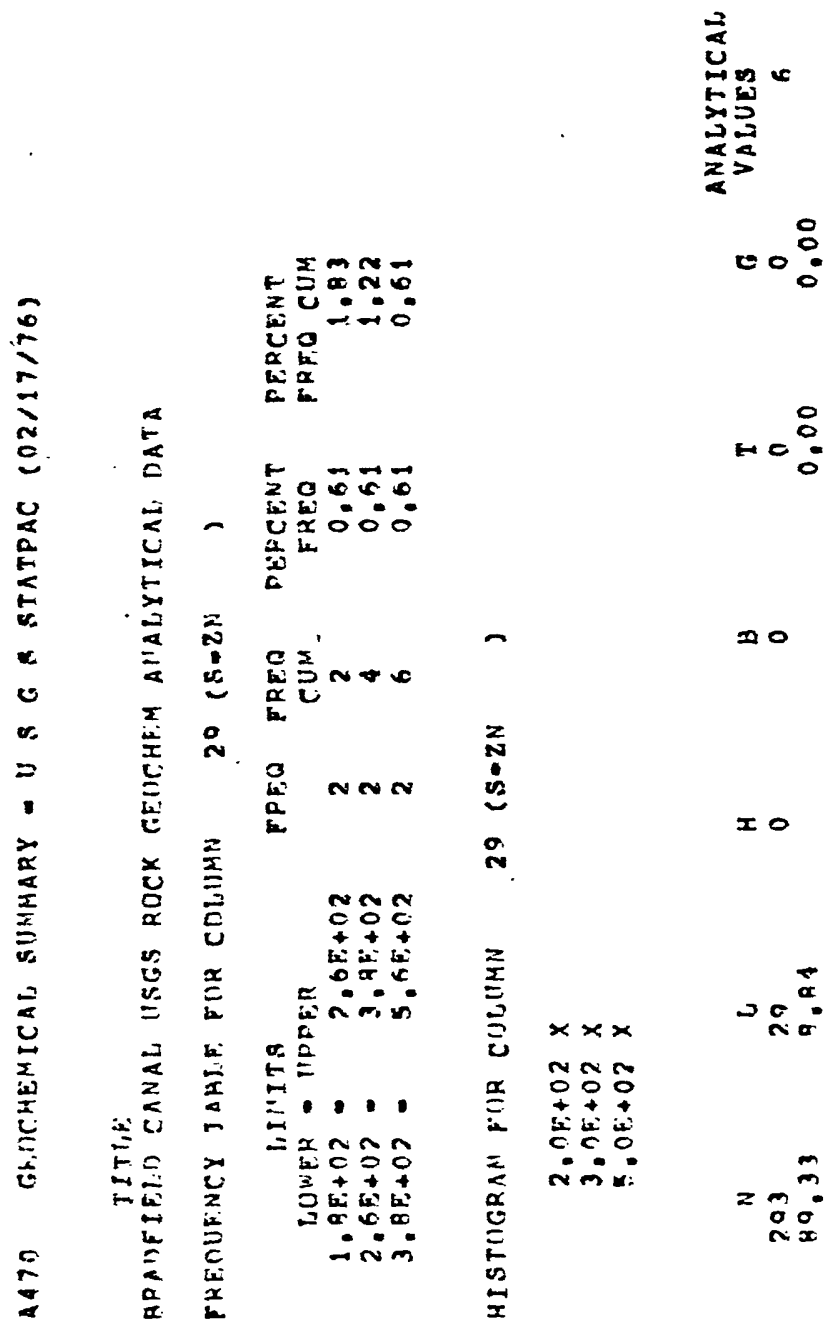

no

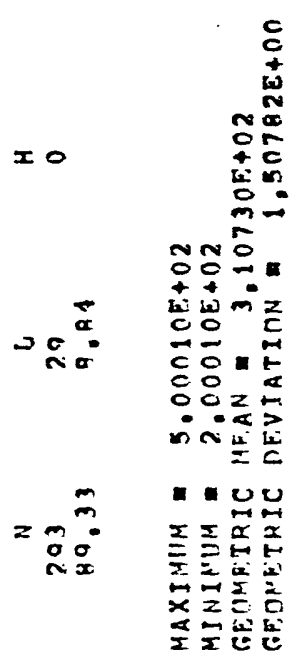




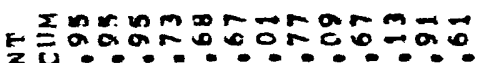

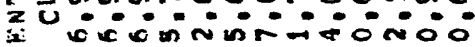

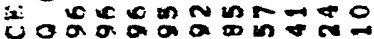

$\frac{x}{x}$

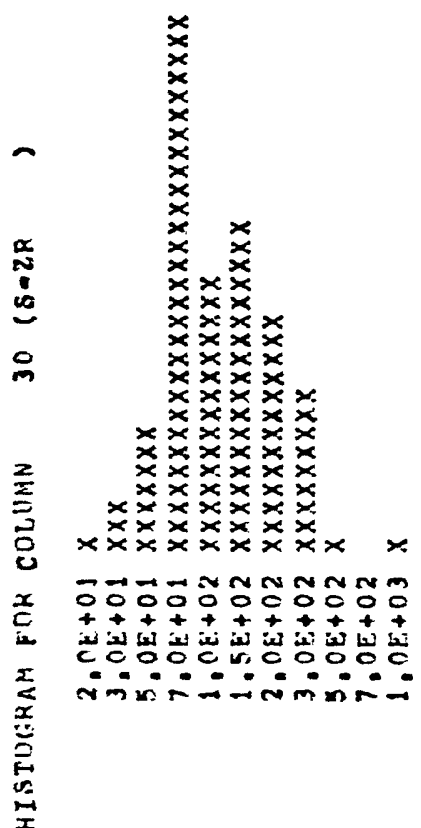

$\infty 0$

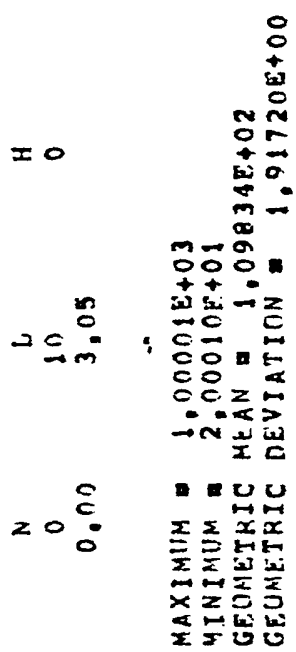




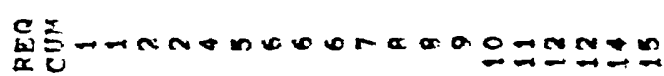

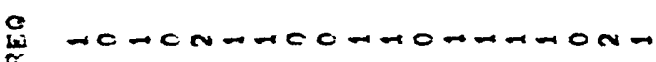

$=0$

$$
=0
$$




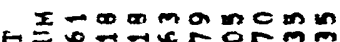

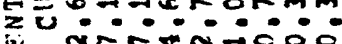

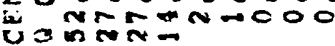

is $x$

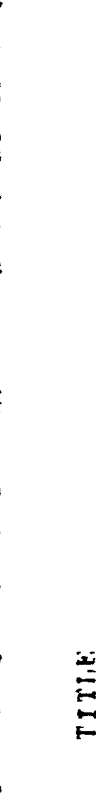

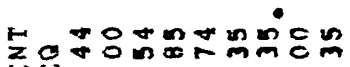

3

$\rightarrow$

넉은

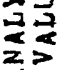

$00:$

I

¿ $\quad$ is

$\rightarrow \quad 0 \quad 3$

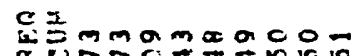

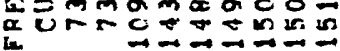

momminm-

so

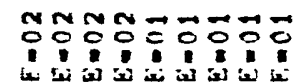

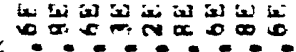

ninisand

$\sum$

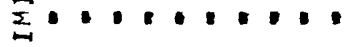

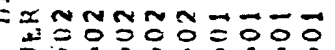

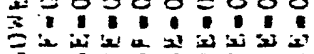

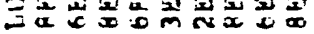
- imix

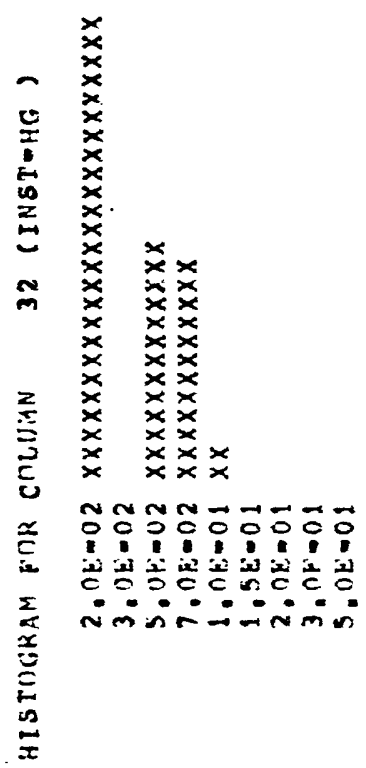

$\infty$

$\rightarrow 0 \div$

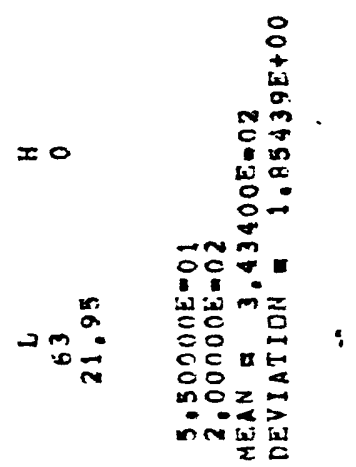

$2 \underset{m}{\infty}$ 
$\sum_{n}^{\infty}$

桌

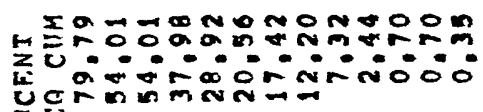

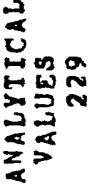

娄究

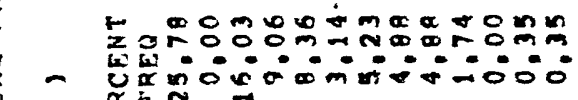

$\frac{\pi^{2}}{2}$

$\vdots$

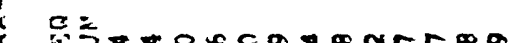

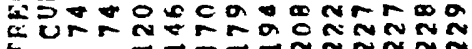

$3+000+0 n+\forall$ nom

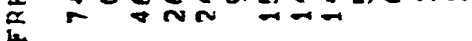

约

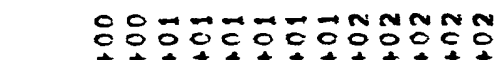

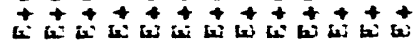
$0 m \pi x x x m x x x$

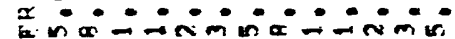

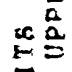

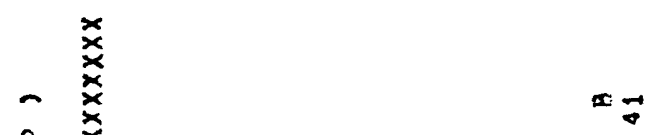

$H=\stackrel{\circ}{:}$

$00 \stackrel{\circ}{\circ}$

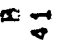

I 0

$m$

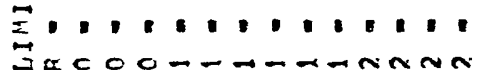

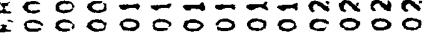

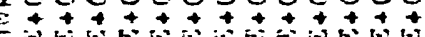

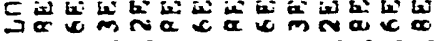

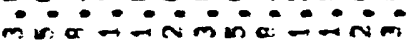

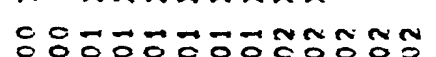

+t+to웅ㅇㅇ

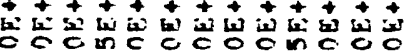

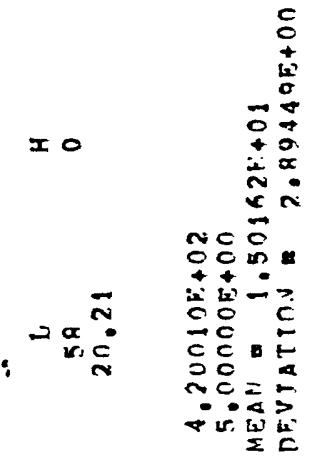

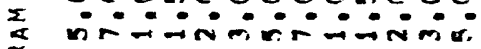

$=0 \stackrel{\circ}{0}$

avu

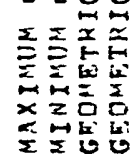




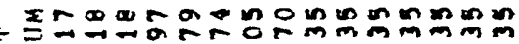

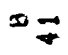




$$
00:
$$$$
\text { wo }
$$

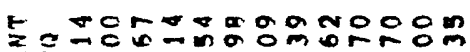

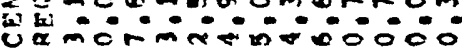
品 s

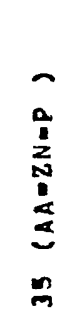

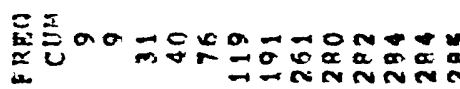

L 0.0 namminanno$\pi$

$m$

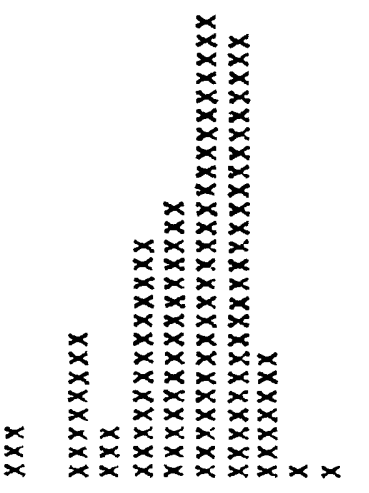

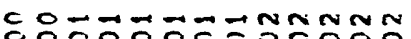

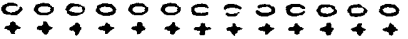

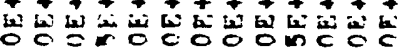

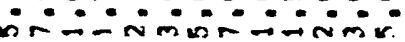

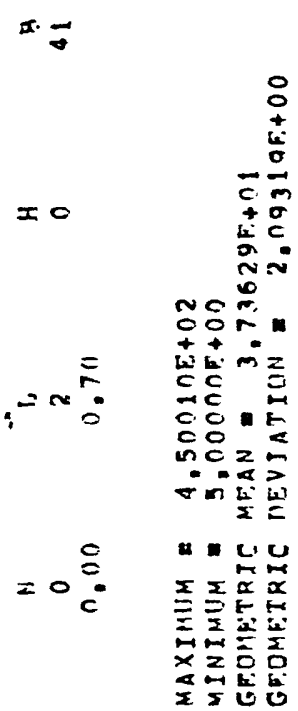




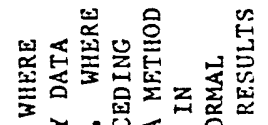

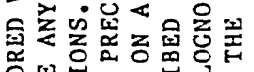

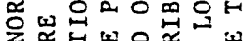

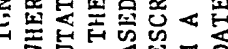

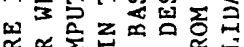

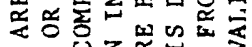

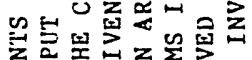

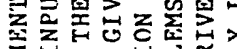

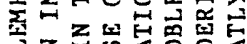

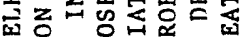

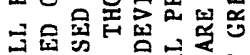

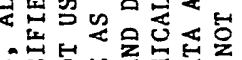

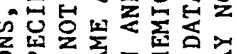

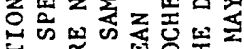

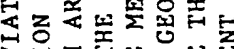

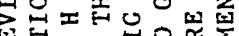

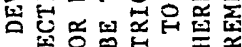

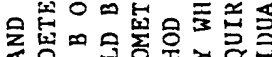

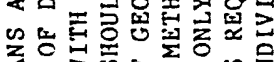

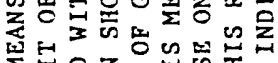

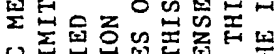

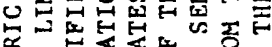

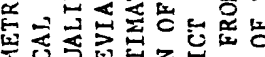

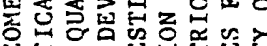

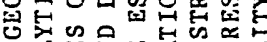

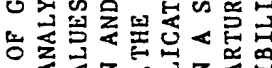

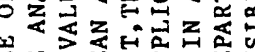

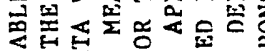

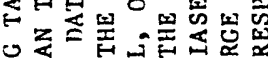

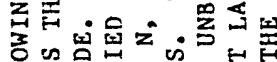

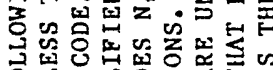

연

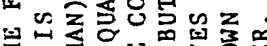

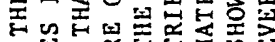

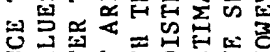

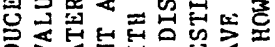

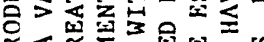

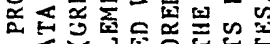

은

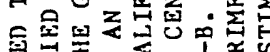

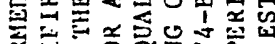

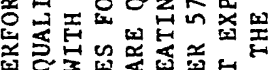

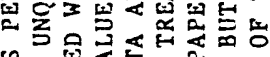

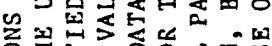

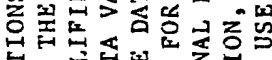

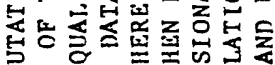

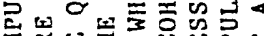

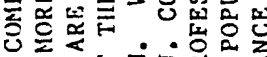

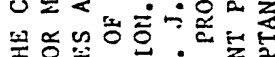

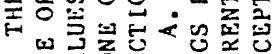

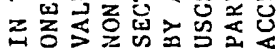

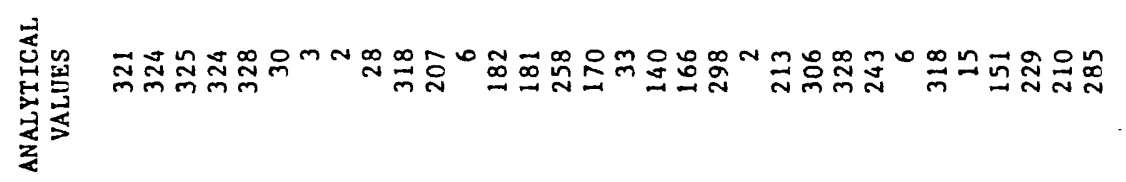

- noON00000n000000000m000000000000

is

$H 00000000000000000000000000000000$

$00000000000000000000000000000 \vec{J} \vec{F}$

I

00000000000000000000000000000000

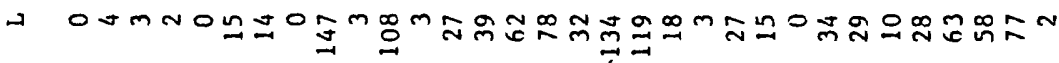

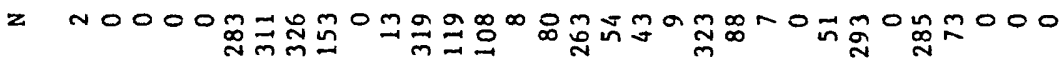

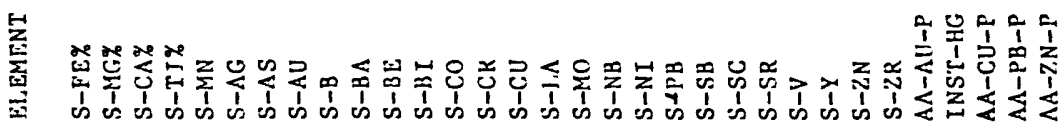




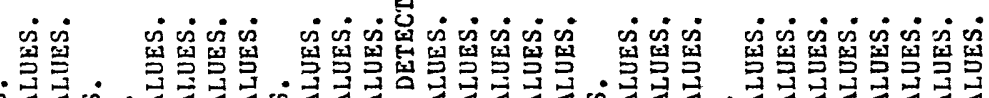

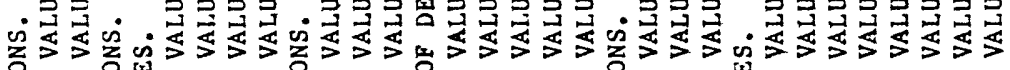

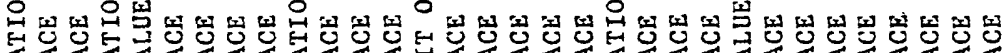

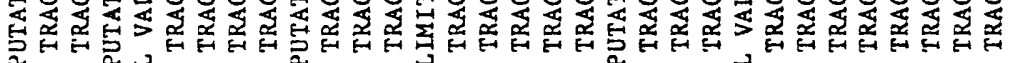

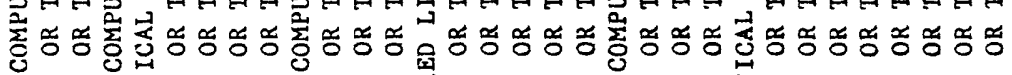

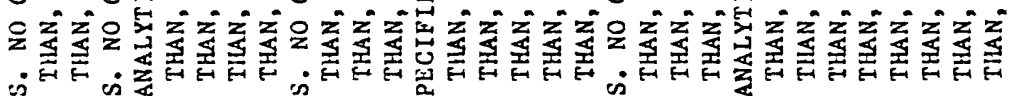
边

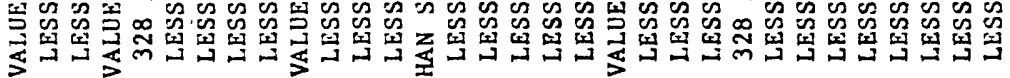

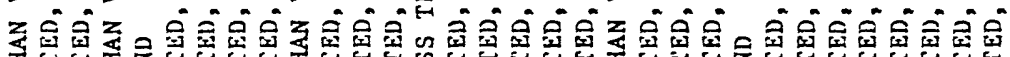

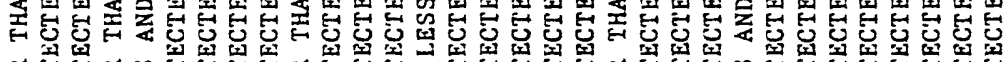

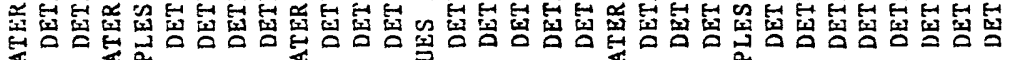

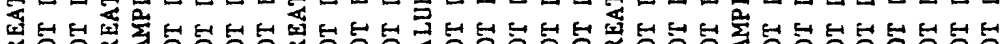

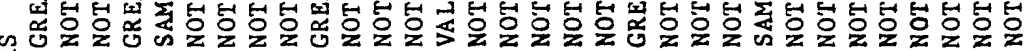
縗

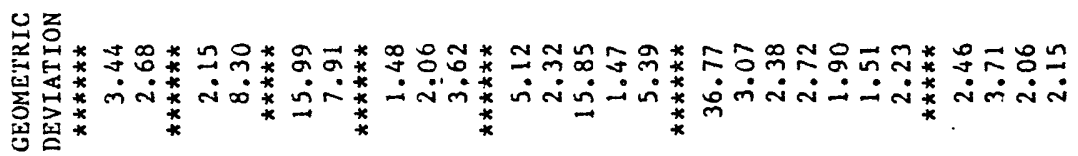

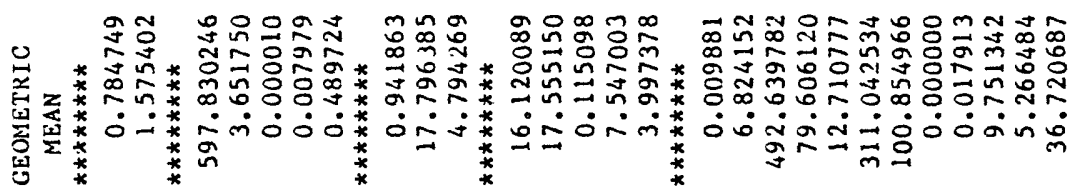

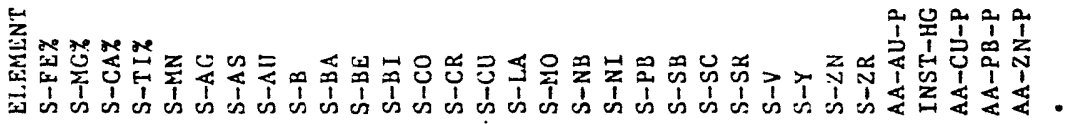



西

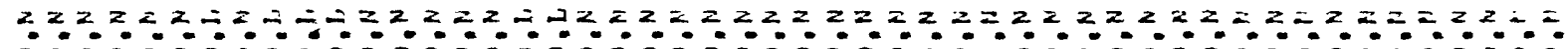
 "

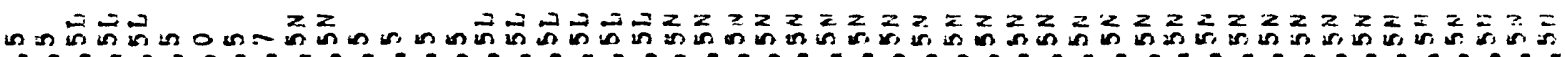

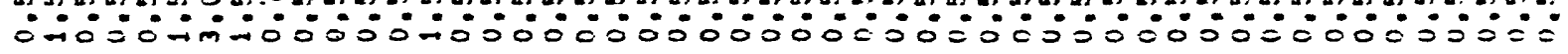

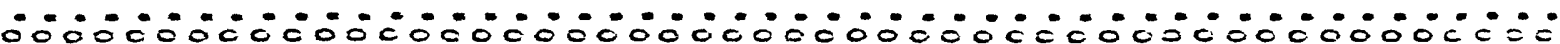

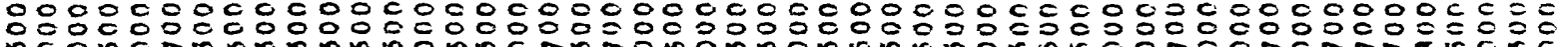

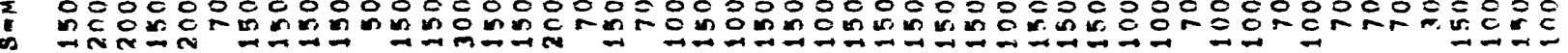

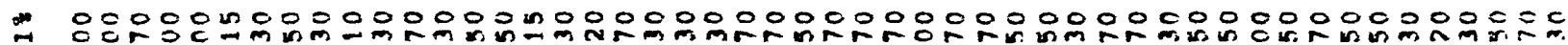
I

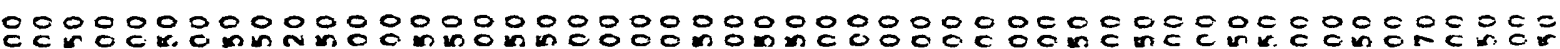
minm

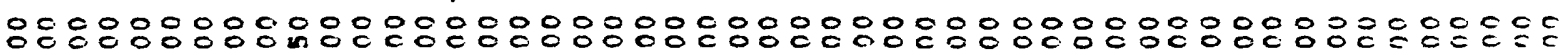

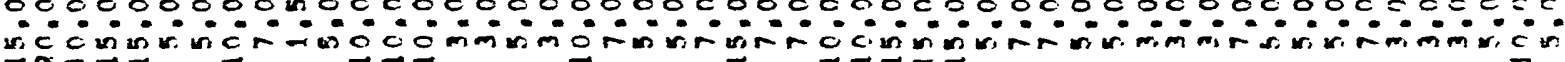

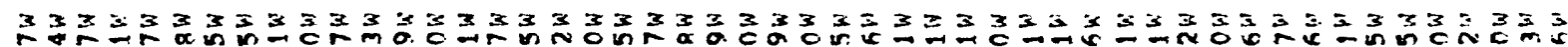

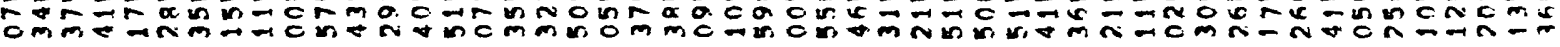

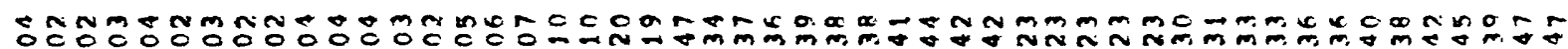

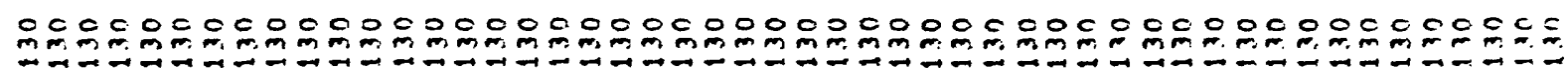

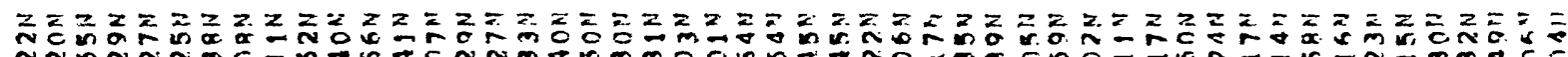

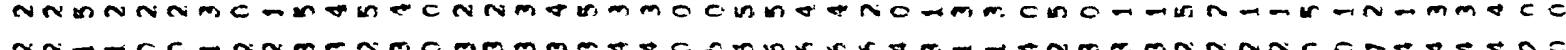

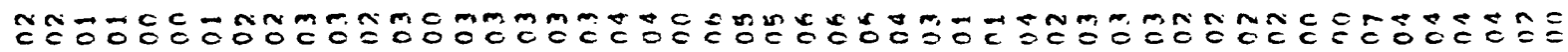

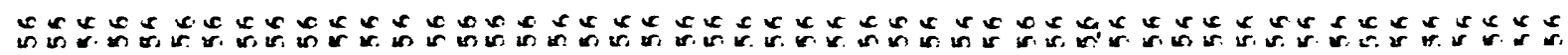




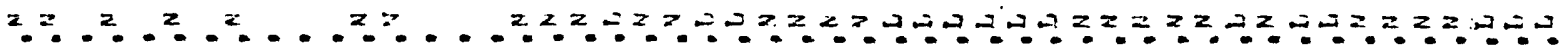
$\prod_{i}$

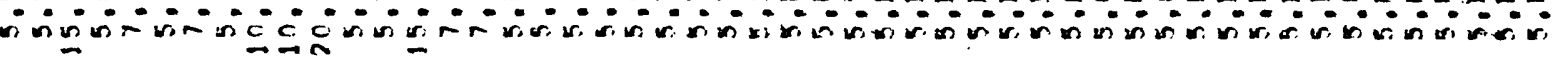

$\therefore \approx 2 \div=z$ z...............

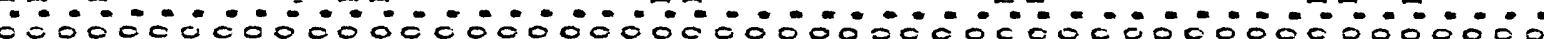

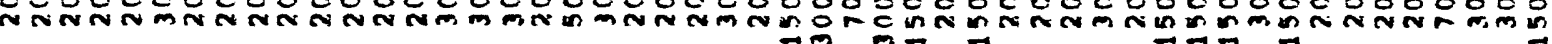

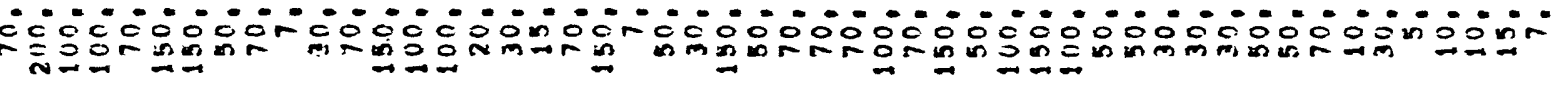

$\stackrel{0}{c}$

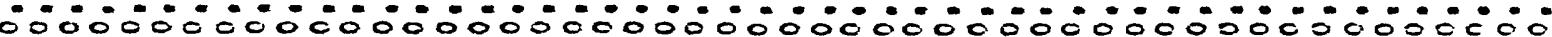

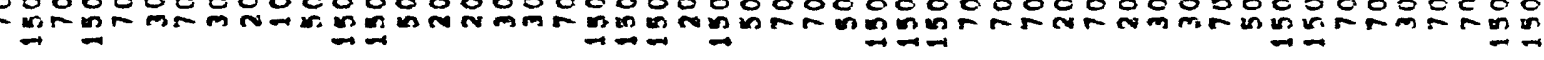

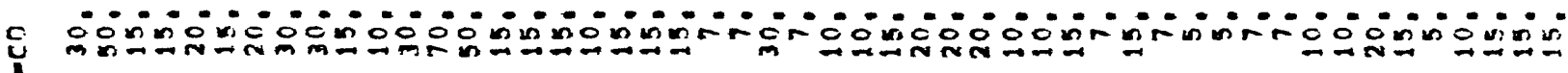

\title{
$\ln _{-\infty}=0$
}

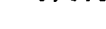

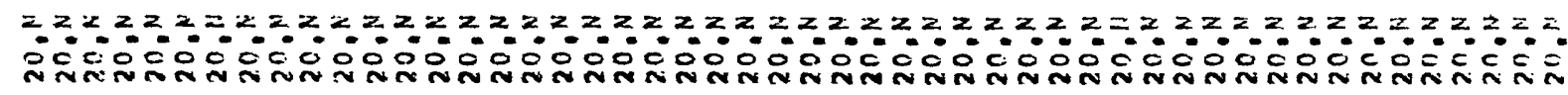

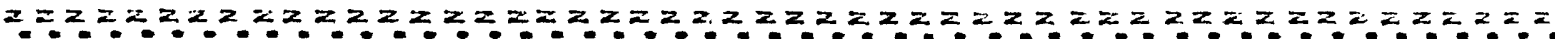

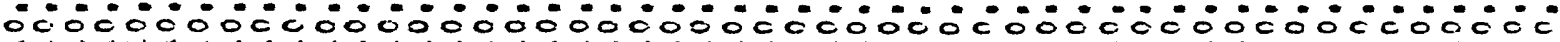

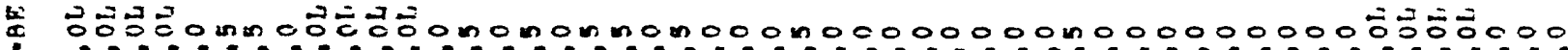

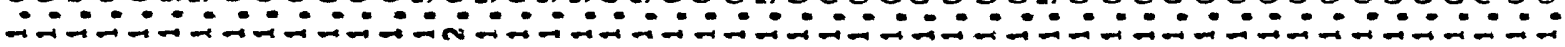

-

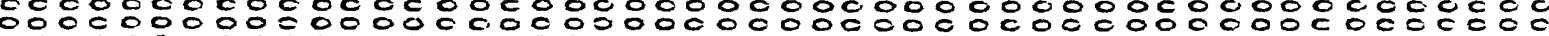

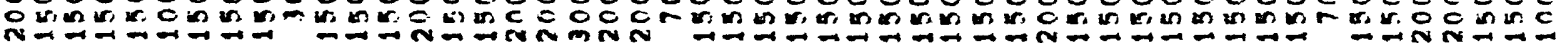

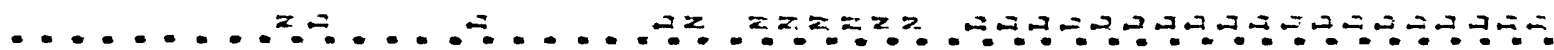

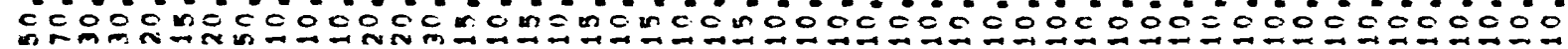

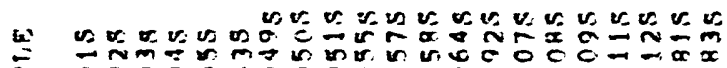

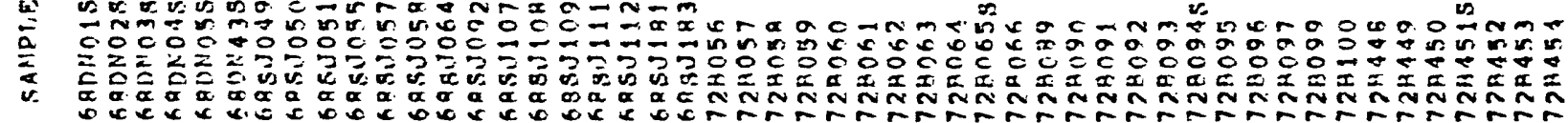




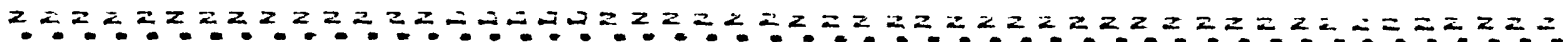

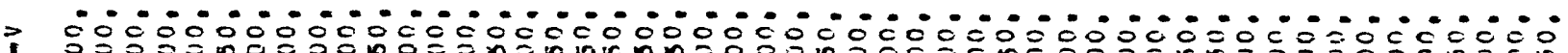

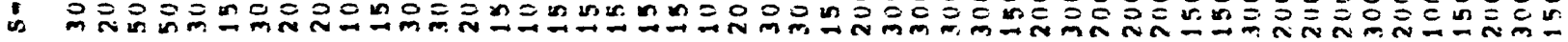

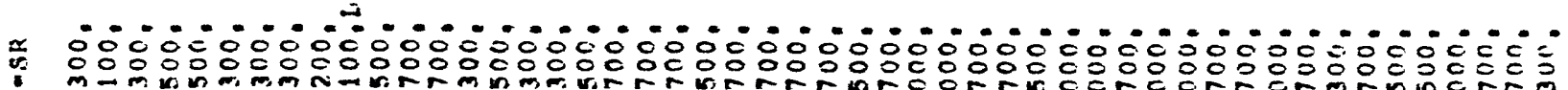

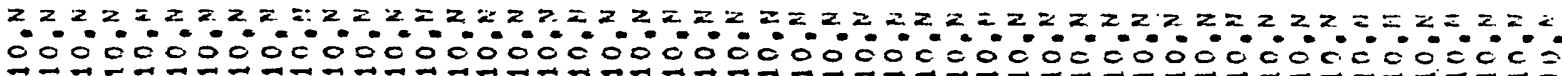
:

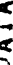

:

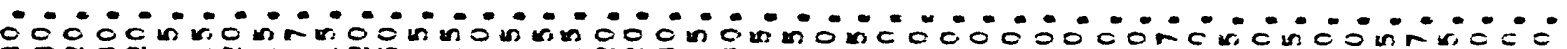
$\infty$

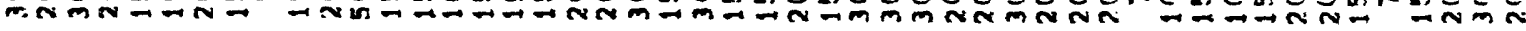

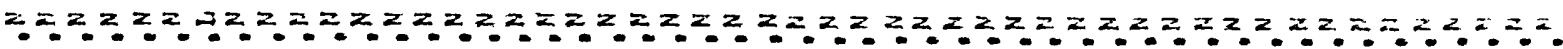

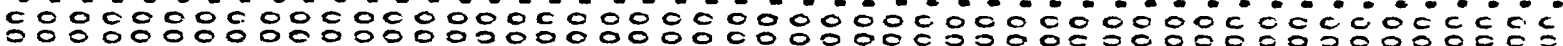

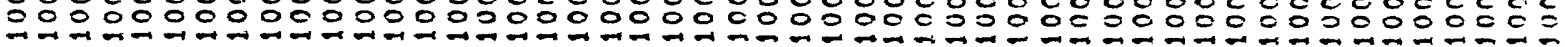

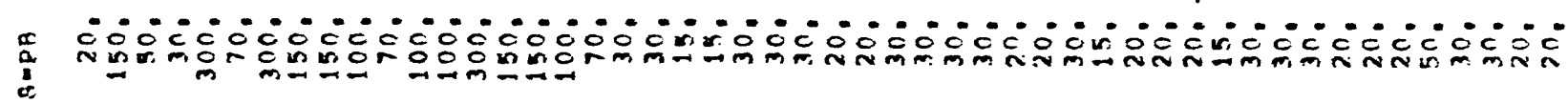

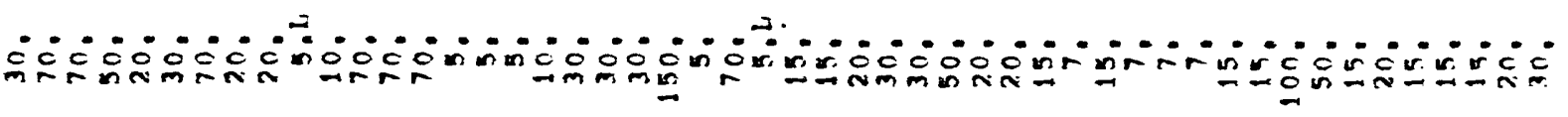

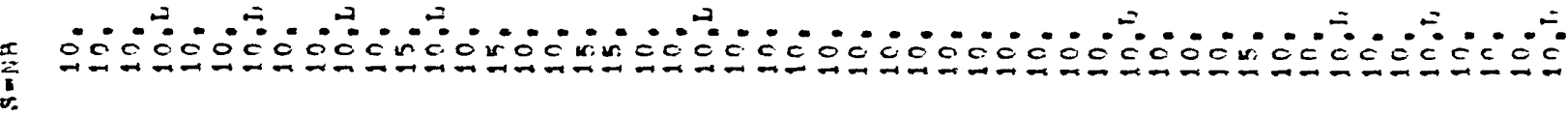




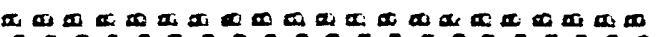

00000000000000000000

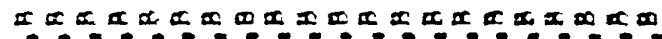

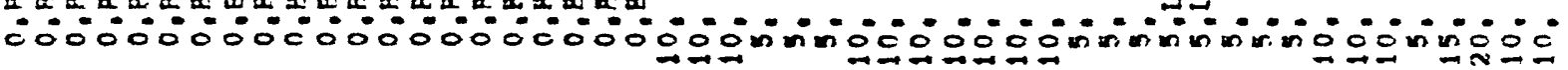

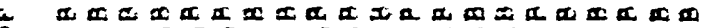

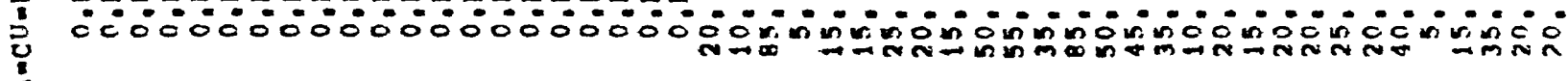

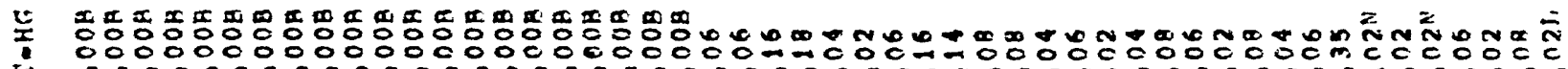
0000000000000000000000000000000000000000000000

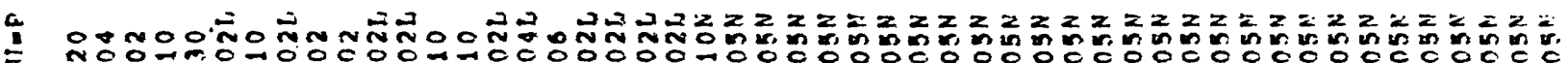
$\therefore 000000000000000000000000000000000000000000000000$

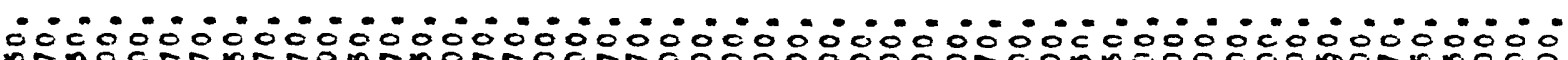

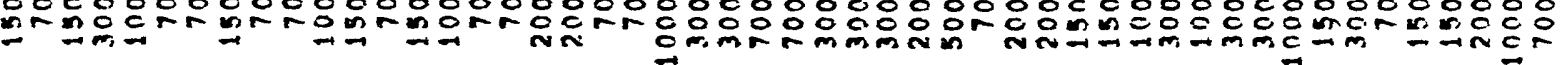

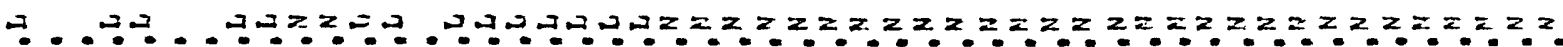

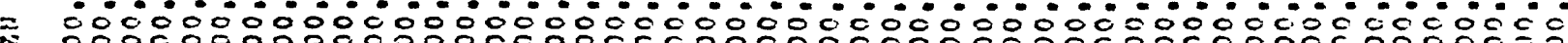

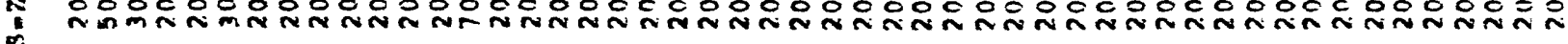

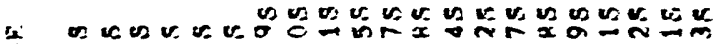

20

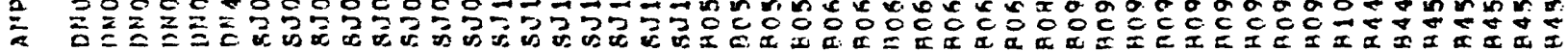

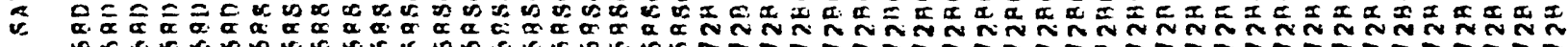




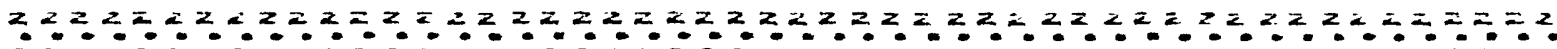

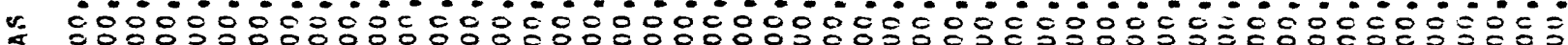

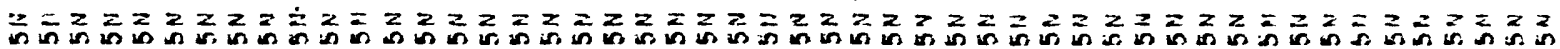

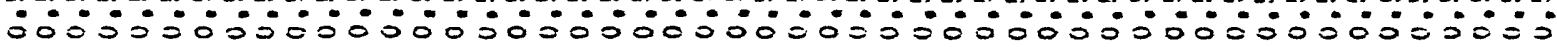

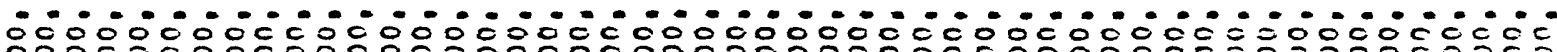
r

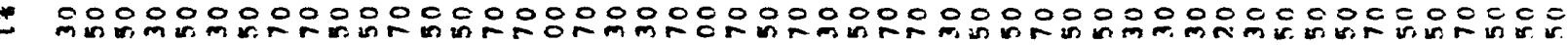

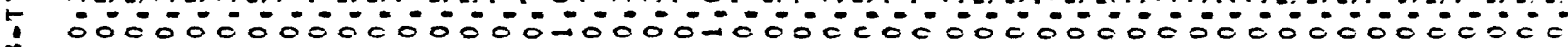

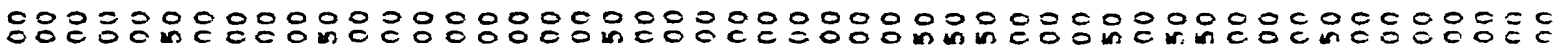

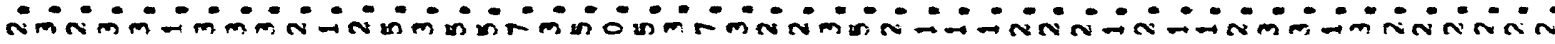

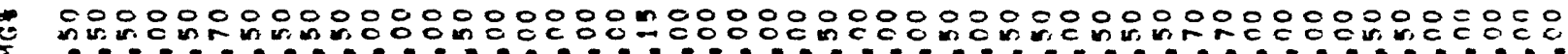

I
\end{abstract}

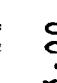

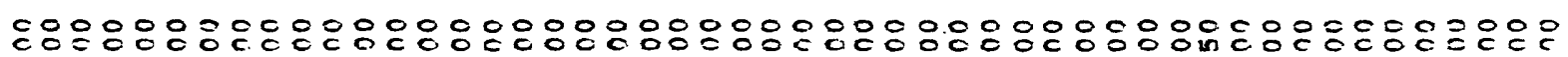
nuvinmuninumm

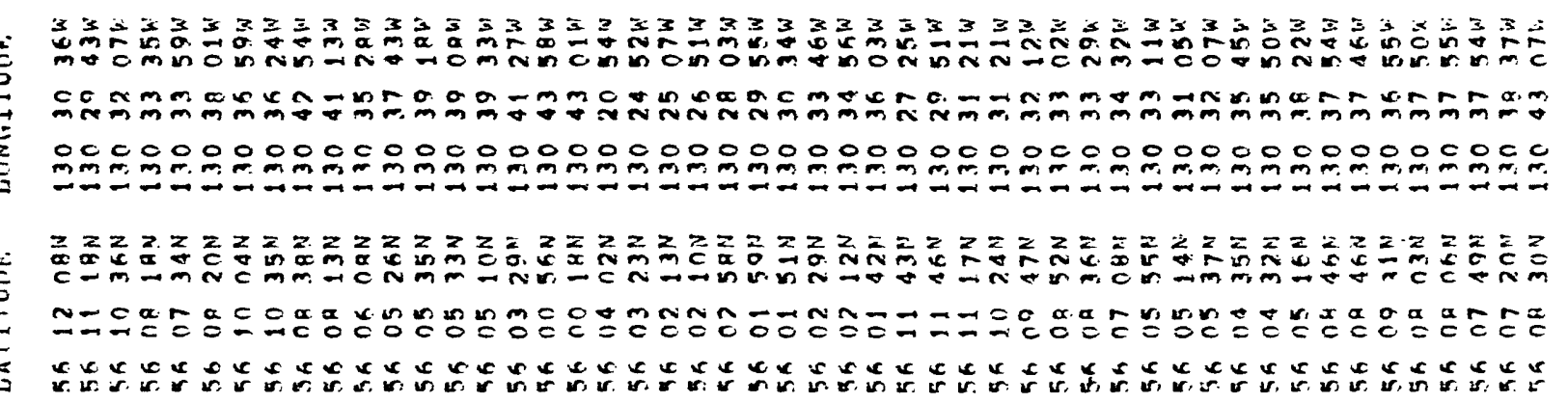

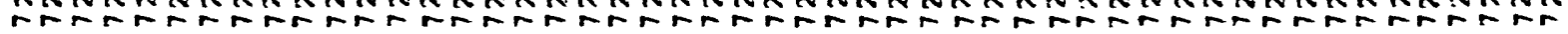




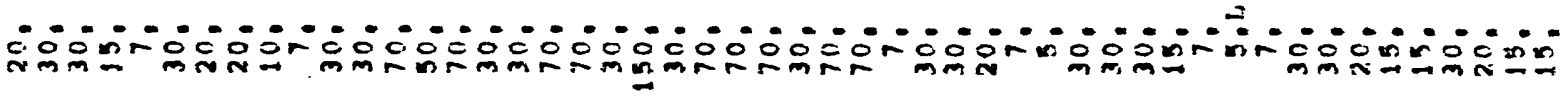

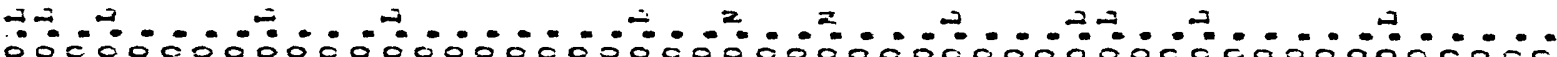

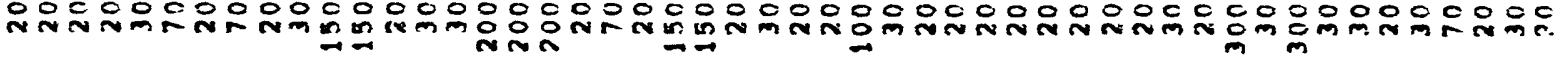

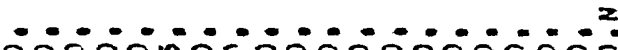

$z$

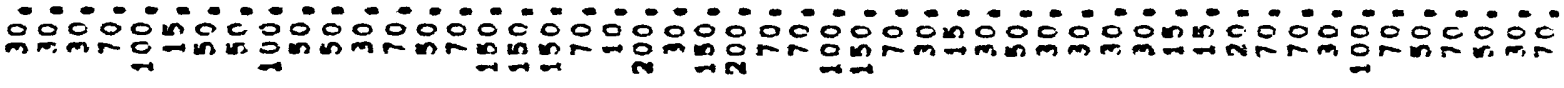

\author{
$\bullet$
}

:

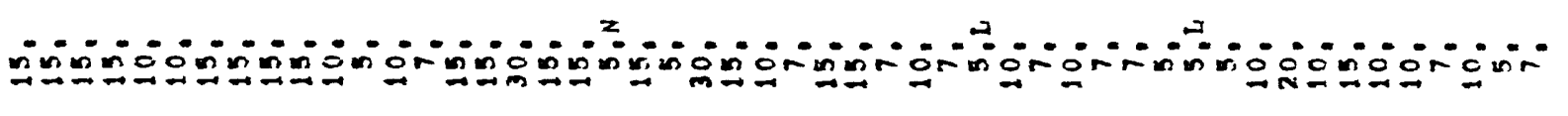

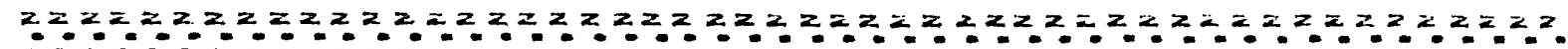

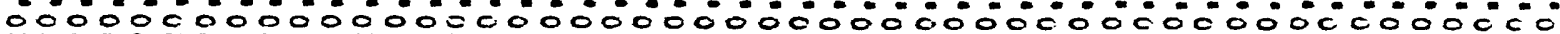
nen

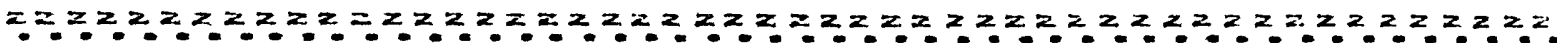
00000000000000000000000000000000000000000000000

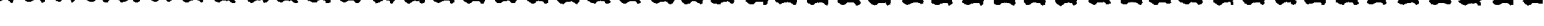

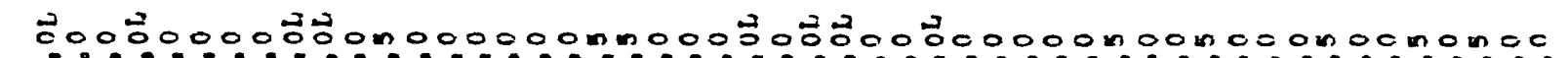

"

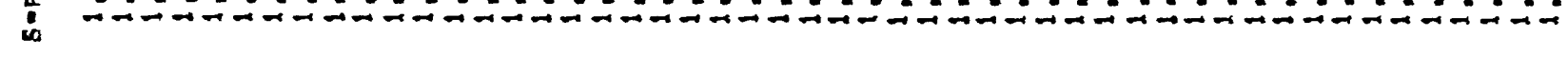

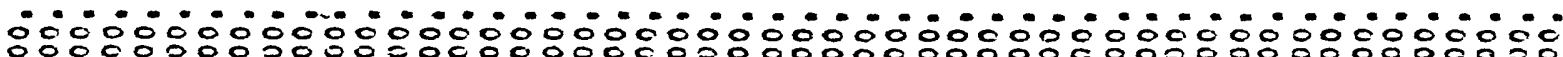

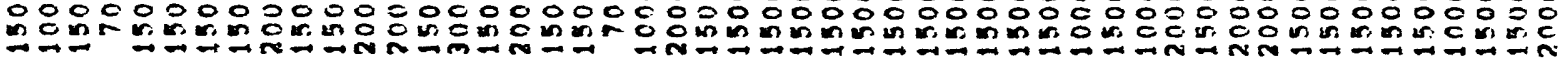

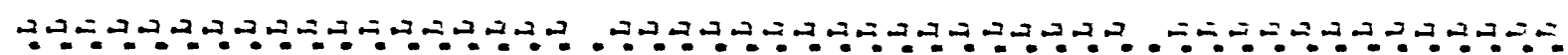
000000000000000000000000000000000000000000

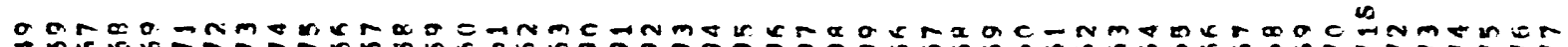

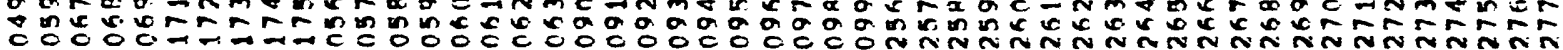

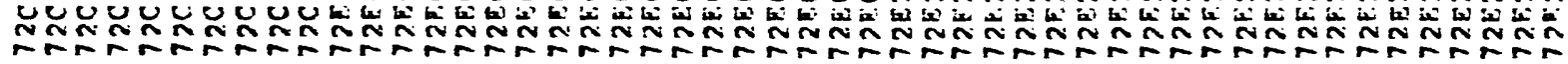




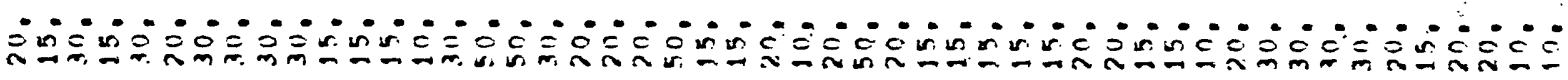

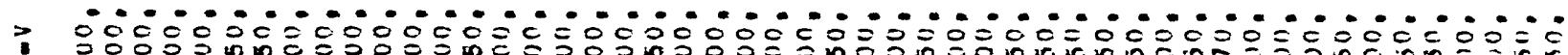

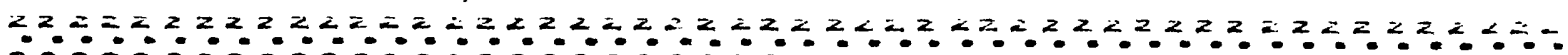
o

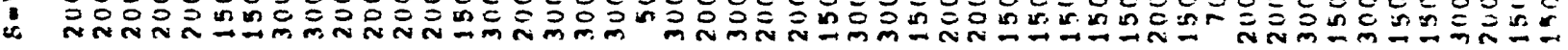

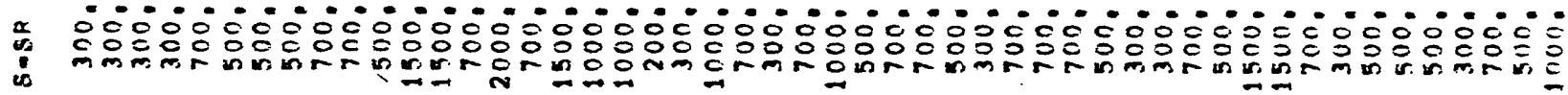

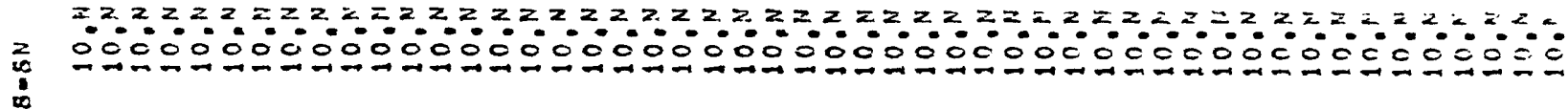

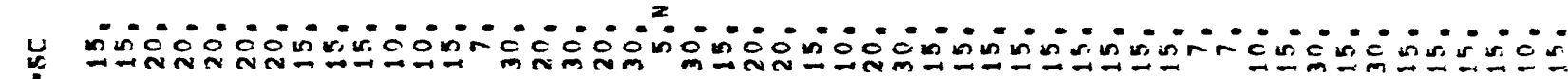

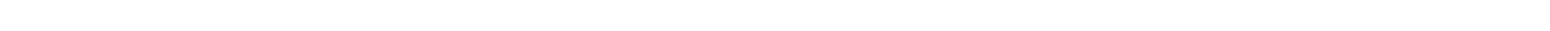

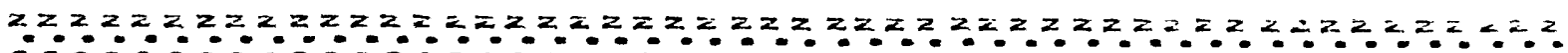

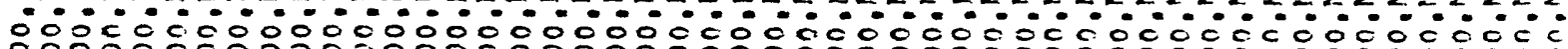

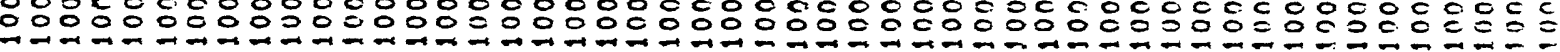

줄

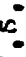
के$$
-
$$

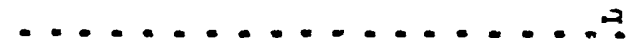

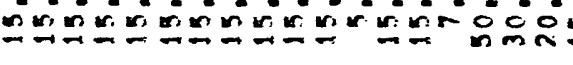

$\therefore 0$

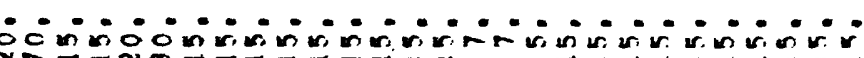
(n)

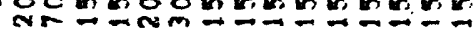

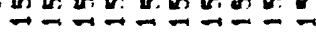

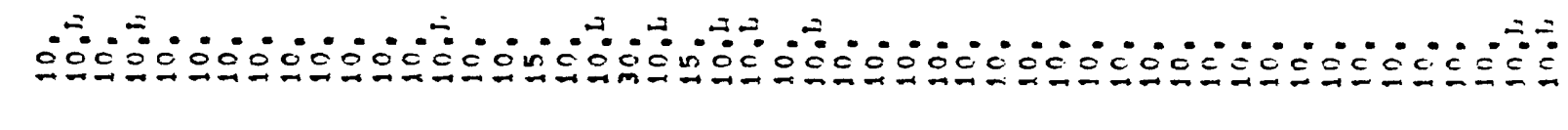

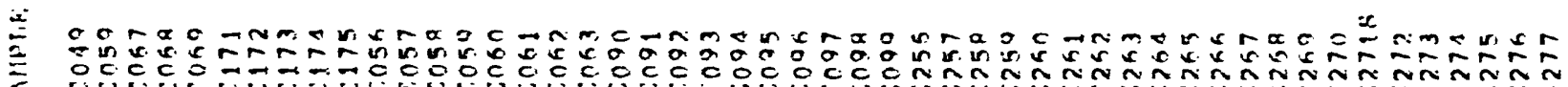

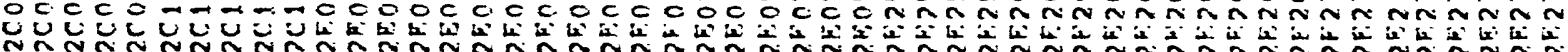
Noñ 
onóñn

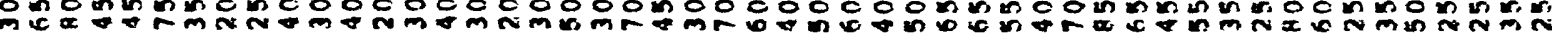

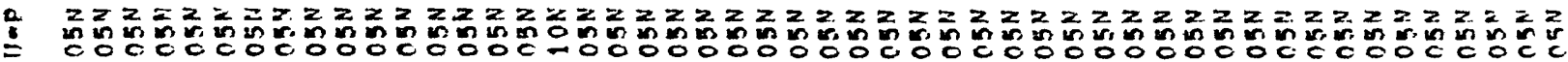
ó00000

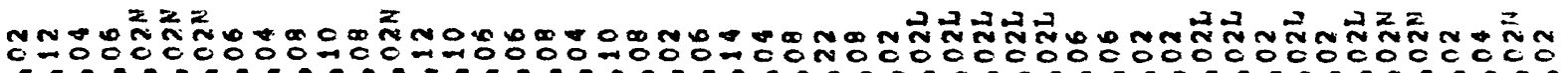

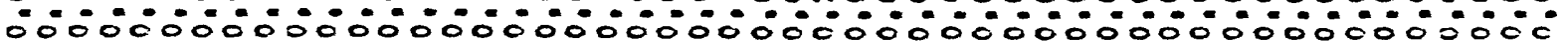

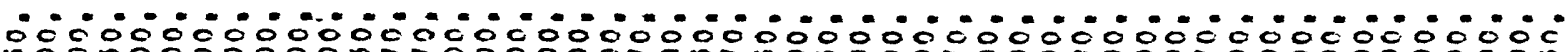

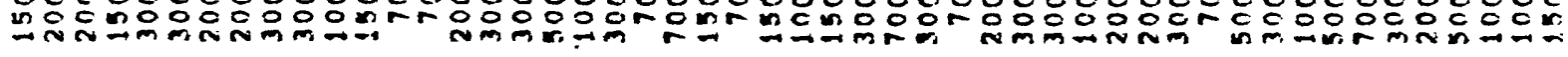

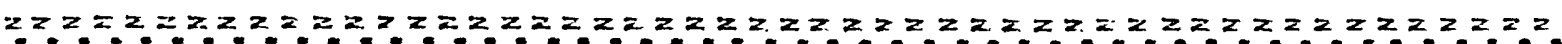

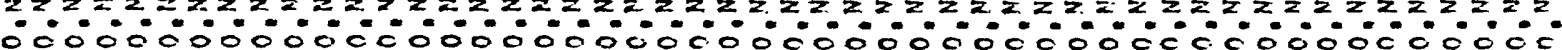
웅에 
$z=2 z=2$

$2 z=2 z=2 z 2 z=2 z z=20$

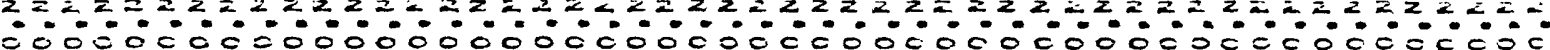

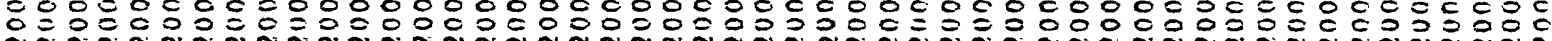

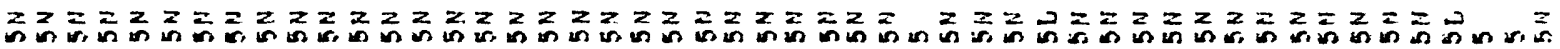

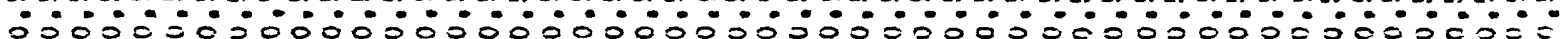

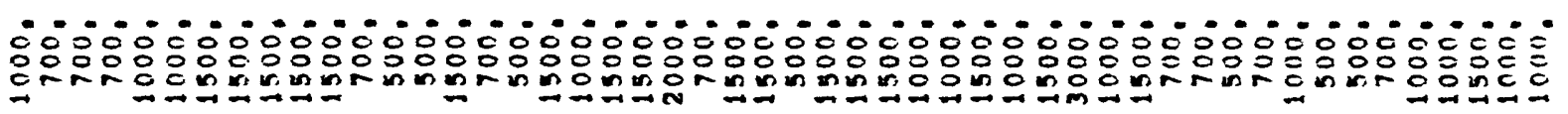

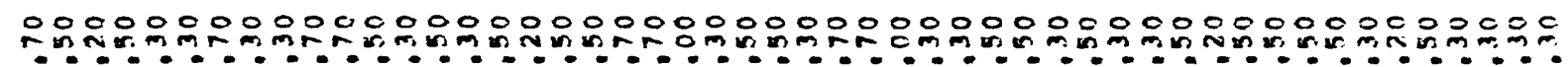
$0000000000000000000-10000-10000000000000000$
\end{abstract}

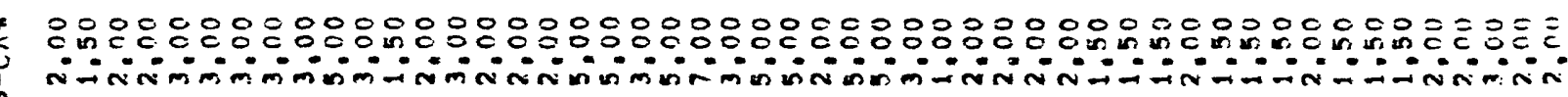

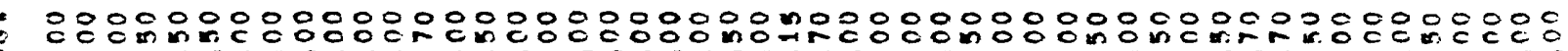

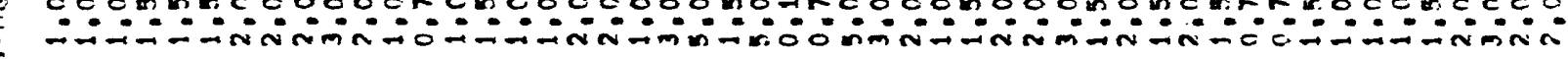

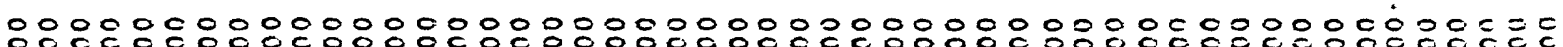

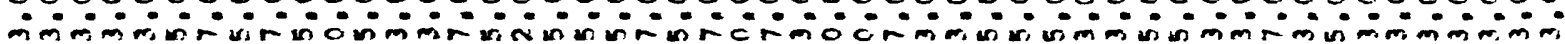

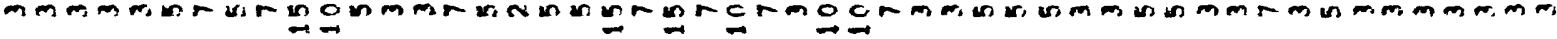

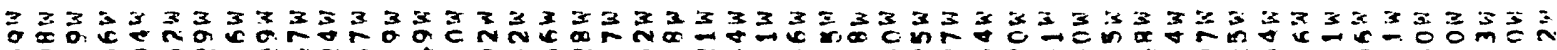

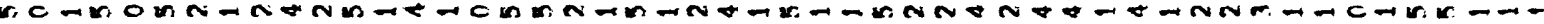

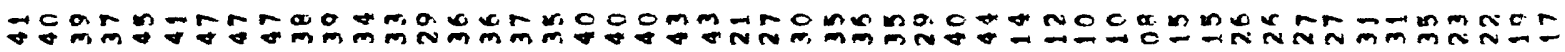
$0=000000000000000000000000000000-\vec{m}---\cdots 000000000=0$

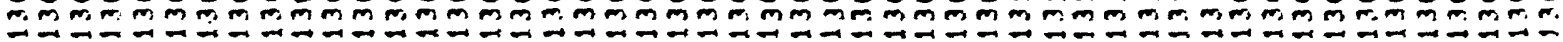

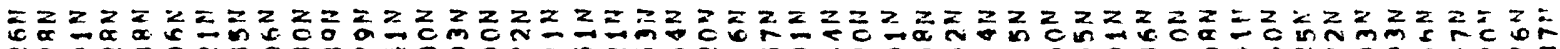

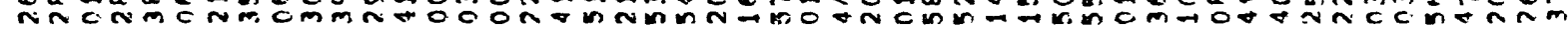

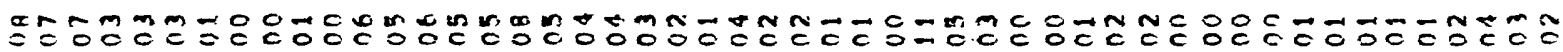

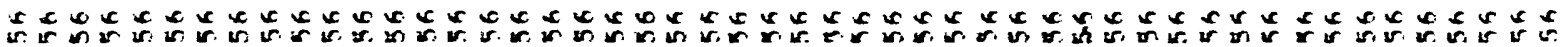

L $\quad$ a

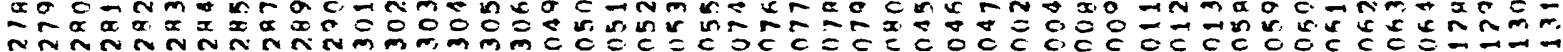
N N N N N

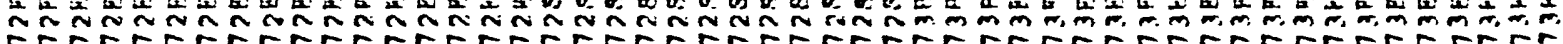




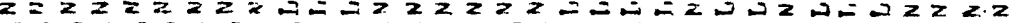
$=2 z 2 z z 2 z z 2 z z=2$ $\stackrel{5}{i}$

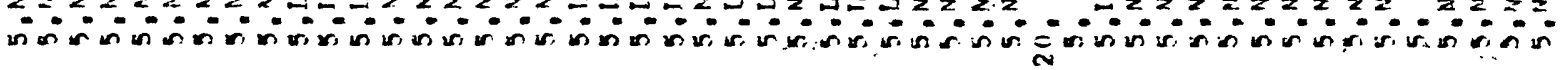

$\stackrel{2}{\stackrel{2}{2}}$

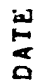

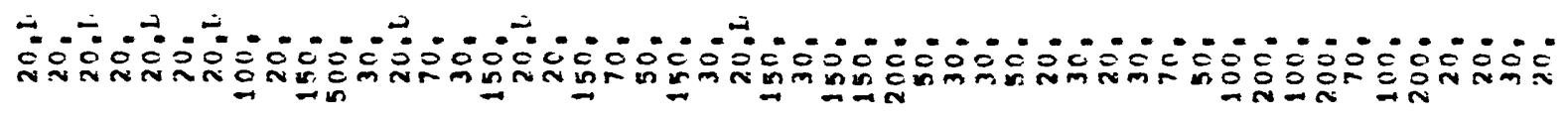

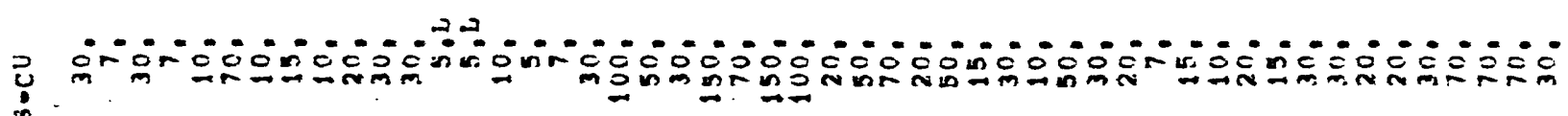

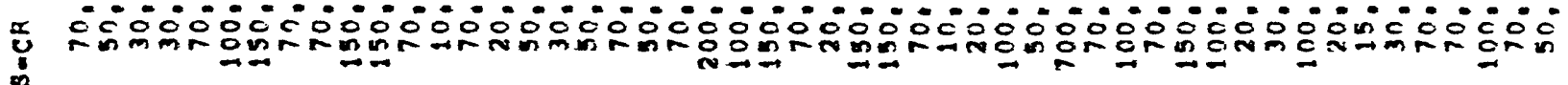

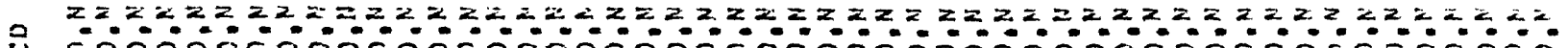

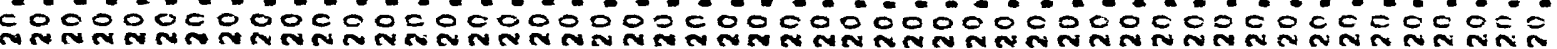
$\mathbf{m}$

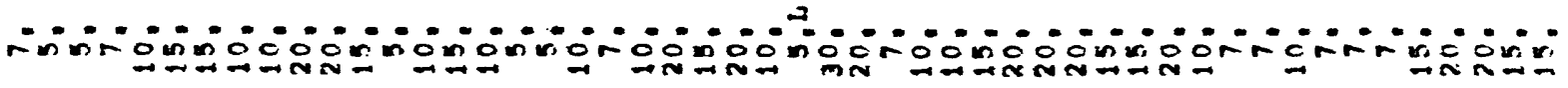

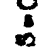
(2)

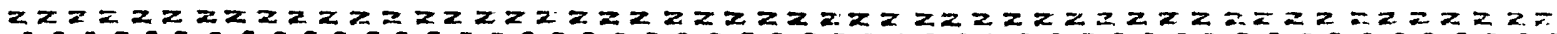

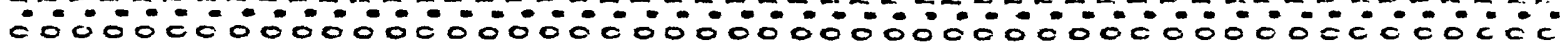

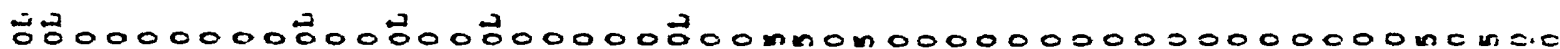
๘

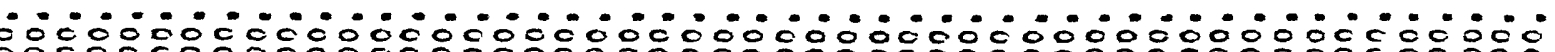
00000000300000000000000000000000008000005000000 - 푸은

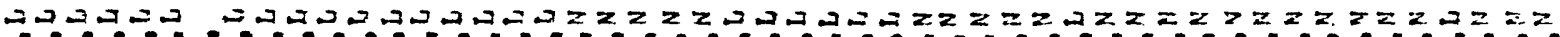
00000000000000000000000000000000000000 


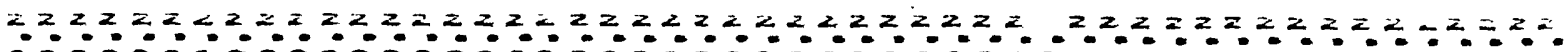

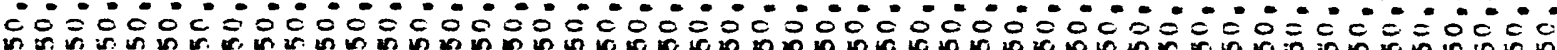

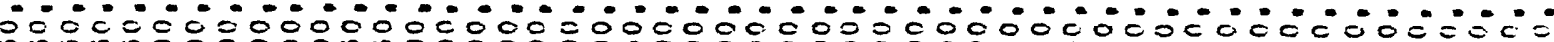
m는

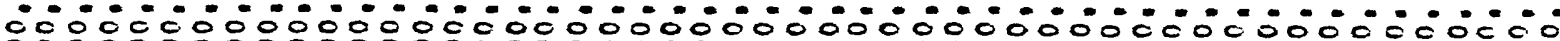

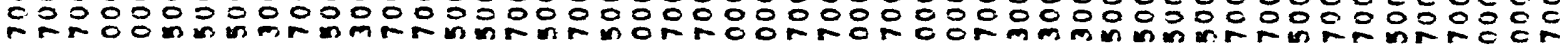

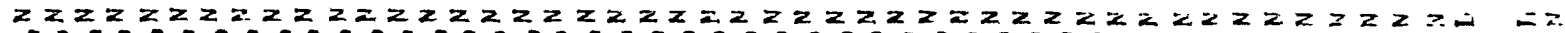
000000000000000000000000000000000000000000000000

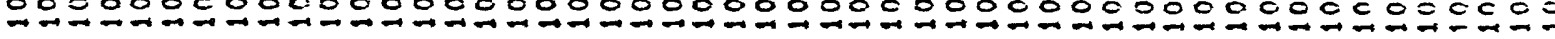

ثñ

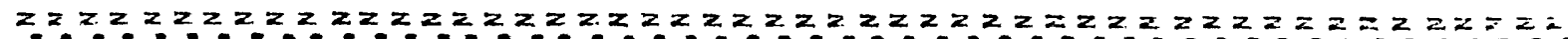

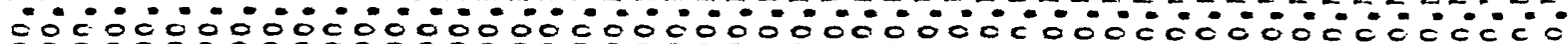

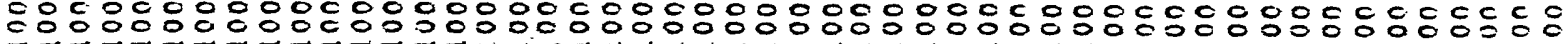

-

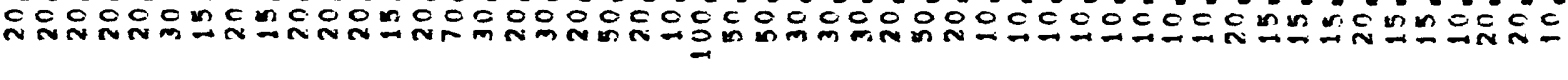

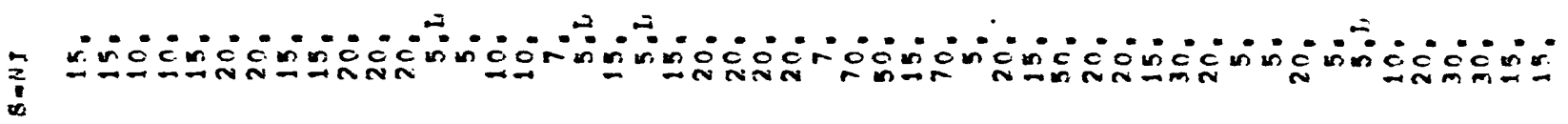
50000000000000000000000000000
$\vdots$ 


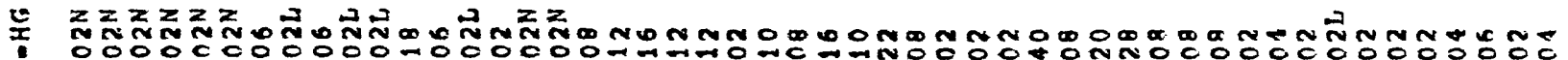

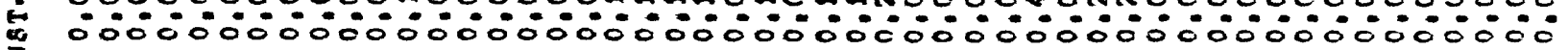

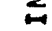

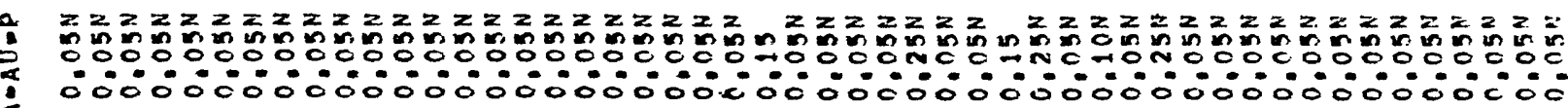

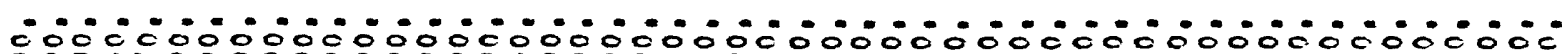

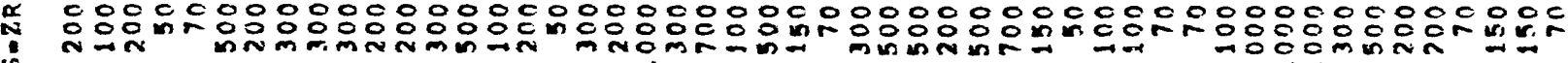
Natin

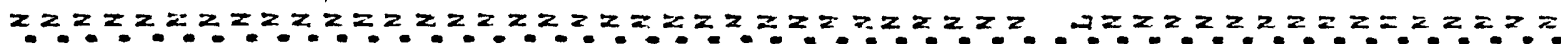
$\therefore 0000000000000000000000000000000000000000000000000$

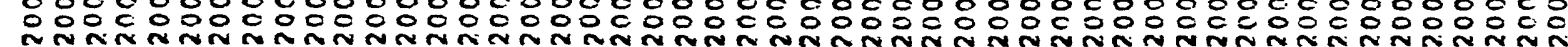




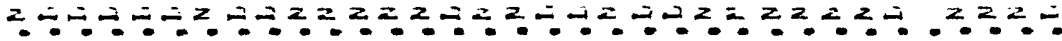

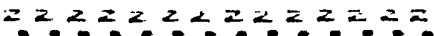

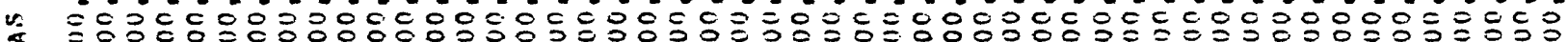

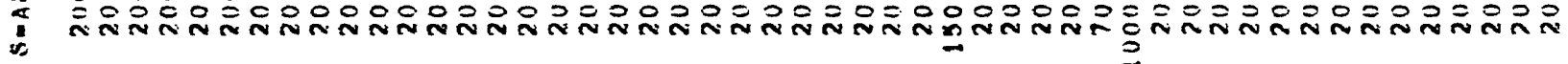

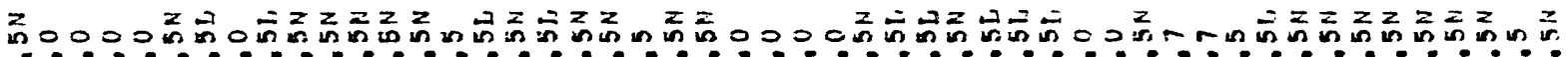

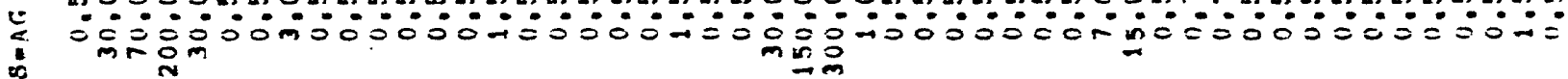

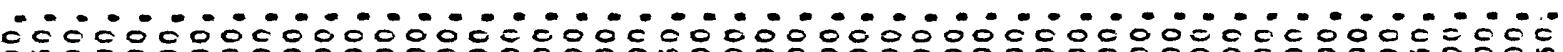

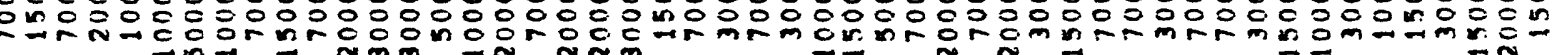
(2)

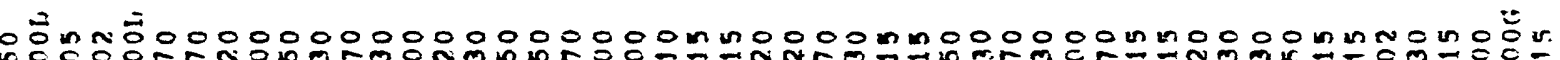
có00 (1)

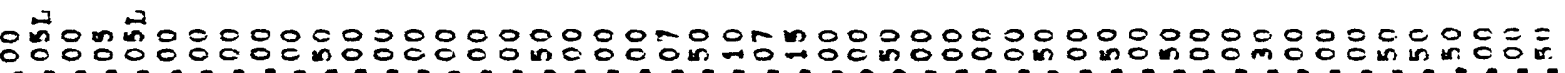

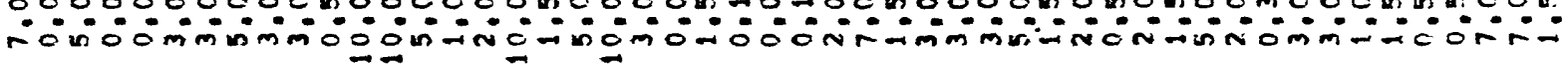

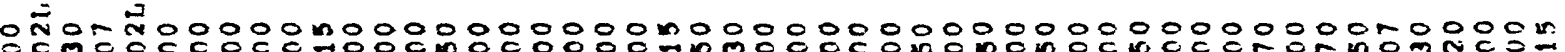

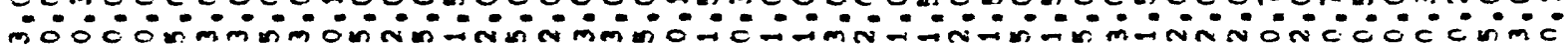

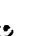

$$
\text { 依 }
$$

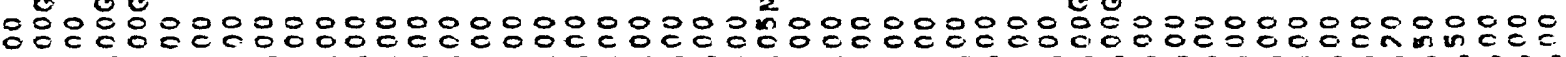

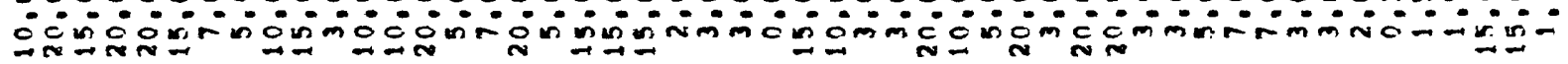

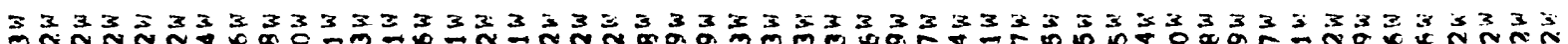

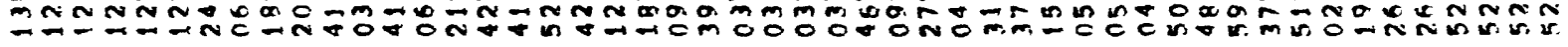

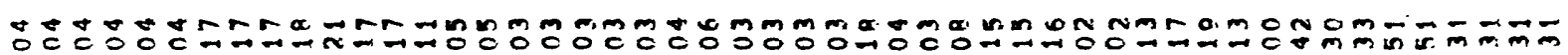

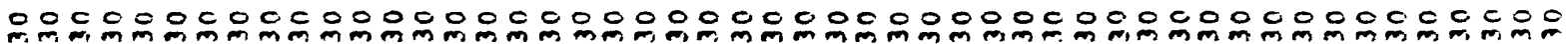

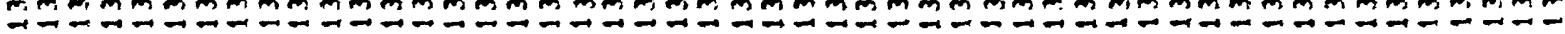

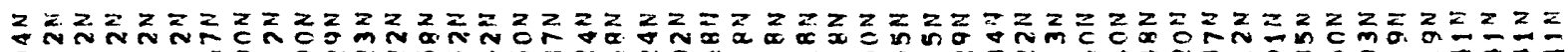

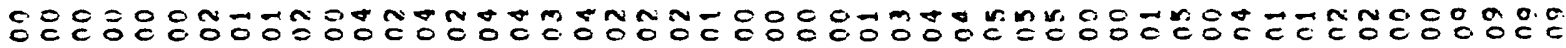

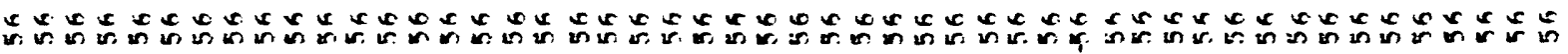


?

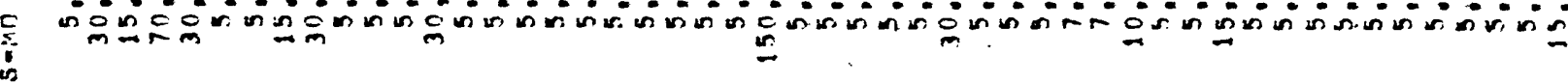

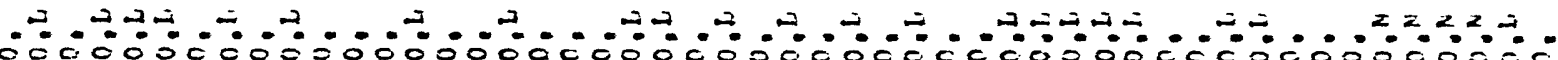

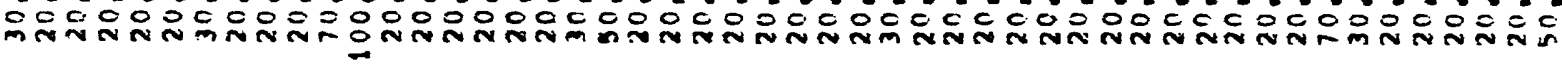

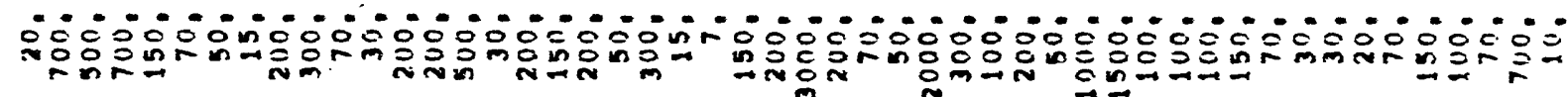

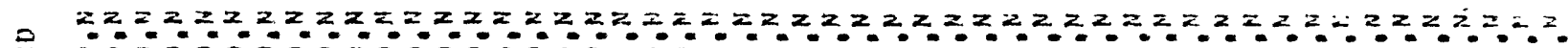

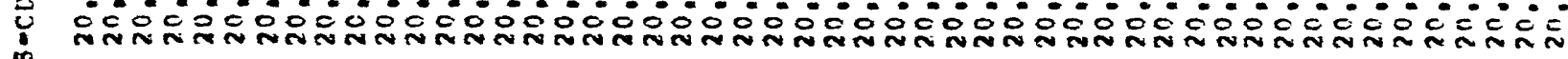
$\infty$

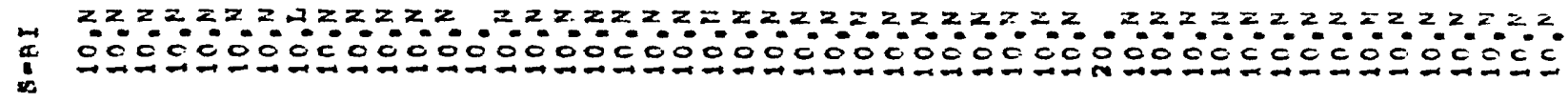

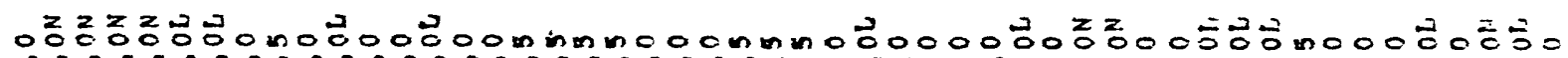
r $\infty$

2.

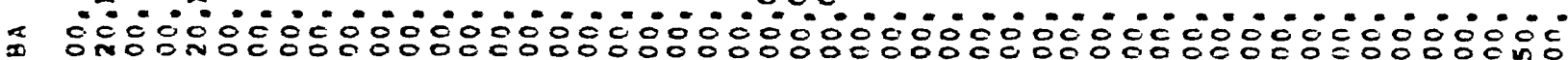
당

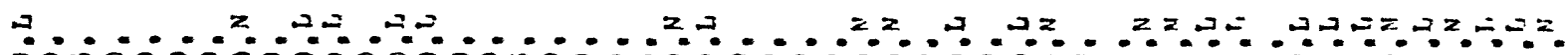

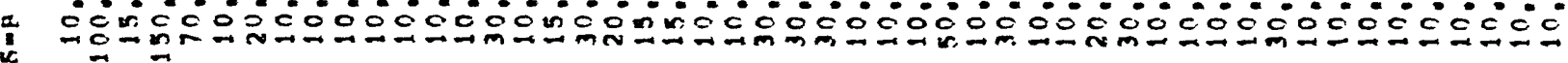




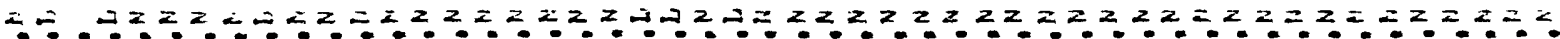

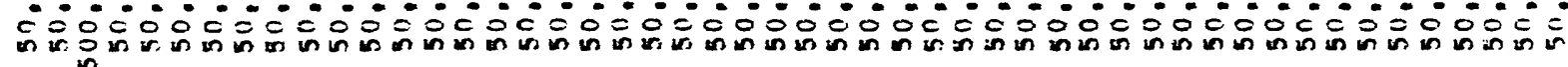

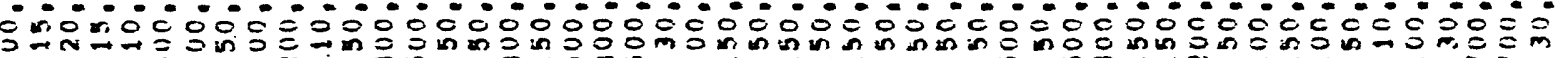

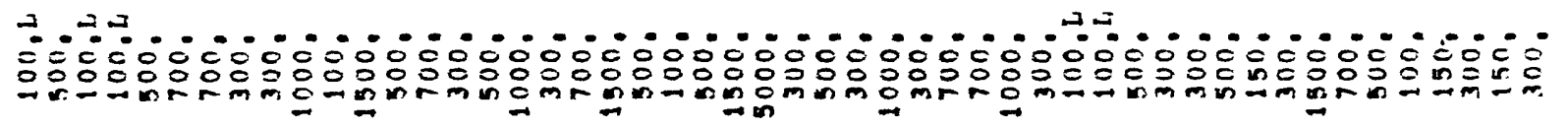

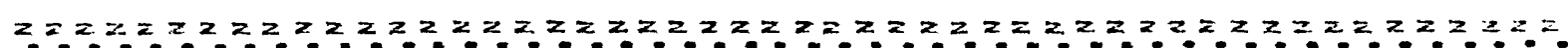

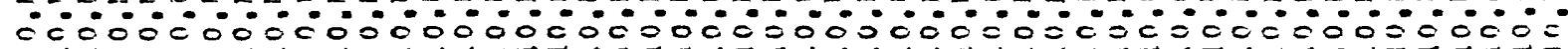

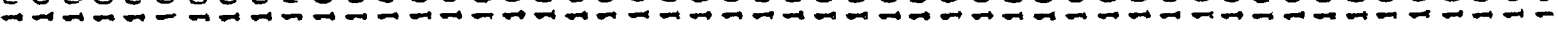

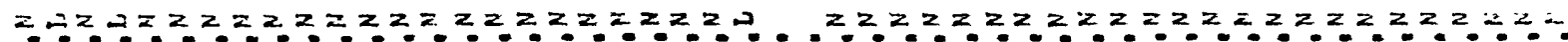

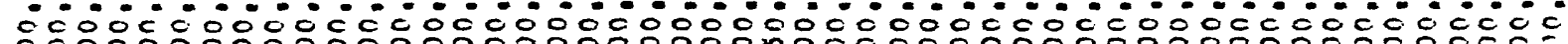

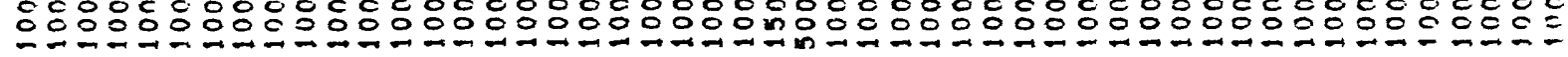

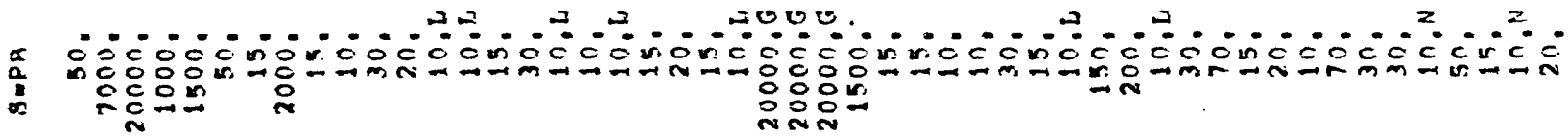

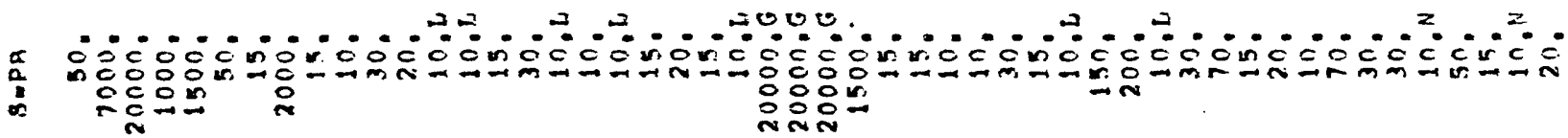
nón

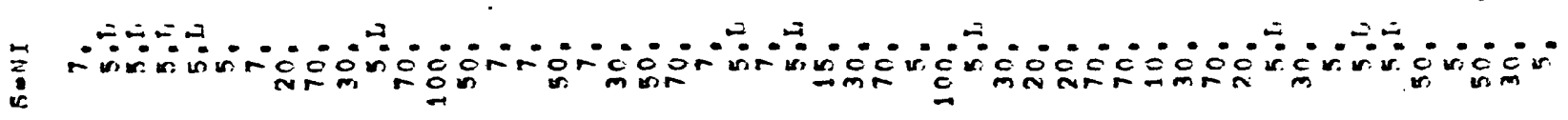
2
2 


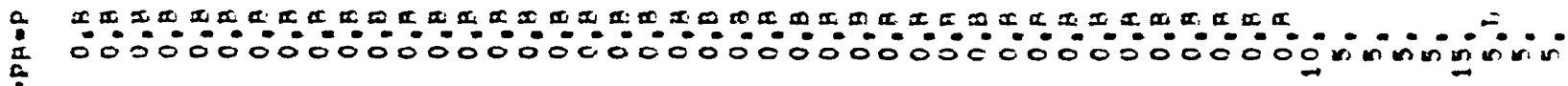

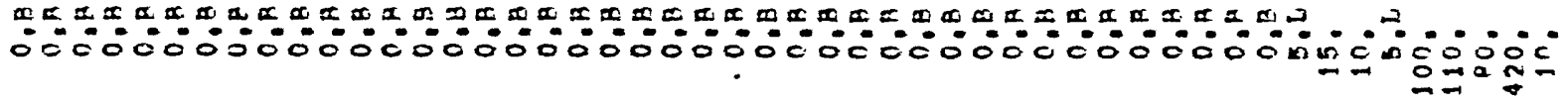

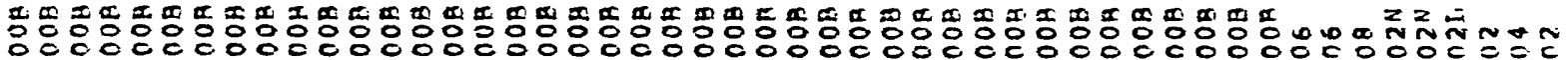
$\therefore 0000000000000000000000000000000000000000000$

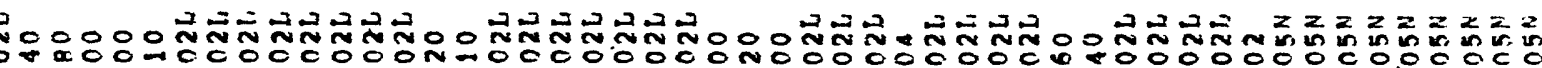
i-

7 .

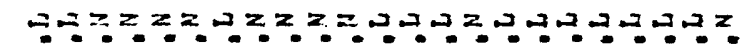

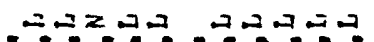
$2 z=2 z 2=2 z=2$

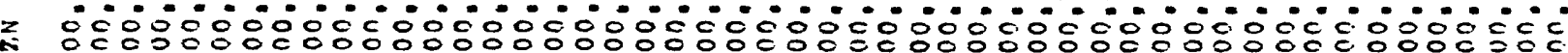
:

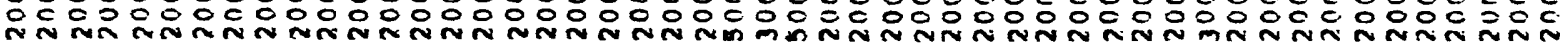




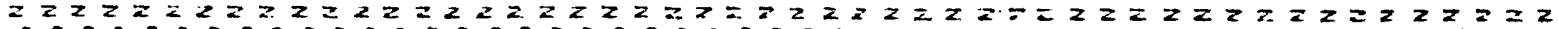

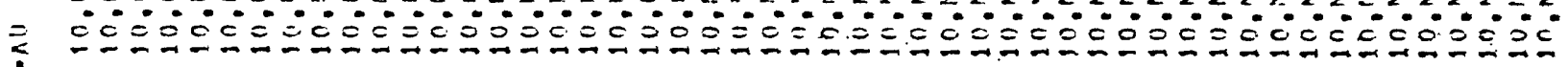

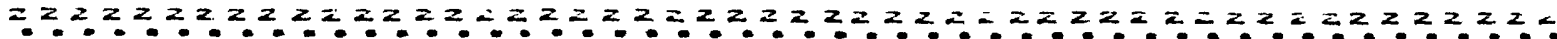

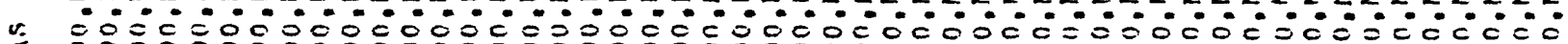

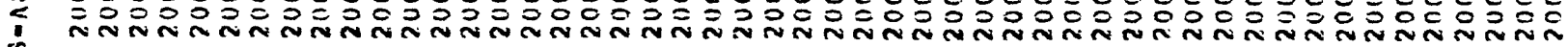

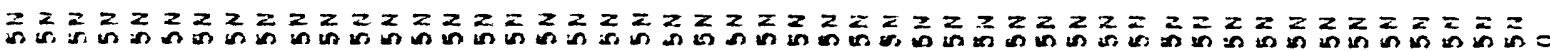
\&

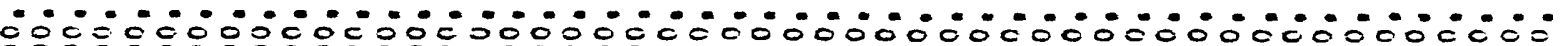

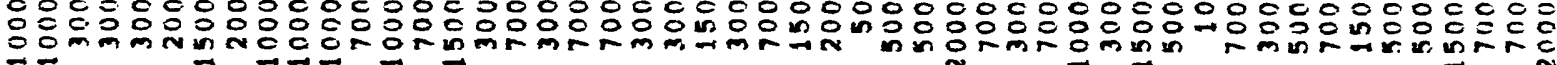

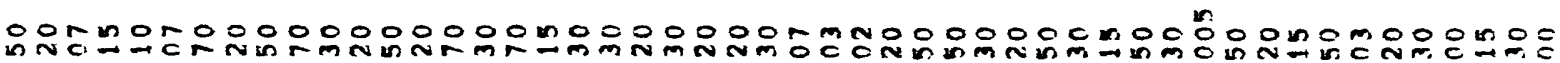
$00000000000000000000000000000000000000000000-100$

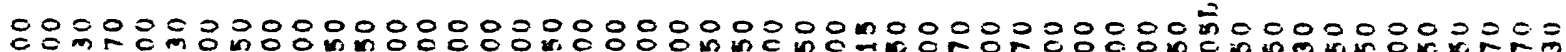
máo-

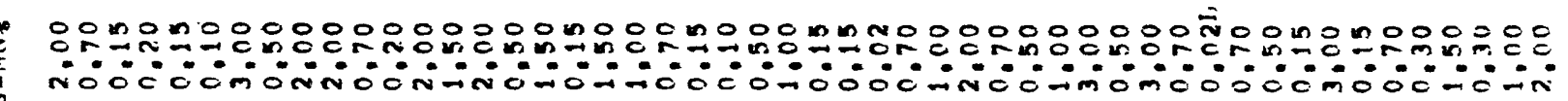

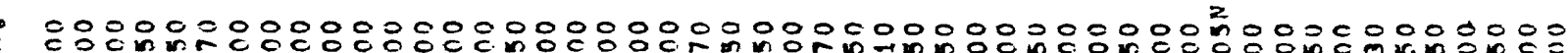

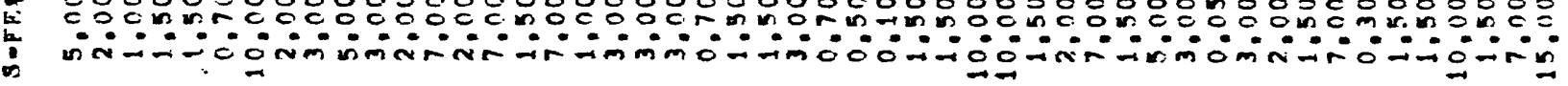
$\rightarrow+4$

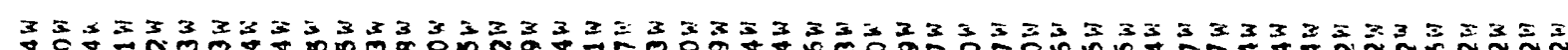

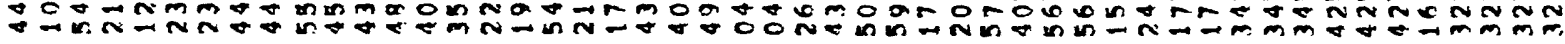

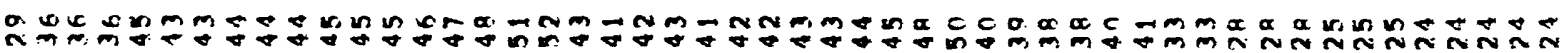

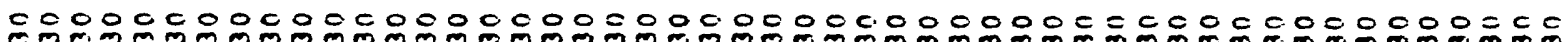

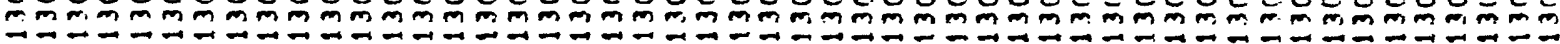

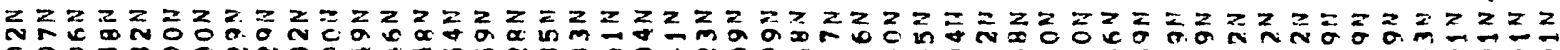

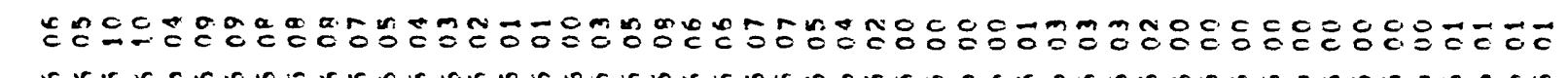

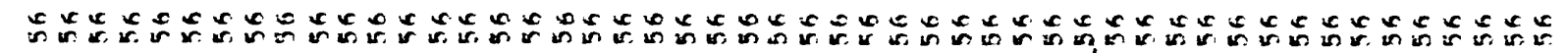

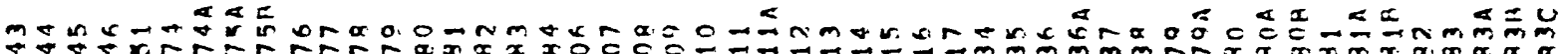

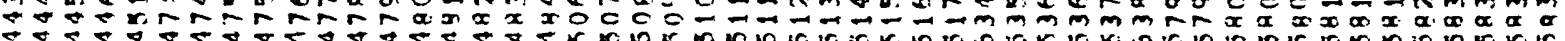

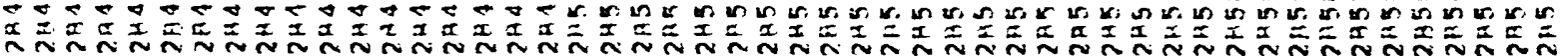

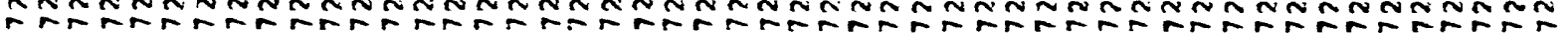




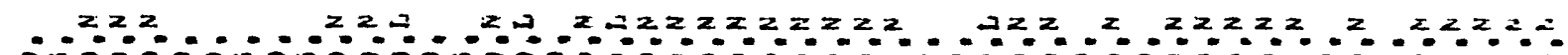

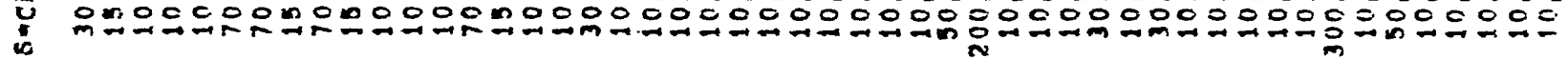

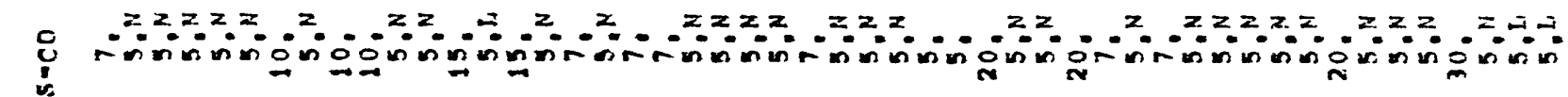

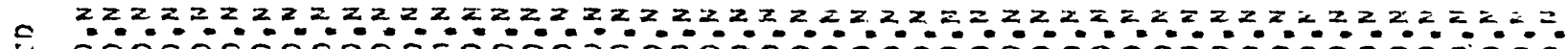

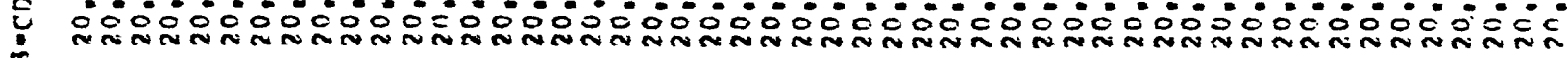

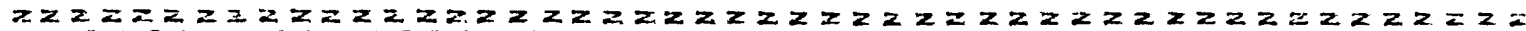

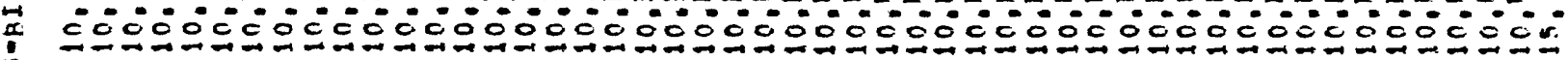

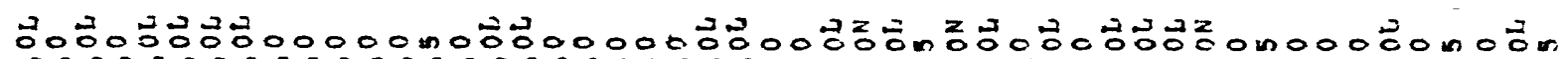

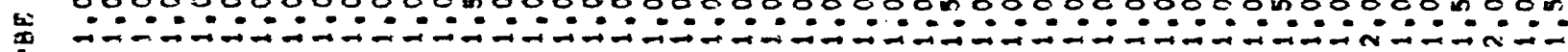
is $\supset$

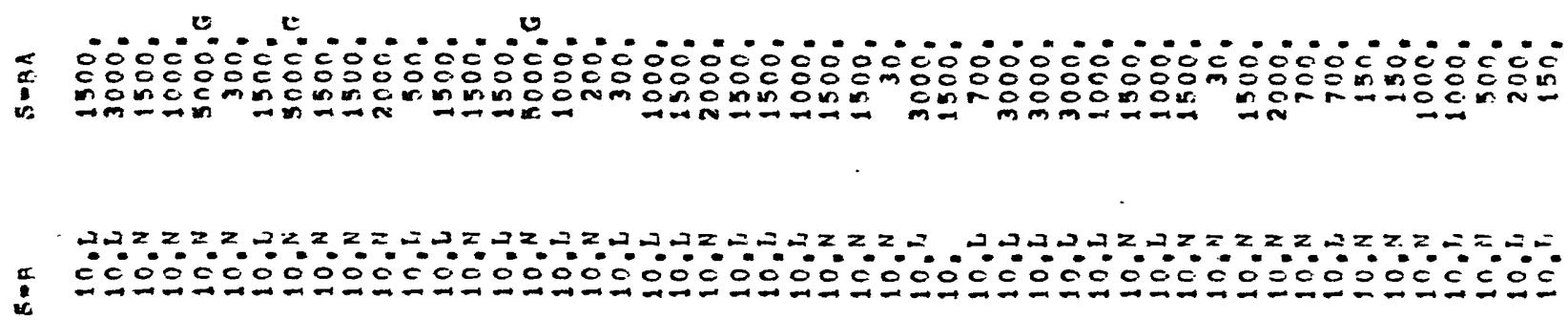

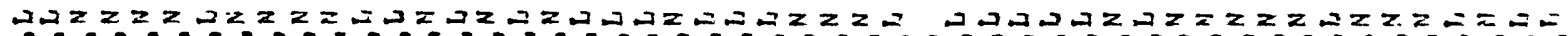
- 500000000000000000000000000000000000000000000 


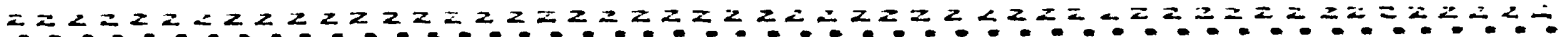

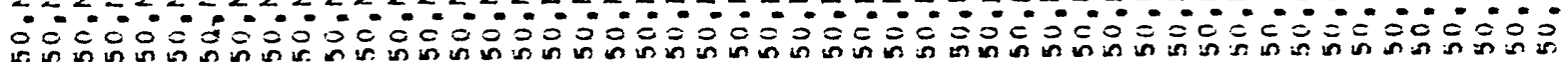

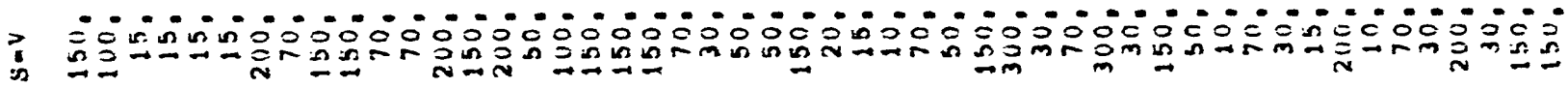

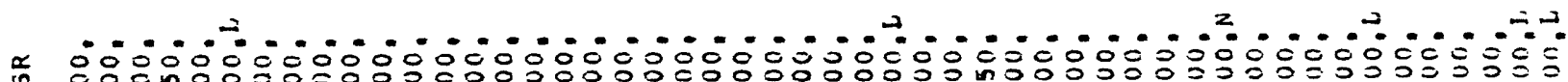

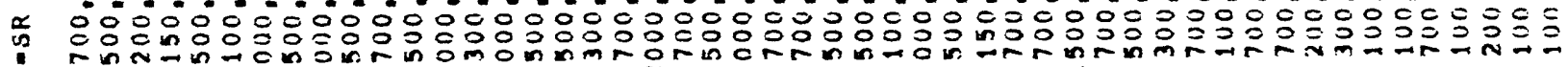
( $\rightarrow$ - $\rightarrow$ -

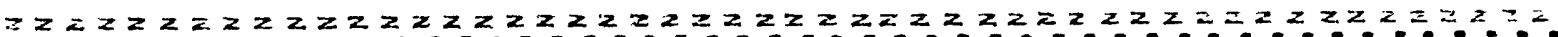

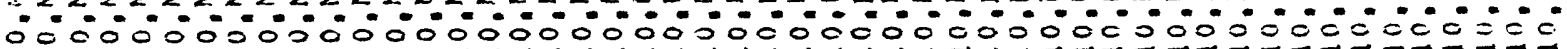

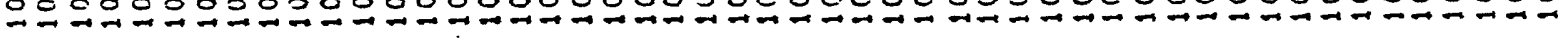

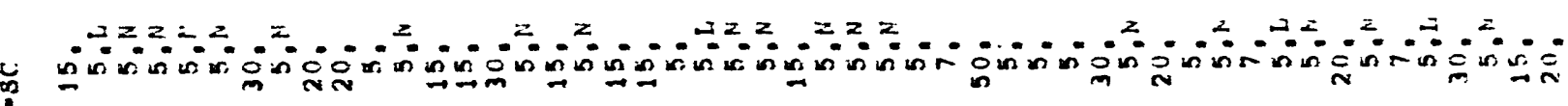
. (2)

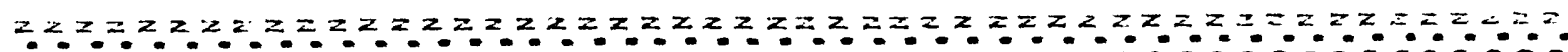

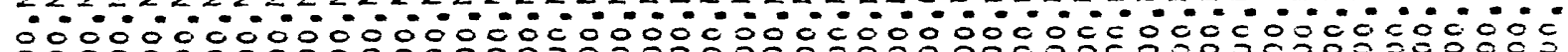

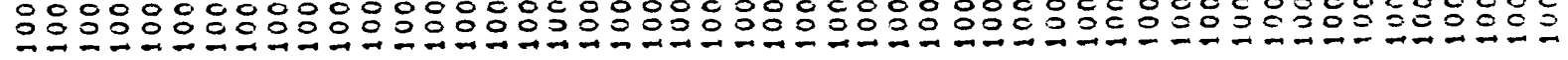

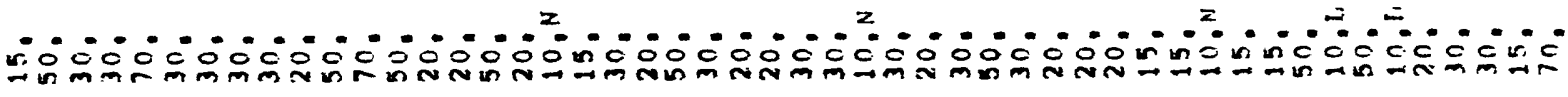

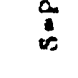
(n)

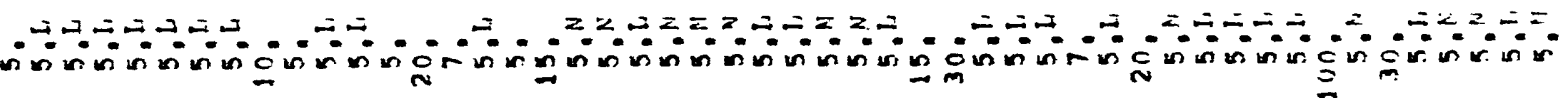




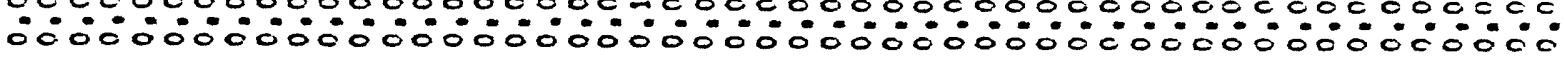

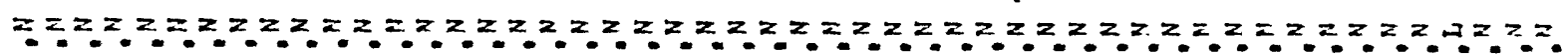

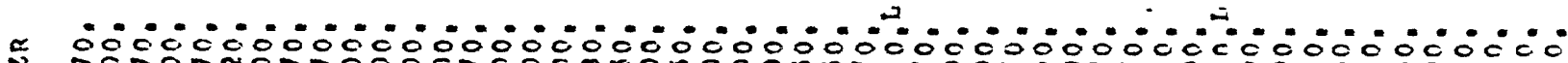

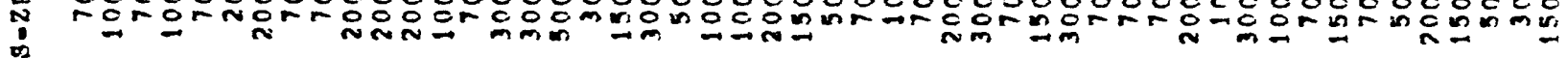




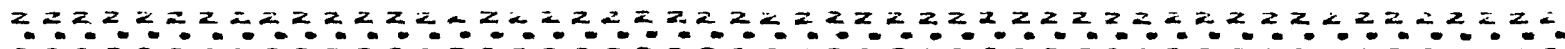

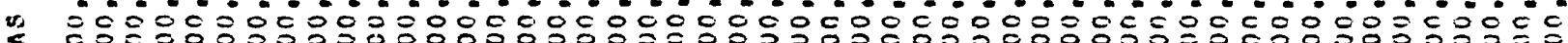

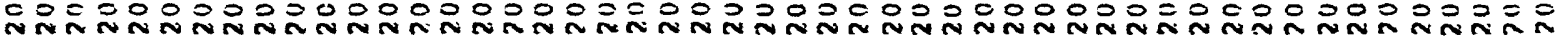

$z z z z z=2, z z z z z z z z z z z$

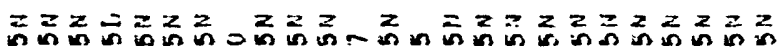

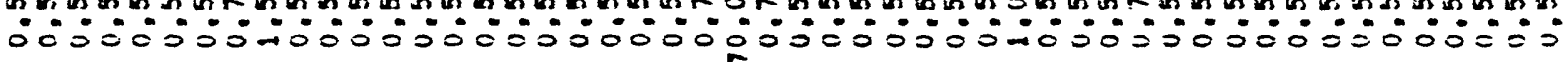

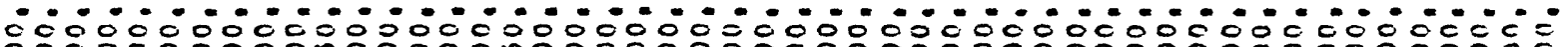

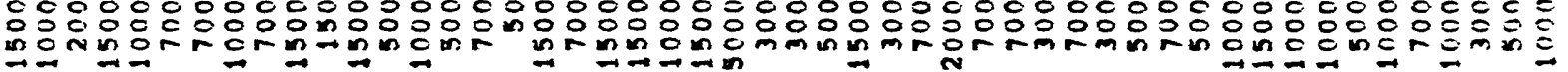

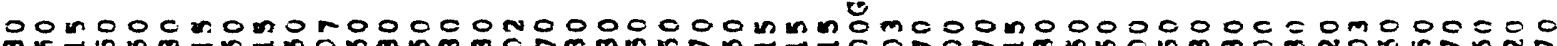

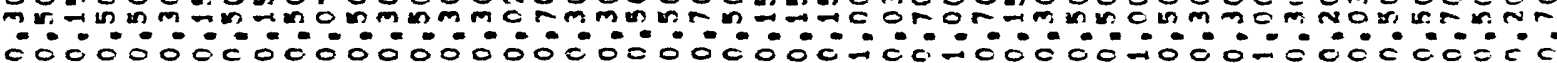

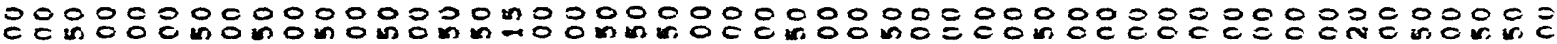

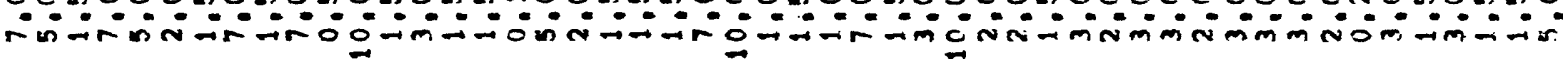

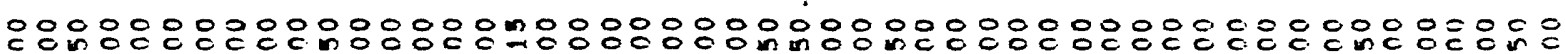

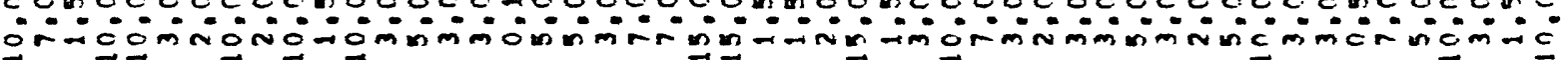

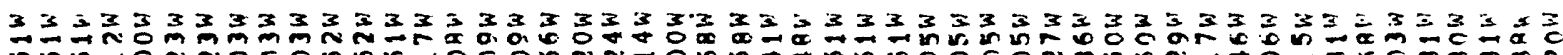

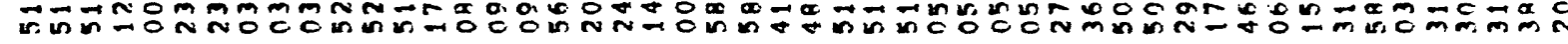

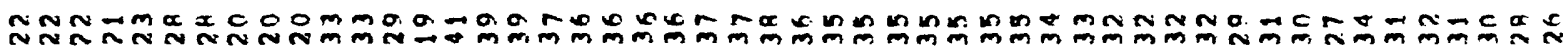
00000000000000000000000000000000000000000000000000

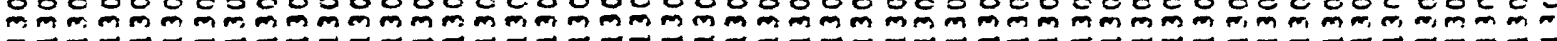

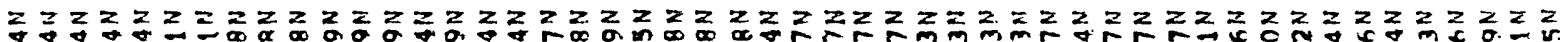

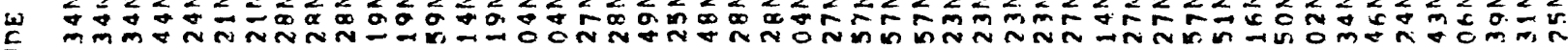

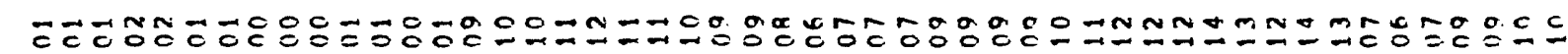

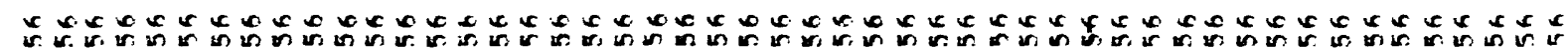

芒

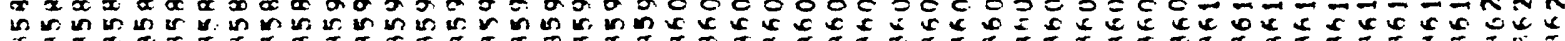

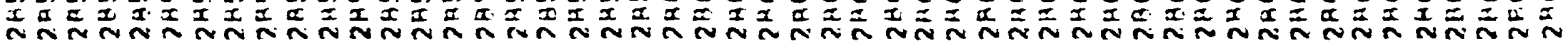

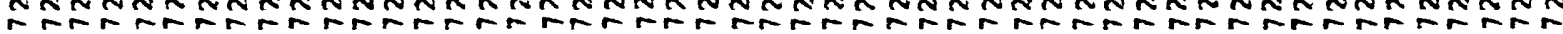




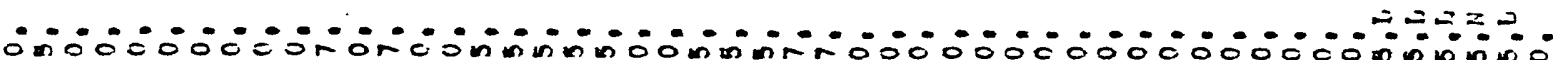

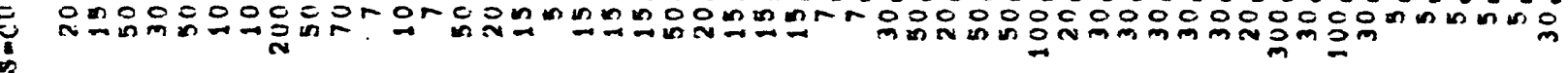

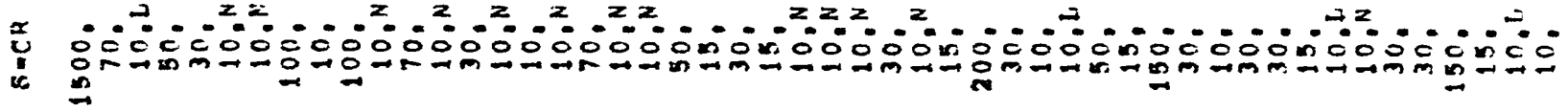

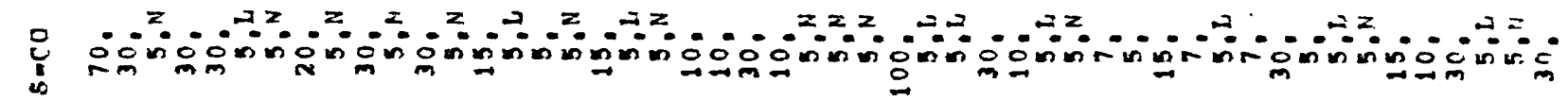

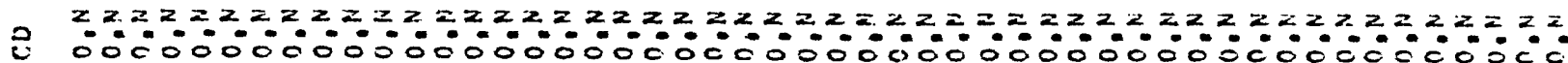

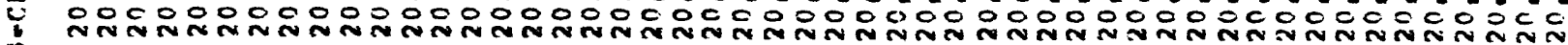

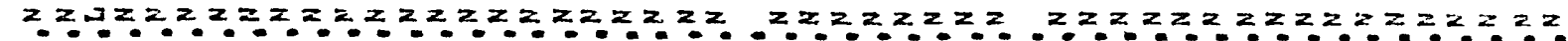
$00000000000000000000000000000000 m 0000000000000000$
\end{abstract}

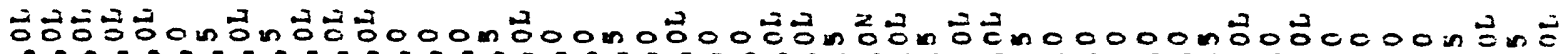

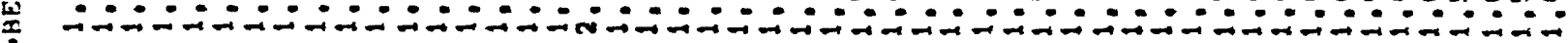

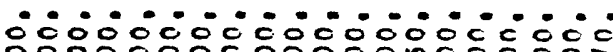

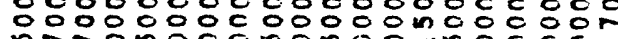
nun

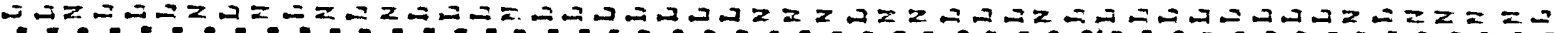

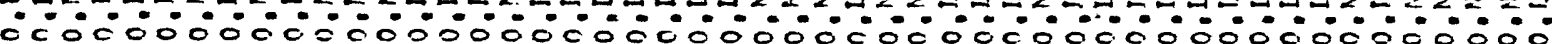




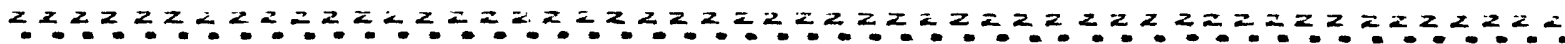

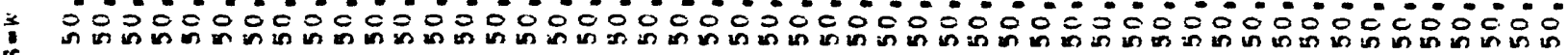

:

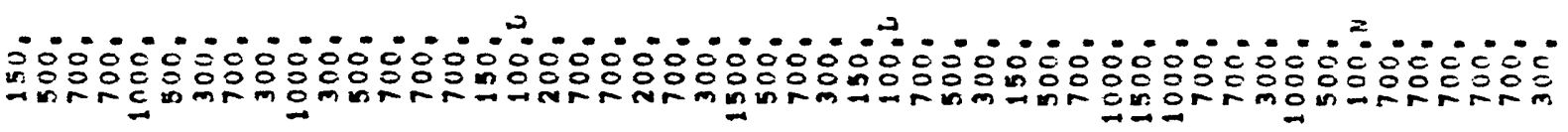

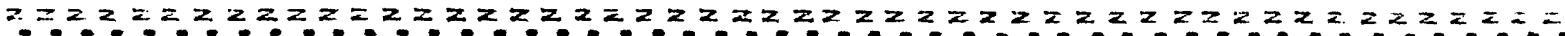
0000 00

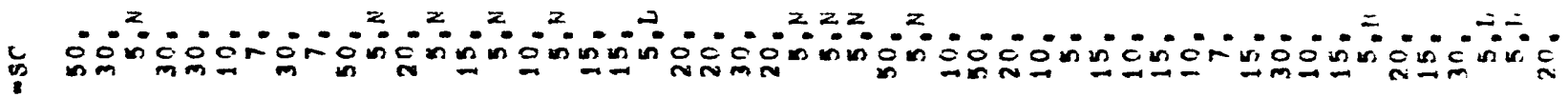

$z z z z z z z z z z z z z z z z=z z z z z z z z z z z z z z z z z z z z z z=2 z z 2 z z z=21$

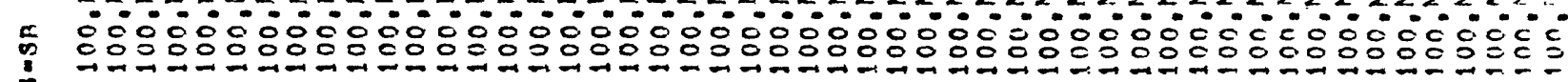

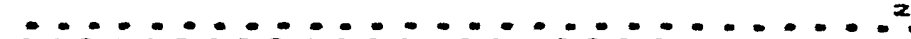

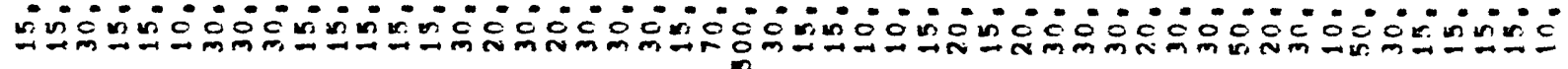 m}

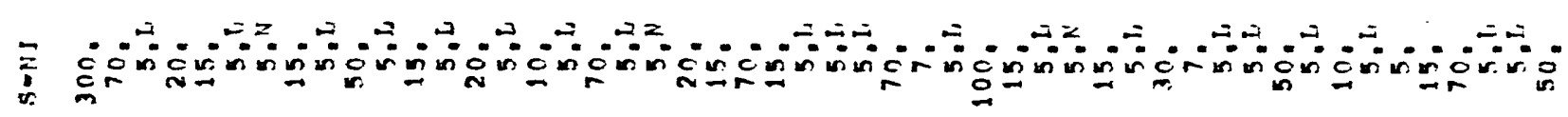
20000000000000000000000000
0 


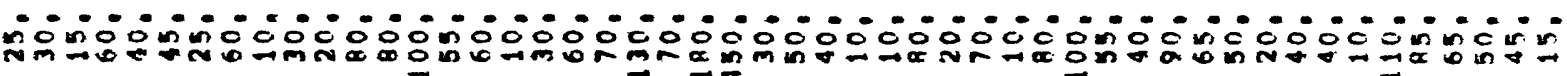
-

:

: nm

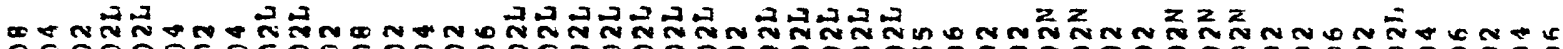
000000000000000000000000000000000000000000000000

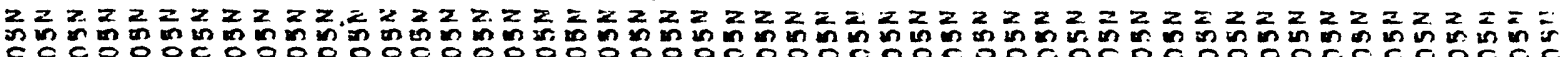

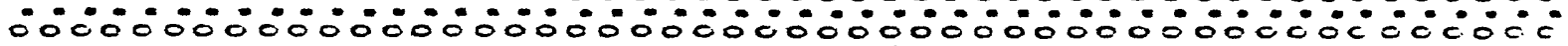

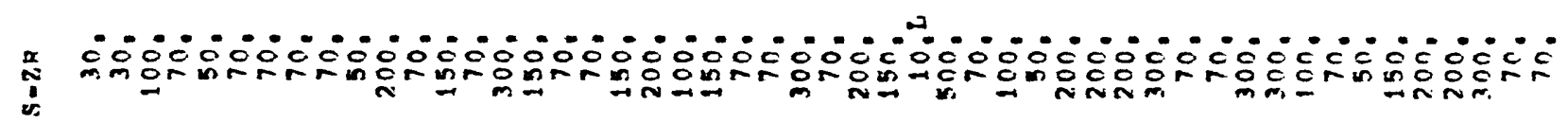

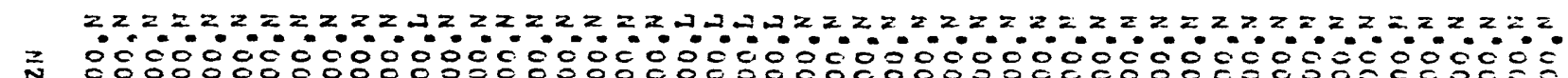

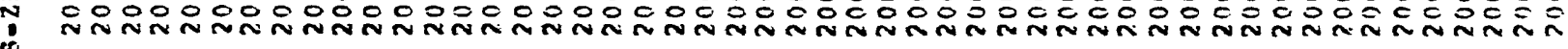

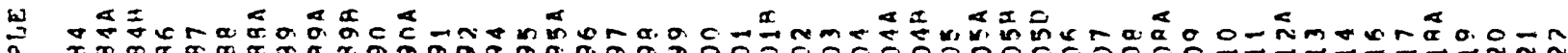
a $\quad$ so

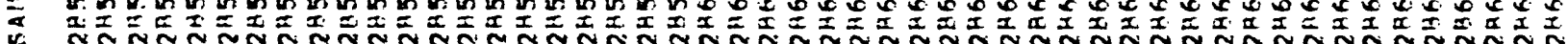

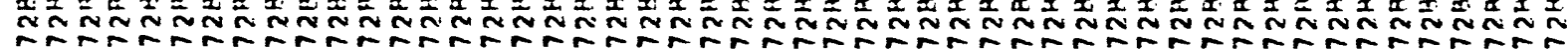




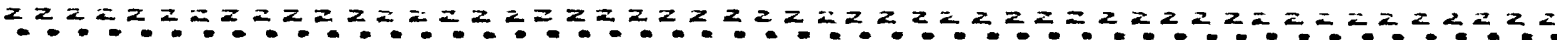

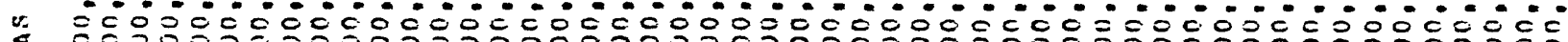

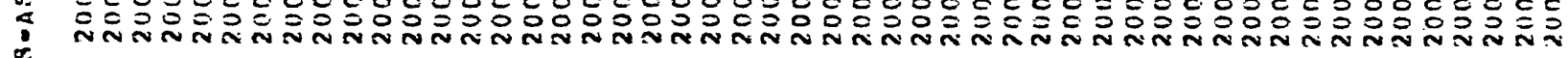

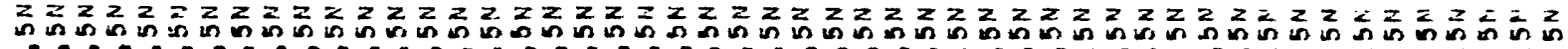

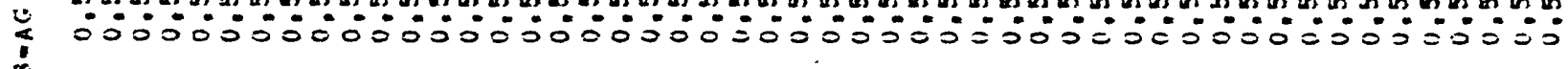

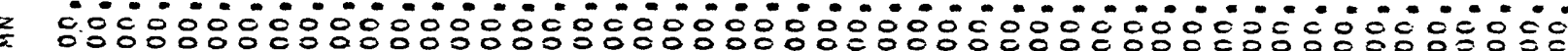

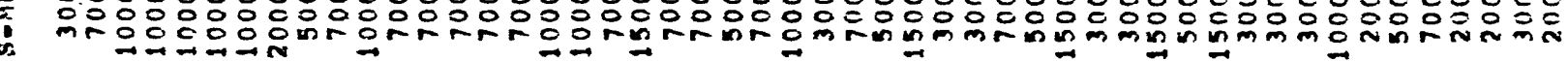

空

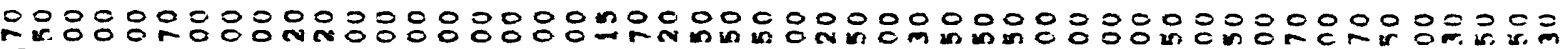

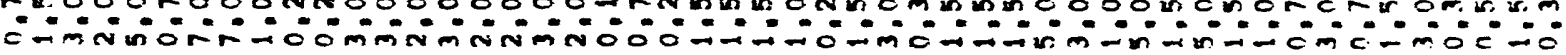

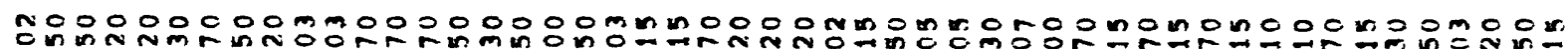

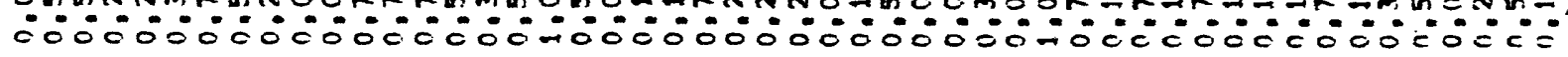

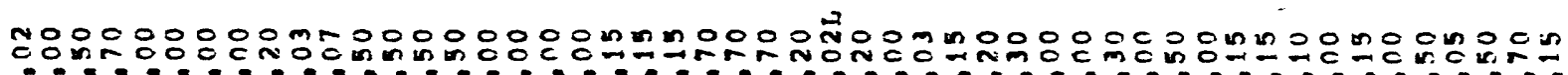

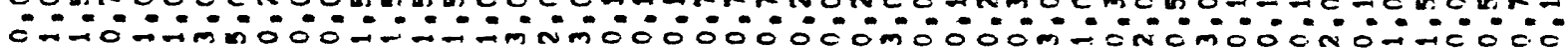

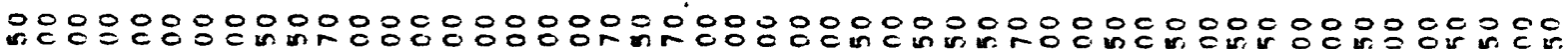

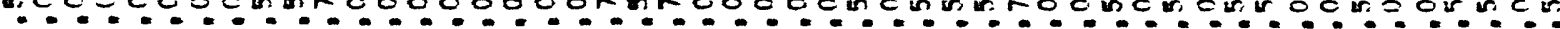

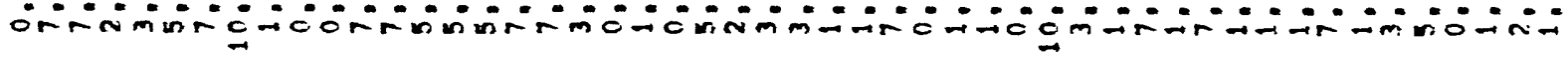

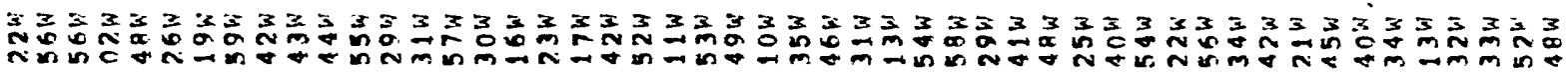

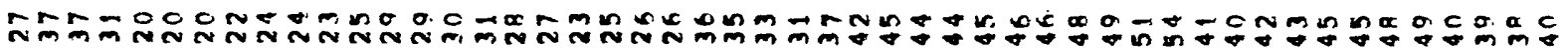

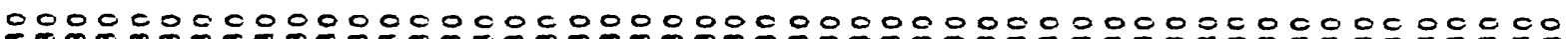

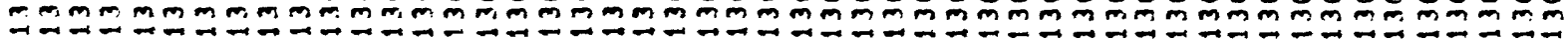

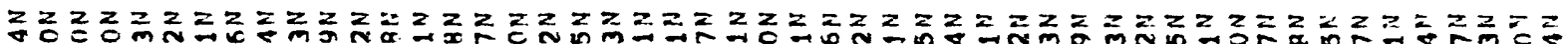
แ

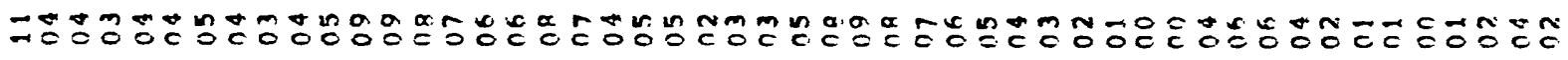

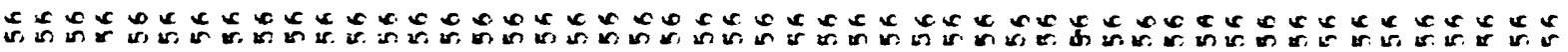

㟧

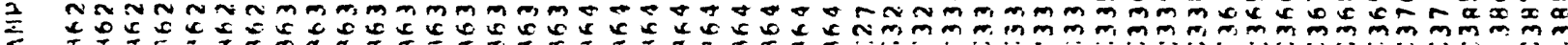

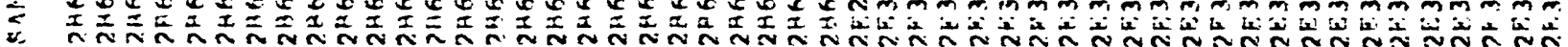

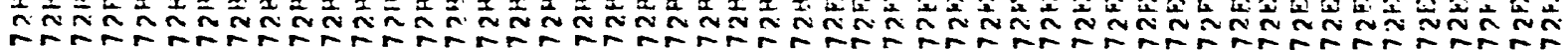




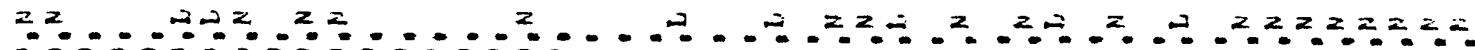

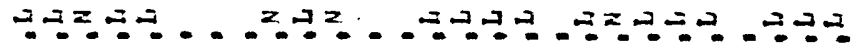

num no

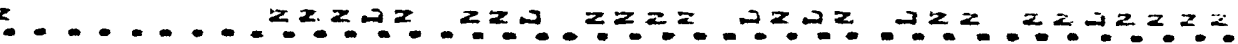

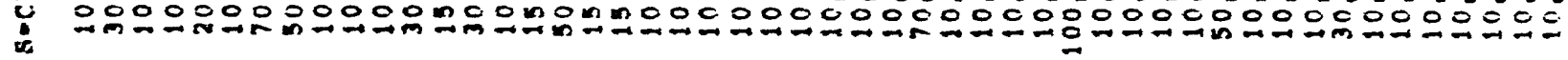

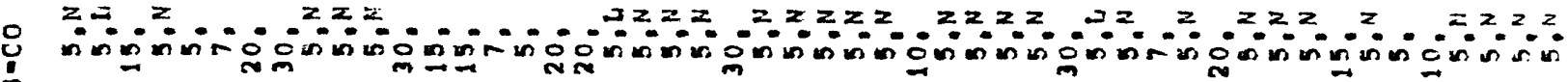

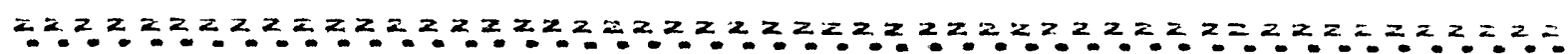

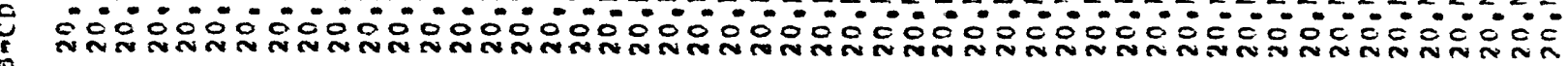

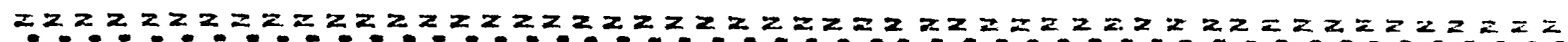

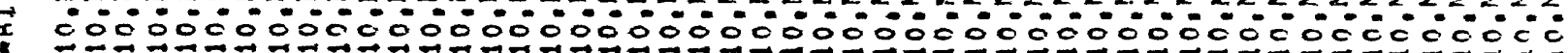
$\infty$

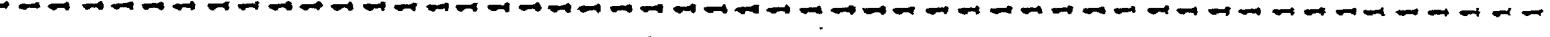

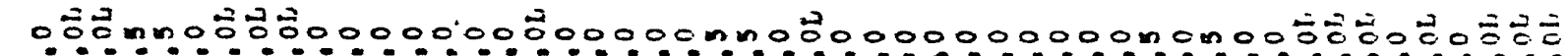
I n

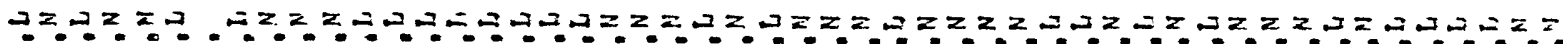
* 000000000000000000000000000000000000000000000000

L m

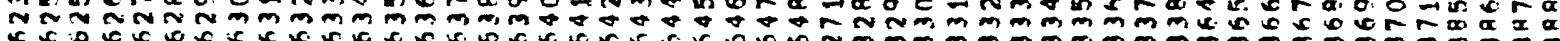

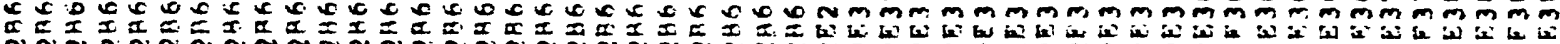

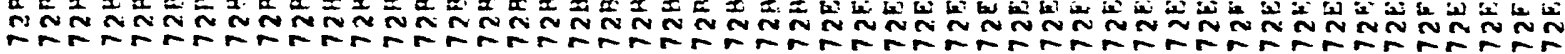




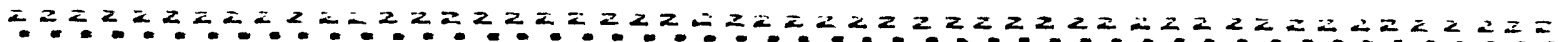

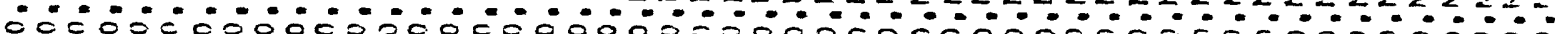

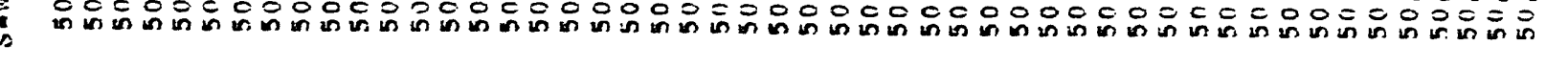

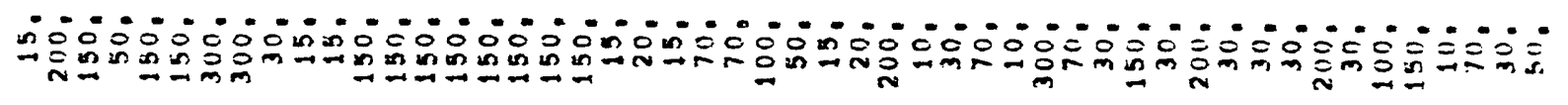

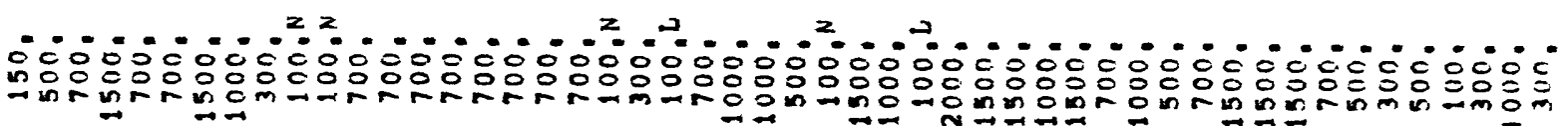

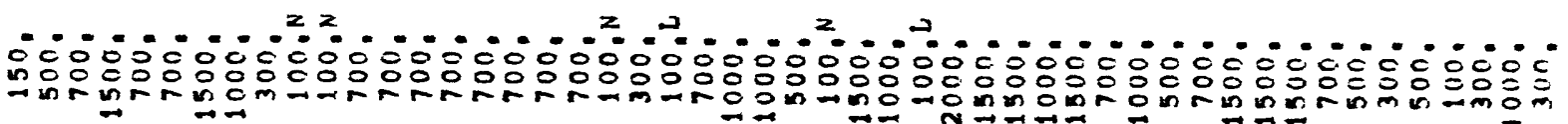

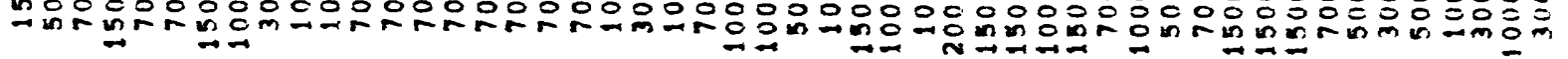

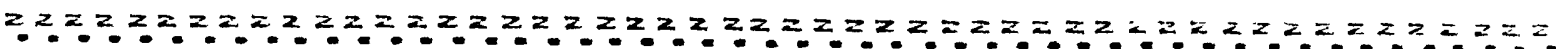
000000000000000000000000000000000000000000000000

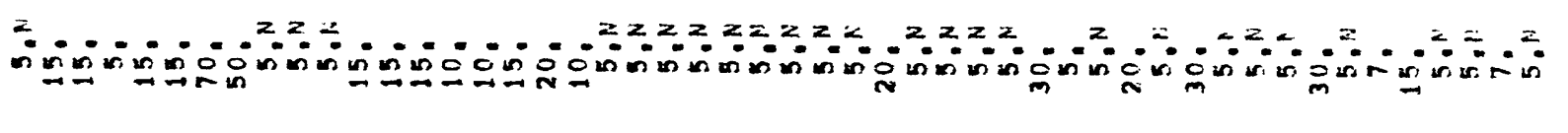

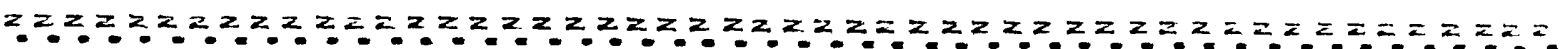

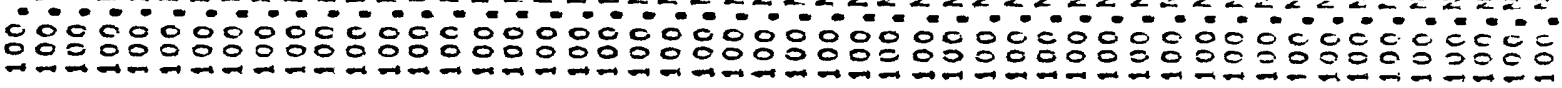

$$
\text { is }
$$

$\frac{\pi}{a}$

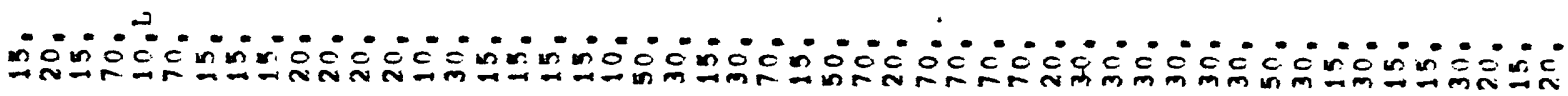

T)

: 


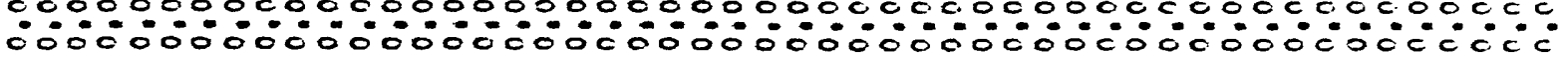

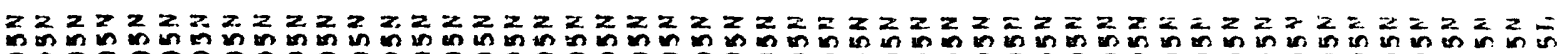

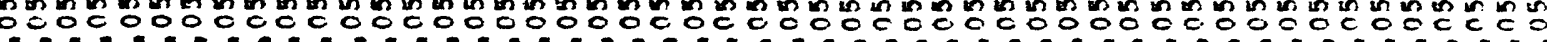

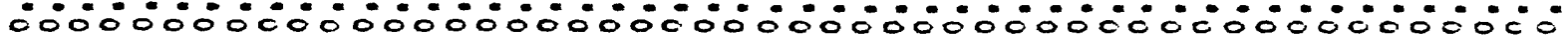

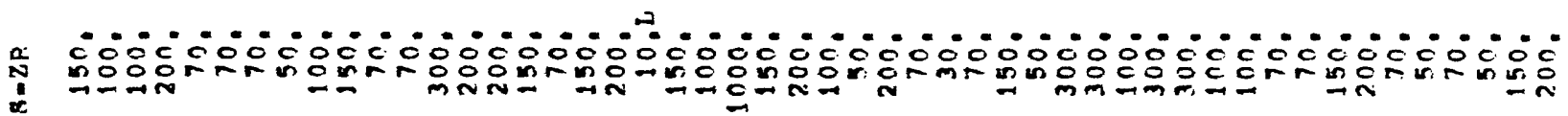

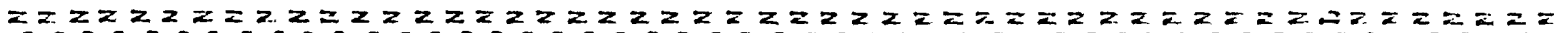
2

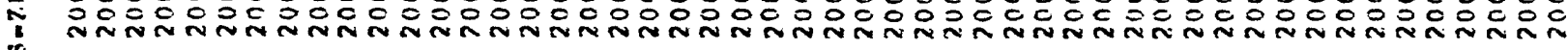




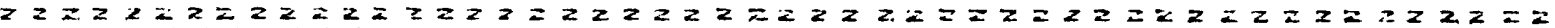

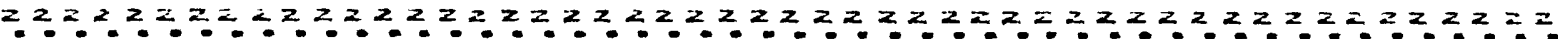

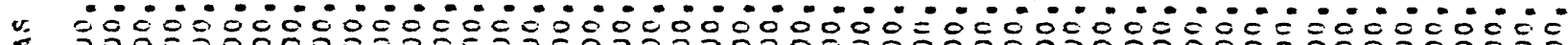

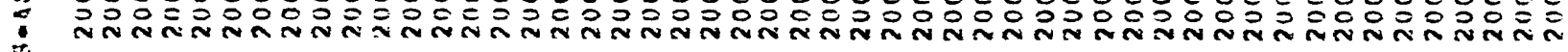

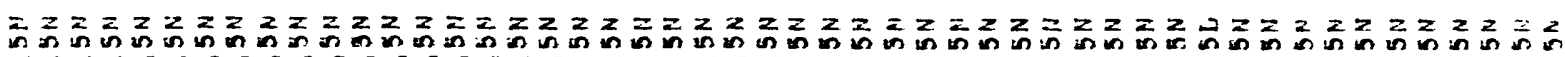
jos



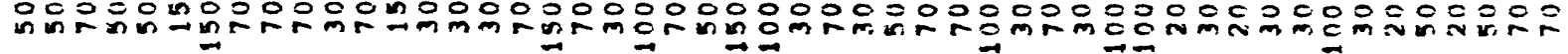

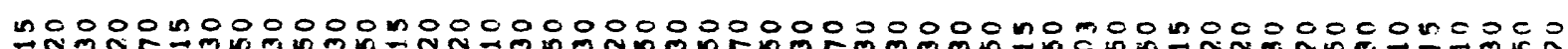

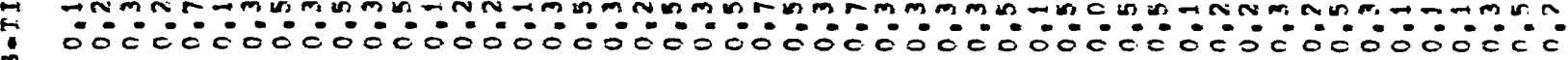

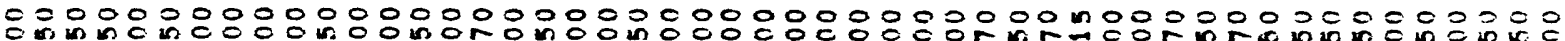

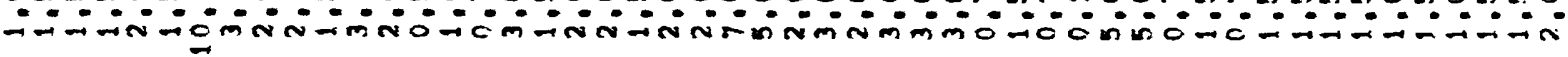

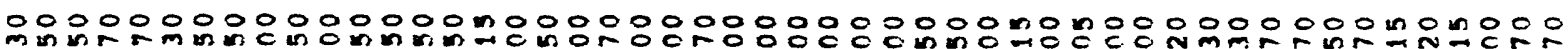

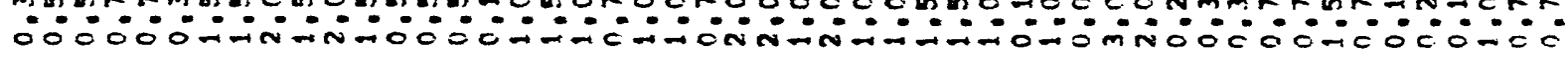

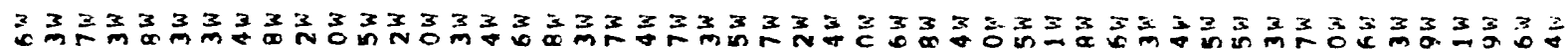

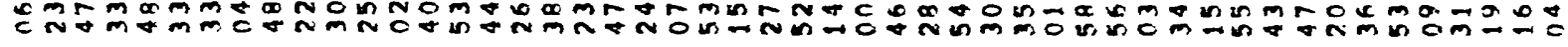

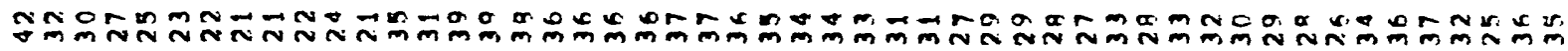

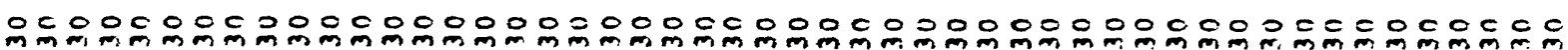

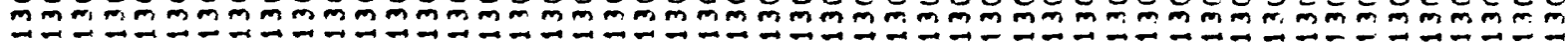

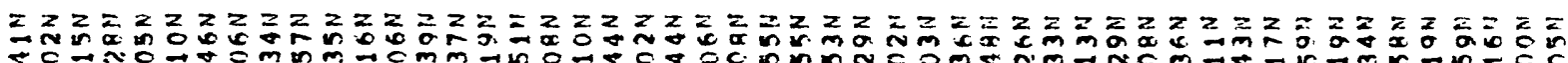

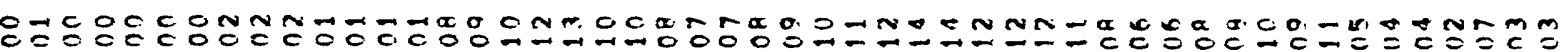

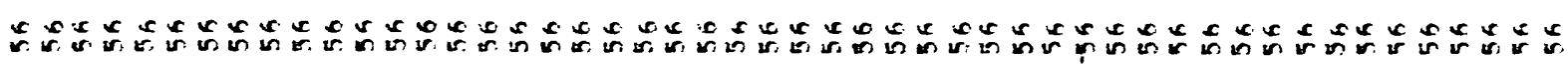




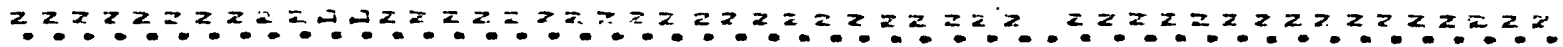

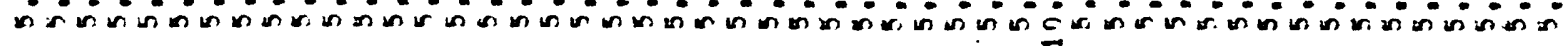

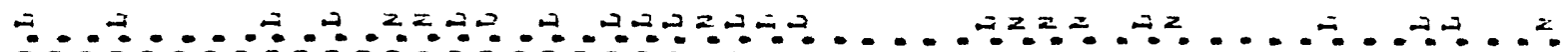

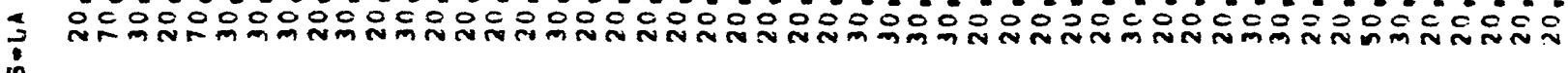

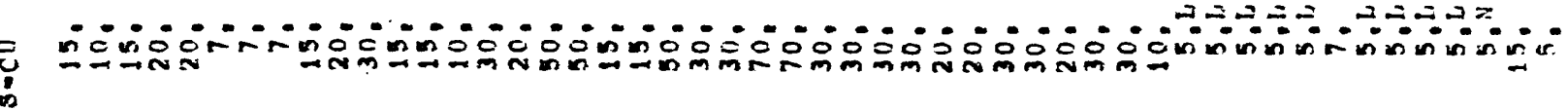

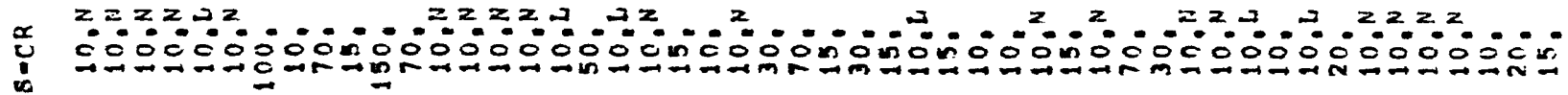

$z z z z z_{0} z \ldots$

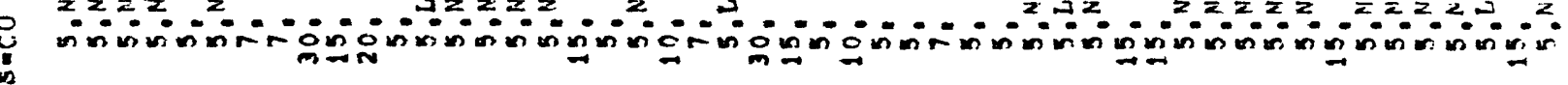

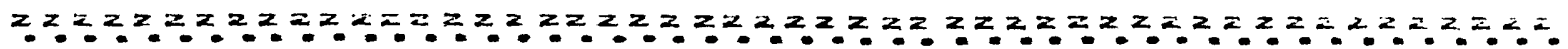

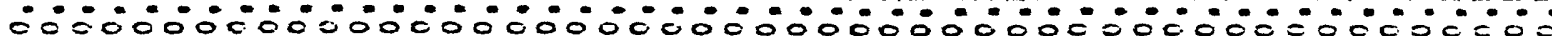

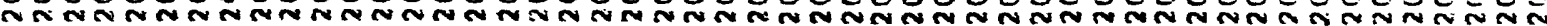

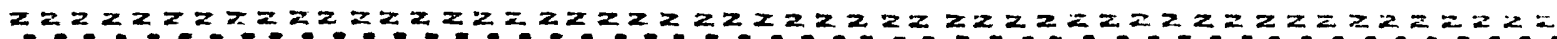

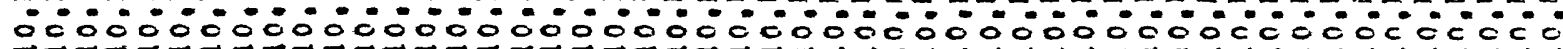

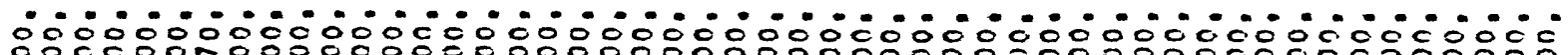

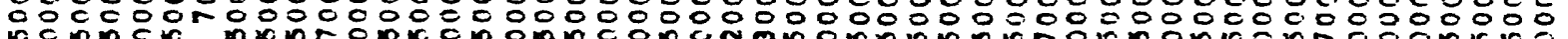

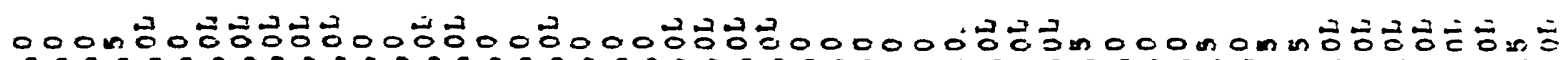

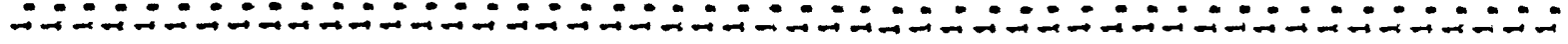

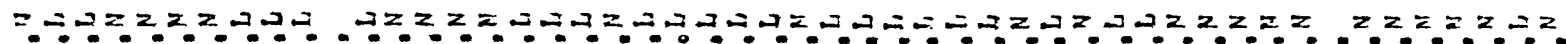
낭

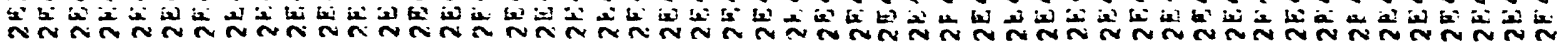
on 


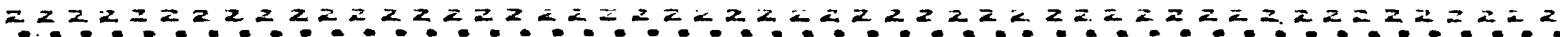

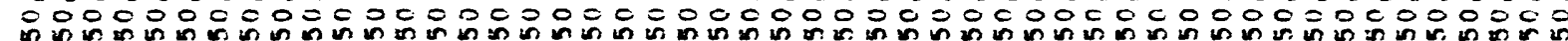

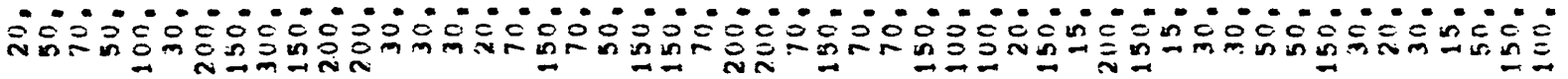

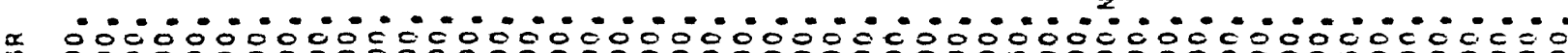

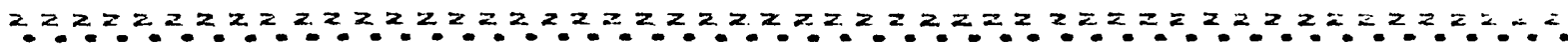
05000000000000000000000000000000000000000000000000

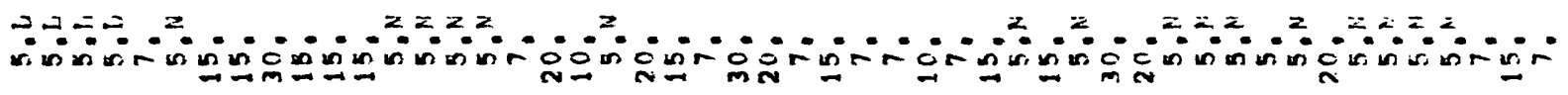

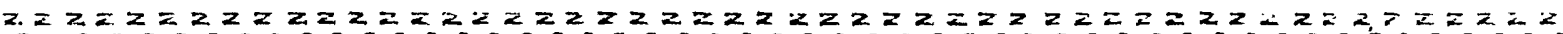

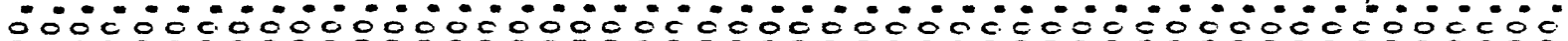

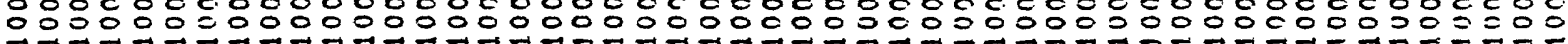
$\therefore \ldots 0^{2} \ldots \ldots+\ldots$

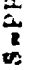

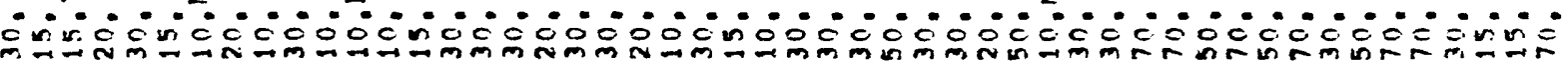

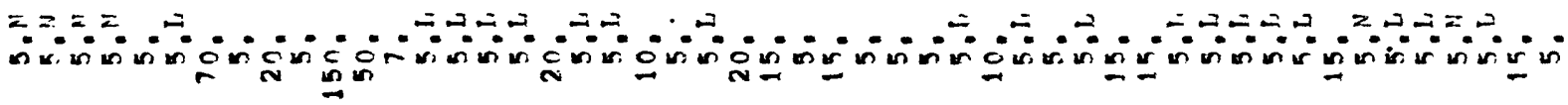

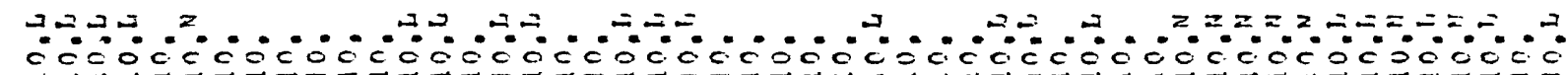

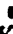
作

c几хо

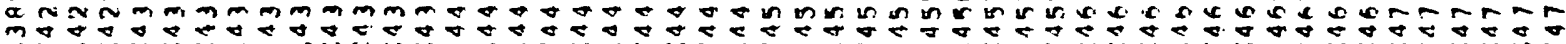

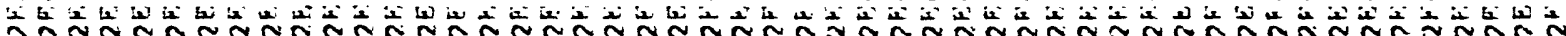

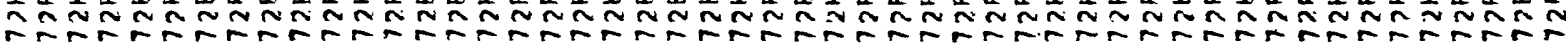




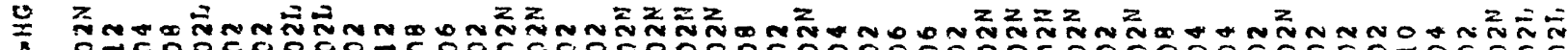
a jo,00000000000000000000000000000000000000000000000

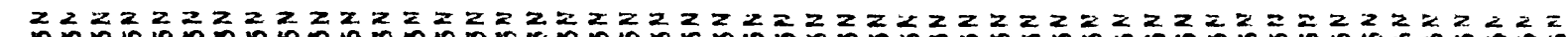

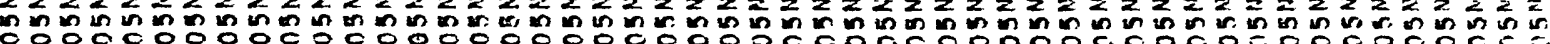
000000000000000000000000000000000000000000000

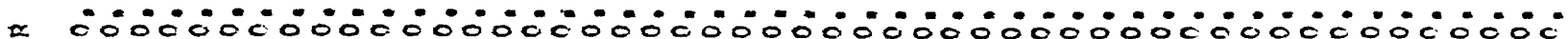

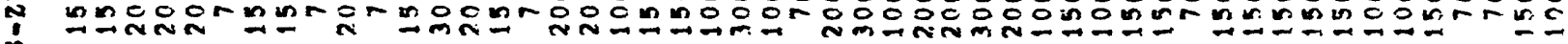

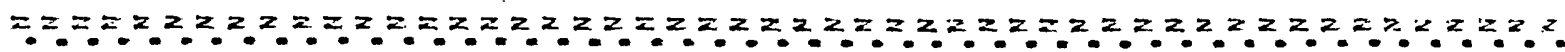

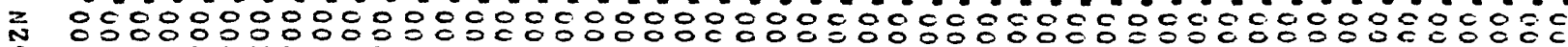

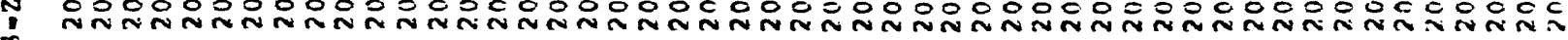

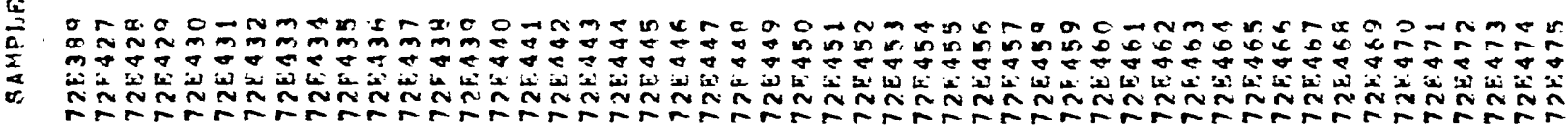




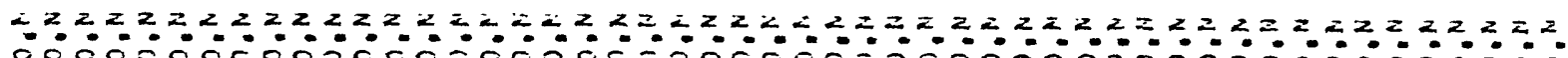

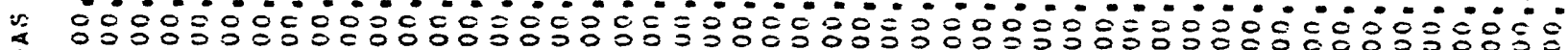

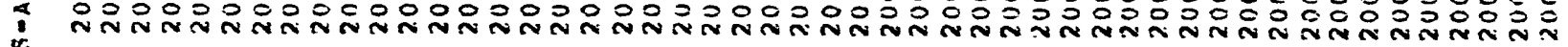

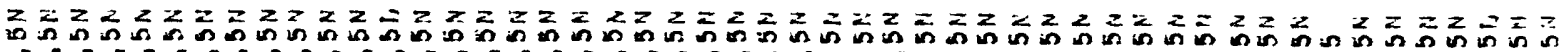

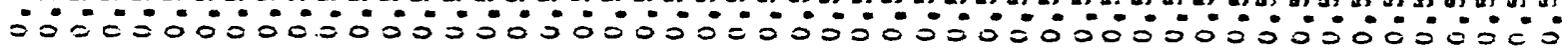

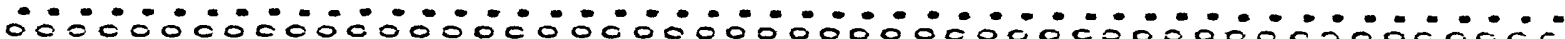

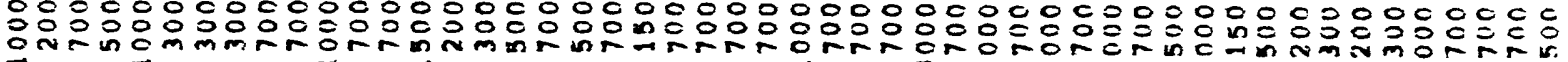

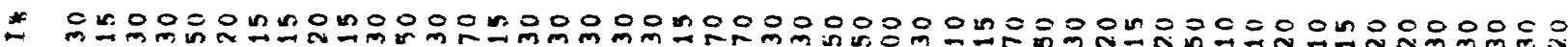
F

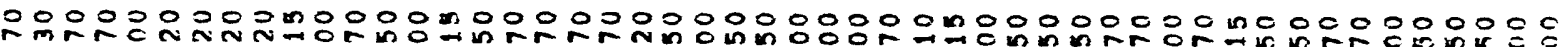

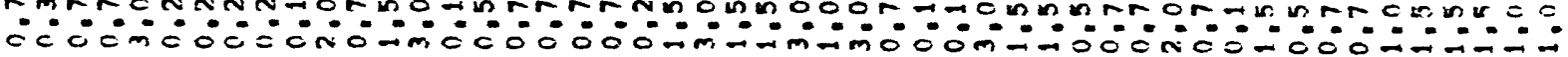

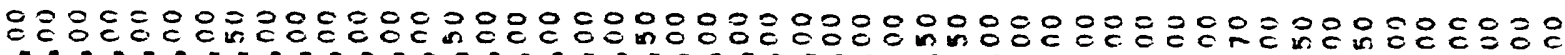

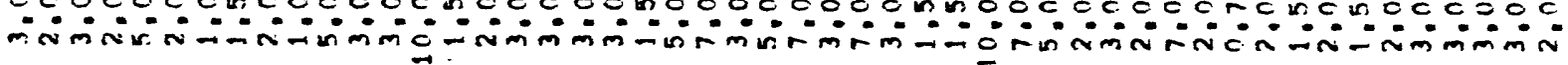

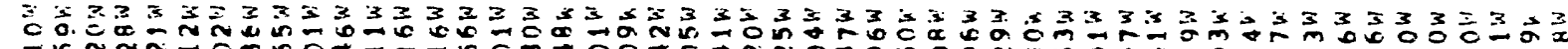

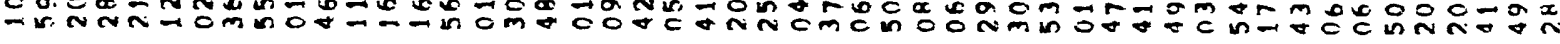

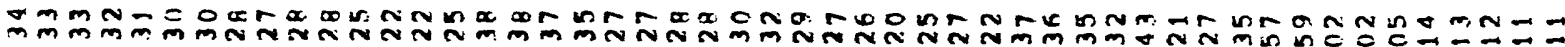

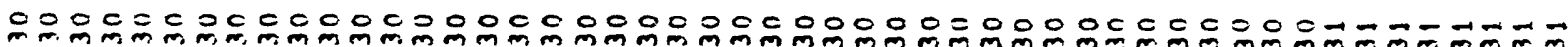

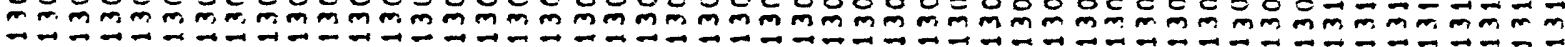

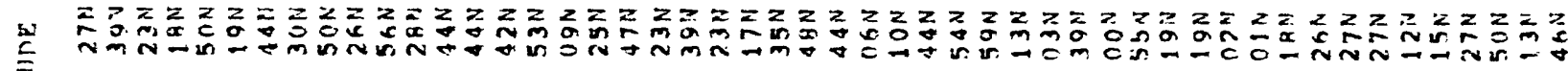

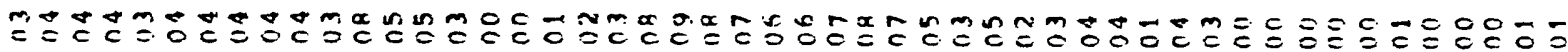

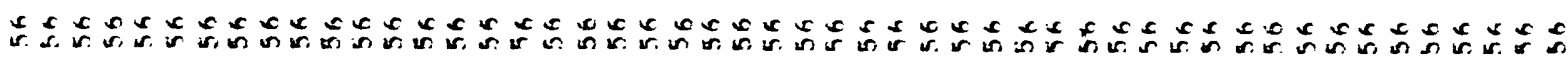




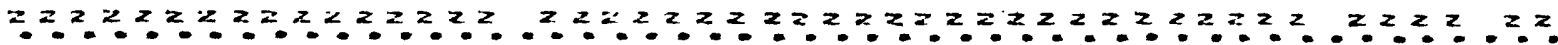
sins

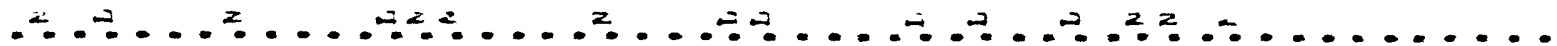

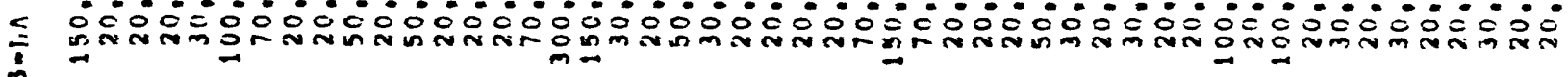

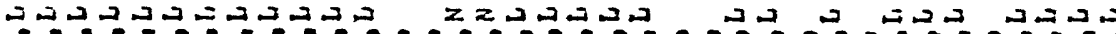

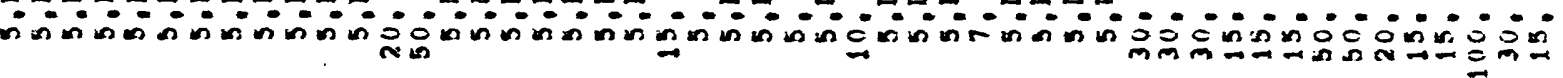

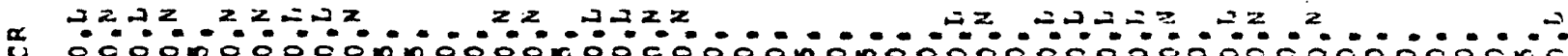

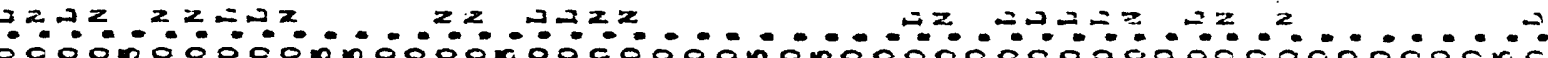

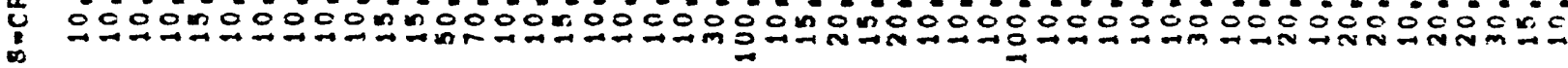

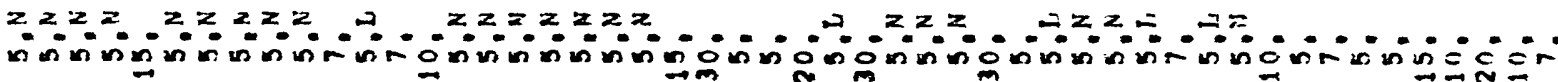

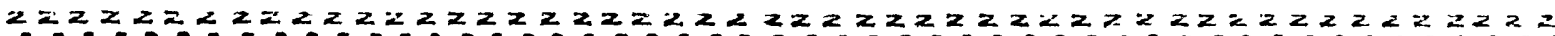

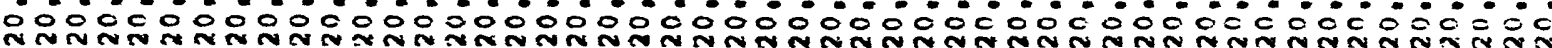

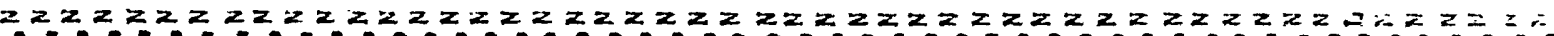
- 0000000000000000000000000000000000000000000

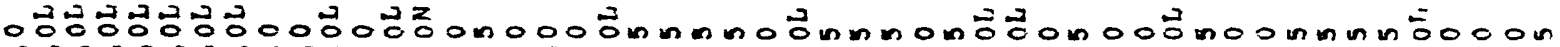
:

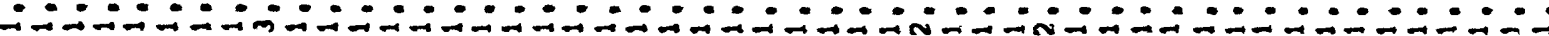

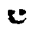

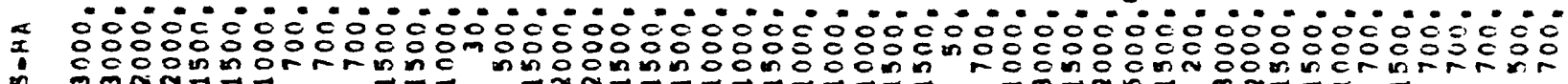
$\min \pi \rightarrow$

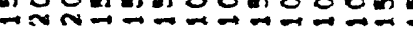

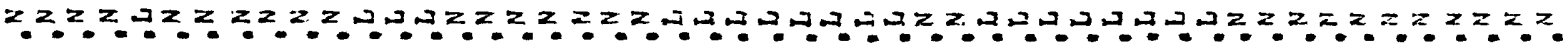
- 00000000000000000000000000000000000000000000000000

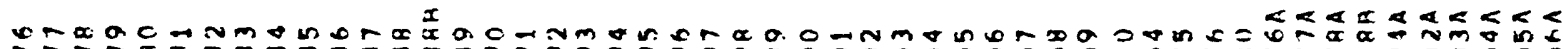

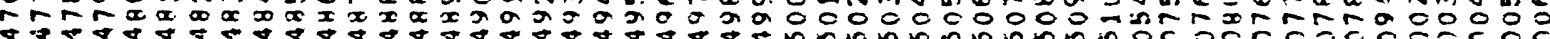

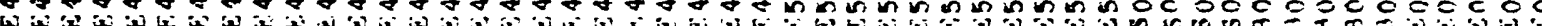

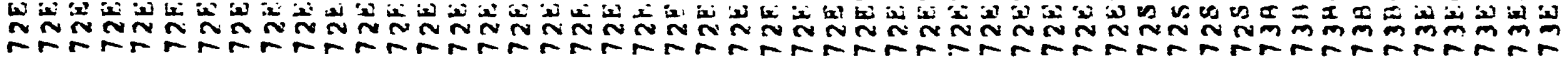




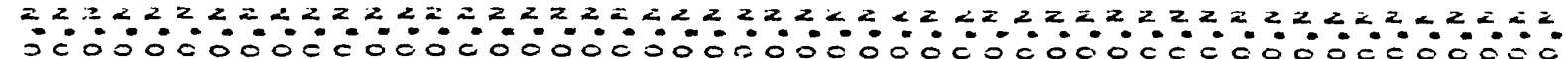

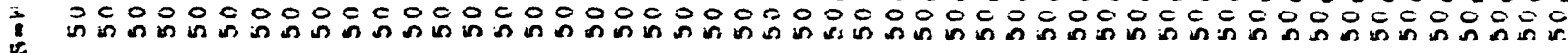

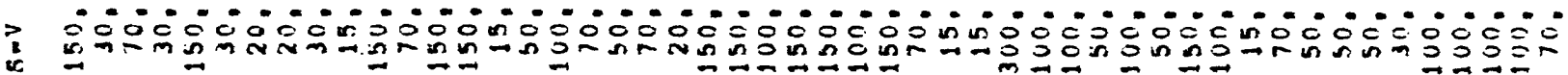
$\rightarrow-1$

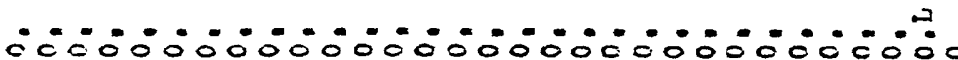

: cor $r$

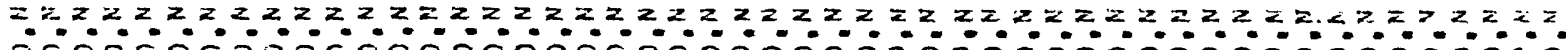
$\sqrt{8}$

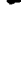

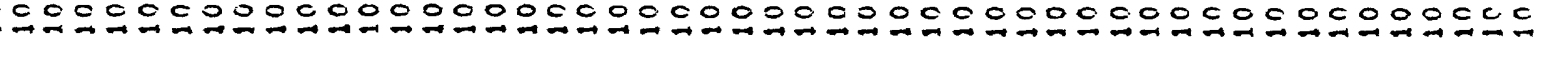

:

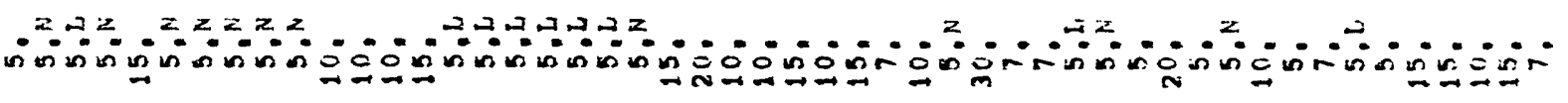

\section{$z z z z z z z z z z z z z z z z z z z z z z z z=z z z z z z z z z z z z z z z z z 2 z z=2 z z z$

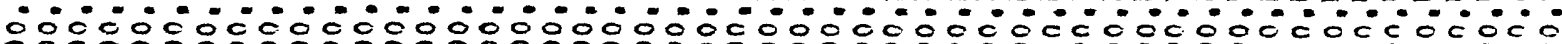

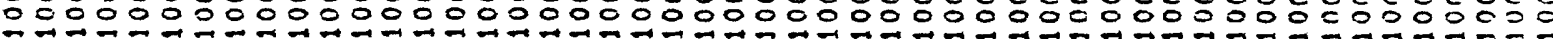

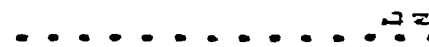

\section{$\geq=$} $=$

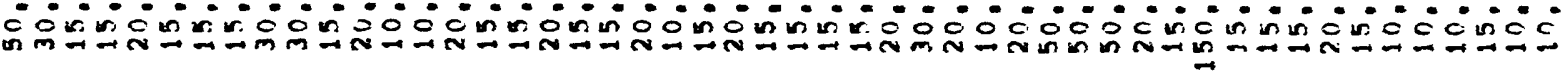

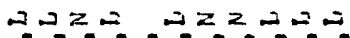
$2 z=x \geq z=2$ rivisuminum

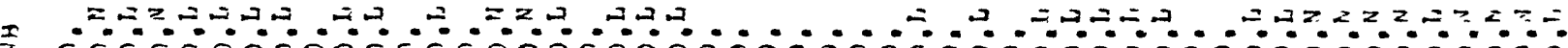
z $\quad$ I

nhx

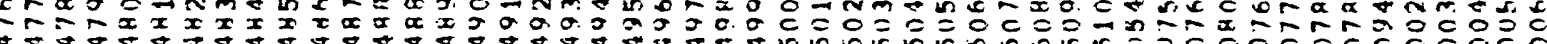

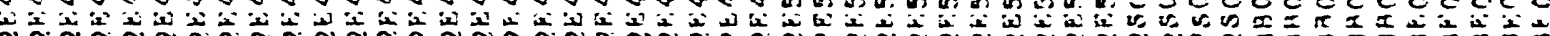

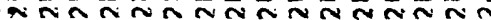




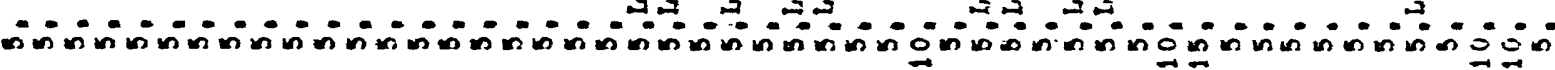

:

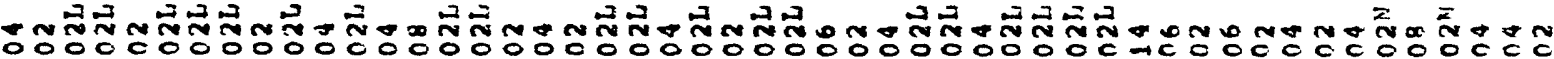
000000000000000000000000000000000000000000000

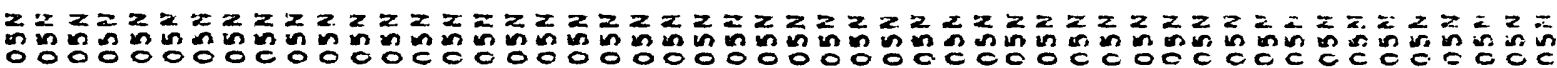
c000000000000000000000000000000000000000000000

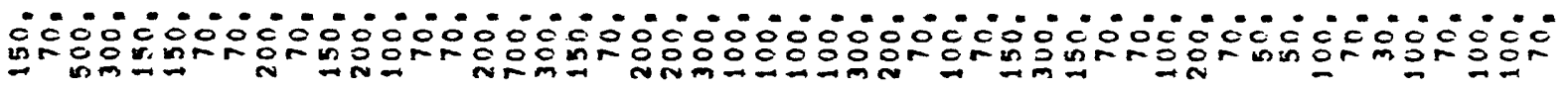

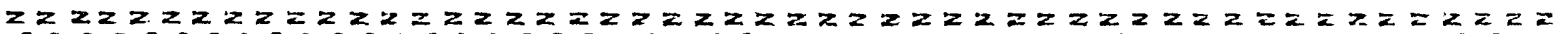

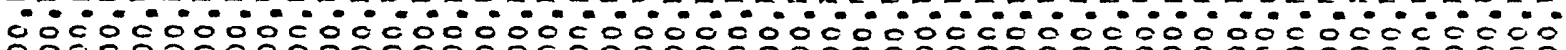

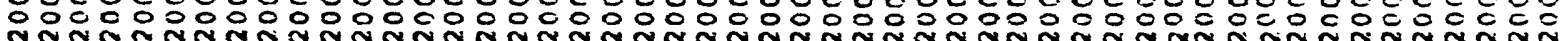


$z z z \geq z z \geq z z z z z z z z<z \geq z z=2=2 z=2 z$

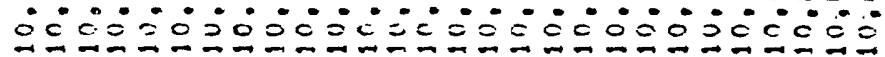

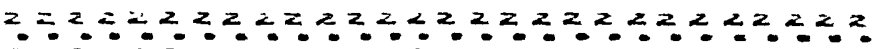

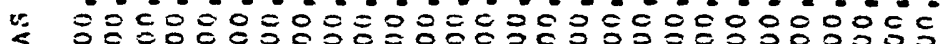

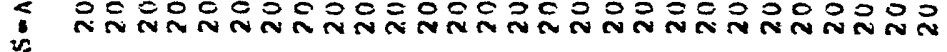

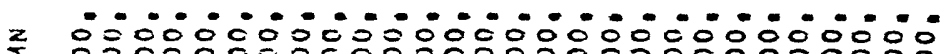
* 궁의

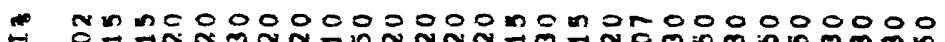
$\because 0000000000000000000000000$

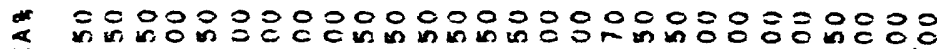
i $0-11-10 \div$ m

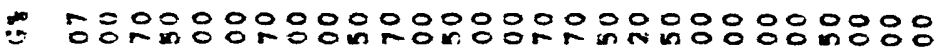

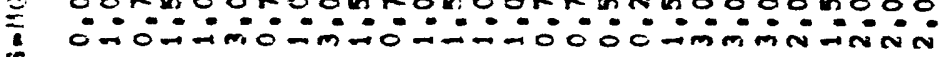
O-

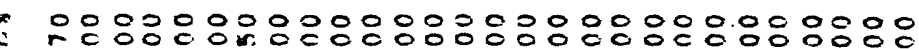

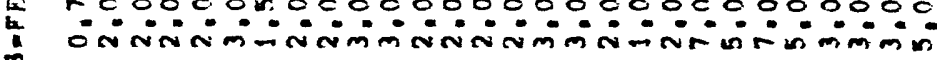

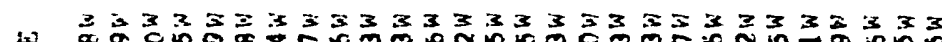
c N 은

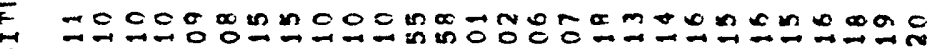
$\vec{m} \vec{m} \vec{m} \vec{m} \vec{m} \vec{m} \vec{m} \vec{C} C \vec{m} \vec{m} \vec{m} 000000000 c 0$

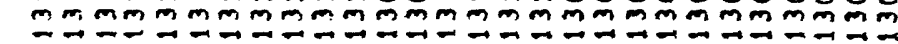

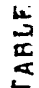

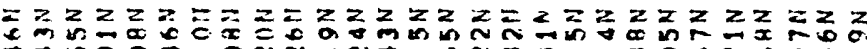

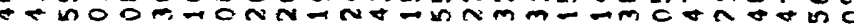

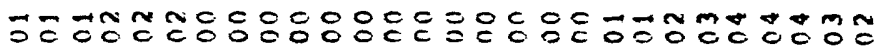

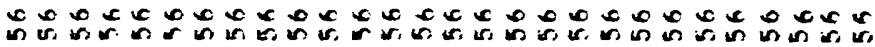




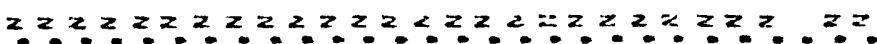

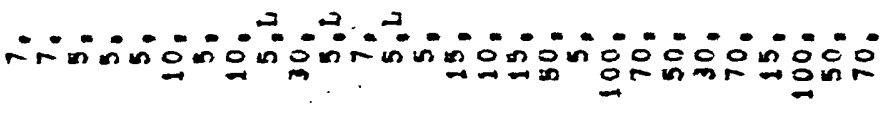

$\geq \geq 2$

눙

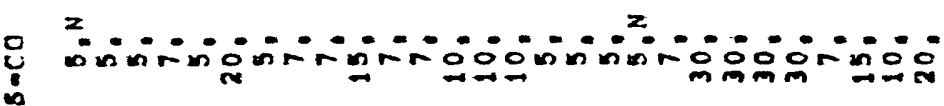

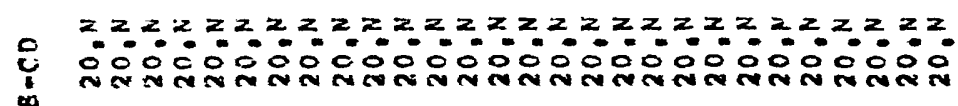

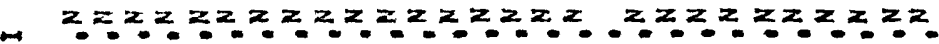
$\overrightarrow{0} 0000000000000000000000000$

$\overrightarrow{2}$

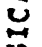

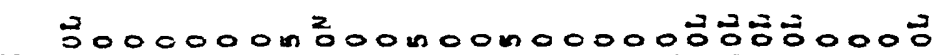

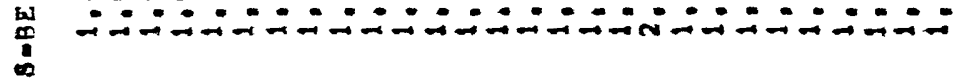
$\infty^{\infty}$

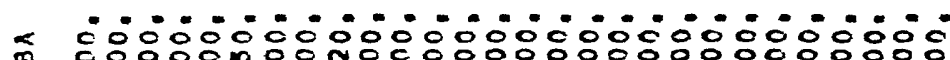

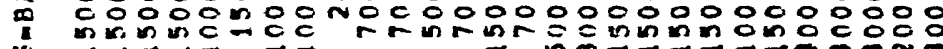




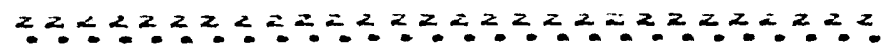

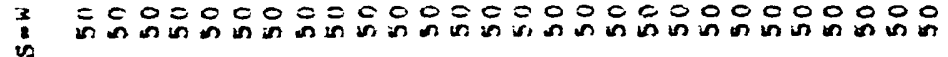

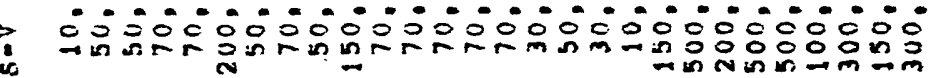

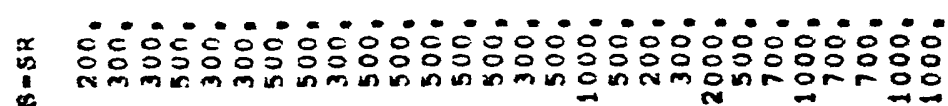

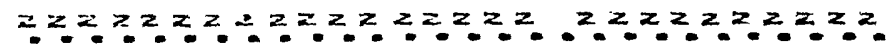

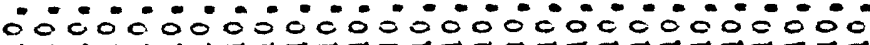

is

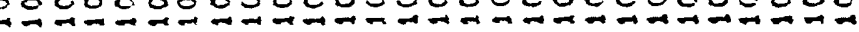

$\leq$

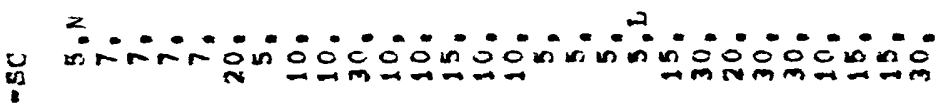

$\stackrel{10}{21}$

$z z z z z z z z z z z z z z z z z z z z z z z z z z z:$

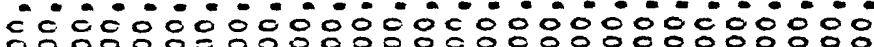

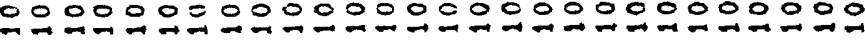




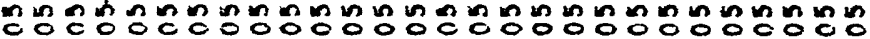

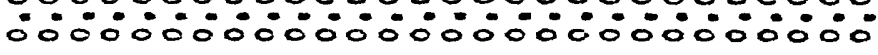


$z z z z z z z z z z z z z z z z z z z z z z z z$ zzzzzzzzzzzzzzzzzzzzzzzzz $\stackrel{\gtrless}{i}$

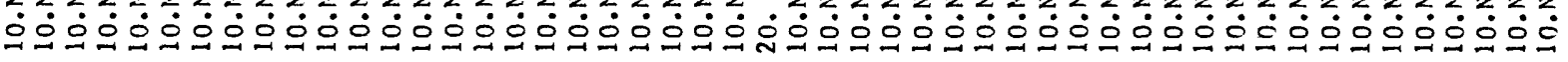

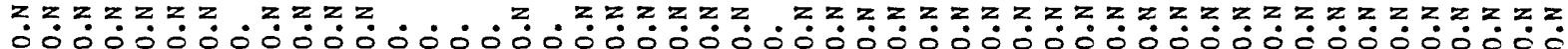

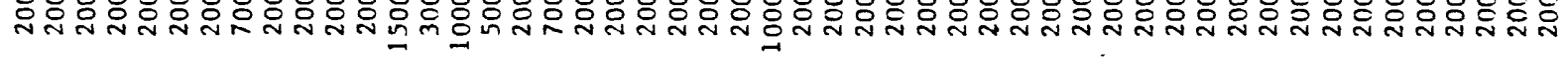
少

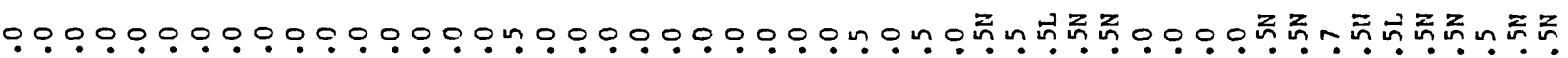

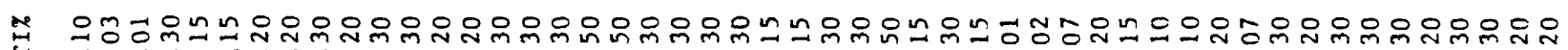
着

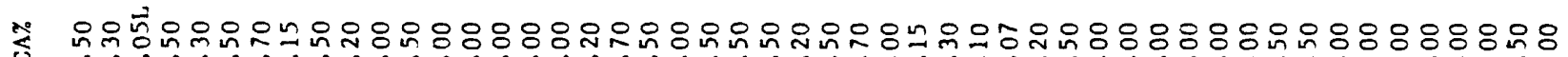

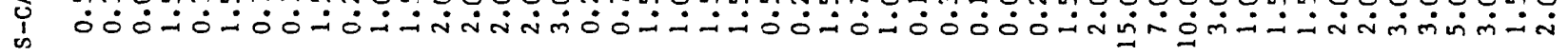

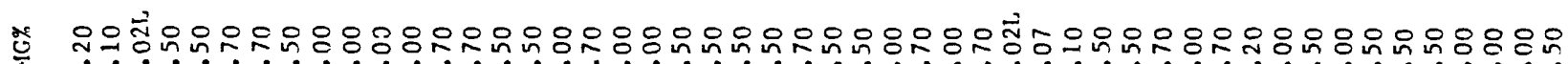
ஸे

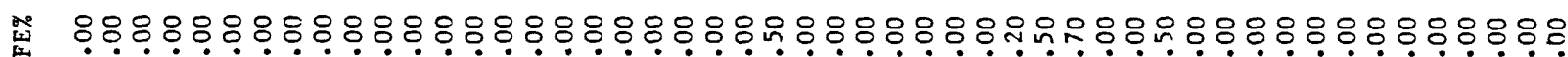

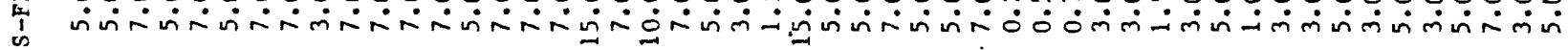

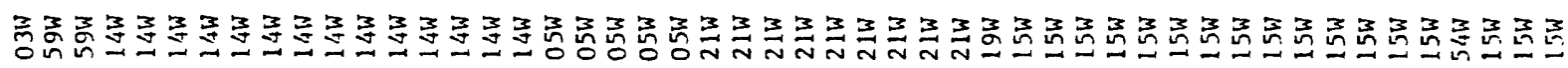

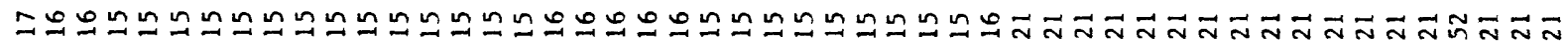

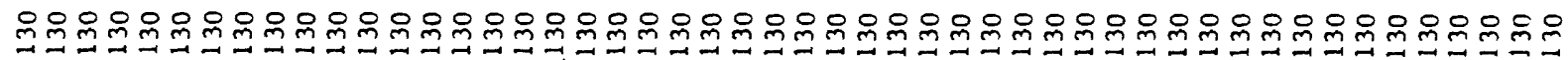

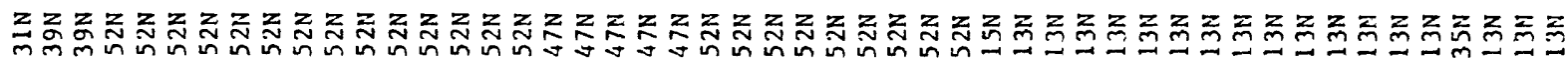

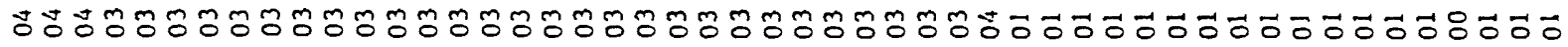

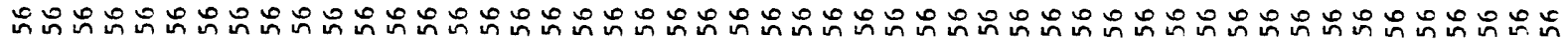

$\sum_{\text {ह }}^{5}$

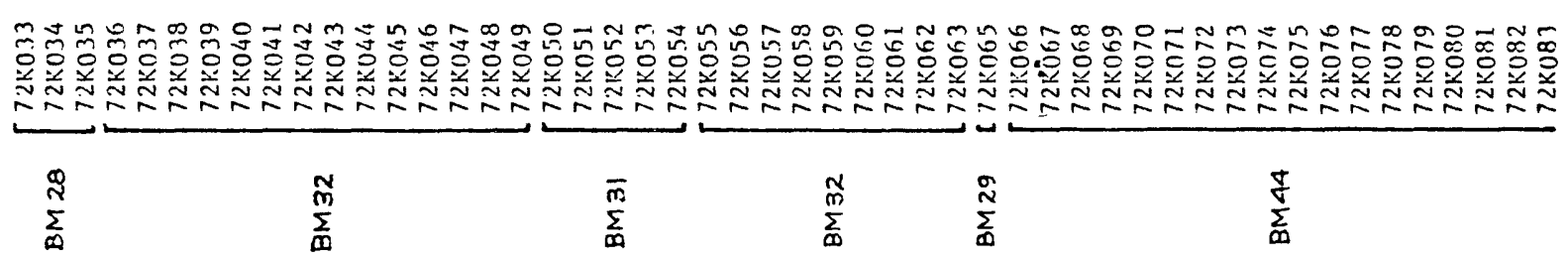




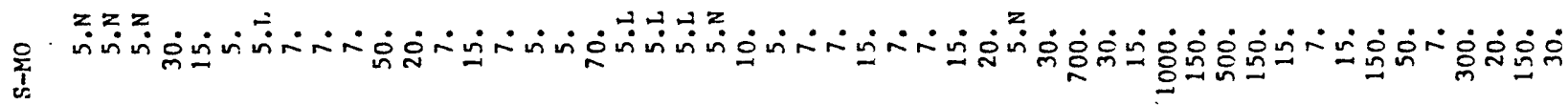

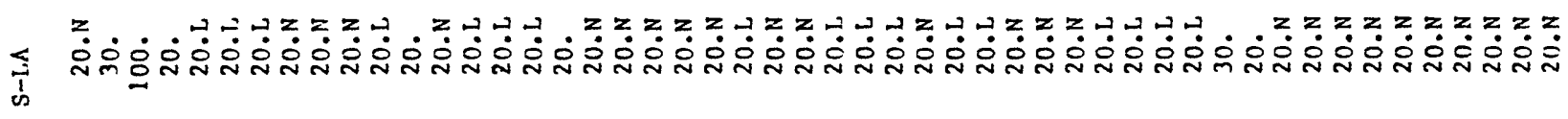
ن

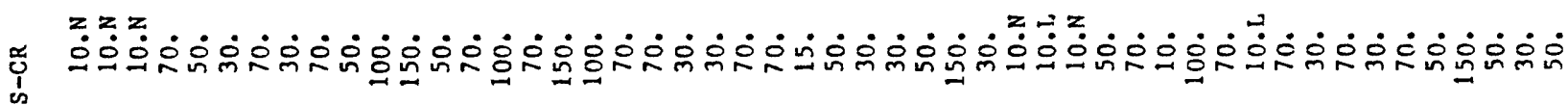

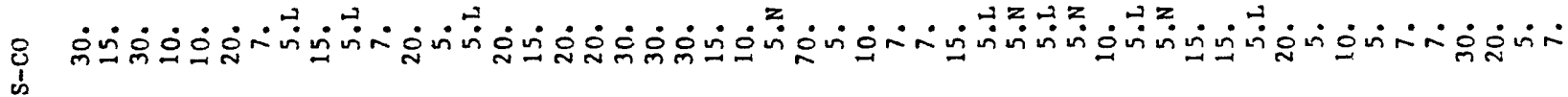

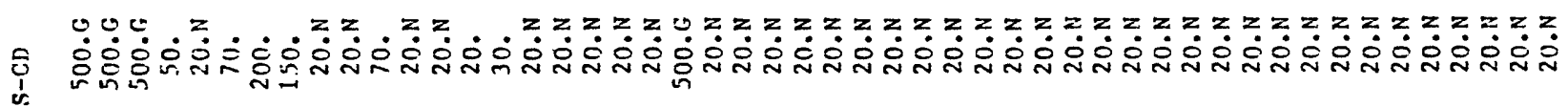

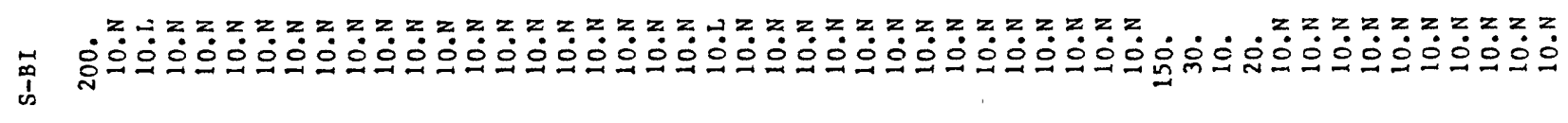

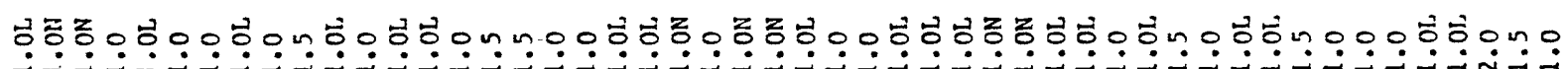

is

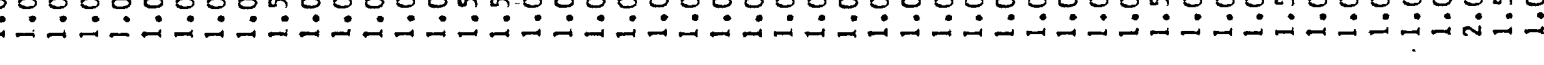

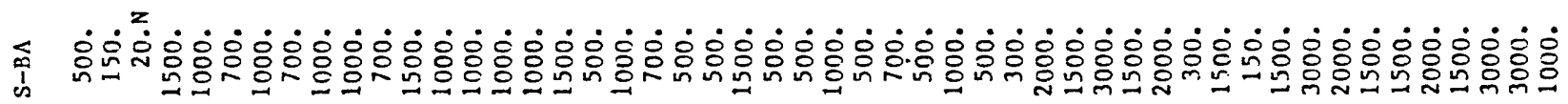

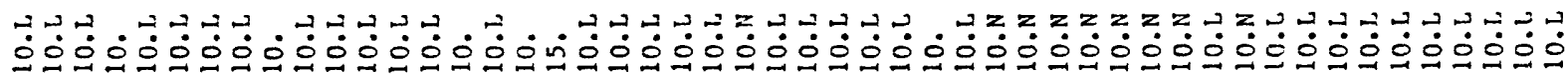


i

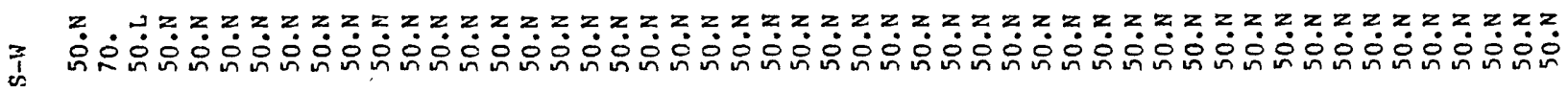

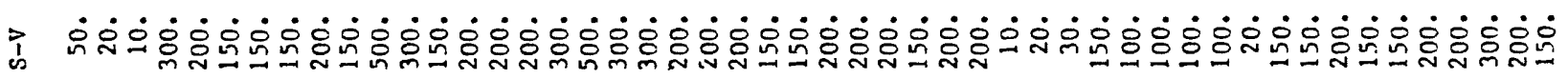

की

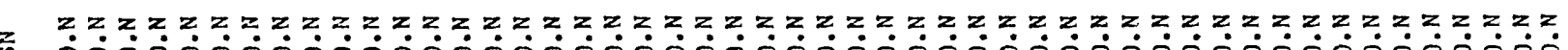
î

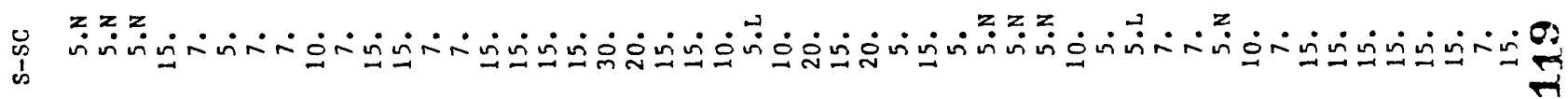

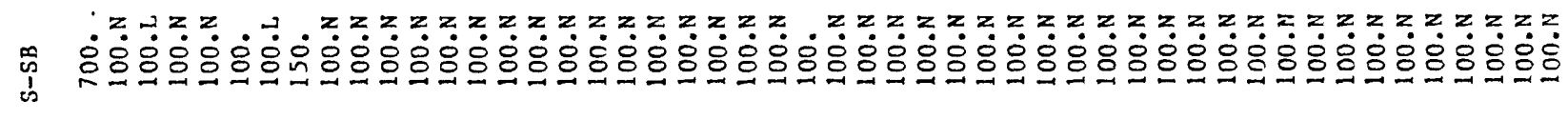

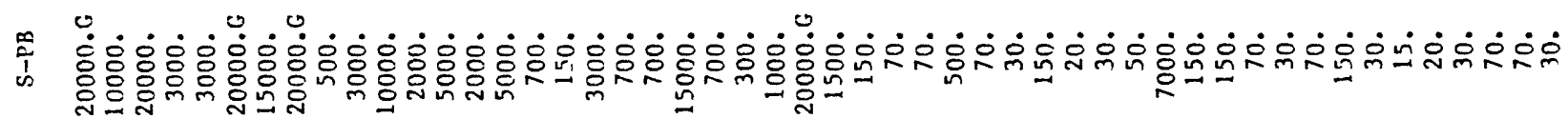

萑

苦

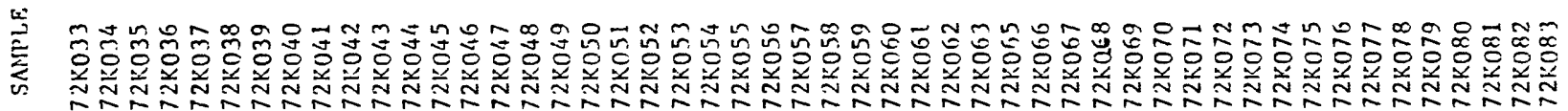




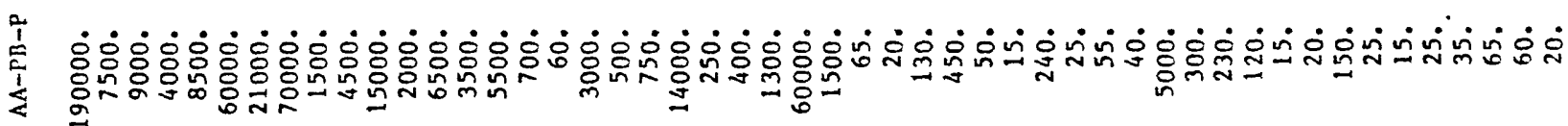

产

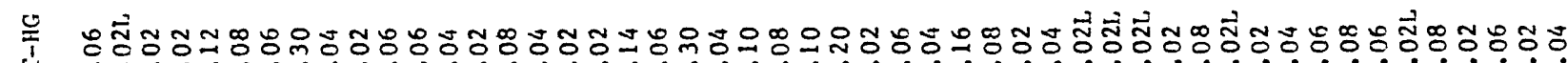

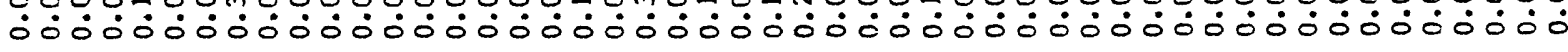

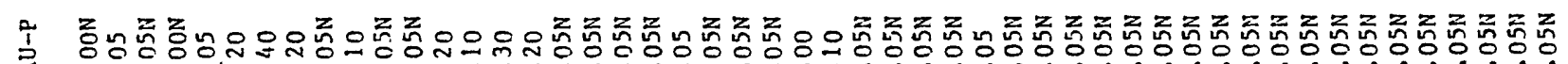

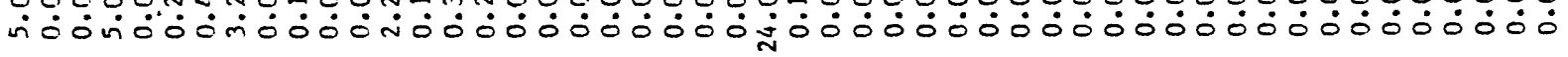

苑 z

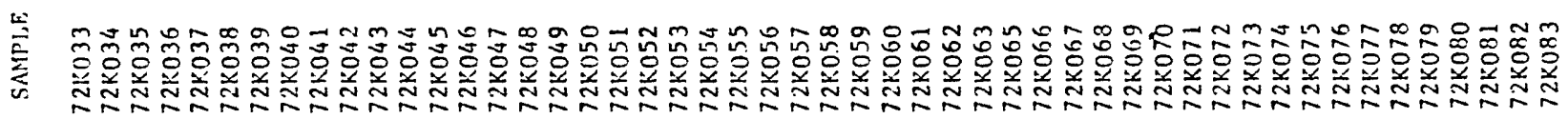


$z z z z z z z z z z z z z z z z z z z z z z z z z z z z z z z z z z z z z z z z z z z z z z z z z z$ s.

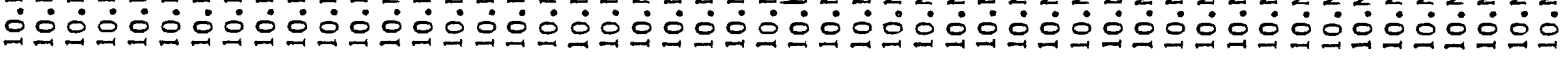

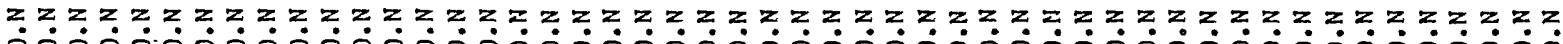

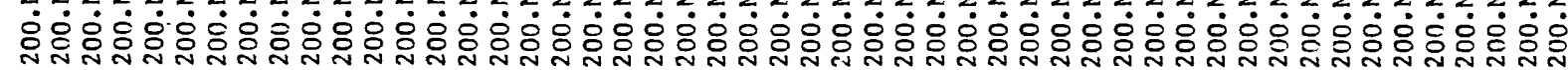

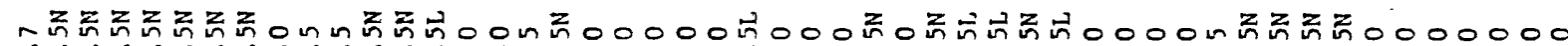

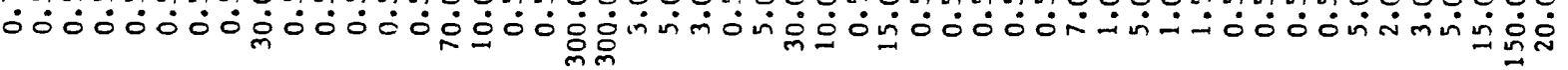

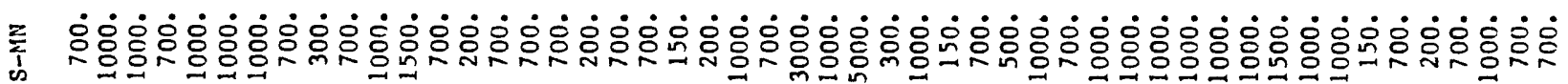

$\stackrel{0}{1}$

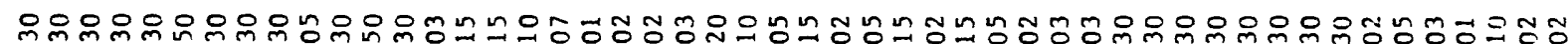
í $\dot{0} \dot{0} \dot{0} \dot{0} \dot{0} \dot{0} \dot{0} \dot{0} \dot{0} \dot{0} \dot{0} \dot{0} \dot{0} \dot{0} \dot{0} \dot{0} \dot{0} \dot{0} \dot{0} \dot{0} \dot{0} \dot{0} \dot{0} \dot{0} \dot{0} \dot{0} \dot{0} \dot{0} \dot{0} \dot{0} \dot{0} \dot{0} \dot{0} \dot{0} \dot{0} \dot{0}$

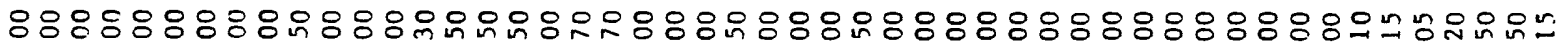

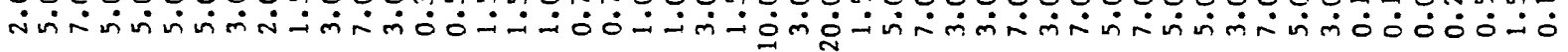

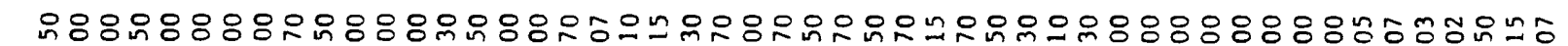

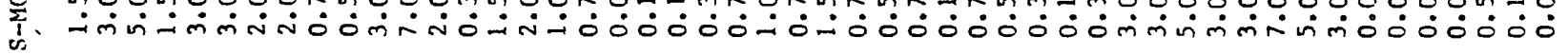

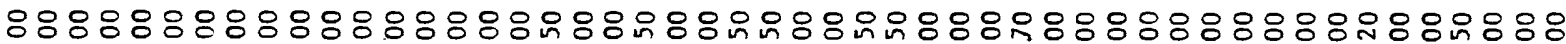

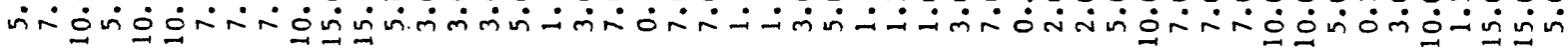

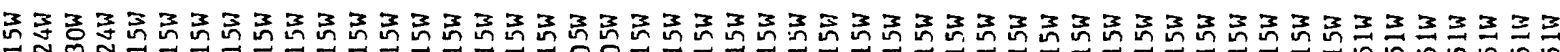

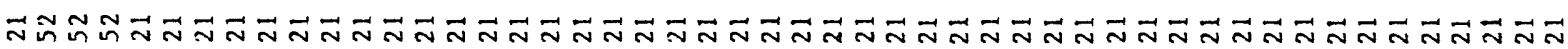

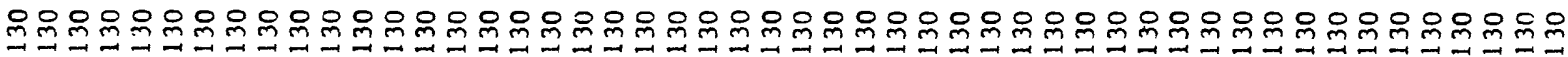

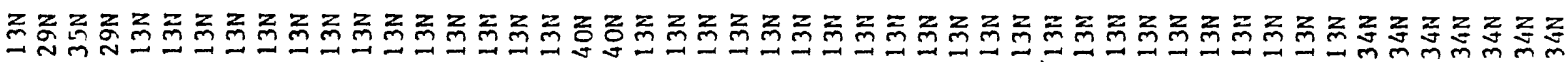

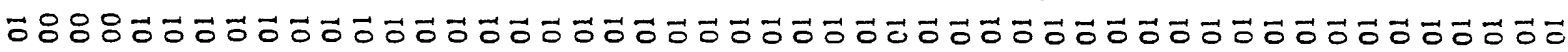

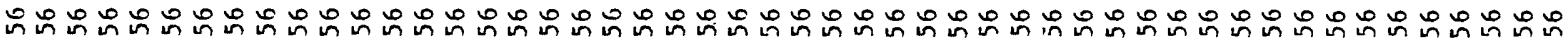




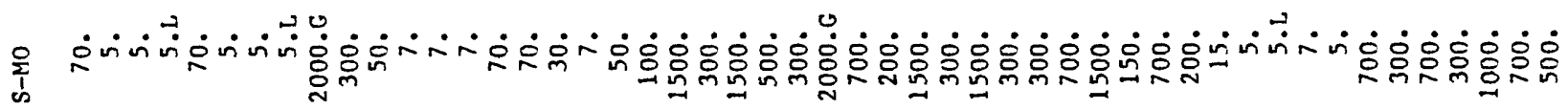

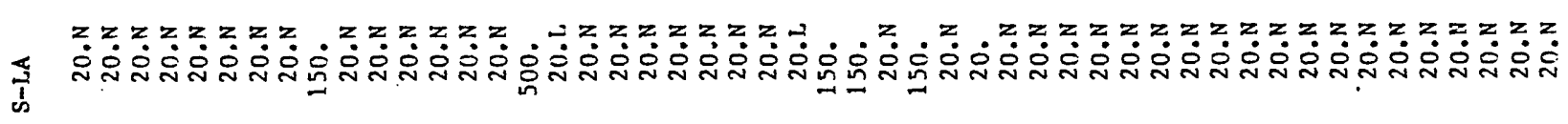
岁

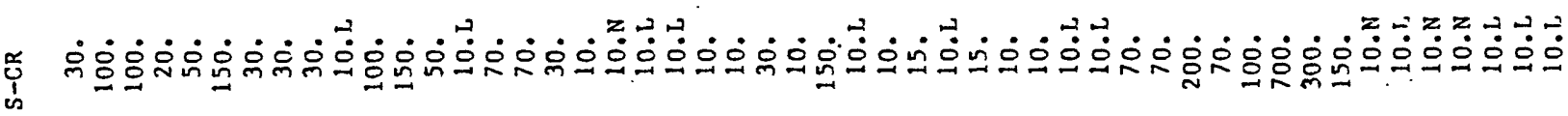

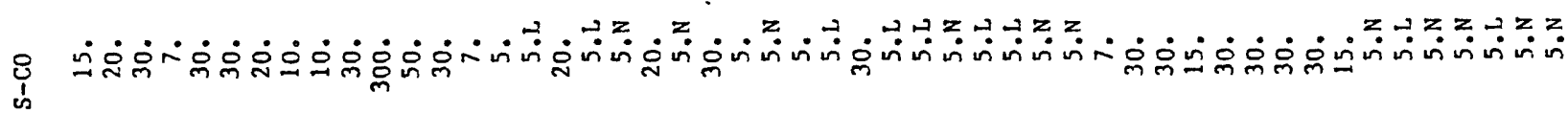

zzzzzzzzzzzzzzzzzzzzzzzzzzzzzzzzzzzzzzzzzzzzzzzzzz î́

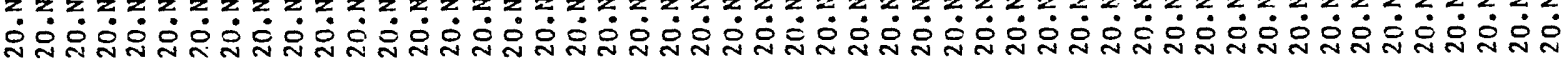

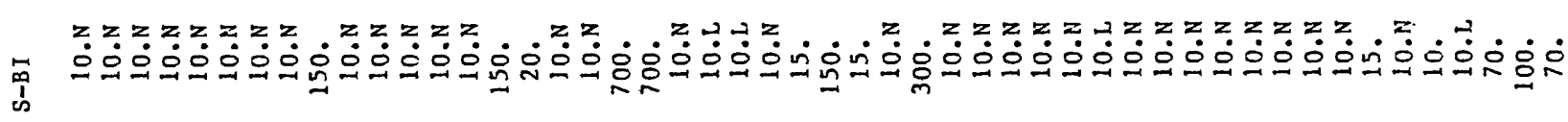

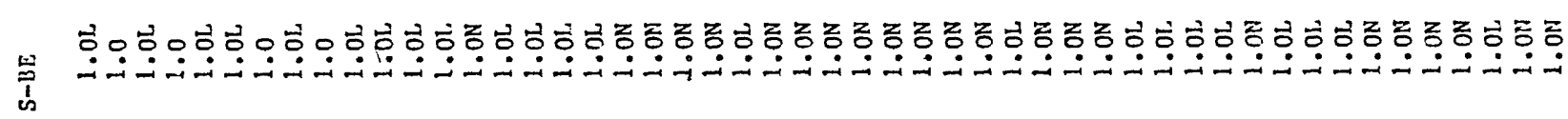

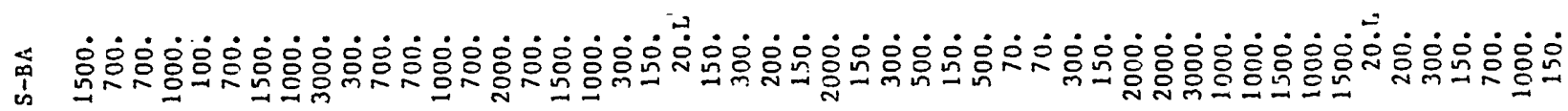

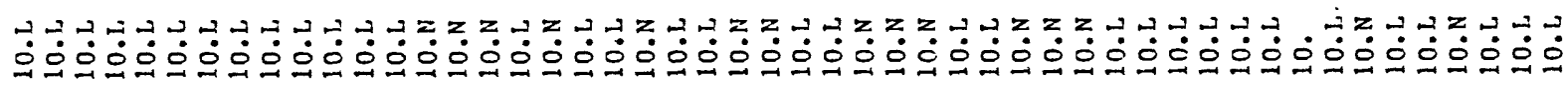
i⿱ 
म

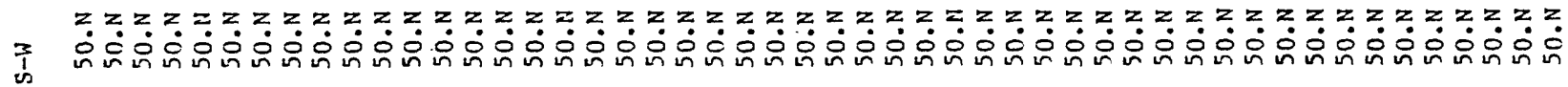

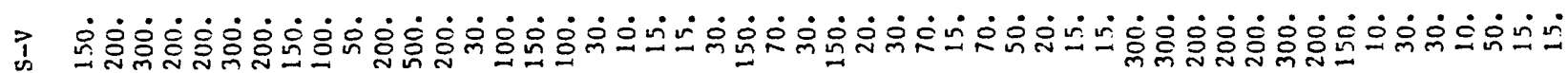

$z z z z z z z z z z z z z z z z z z z z z z z z z$ zzzzzzzzzzzzzzzzzzzzzzzz إ

u

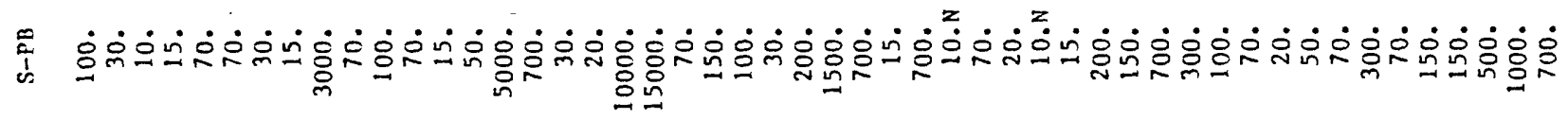

z

篎

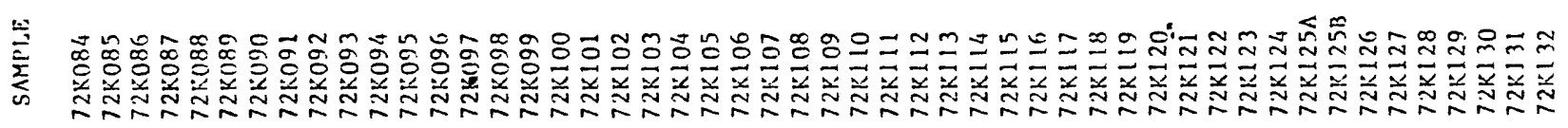




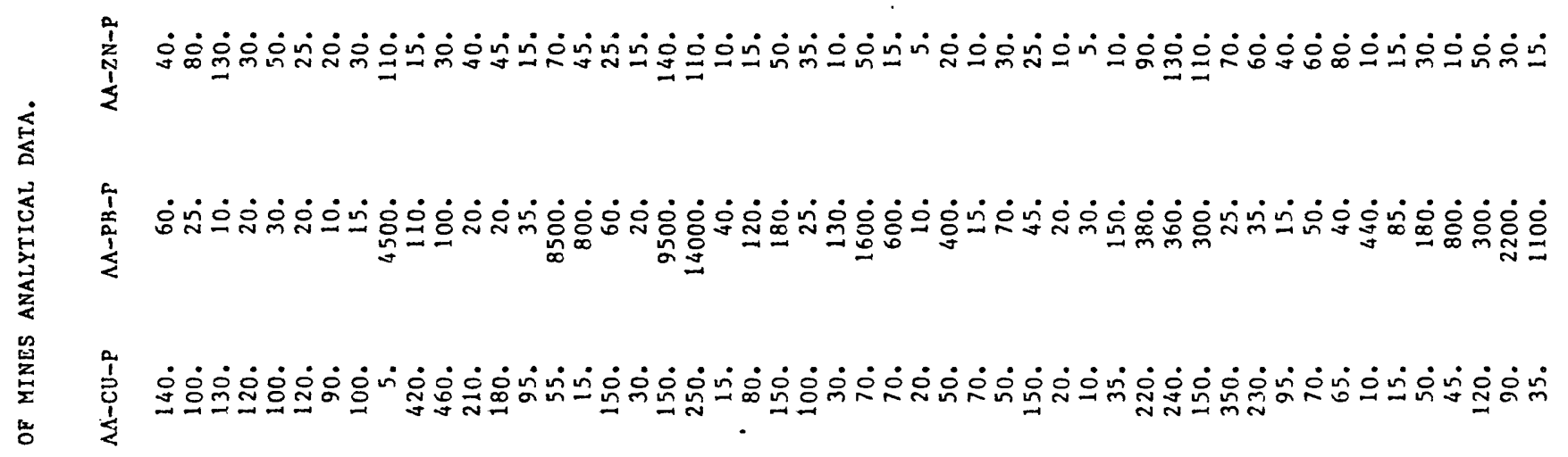

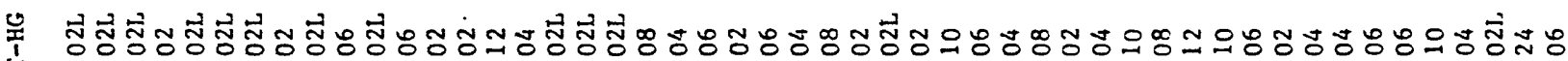

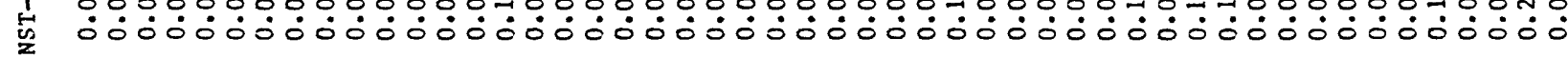

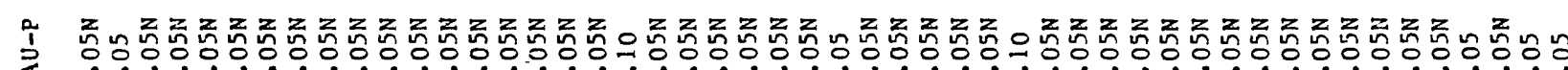

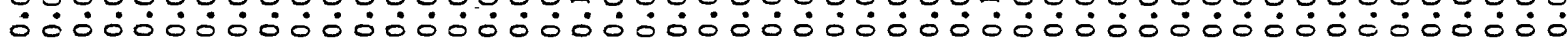

节

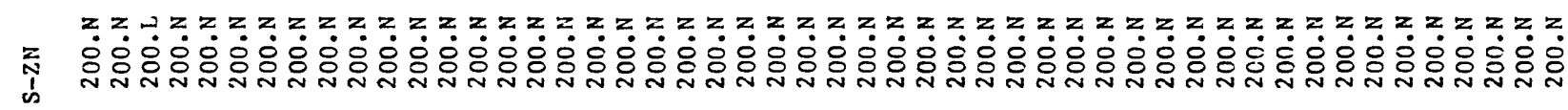




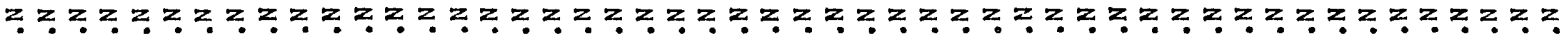

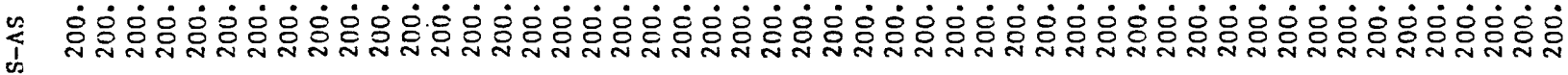

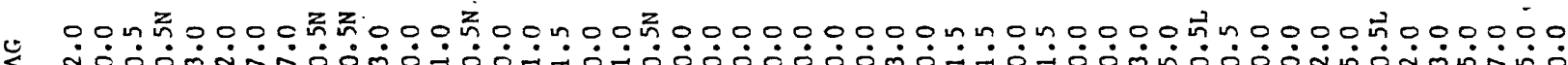
U

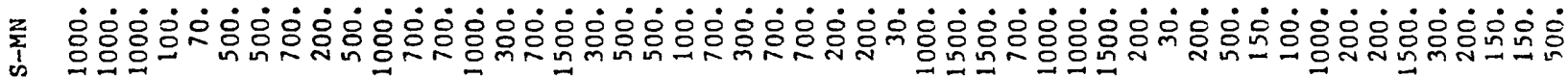

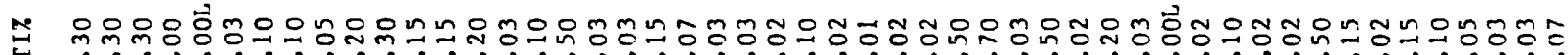
I

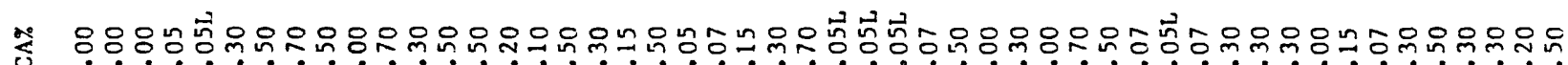
Í

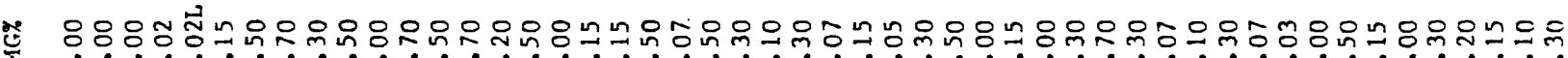
幽

운

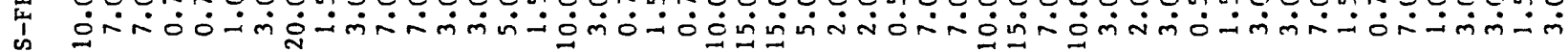

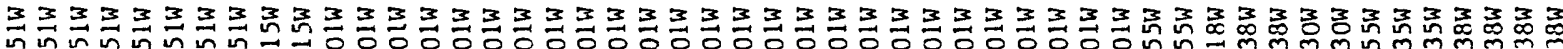

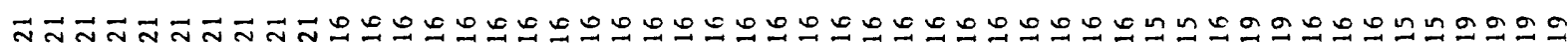

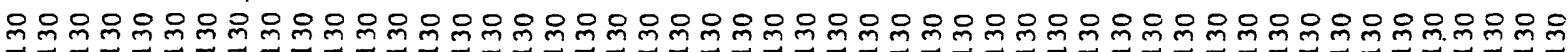

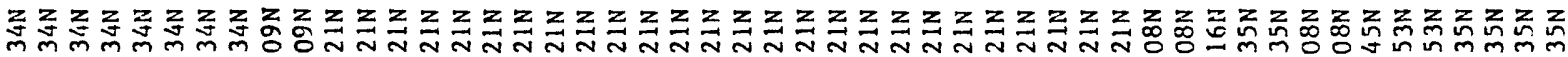

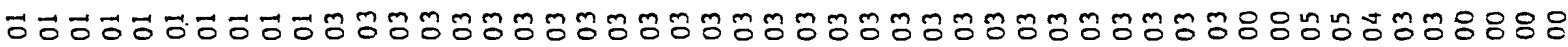

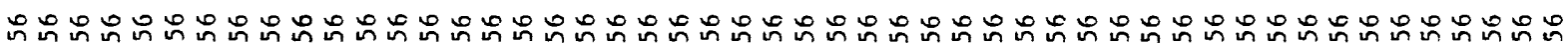

产 v (l) 


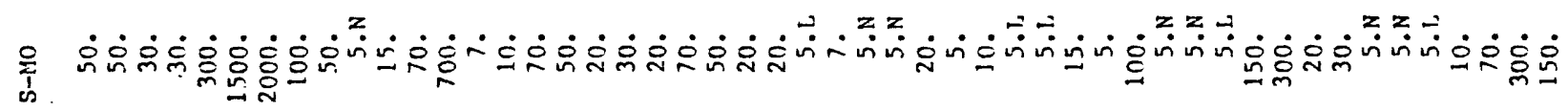

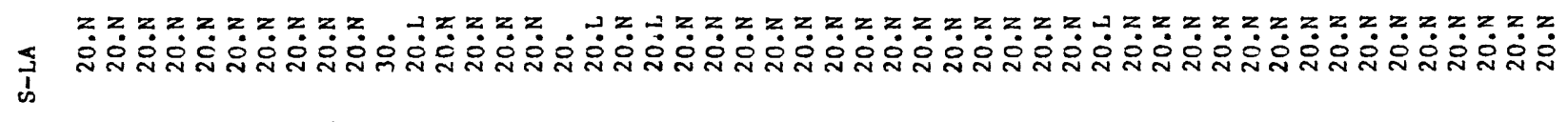
己 颌

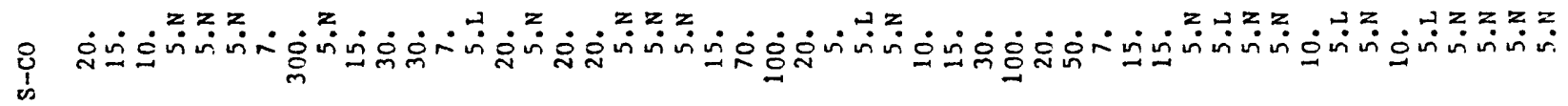

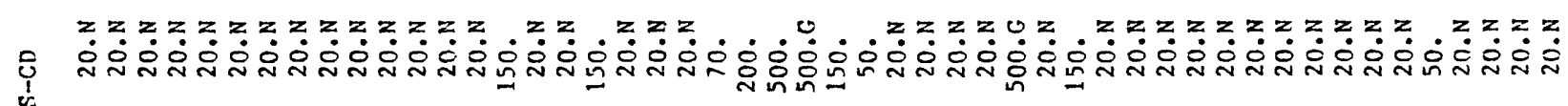
花 品

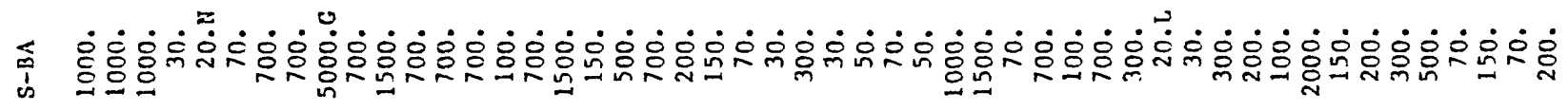
品 


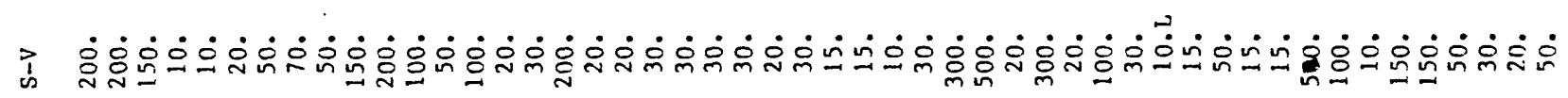

䧄

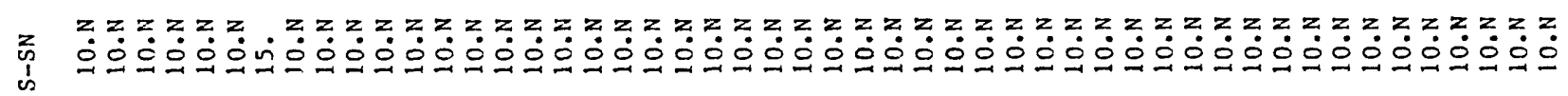

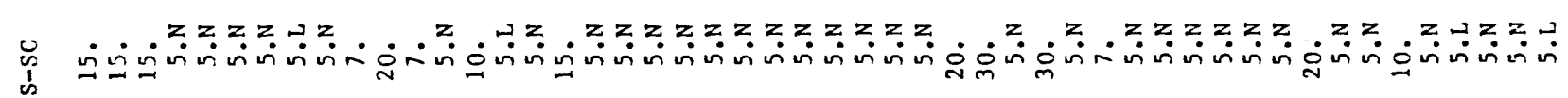

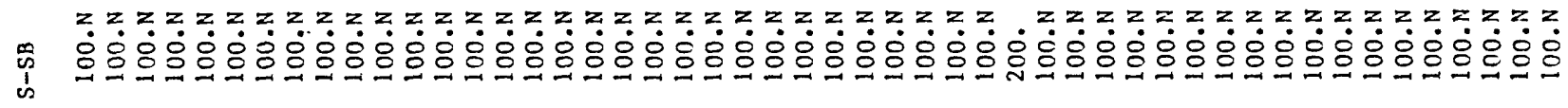

范

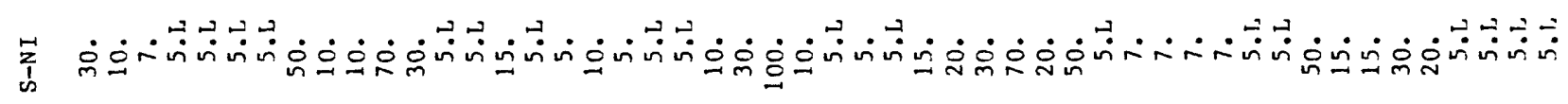

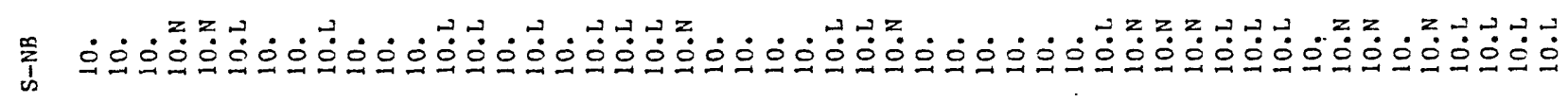

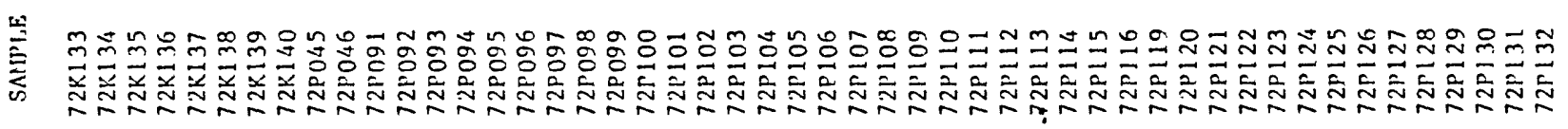




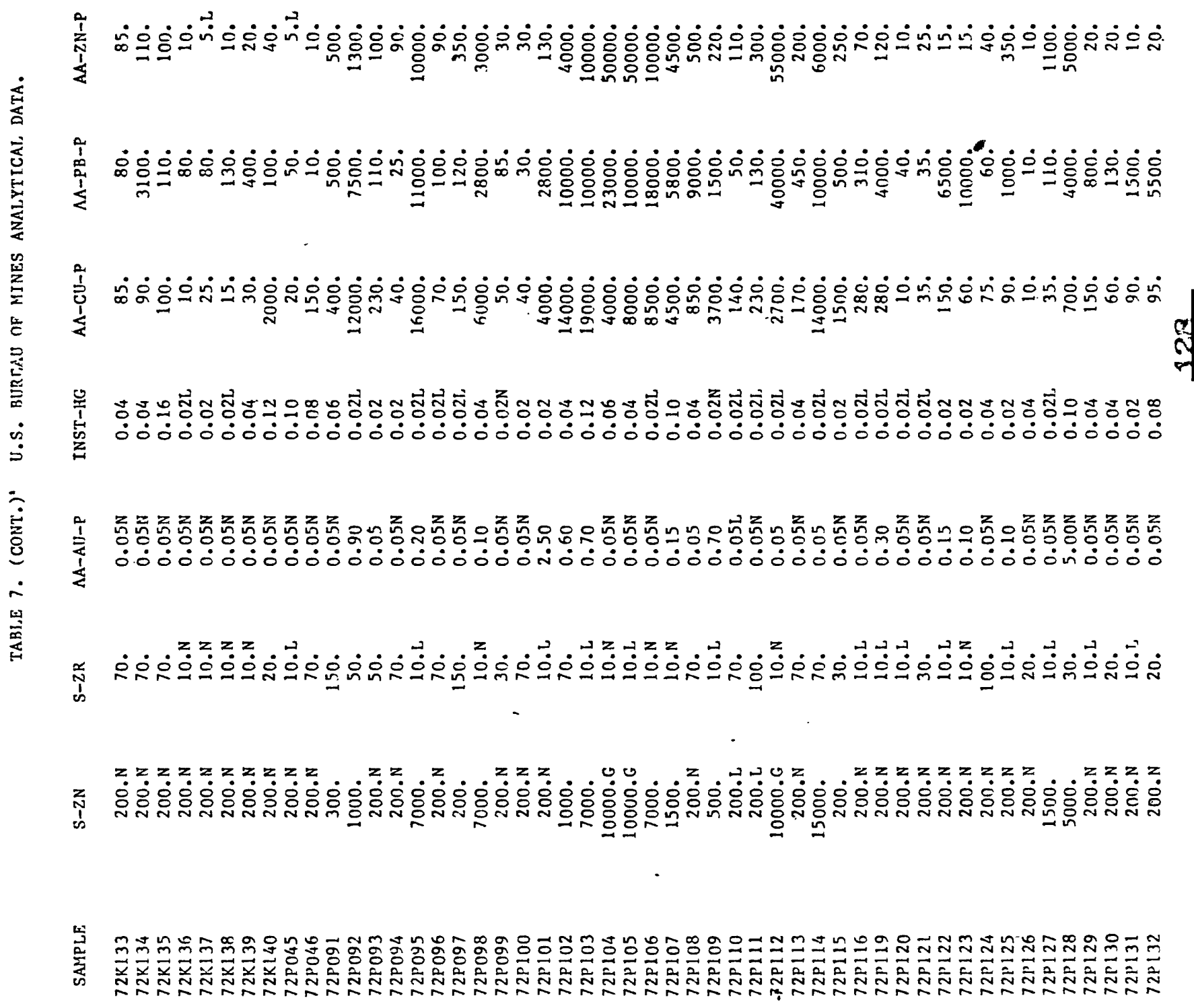




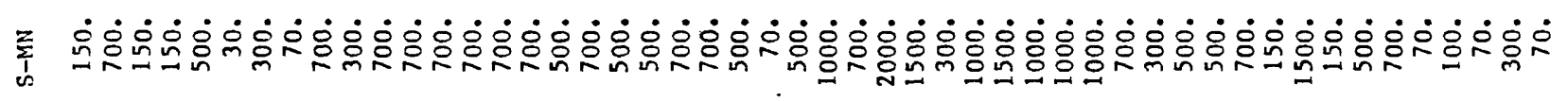

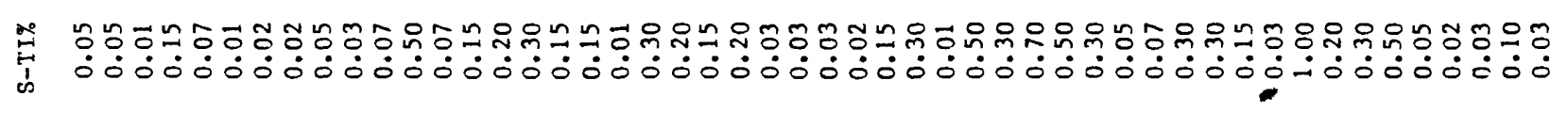

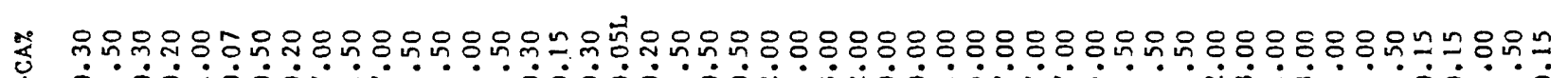
í

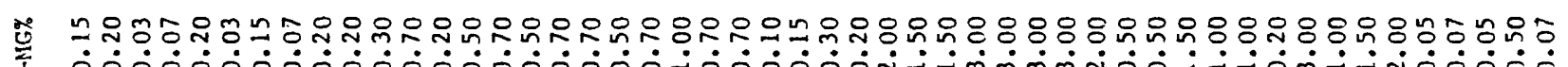
向

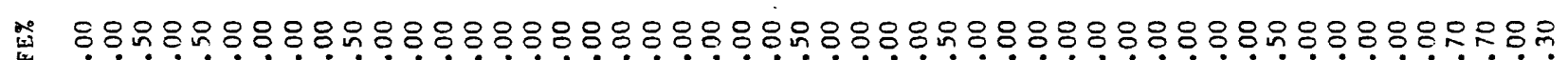

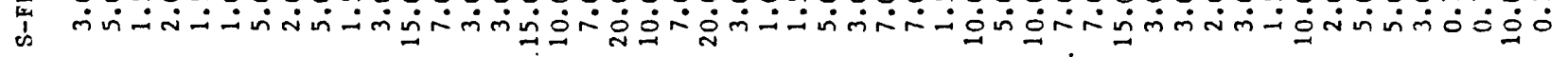

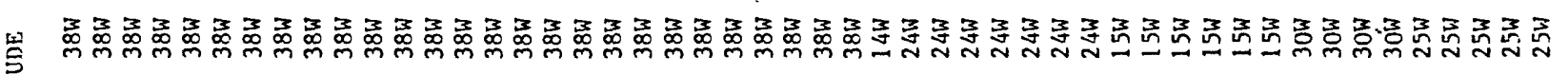

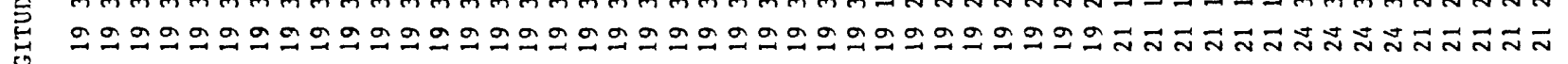

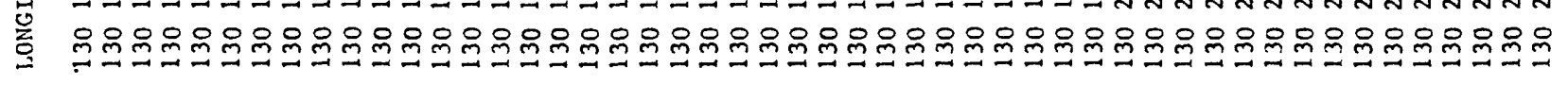

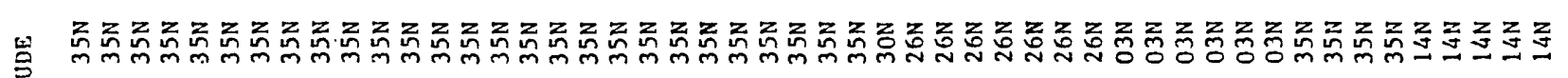

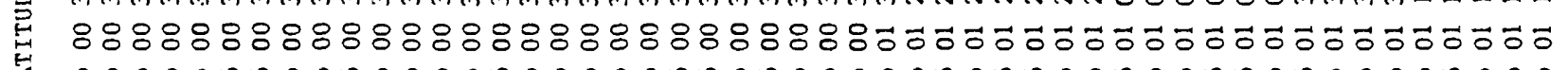

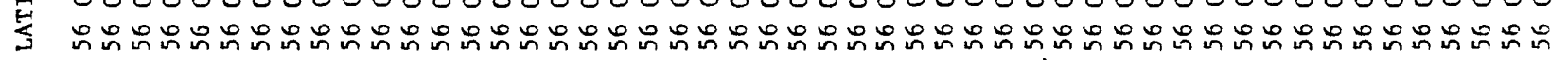

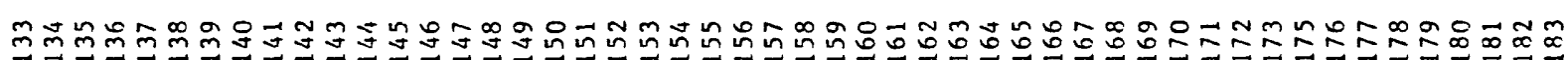

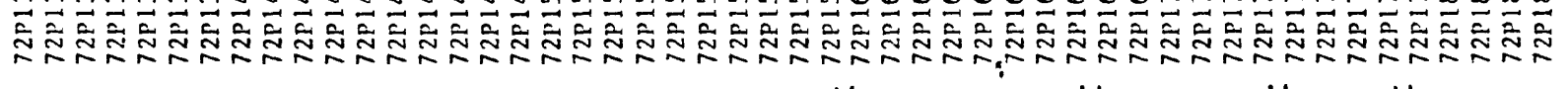




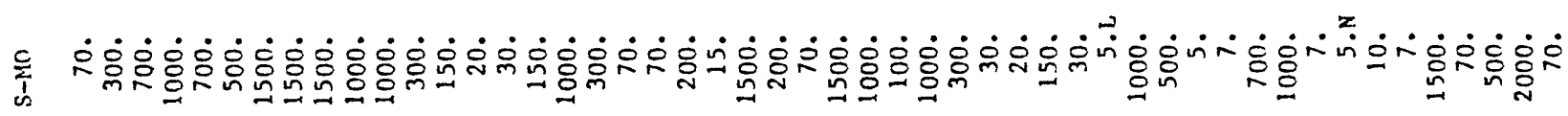

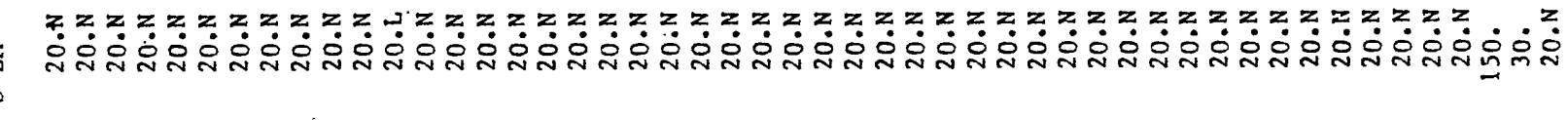
己 草

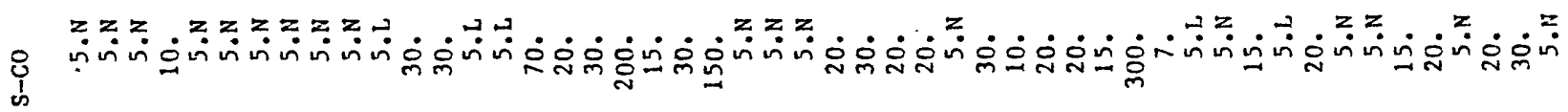

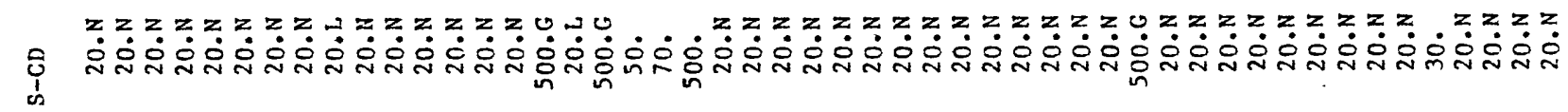

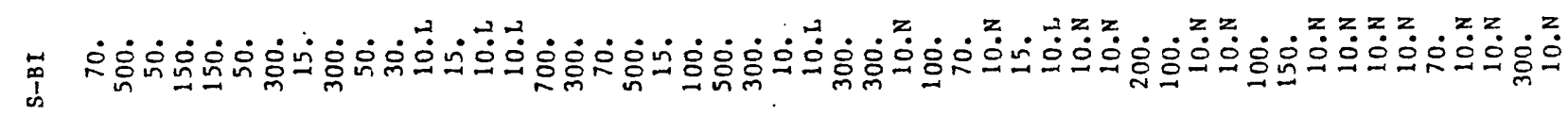
蓆 苑

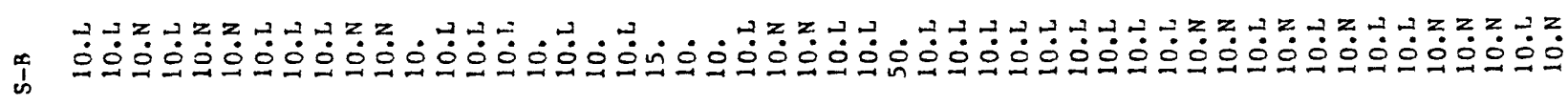

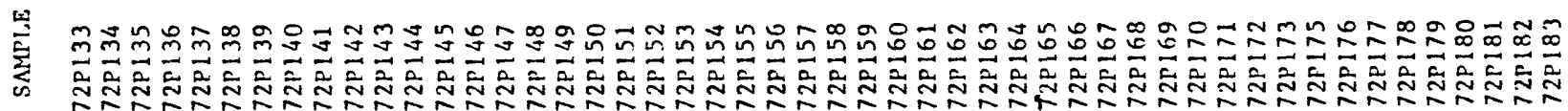


$\frac{N}{\frac{N}{n}}$

荌

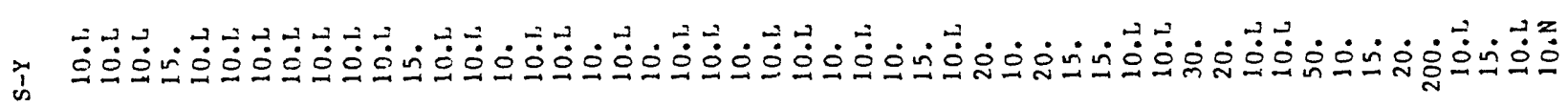

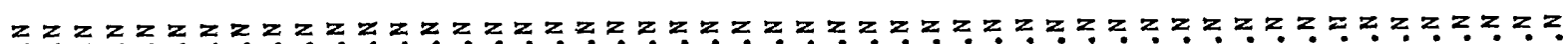
ơn

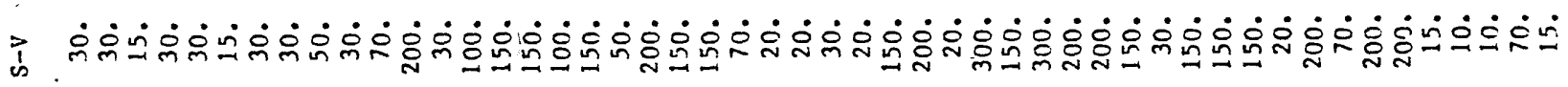

\%

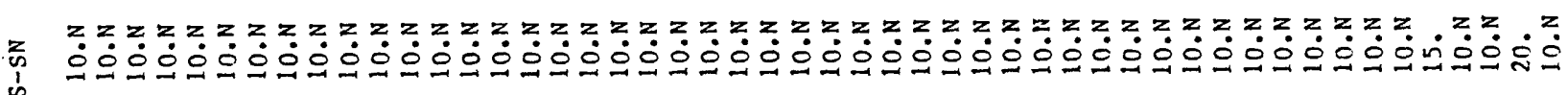

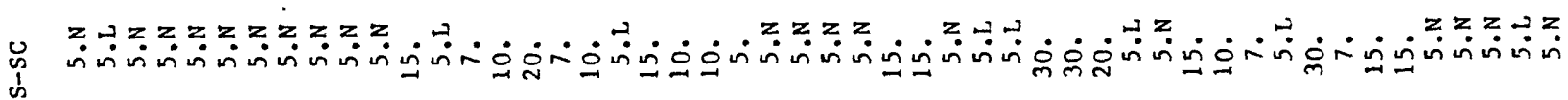

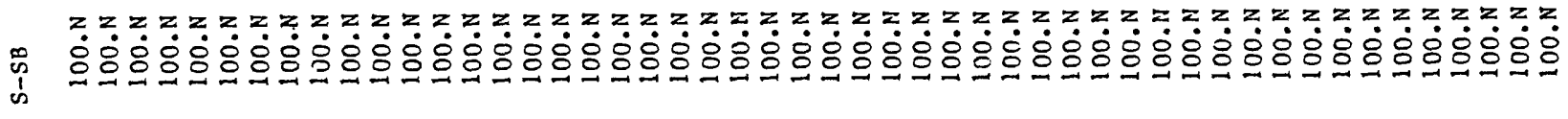

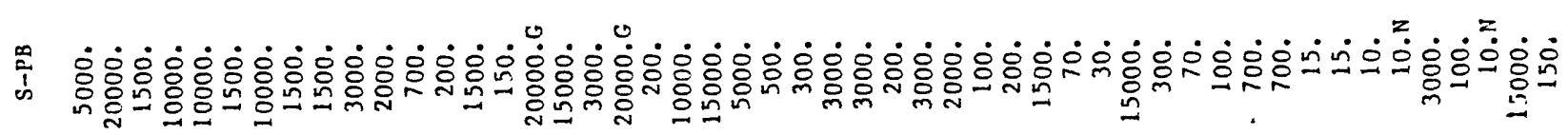

苛

管

象

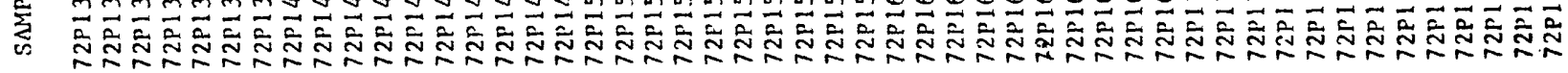


ํㅜㄴ 等

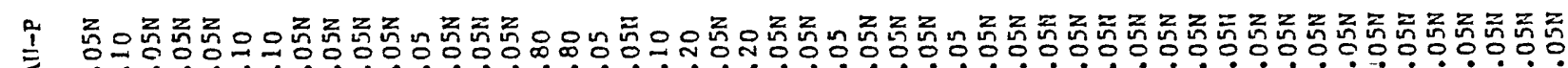
交

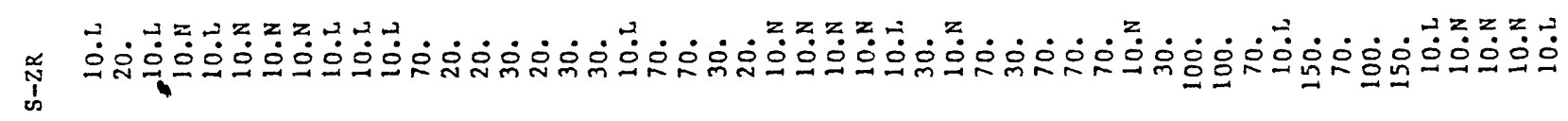

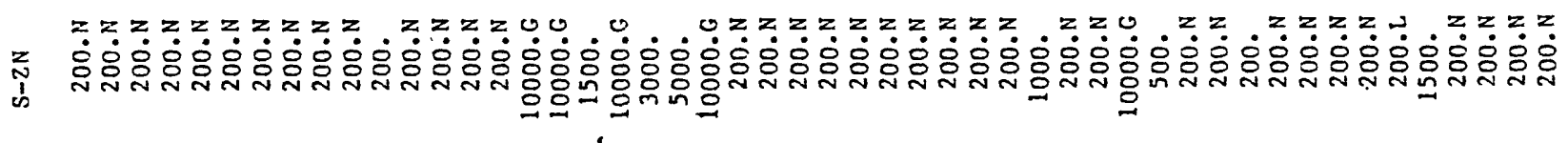

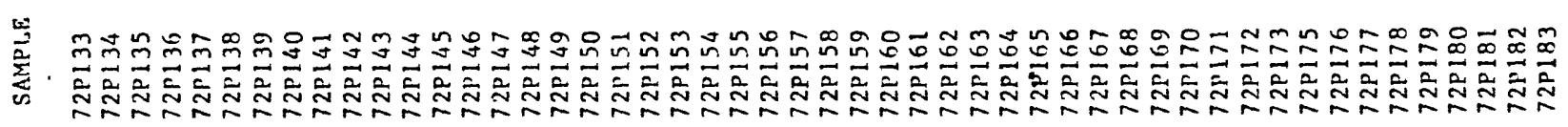




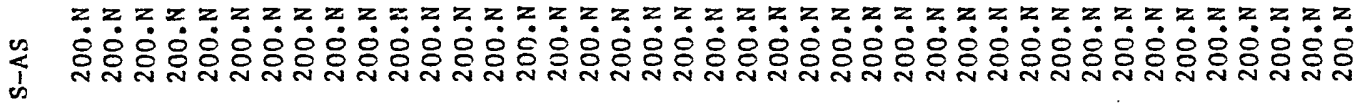

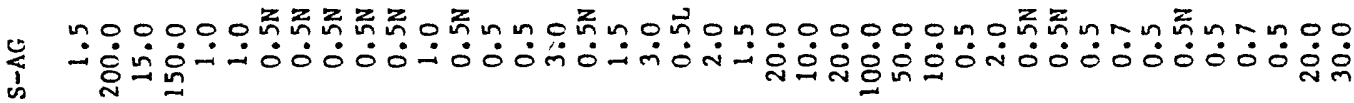

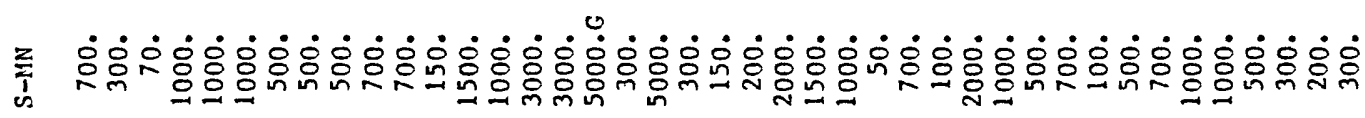

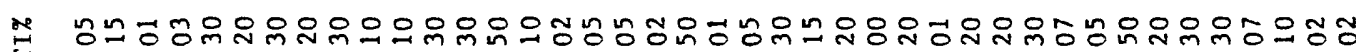

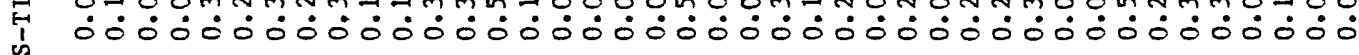

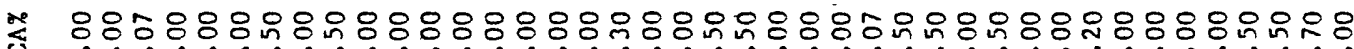

虫

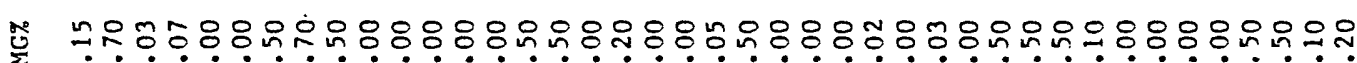

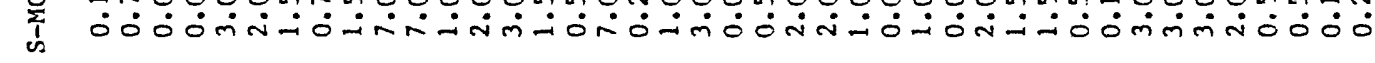

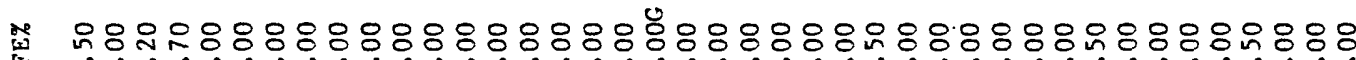

ஸ́

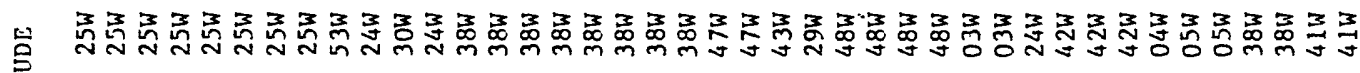

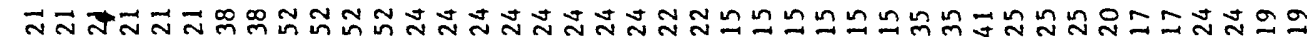

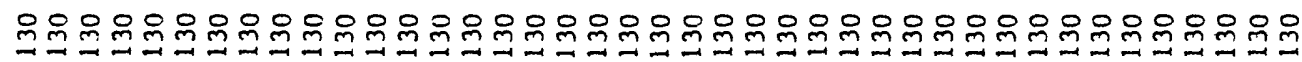

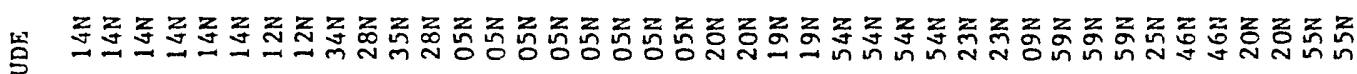
ธี่

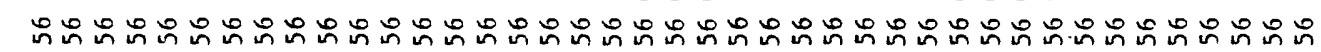

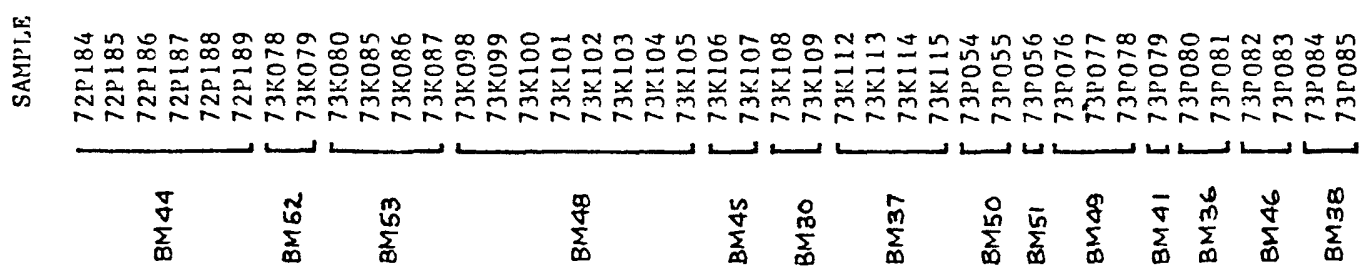




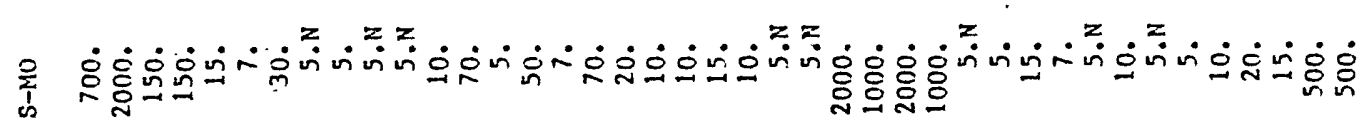
謇

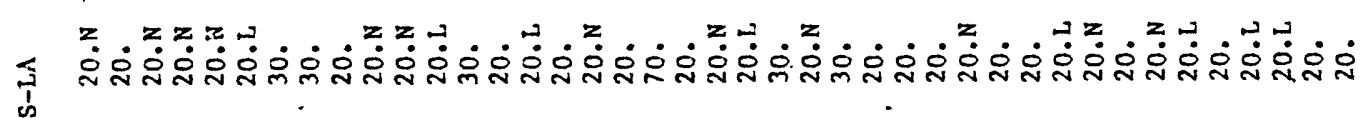

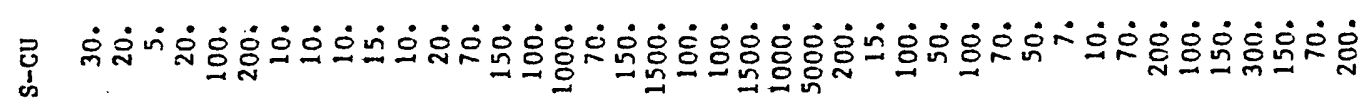
遂

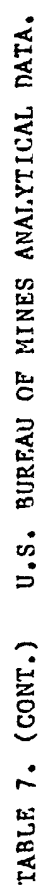

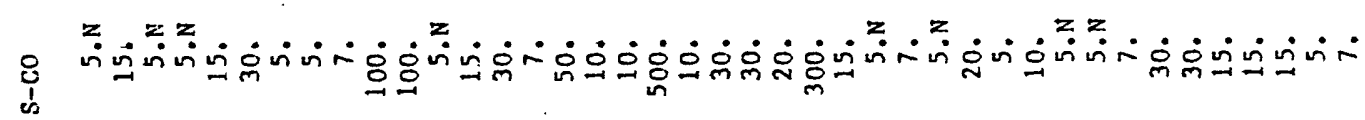

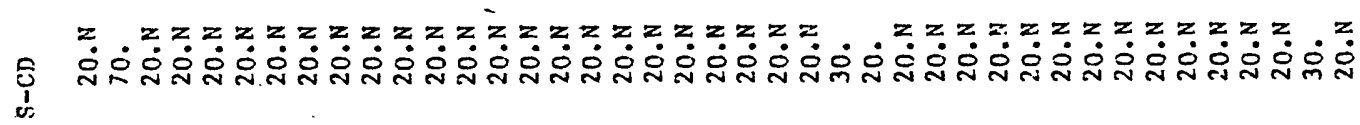

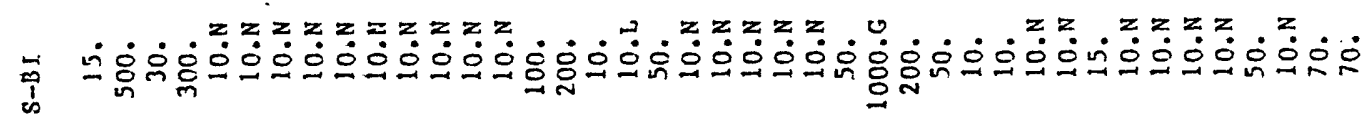

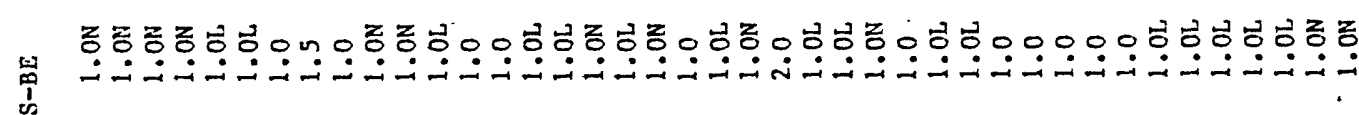

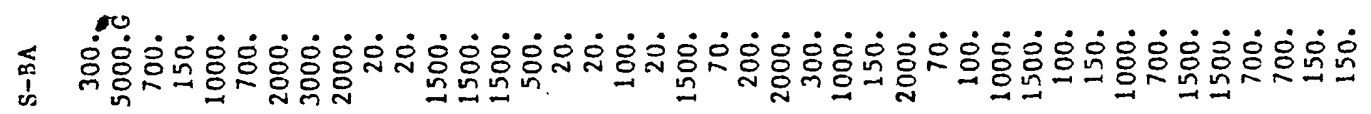

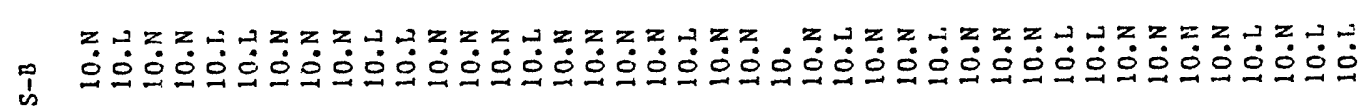

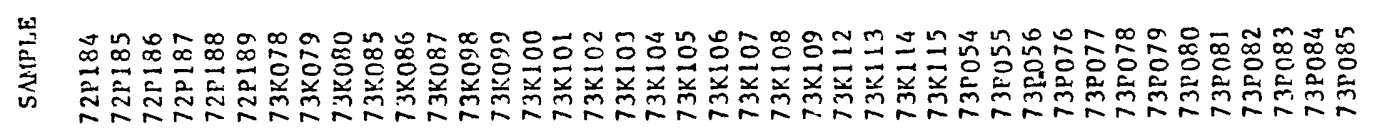




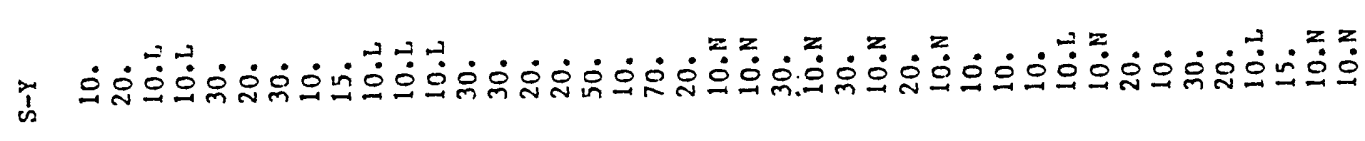

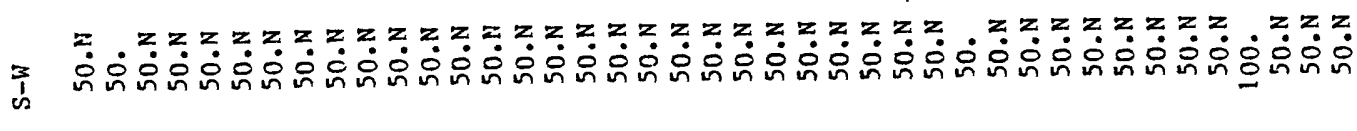

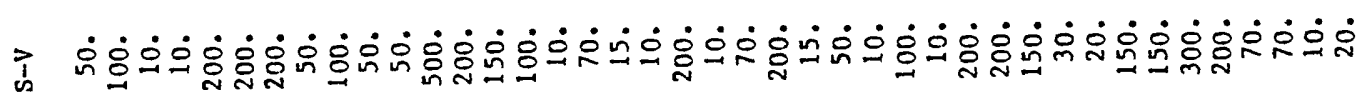

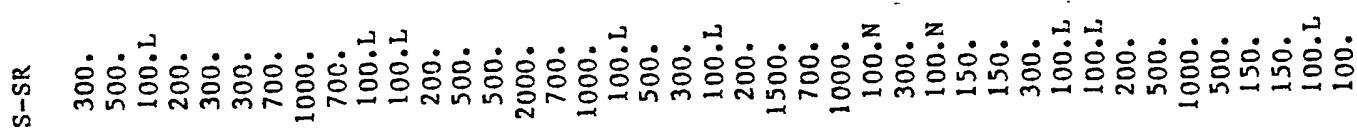

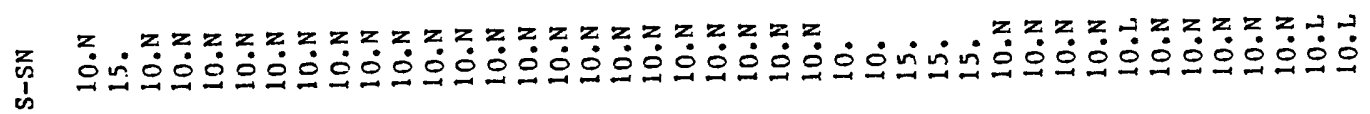

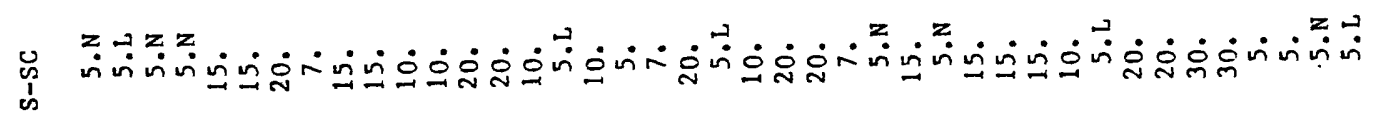


$\frac{2}{N}$

客

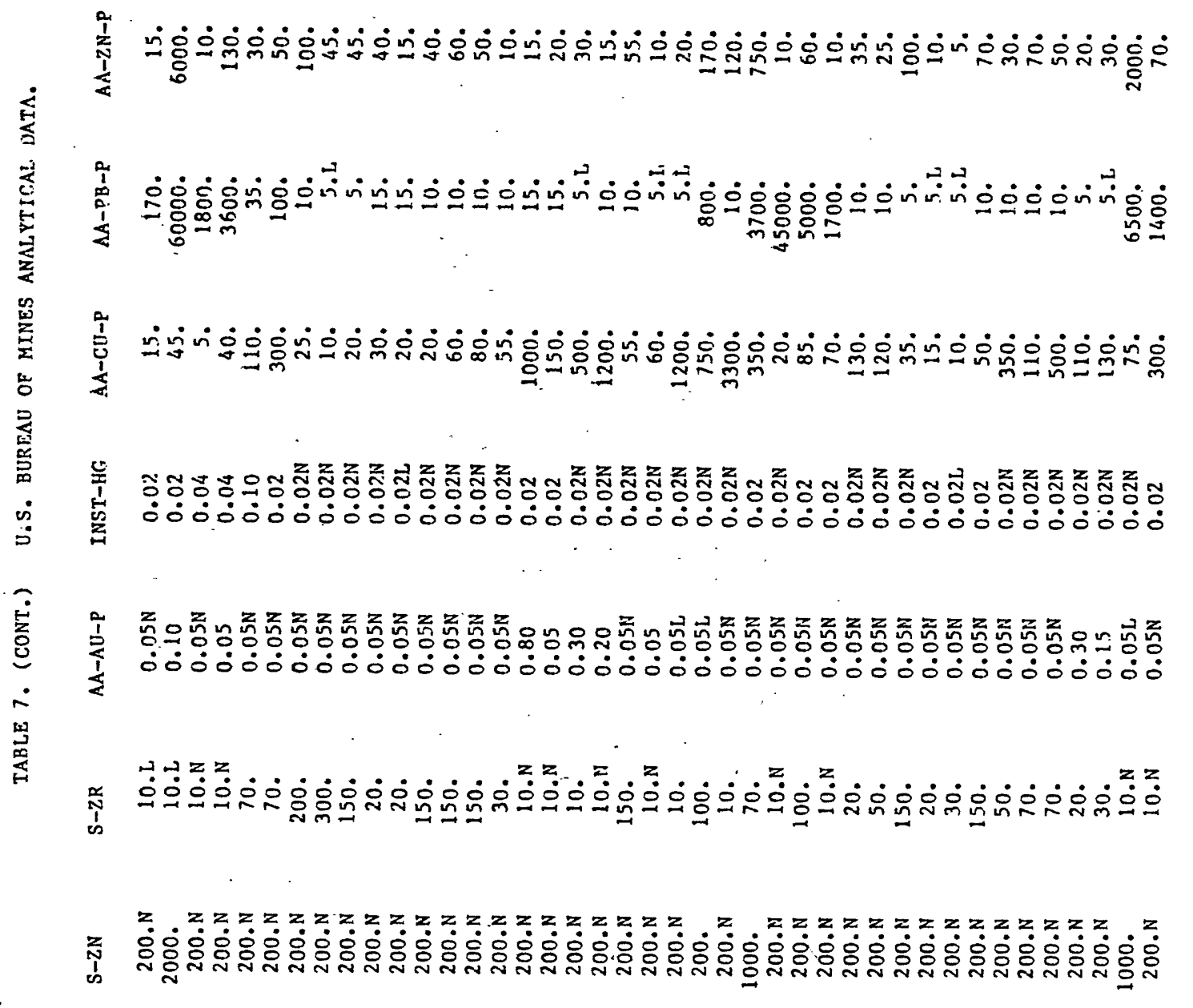

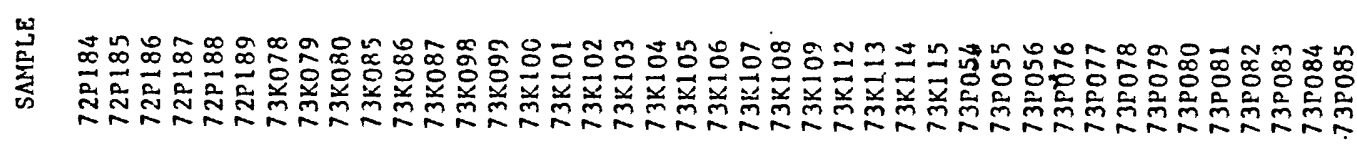

